
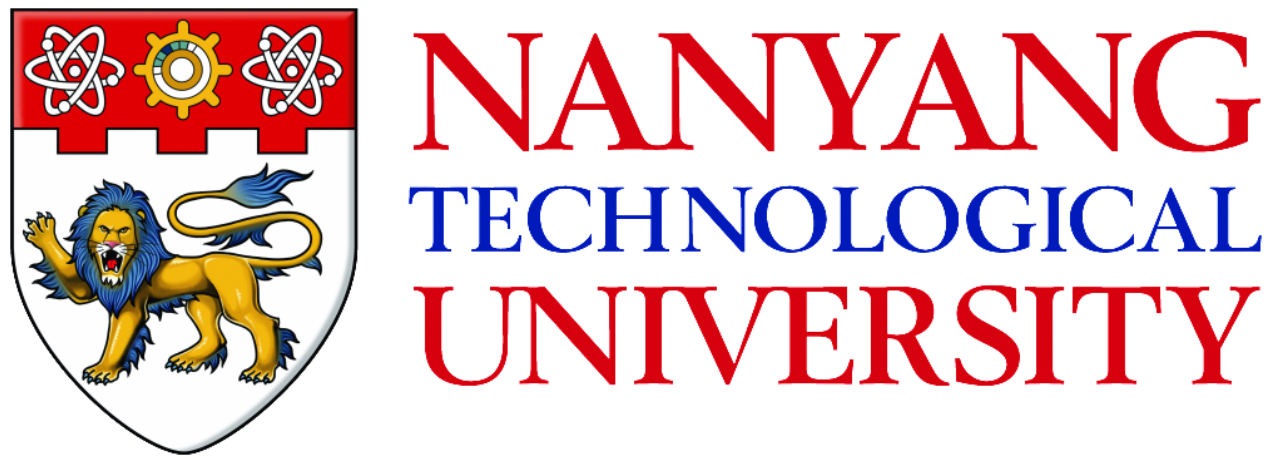

GRAPHENE QUANTUM DOTS (GQDS) AND THEIR DERIVATIVES FOR MULTIFARIOUS CATALYSIS AND ENVIRONMENTAL APPLICATIONS

\author{
ZENG ZHIPING \\ Interdisciplinary Graduate School
}

Nanyang Environment \& Water Research Institute @ NTU 


\section{GRAPHENE QUANTUM DOTS (GQDS) AND THEIR \\ DERIVATIVES FOR MULTIFARIOUS CATALYSIS AND \\ ENVIRONMENTAL APPLICATIONS}

ZENG ZHIPING

Interdisciplinary Graduate School

Nanyang Environment \& Water Research Institute

Singapore Membrane Technology Centre (SMTC)

A thesis submitted to the Nanyang Technological University in partial fulfillment of the requirement for the degree of

Doctor of Philosophy 


\section{Acknowledgement}

First of all, I would like to express my deepest respect and greatest appreciation to my supervisor Associate Professor Timothy Tan and co-supervisor Professor Wang Rong, for their excellent guidance, encouragement and support. They always try their best to answer any of my queries, and are also glad to provide experiences and instructions to me.

Secondly, I sincerely thank Dr. Xiao Fang-Xing, Dr. Yu Dingshan, Dr. He Ziming, Dr Liu Jin, Dr Zhang Yan, Yu Zhongzhen and Jodie Tan Zu Yi for their patient guidance and kindness. The professional knowledge, scientific writing skills and creative research methodologies learned from them are most valuable to me.

I am also grateful to Professor Thuc-Quyen Nguyen and Dr Hung Phan from University of California Santa Barbara, Dibakar Bhattacharyya from University of Kentucky for the supportive collaborations and valuable suggestions they provided. Their instructions greatly help me develop a good understanding in material science and technology.

I would like to thank Singapore Membrane Technology Centre (SMTC) and Nanyang Environment \& Water Research Institute (NEWRI) for providing me the opportunity and scholarship to pursue my $\mathrm{PhD}$ degree.

Last but not least, I wish to express my heartfelt gratitude to my parents, Zeng Biaohong and Liu Shimei, and my beloved wife, Chen Shufen, for their everlasting love and support throughout my life. 


\section{Publications}

1. Zeng Z.; Xiao F.-X.; Gui X.; Wang R.; Liu B.; Tan T. Y. T., Layer-by-layer assembly of nitrogen-doped graphene quantum dots monolayer decorated onedimensional semiconductor nanoarchitectures for solar-driven water splitting. Journal Materials Chemistry A, 2016, 4, 16383-16393.

2. Zeng Z.; Yu D.; He Z.; Liu J.; Xiao F.-X.; Zhang Y.; Wang R.; Dibakar B.; Tan T. Y. T., Graphene Oxide Quantum Dots Covalently Functionalized PVDF Membrane with Significantly-Enhanced Bactericidal and Antibiofouling Performances. Scientific Report, 2016, 6, 20142.

3. Zeng Z.; Xiao F.-X.; Phan H.; Chen S. F.; Wang R.; Nguyen T.-Q.; Tan T. Y. T., Unraveling Cooperative Synergy of Carbon Nanomaterials and Metal Nanocrystals in Multilayered Architectures towards Multifarious Catalysis. 2017, In submission.

4. Zeng Z.; Xiao F.-X.; Tan T. Y. T.; Graphene quantum dots (GQDs) and their derivatives for sustainable energy and environmental applications, 2017, In submission.

5. Zhang Y.; Yu Z.; Li J.; Ao Y.; Xue J.; Zeng Z.; Yang X.; Tan T. T. Y., Ultrasmall-super bright neodymium-upconversion nanoparticles via energy migration manipulation and lattice modification: $808 \mathrm{~nm}$-activated drug release. ACS Nano, 2017, 11, 2846-2857. 


\section{Table of contents}

Acknowledgement .................................................................................................................

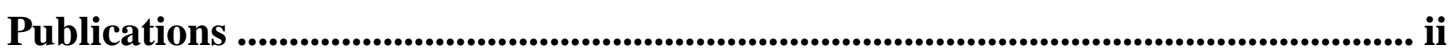

Table of contents ....................................................................................................... ii

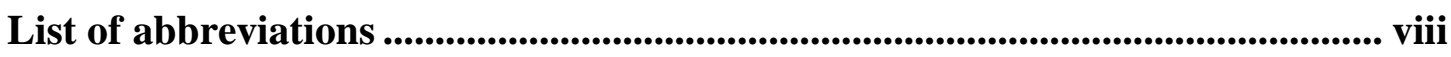

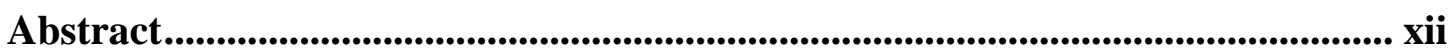

Chapter 1 Introduction.........................................................................................................1

1.1 Background and significance.................................................................1

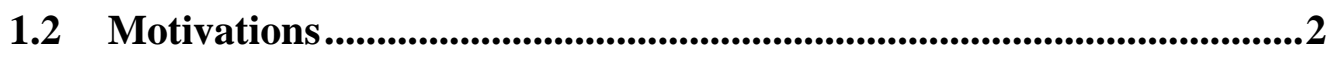

1.3 Objectives .........................................................................................................4

1.4 Organization of the dissertation ..................................................6

Chapter 2 Literature review ...................................................................................................9

2.1 Fabrication methods of GQDs ...............................................................9

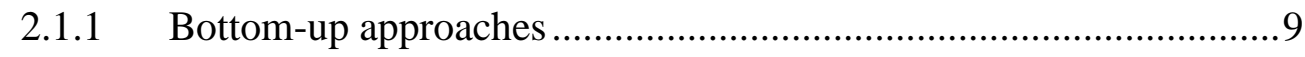

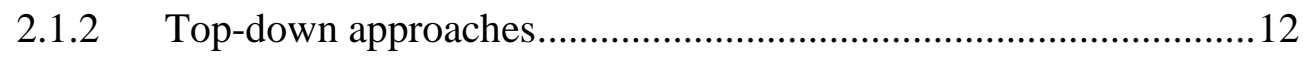

2.2 GQDs-based functionalized materials for multifarious catalysis performance

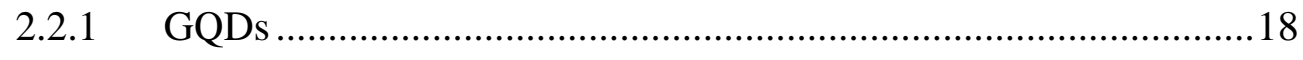

2.2.2 GQDs-based 0 D materials ...................................................23

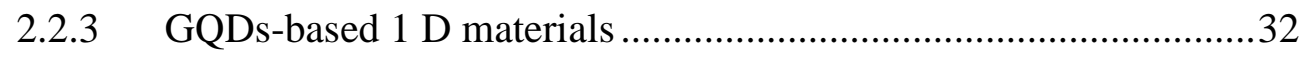

2.2.4 GQDs-based 2 D materials .................................................... 35

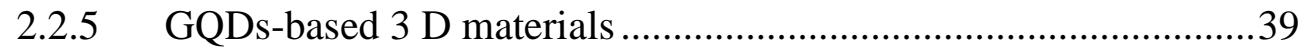


Chapter 3 Unraveling Cooperative Synergy of Carbon Nanomaterials and Metal Nanocrystals in Multilayered Architectures towards Multifarious Catalysis......45

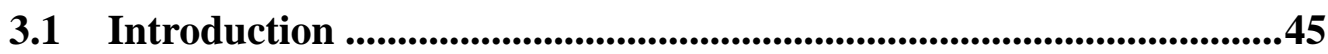

3.2 Experimental ...............................................................................47

3.2.1 Preparation of graphene oxide quantum dots (GQDs) .................47

3.2.2 Synthesis of negatively charged citrate-stabilized metal nanocrystals............................................48

3.2.3 LbL assembly of (M/GQDs)n (M=Au, Ag, Pt $)$ multilayer composite thin films

3.2.4 Materials Characterizations .......................................................50

3.2.5 Selective catalytic reduction of aromatic nitro compounds............51

3.2.6 Electrochemical methanol oxidation ........................................51

3.2.7 Photoelectrochemical (PEC) Measurement................................52

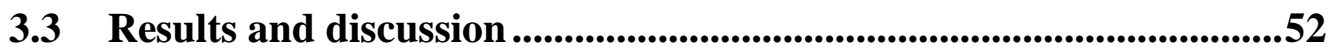

3.3.1 Characterization of GQDs, metal nanocrystals and (M/GQDs) $(\mathrm{M}=\mathrm{Au}, \mathrm{Ag}, \mathrm{Pt})$ nanostructures

3.3.2 Selective catalytic reaction performance of $(\mathrm{M} / \mathrm{GQDs})_{\mathrm{n}}(\mathrm{M}=\mathrm{Au}, \mathrm{Ag}$, Pt) hybrid nanostructures .66

3.3.3 Electrochemical catalytic performance of (M/GQDs)n ( $\mathrm{M}=\mathrm{Au}, \mathrm{Ag}$, Pt) hybrid nanostructures .71

3.3.4 Photoelectrochemical catalytic performance of $(\mathrm{M} / \mathrm{GQDs})_{\mathrm{n}}(\mathrm{M}=\mathrm{Au}$, $\mathrm{Ag}, \mathrm{Pt}$ ) hybrid nanostructures .76 
3.3.5 Selective catalytic reduction, electrochemical and photoelectrochemical catalytic mechanisms .............................79

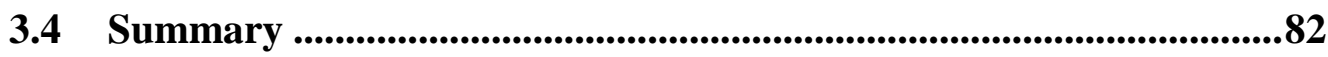

Chapter 4 Layer-by-Layer of nitrogen-doped graphene quantum dots monolayer decorated one-dimensional semiconductor nanoarchitectures for solar-driven

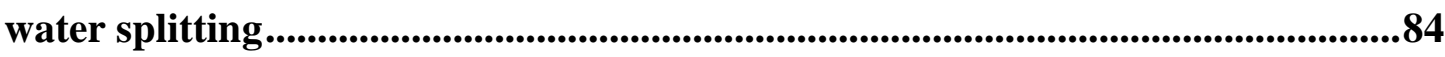

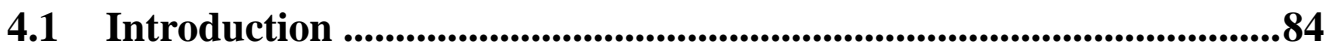

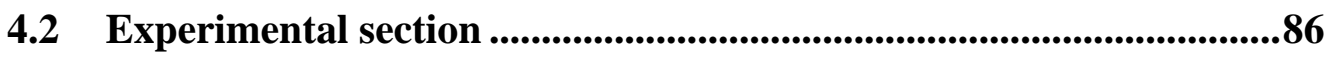

4.2.1 Fabrication of nitrogen-doped GQDs, ZnO NWs, TiO2 NRs ........86

4.2.2 LbL assembly of N-GQDs/ZnO NWs (N-GQDs/TiO2 NRs) and GQDs/ZnO NWs (GQDs/TiO2 NRs) heterostructures .................88

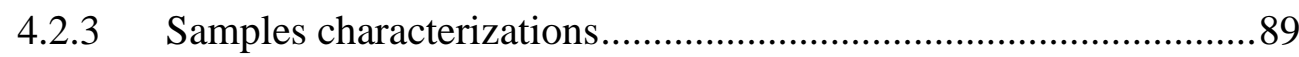

4.2.4 PEC water splitting performances measurements .......................89

4.3 Results and discussion ...................................................................90

4.3.1 Synthesis and characterization of N-GQDs .................................90

4.3.2 LbL assembly of N-GQDs/ZnO NWs heterostructures ................95

4.3.3 Photoelectrochemical water splitting performances .....................103

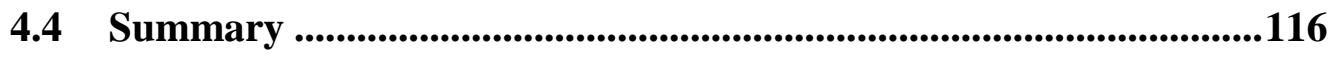

Chapter 5 Graphene Oxide Quantum Dots Covalently Functionalized PVDF Membrane with Significantly-Enhanced Bactericidal and Antibiofouling

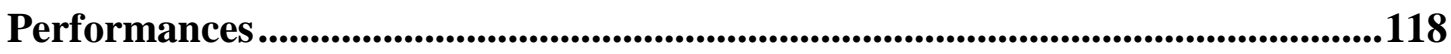

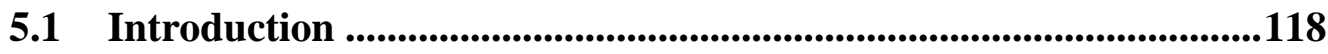


5.2.1 Fabrication of graphene nanosheets (GO), GO-PVDF and GQDsPVDF

5.2.2 Materials characterization. 122

5.2.3 Antimicrobial activities assay 122

5.2.4 Filtration module setup and permeation test 124

5.2.5 Filtration test of GOQDs-PVDF membrane using bacterial feedwater

5.2.6 Oxidative stress of GOQDs-PVDF/GO-PVDF membrane assay.125

5.3 Results and discussion 125

5.3.1 Fabrication and characterization of GOQDs, GO, GOQDs-PVDF and GO-PVDF 125

5.3.2 Membrane properties of pristine PVDF, GO-PVDF and GOQDsPVDF membranes

5.3.3 Antibacterial and antibiofouling performance of GOQDs-PVDF hybrid membrane.

5.3.4 Bactericidal mechanism of GOQDs-PVDF hybrid membrane .... 140

5.4 Summary 144

Chapter 6 General conclusions and recommendations for future research directions. .146

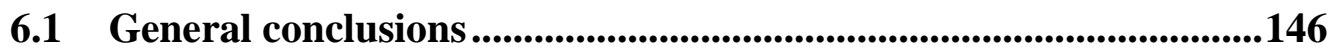

6.2 Recommendations for future research directions 149 


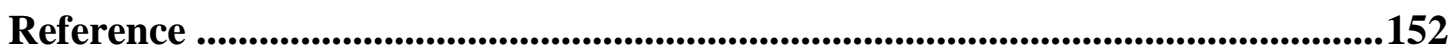




\section{List of abbreviations}

\section{Chemical names}

\begin{tabular}{|c|c|}
\hline 4-NP & 4-nitrophenol \\
\hline 4-AP & 4-aminophenol \\
\hline APTM & (3-aminopropy) trimethoxysilane \\
\hline $\mathrm{CdS}$ & Cadmium sulfide \\
\hline $\mathrm{CdSe}$ & Cadmium selenium \\
\hline $\mathrm{CdTe}$ & Cadmium tellurium \\
\hline DI water & Deionized water \\
\hline ED & Ethylene diamine \\
\hline GO & Graphene Oxide \\
\hline GR & Graphene \\
\hline GOQD & Graphene oxide quantum dots \\
\hline GQD & Graphene quantum dots \\
\hline N-GQDs & Nitrogen-graphene quantum dots \\
\hline $\mathrm{H}_{2} \mathrm{O}_{2}$ & Hydrogen peroxide \\
\hline $\mathrm{HNO}_{3}$ & Nitric acid \\
\hline $\mathrm{HCl}$ & Hydrochloric acid \\
\hline IPA & Iso-propanol \\
\hline $\mathrm{KOH}$ & Potassium hydroxide \\
\hline MWCO & Molecular weight cut-off \\
\hline $\mathrm{NaBH}_{4}$ & Sodium Borohydride \\
\hline $\mathrm{NaOH}$ & Sodium hydroxide \\
\hline $\mathrm{Na}_{2} \mathrm{SO}_{4}$ & Sodium sulfate \\
\hline NHS & n-hydroxysuccinimide \\
\hline PAN & Polyacrylonitrile \\
\hline PEG & Polyethylene glycol \\
\hline $\mathrm{Pt}$ & Platinum \\
\hline PVDF & Polyvinylidene fluoride \\
\hline $\mathrm{TiO}_{2}$ & Titanium dioxide \\
\hline $\mathrm{ZnO}$ & Zinc oxide \\
\hline
\end{tabular}


Physical terms

\begin{tabular}{|l|l|}
\hline atm & Atmosphere \\
\hline C & Capacitance \\
\hline Eg & Bandgap \\
\hline fcc & Face-centered cubic \\
\hline $\mathrm{Hz}$ & Hertz \\
\hline I & Photocurrent density \\
\hline IPCE & Incident photon-to-electron conversion efficiency \\
\hline $\mathrm{J}_{\text {light }}$ & Incident light intensity \\
\hline$N_{D}$ & Carrier density \\
\hline $\mathrm{P}$ & Pressure \\
\hline Voc & Open-circuit voltage \\
\hline $\mathrm{W}$ & Depletion width \\
\hline $\mathrm{Z}$ & Impedance \\
\hline$\alpha$ & Absorption coefficient \\
\hline$\eta$ & Power conversion efficiency \\
\hline$\lambda$ & Wavelength \\
\hline
\end{tabular}


Abbreviations of commonly used terms

\begin{tabular}{|c|c|}
\hline OD & Zero dimensional \\
\hline $1 \mathrm{D}$ & One dimensional \\
\hline $2 \mathrm{D}$ & Two dimensional \\
\hline $3 \mathrm{D}$ & Three dimensional \\
\hline AM & Air mass \\
\hline AFM & Atomic force microscopy \\
\hline c-AFM & Conductive-atomic force microscopy \\
\hline $\mathrm{Ag} / \mathrm{AgCl}$ & Silver chloride electrode \\
\hline AM & Air mass \\
\hline $\mathrm{BE}$ & Binding energy \\
\hline $\mathrm{CA}$ & Contact angle \\
\hline $\mathrm{CB}$ & Conduction band \\
\hline CBD & Chemical bath deposition \\
\hline $\mathrm{CE}$ & Counter electrode \\
\hline CFU & Colony-forming units per millilitre \\
\hline $\mathrm{CV}$ & Cyclic voltammetry \\
\hline DRS & Diffuse reflectance spectra \\
\hline EIS & Electrochemical impedance spectra \\
\hline FESEM & Field emission scanning electron microscope \\
\hline FTO & Fluorine-doped tin oxide \\
\hline FTIR & Fourier transform infrared spectroscopy \\
\hline GC & Gas chromatography \\
\hline HOMO & Highest Occupied Molecular Orbital \\
\hline HRTEM & High resolution transmission electron microscopy \\
\hline ITO & Indium-doped tin oxide \\
\hline $\mathrm{LbL}$ & Layer-by-layer \\
\hline LUMO & Lowest Unoccupied Molecular Orbital \\
\hline LSV & Linear sweep voltametry \\
\hline $\mathrm{M}$ & Metal \\
\hline $\mathrm{NC}$ & Nanocrystal \\
\hline NHE & Normal hydrogen electrode \\
\hline
\end{tabular}




\begin{tabular}{|l|l|}
\hline NR & Nanorod \\
\hline PEC & Photoelectrochemical \\
\hline QD & Quantum dot \\
\hline RE & Reference electrode \\
\hline SAED & Selective area electron diffraction \\
\hline SPR & Surface plasmon resonance \\
\hline VB & Valence band \\
\hline XPS & X-ray photo electron spectroscopy \\
\hline XRD & X-ray diffraction \\
\hline
\end{tabular}




\section{Abstract}

Sustainable clean water supply and efficient energy production remain as major global challenges for humanity despite significant advancement in technology. Graphene quantum dots (GQDs) have fueled tremendous research interest in the past few years owing to their remarkable electronic and physicochemical properties associated with edge and quantum-confinement effects. GQDs feature ultra-small particle size $(<10 \mathrm{~nm})$, affording significantly increased amount of oxygen-containing groups on the planar surface which can serve as highly active catalyst sites. Their exceptionally high conductivity and large specific surface area in conjunction with the existence of various active sites on the planar surface render GQDs a desirable platform for integration with various $0 \mathrm{D}, 1 \mathrm{D}, 2 \mathrm{D}$ and $3 \mathrm{D}$ nanomaterials such that new multifunctional nanohybrids can be realized.

The overall objective of this interdisciplinary research is to utilize and reengineer GQDs as a new platform for nanoscale construction of a series of GQDs-based 0D/0D, 0D/1D and, 0D/2D functional nanocomposites. These new nanohybrids will be endowed with new and enhanced functional properties such as, efficient and multifarious catalysis for advanced sustainable energy conversion, and water remediation applications.

We first perform a controllable structural design comprising of customizable alternate layers of active and functional zero-dimensional nanomaterials (ie. GQDsbased 0D/0D nano-assembly) which were intimately assembled together. This unique highly ordered multilayer configuration is afforded by a judicious layer-by-layer (LbL) assembly strategy, enabling rational and tunable construction of a series of welldefined metal/graphene quantum dots $(\mathrm{M} / \mathrm{GQDs})_{\mathrm{n}}(\mathrm{M}=\mathrm{Au}, \mathrm{Ag}, \mathrm{Pt})$ multilayers. These multilayer thin films demonstrated remarkably efficient and versatile catalytic 
performances toward selective organic transformation of aromatic nitro compounds, electrocatalytic methanol oxidation and light-irradiated photoelectrochemical water splitting at ambient conditions, attributed to the cooperative synergy of metal NCs and GQDs building blocks.

A layer-by-layer (LbL) assembly of highly ordered nitrogen-doped graphene quantum dots (N-GQDs)/ one-dimensional (1D) semiconductor heterostructures (ie. GQDs-based 0D/1D nano-assembly) was successfully fabricated as a high performance photoanode in photoelectrochemical (PEC) hydrogen generation. The deposited amount of N-GQDs and PEC water splitting performances of N-GQDs/ZnO nanowire (NW) heterostructures can be changed by the assembly layer. The integrative roles of nitrogen doping and photosensitization of GQDs in conjunction with intimate interfacial integration between the photoanode components afforded by the LbL assembly strategy were found to collaboratively contribute to enhanced PEC water splitting.

We performed covalent bonding of graphene oxide quantum dots (GOQDs) onto amino modified polyvinylidene fluoride (PVDF) membrane to fabricate a new type of nano-carbon functionalized 2D membrane (ie. GQDs-based 0D/2D nano-assembly) with significantly enhanced antibacterial and antibiofouling porperties. The presence of GOQD coating layer effectively inactivates $E$. coli and $S$. aureus cells, and prevents biofilm formation on the membrane surface, producing excellent antimicrobial activity and potentially antibiofouling capability, more superior than those of previously reported 2D GO sheets and 1D CNTs modified membranes. Furthermore, GOQDs modified membrane possesses satisfying long-term stability and durability owing to the strong covalent interaction between PVDF and GOQDs. 


\section{Chapter 1 Introduction}

\subsection{Background and significance}

Population explosion in tandem with severe environmental issues and upcoming energy crisis has become major challenges to the global community. It is an urgent obligation to develop novel, efficient and multifunctional materials to overcome these challenges. As one of the most common and essential elements in inorganic and organic materials, carbon plays a significant role in our research and industry society. All of carbon allotropes, including football-shaped buckminsterfullerene $\mathrm{C}_{60}$ discovered in 1985,(1) one-dimensional (1D) carbon nanotubes in 1991,(2) and twodimensional (2D) graphene in 2004 (3) have become super stars in materials history. Especially, recent graphene (GR), a single or few layer(s) of carbon atoms in a honeycomb lattice has attracted extensive attention with its extraordinary thermal, electronic and mechanical properties. $(4,5)$

Graphene quantum dots (GQDs), a unique zero-dimensional (0D) carbon nanomaterials, consist of a framework analogous to GR, has emerged and ignited tremendous interests in the past few years owing to its remarkable electronic and physicochemical properties associated with edge and quantum-confinement effects.(6-22) In particular, compared with GR, GQDs is featured by ultra-small particle size $(<10 \mathrm{~nm})$ which affords remarkably more increased oxygen-containing groups on the planar surface which can serve as highly active sites. GQDs exhibited excellent solubility, chemical stability, low cytotoxicity and high luminesce, providing unprecedented opportunities for applications in bioimaging, sensors and optoelectronic devices.(23-32) 
The high conductivity and large specific surface area of GQDs in conjunction with the existence of various active sites on the planar surface render them as a new and desirable platform for integration with various $0 \mathrm{D}, 1 \mathrm{D}$, and 2D nanomaterials. These nanostructured composites are playing critical roles in the development of catalysis in energy conversion, $(33,34)$ such as photovoltaic cells, $(29,34-36)$ rechargeable batteries $(37,38)$ and supercapacitors, $(39,40)$ as well as functional hybrids in environmental applications,(41) such as organic pollutant degradation, $(42,43)$ bactericidal, $(44,45)$ and water treatment.(46-48)

\subsection{Motivations}

The ongoing search for efficient catalysts for organic transformation of key chemical intermediates, as well as for renewable energy conversion systems portrays human's constant struggle in these two fields.(49-53) Recent years have witnessed a flourish of research activities in exploring various nanomaterials for multifarious catalysis targeted for solar energy and chemical-to-energy conversion.(54-56) Among the diverse class of nanomaterials being actively pursued, OD metal nanocrystals (NCs) constitute a pivotal sector of nanomaterials by virtue of its unique surface plasmon resonance (SPR) effect and highly active surface atoms.(57-65) Nevertheless, a quintessential issue in utilizing metal NCs for catalysis is the irreversible aggregation which induces a substantial loss of intrinsic active surface sites and hence inhibited catalytic performances.(66-68) To surmount this obstacle, it is essential to develop an efficacious strategy to preserve the active catalytic surfaces while simultaneously retain the monodispersity of metal NCs.

Producing hydrogen fuel via harvesting sunlight remains as one of the central challenges for solving global energy crisis.(69-74) Photoelectrochemical (PEC) cells 
for solar hydrogen production is a green and promising approach to provide clean energy and thus, have attracted enormous attention since the pioneer work reported by Fujishima and Honda in 1972.(71,75) Thus far, a large variety of nanostructured metal oxides have been explored as photoelectrodes for PEC water splitting, among which 1D semiconductors have been deemed as one of promising electrodes for PEC cells due to their intrinsic structural advantages in comparison with bulk counterparts, such as fast and long-distance electron transport, large surface area, and improved light absorption and scattering capabilities.(76-79) In particular, 1D zinc oxide ( $\mathrm{ZnO})$ nanostructures directly growing on conducting substrates have been extensively utilized for PEC applications due to high electron mobility, environmental friendliness, and comparable $\mathrm{PEC}$ and photocatalytic performances to $\mathrm{TiO}_{2} \cdot(80,81)$ Nevertheless, owing to its relatively large bandgap ( $c a .3 .2 \mathrm{eV}$ ), $\mathrm{ZnO}$ only absorbs ultraviolet light which accounts for merely $\sim 5 \%$ of solar spectrum.(82) It is highly desirable to extend light absorption of $\mathrm{ZnO}$ to visible region for substantial solar energy utilization. Moreover, Layer-by-Layer (LbL) assembly technique, as one of promising bottom-up fabrication strategies, demonstrates remarkable advantages compared with conventional approaches in terms of simplicity and versatility, which furnishes tunable control over micro-structure, thickness, and composition of the assemblies.(83-86) Particularly, intrinsic negatively charged surface and hydrophility of N-GQDs renders it a suitable building block for LbL assembly on the hydrophilic surface of $\mathrm{ZnO} \mathrm{NWs}$ based on electrostatic interaction, thereby resulting in a tailorable nanoarchitecture.

On the other hand, one of the most pervasive challenges afflicting the human race is inadequate access to clean water and sanitation.(87-89) A huge threat to public health worldwide is caused by pathogenic microorganisms in natural and sewage 
water. Membrane technologies are finding wide applications in water treatment,(90) but the growth of pathogenic microorganisms on the membrane surface can produce a fouling layer, i.e., biofilm, and this leads to significant decrease in water flux and increase in energy consumption. Functionalization of water filtration membranes with microorganism resistance property is therefore important in overcoming pathogenic contamination and biofouling.(91,92) Poly(vinylidene fluoride) (PVDF) microfiltration and ultrafiltration membranes, widely used in industrial wastewater and municipal water treatment, possess excellent chemical and thermal stability, high resistance to radiation and strong mechanical property.(93) However, PVDF is intrinsically hydrophobic and therefore easily contaminated by microorganism, resulting in serious membrane fouling.(94) Therefore, new modification strategies of PVDF membrane for effective and long-lasting antibacterial and antibiofouling activities will be highly attractive.

In this $\mathrm{PhD}$ dissertation, GQDs will be investigated as a desirable platform for the integration of the above-mentioned OD metal NCs, 1D semiconductor nanowire/nanotube, and 2D PVDF membrane nanomaterials for advanced energy conversion and environmental applications.

\subsection{Objectives}

The overall objectives of this $\mathrm{PhD}$ program are to engineer GQDs as a new platform for rational construction of a large variety of GQDs-based 0D/0D, 0D/1D, OD/2D functional composite materials at nanoscale, which inspired the fabrication of efficient catalysts with multifarious catalysis for advanced sustainable energy conversion such as solar water splitting, and to construct a multifunctional membrane 
for water and environmental applications with desirable properties such as antibiofouling.

Our specific goals at different stages are:

(1) Design and preparation of GQDs and metal nanocrystals as a 0D/0D nanobuilding block in multilayered architectures towards multifarious catalysis.

To achieve highly efficient and stable catalyst, it is of importance to tailor the nanoarchitecture and morphology of GQDs/metal hybrids to improve the overall performance. For instance, a quintessential issue in utilizing metal NCs for catalysis is the irreversible aggregation which induces a substantial loss of intrinsic active surface sites and hence inhibited catalytic performances. To surmount this obstacle, it is essential to develop an efficacious strategy to preserve the active surfaces while simultaneously retain the monodispersity of metal NCs. An effective strategy to enhance the catalytic activities of metal NCs is to add a uniform support plat via nanoarchitecture design. GQDs, as a OD nanomaterial, is a desirable platform for integration with various metal NCs. In addition, close incorporation of GQDs with metal NCs at nanoscale could provide several merits, including boosting electron transport efficiency of metal NCs, preserving catalytic stability of metal NCs, and achieving cooperative synergy. In order to prepare spatially-ordered 0D/0D multilayer thin films, a facile, green, reproducible, and easily scalable and accessible LbL assembly strategy can be applied based on pronounced electrostatic interaction stemming from the intrinsically oppositely charged surfaces of metal NCs and GQDs.

(2) Fabrication of N-GQDs and 1D semiconductor nanoarchitectures for water splitting under simulated solar light illumination. 
To fabricate N-GQDs without involving any tedious synthetic procedures and harsh experiment conditions, we first develop a rather facile and simple synthesis approach. In this work, the well-defined N-GQDs/1D semiconductor nanohybrid heterostructures were fabricated by LbL assembly strategy. The tunable control over the decoration of N-GQDs on the 1D semiconductors and efficient charge transfer channel for solar hydrogen production performances can be offered. Furthermore, the integrative roles of nitrogen doping and photosensitization of GQDs in conjunction with intimate interfacial integration between the photoanode components were also demonstrated in this stage.

(3) Construction of a stable GOQDs-functionalized membrane for significantly-enhanced bactericidal and antibiofouling applications.

To prepare stable nano-carbon functionalized membrane, we establish a method to achieve covalent bonding of GOQDs onto amino modified PVDF membrane. The GOQD coating layer effectively inactivated the bacteria and prevented the biofilm formation on the membrane surface, producing excellent antimicrobial activity. Such distinctive antimicrobial performance could be ascribed to the unique structure and uniform dispersion of GOQDs, enabling the exposure of a larger fraction of active edges and facilitating the formation of a larger oxidation stress.

\subsection{Organization of the dissertation}

This dissertation is organized as following: 
Chapter 1 provides a general introduction of this dissertation, including the background and significance of GQDs based nanoarchitectures. It also discusses the objective as well as the motivation of this $\mathrm{PhD}$ dissertation.

Chapter 2 surveys recent related research work, which includes an overview of GQDs fabrication methods and GQDs-based 0D/0D, 0D/1D, 0D/2D, and 0D/3D nanoarchitectures, illustrates the various performances, especially in catalysis, hydrogen evolution reaction, oxygen reduction reaction, sensor and some environmental applications.

Chapter 3 reports the GQDs/metal nanocrystals as a 0D/0D nanobuilding block in multilayered architectures towards multifarious catalysis, including selective organic transformation of aromatic nitro compounds, electrocatalytic methanol oxidation and solar light-irradiated PEC water splitting performance.

Chapter 4 discusses the N-GQDs/1D semiconductor heterostructures as a high performance photoanode in PEC hydrogen generation. The rational design of welldefined N-GQDs/1D semiconductor heterostructures with controllable architectures and intimate interfacial contact, at the nanoscale level, was achieved via a facile LbL assembly approach.

Chapter 5 introduces the first covalently-anchored GOQDs-PVDF membrane for environmental applications. The GOQDs-immobilized 2D membrane shows significantly enhanced bactericidal activities and antifouling properties, superior to exiting nano-carbon (such as 1D carbon nanotube, 2D graphene oxide) modified 
polymer membranes, without compromising on the permeation properties of the membrane.

Chapter 6 summarizes the current dissertation with a general conclusion, and provides some recommendation of directions for future research. 


\section{Chapter 2 Literature review}

\subsection{Fabrication methods of GQDs}

In general, the synthetic routes toward GQDs can be divided into two broad groups: bottom-up and top-down approaches. Bottom-up approaches normally involve some organic synthesis by using some small molecules with aromatic structures such as polycyclic aromatic compounds, and the resultant GQDs product can exhibit excellent control of the size distribution as well as the properties. Topdown approaches involve decomposing and exfoliating cheap graphite-based materials in harsh experiment conditions. These approaches normally contain some concentrated acids with strong oxidizing agents in high temperature, resulting that the size distribution and morphology of product cannot be controlled precisely.

\subsubsection{Bottom-up approaches}

GQDs can be assembled with chemical method by small organic molecules or other precursors with comparable morphology of honeycomb carbon structure. Liu's group fabricated mono-dispersed disk-like GQDs by using unsubstituted hexa-perihexabenzocoronene (HBC) as precursors with the treatment of carbonization, oxidization, functionalization, and reduction (Figure 2-1).(95) The synthesized GQDs show uniform distribution with an average size of $60 \mathrm{~nm}$ and a high photoluminescence (PL) efficiency of $3.8 \%$. 


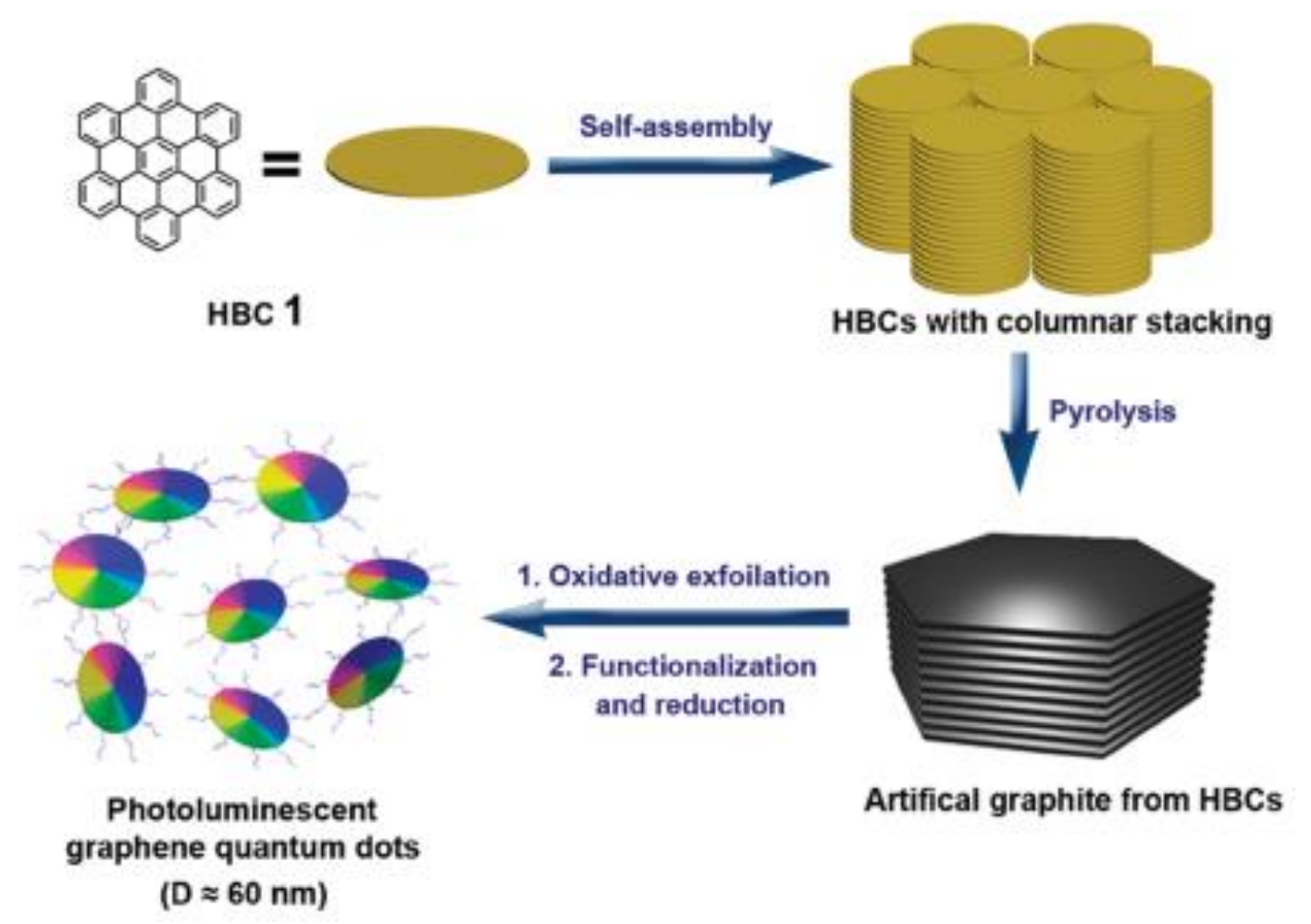

Figure 2-1. Scheme of the sythesis prosess of photoluminescent GQDs by utilizing unsubstituted hexa-peri-hexabenzocoronene (HBC) as carbon precursors.(95) Reproduced with permission from Ref.(95). Copyright 2011 American Chemical Society.

Li's group reported that colloidal GQDs were fabricated via a step-wise organic route.(96) 168, 132, and 137 conjugated carbons group were first synthesized by oxidizing polyphenylene dendritic materials, and then GQDs with tunable size were made up of these conjugated carbons group with the stabilization of 2',4',6'-trialky phenyl reagent. They further synthesized large colloidal GQDs with uniform size by using the trialkyl phenyl groups to attach the quantum dots, preventing the stacking of graphene nanostructure in 3D mode (Figure 2-2), and those large uniform GQDs can be utilized as sensitizer for solar cells.(97) This group also added small benzene derivatives to produce nitrogen-doped GQDs and demonstrated that increasing the nitrogen atoms with a specific bonding configuration, resulting in enhanced ORR performance.(98) 


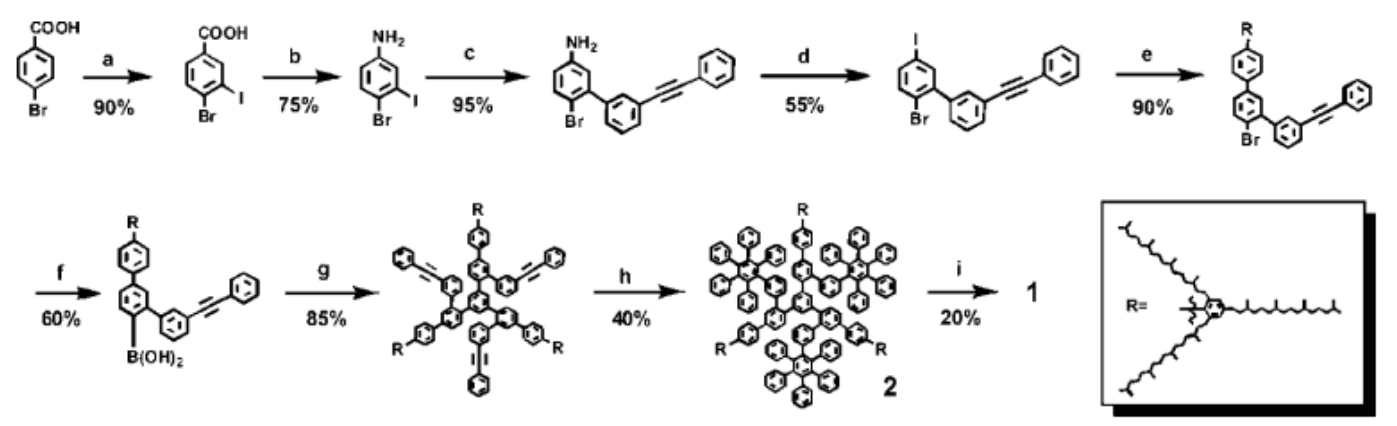

Figure 2-2. Synthesis progress of GQDs. (97) Reproduced with permission from Ref.(97). Copyright 2010 American Chemical Society.

Lu's group prepared GQDs via the ruthenium-catalyzed caged-opening of $\mathrm{C}_{60}$ molecules.(99) $\mathrm{C}_{60}$ molecules make a strong interaction, inducing the surface vacancies on the ruthenium crystal, and subsequently, $\mathrm{C}_{60}$ were embed on the ruthenium surface, producing carbon cluster from the fragmentation of the embedded molecules. The diffusion and aggregation of the resultant carbon clusters form small GQDs. The size and shape of GQDs can be controlled by tuning the annealing temperature.

Wang's group synthesized a high-quality single-crystalline colloidal GQDs with small organic molecules (Pyrene) by hydrothermal method.(100) The resultant GQDs exhibit superior optical properties, such as obvious excitation absorption bands in the whole visible region, efficient excitonic fluorescence, long-term photostability and large molar extinction coefficients.

Heteroatoms can be inherited into GQDs to prepare heteroatom-doping GQDs during bottom-up fabrication progress. For example, Lin's group applied pyrolysis approach to fabricate nitrogen-doped GQDs with citric acid and amino compound.(101) The synthesized GQDs shows a high quantum yield of 59.2\% and can be sensitive during the detection of 2,4,6-trinitrophenol (TNP). Li's group prepared Sulfur doped GQDs by using fructose and sulfuric acid as precursors.(101) 
S, N co-doped GQDs were fabricated by utilizing citric acid as the carbon precursors and thiourea as the sulfur and nitrogen atoms source.(102)

\subsubsection{Top-down approaches}

GQDs can be synthesized by cutting 2D graphene sheets with chemical, electrochemical, or physiochemical means. The majority of top-down approaches can differ in the process they change the graphene-based sheets into quantum dots.

\subsubsection{Hydrothermal/Solvothermal cutting}

A hydrothermal approach was developed to cut the graphene nanosheets into GQDs with blue luminescent.(23) Two main steps were involved, that is, chemical reduction of graphene oxide $(\mathrm{GO})$ to fabricate graphene sheets, and then oxidation of graphene sheets to prepare GQDs in concentrated acid under ultrasonication. Similar to the oxidation of CNTs to shortened CNTs and narrow graphene nanoribbons(103), an unzipping mechanism was proposed for this hydrothermal cutting (Figure 2-3). Under acid oxidization, epoxy groups of graphene sheets tend to become a line on a carbon lattice, leading to a rupture of the $\mathrm{C}-\mathrm{C}$ bonds. The resultant epoxy chain was further oxidized into epoxy pairs and subsequently converted to carbonyl pairs. The group continued to prepare ultrasmall and well-crystallized GQDs with $3.6 \mathrm{~nm}$ average size by utilizing high-temperature chemical cutting method.(104) The resultant GQDs show strong and stable green photoluminescence, demonstrating to serve as a fluorescent nanoprobe for cell imaging. 

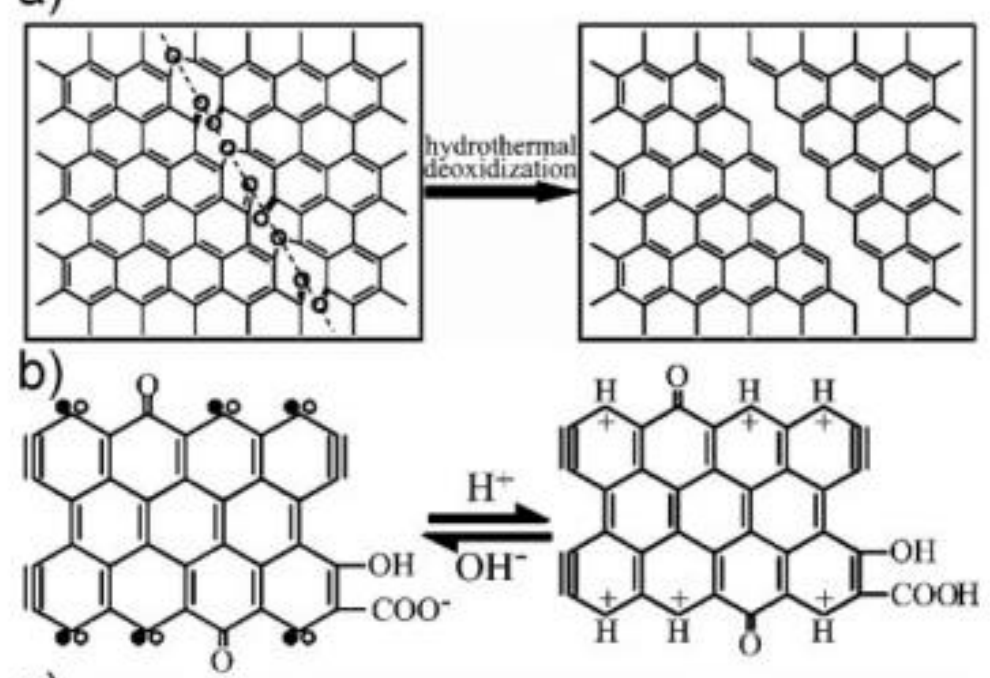

c)

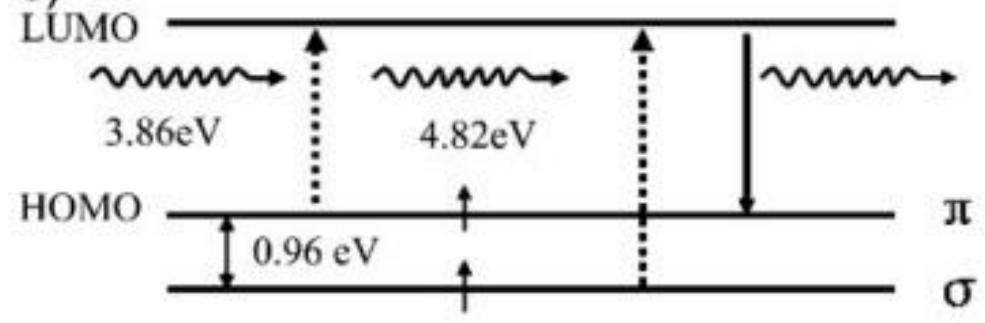

Figure 2-3. Mechanism for the hydrothermal deoxidization of graphene sheets into GQDs based on the report of Pan and the co-workers. (23) Reproduced with permission from Ref.(23). Copyright 2010 Wiley Publishing Group.

A facile hydrazine hydrate reduction of graphene oxide was applied to fabricate GQDs with a surface passivation agent of PEG diamine.(105) The GQDs exhibit bright blue PL under $365 \mathrm{~nm}$ laser and frequency up-converted emission because of the anti-Stokes photoluminescence. They further developed a one-pot hydrothermal reaction to prepare GQDs-PEG, which possessed excellent PL quantum yield and upconversion performances, and larger photocurrent generation capability as compared with GQDs.(106) GQDs fabricated by a two-step chemical process from GO and then functionalized with amine groups exhibit PL shifts and the band gap tuning.(107) The band gap tuning of modified GQDs was also proved by calculations from density functional theory (DFT). 
Peng's group demonstrated a one-step process to synthesize GQDs in large scale amount by using carbon fibers as precursor with acidic etching and exfoliation(Figure 2-4).(108) A range of sizes from 1 to $4 \mathrm{~nm}$ with 1 to 3 graphene layers of GQDs were achieved. By changing the reaction temperature, the PL and bandgap of GQDs can be controlled. They also presented the resultant GQDs exhibit the low cytotoxicity and excellent biocompatibility as well as being an eco-friendly material in bioimaging.
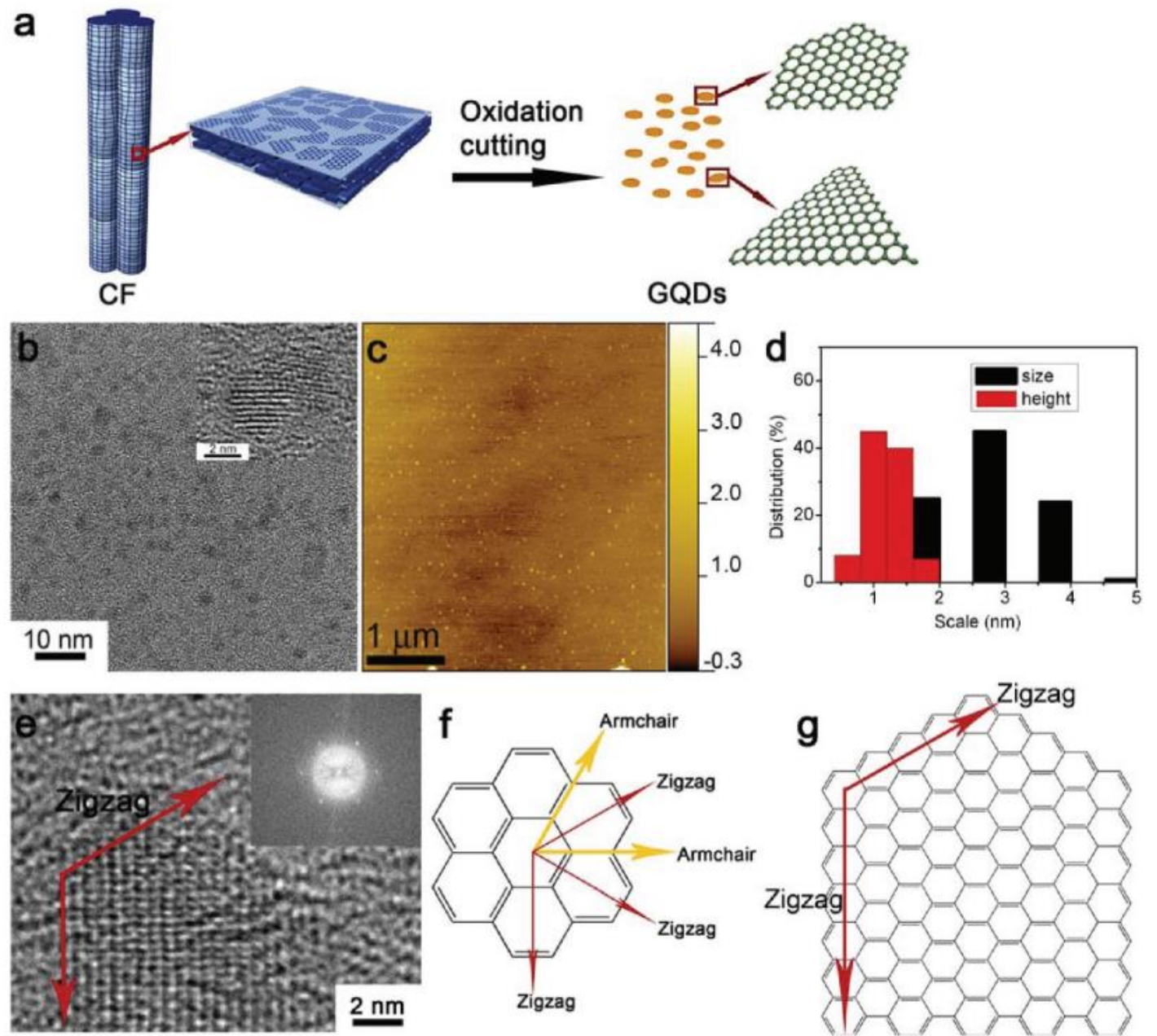

Figure 2-4. Scheme of oxidation cutting carbon fibre into GQDs, and characterization of the synthesized GQDs.(107) Reproduced with permission from Ref.(107). Copyright 2013 American Chemical Society.

Graphite nanoparticles with around $4 \mathrm{~nm}$ diameter were chemically exfoliated to produce homogeneous graphene oxide quantum dots (GOQDs) and GQDs with a 
large amount scale (Figure 2-5).(109) By comparing the optical properties of GOQDs and GQDs, they have demonstrated that GOQDs with oxygen-functional groups exhibit green luminescence, and GQDs with pure $\mathrm{sp}^{2}$ carbon crystalline structure display blue luminescence and a fast recombination lifetime.

a)

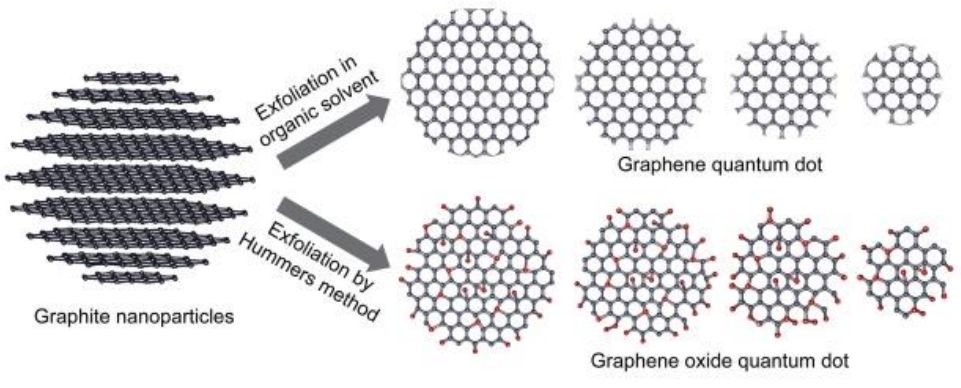

b)
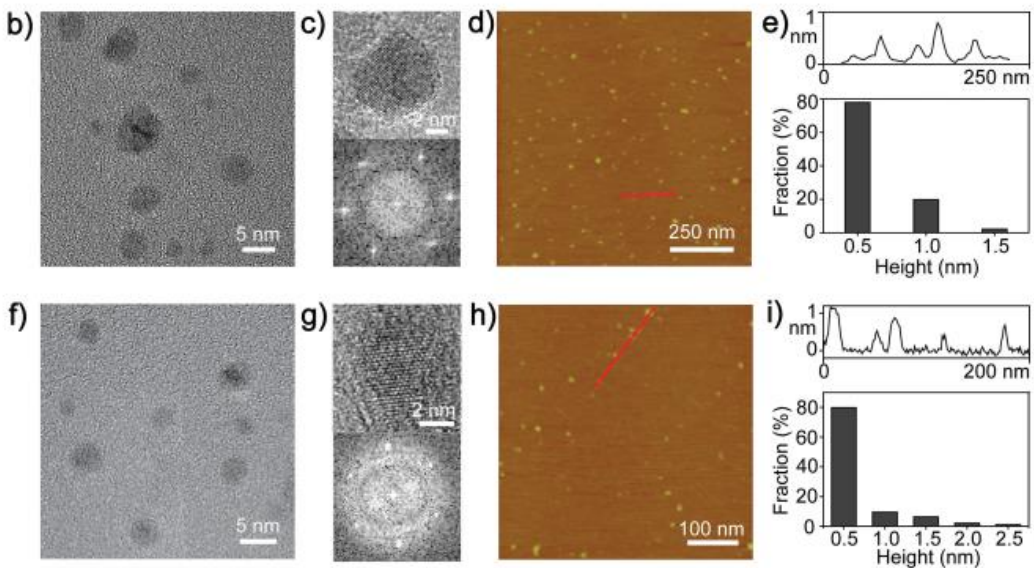

Figure 2-5. (a) Synthesis scheme of GQDs and GOQDs by chemical exfoliation of graphene nanoparitcles. characterization of the synthesized GQDs (b,c,d,e) and GOQDs (f,g,h,i).(109) Reproduced with permission from Ref.(109). Copyright 2013 Wiley Publishing Group.

Carbon black was also used to prepare single-layer and multilayer GQDs with a large yield in a concentrated nitric acid solution by Dong's group.(26) The singlelayer and multilayer GQDs can be further separated by centrifuge in the acidic solution. The prepared multilayer GQDs exhibit a large range of UV-vis absorption, while the synthesized single-layer GQDs display good PL performance and biocompatibility. 
Zhu and co-workers fabricated GQDs with controllable degree of surface oxidation by using a two-step approach with GO as precursors and DMF as solvent. $(25,110)$ The different degrees of oxidation of the synthesized GQDs display tunable PL behaviors.

\subsubsection{Electrochemical Cutting}

Li's group reported to apply electrochemical approach to break down graphene film into functional GQDs with an average size of 3-5 nm.(111) The synthesized GQDs displayed a green luminescence and can retain stable in water solution for several months. Polymer photovoltaic devices by utilizing GQDs as electron acceptor show a conversion efficiency of $1.28 \%$. Zhang's group presented that water-soluble, uniform sized GQDs were prepared by electrochemical exfoliation of graphite.(112) The resultant GQDs were treated by hydrazine reduction and exhibited a strong yellow luminescence with good biocompatibility for stem cell imaging.

\subsubsection{Microwave-assisted Cutting}

Tang's group prepared water-soluble GQDs by a microwave-assisted hydrothermal approach with glucose as precursors.(31) The emission energy of the synthesized GQDs can be up to $4.1 \mathrm{eV}$, which is reported as the largest emission energy among all the solution-based QDs. They also reported that the diameters of GQDs can be controlled from 1.65 to $21 \mathrm{~nm}$ by tuning the heating time.

\subsubsection{Ultrasonic Shearing}

Zhou's group applied a simple ultrasonic method to fabricate GQDs with excitation-independent upconversion and downconversion photoluminescence.(30) The synthesized GQDs were used to decorate rutile and anatase $\mathrm{TiO}_{2}$ for visible-light irradiated degradation of methylene blue (MB). 


\subsubsection{Other Top-down Routs}

Multi-walled carbon nanotube and graphite flakes can be exfoliated and disintegrated into GQDs with lithium/potassium intercalation method by Lin's group.(113) The fabricated GQDs shows an average size of $20 \mathrm{~nm}$ with great luminescent quantum yield and lower oxidation extent. GQDs can be precisely prepared by using high-resolution electron beam lithography method to cut graphene sheets.(14) This approach required a high-precision device and a small amount yield of GQDs can be fabricated. Lee's group applied self-assembled block copolymers (BCP) as an etching mask to fabricate controlled-size and uniform GQDs from chemical vapor deposition (CVD) grown graphene.(114) The morphology and size of GQDs show very uniform with a hexagonal arrangement. Mohanty's group presented that GQDs with different predetermined shapes such as triangle, rectangle, square and ribbon and tailored dimensions were fabricated by diamond-edge-induced nanotomy (nanoscale-cutting) of graphite into graphite nanoblocks and following exfoliation.(115)

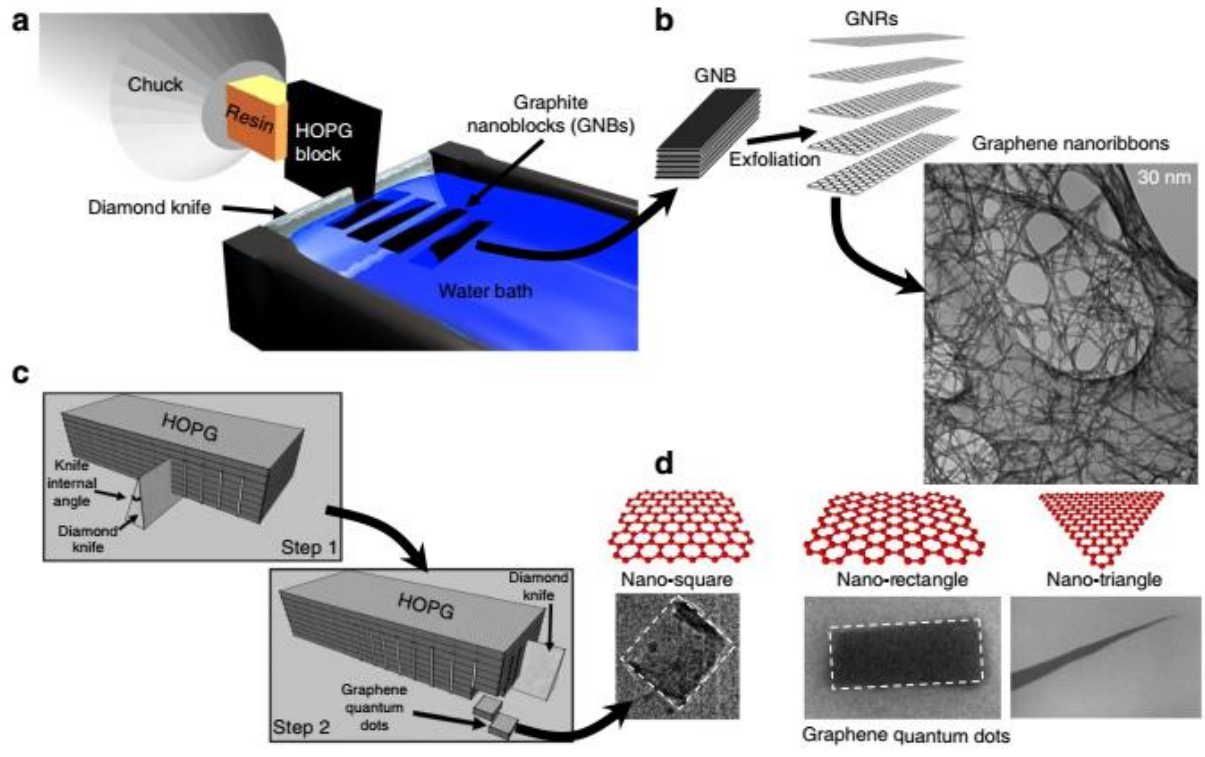

Figure 2-6. Scheme for the GQDs preparation process.(115) Reproduced with permission from Ref.(115). Copyright 2012 Nature Publishing Group. 


\subsection{GQDs-based functionalized materials for multifarious catalysis}

\section{performance}

\subsubsection{GQDs}

Because of the merits (Table 2.1), such as high surface area, low toxicity, water solubility, stable photoluminescence, easy functionalization, excellent chemical and thermal stability, GQDs and GQDs-based materials have been widely applied in multifarious catalysis.

Table 2.1 Comparison about the advantages and disadvantages of GQDs.

\begin{tabular}{|c|c|}
\hline Advantages & Disadvantages \\
\hline Ultra-small particle size $(<10 \mathrm{~nm})$ & \multirow{3}{*}{$\begin{array}{l}\text { Size effects, and quasi-spherical nanoparticles } \\
\text { with sizes below } 10 \mathrm{~nm}(105)\end{array}$} \\
\hline$(11,15,19,23)$ & \\
\hline Water solubility $(21,31)$ & \\
\hline High specific surface area $(8,41)$ & \multirow{2}{*}{$\begin{array}{l}\text { Generally presenting a blue luminescence, not } \\
\text { conducive to biological imaging (227) }\end{array}$} \\
\hline High conductivity $(6,12,36)$ & \\
\hline Stable photoluminescence $(9,17,25)$ & \multirow{2}{*}{$\begin{array}{l}\text { The size distribution and morphology of product } \\
\text { cannot be controlled precisely ( } 24)\end{array}$} \\
\hline $\begin{array}{l}\text { Excellent chemical and thermal stability } \\
\qquad(18,27)\end{array}$ & \\
\hline
\end{tabular}

\subsubsection{Thermal Catalysis}

Carboxyl functionalized GQDs can be applied as an green and efficient acidic catalyst for the coupling reaction of 2-naphthole and benzaldehyde derivatives under solvent-free microwave irradiation conditions.(116) Moreover, carboxyl and nitrite functionalized GQDs prepared by using $\mathrm{NaNO}_{2}$ reagent can be capable to become an effective nitrosonium ion source and reusable catalyst for rapid diazotization, leading to efficient azo-dyes preparation in remarkable yields (Figure 2-7).(117) 


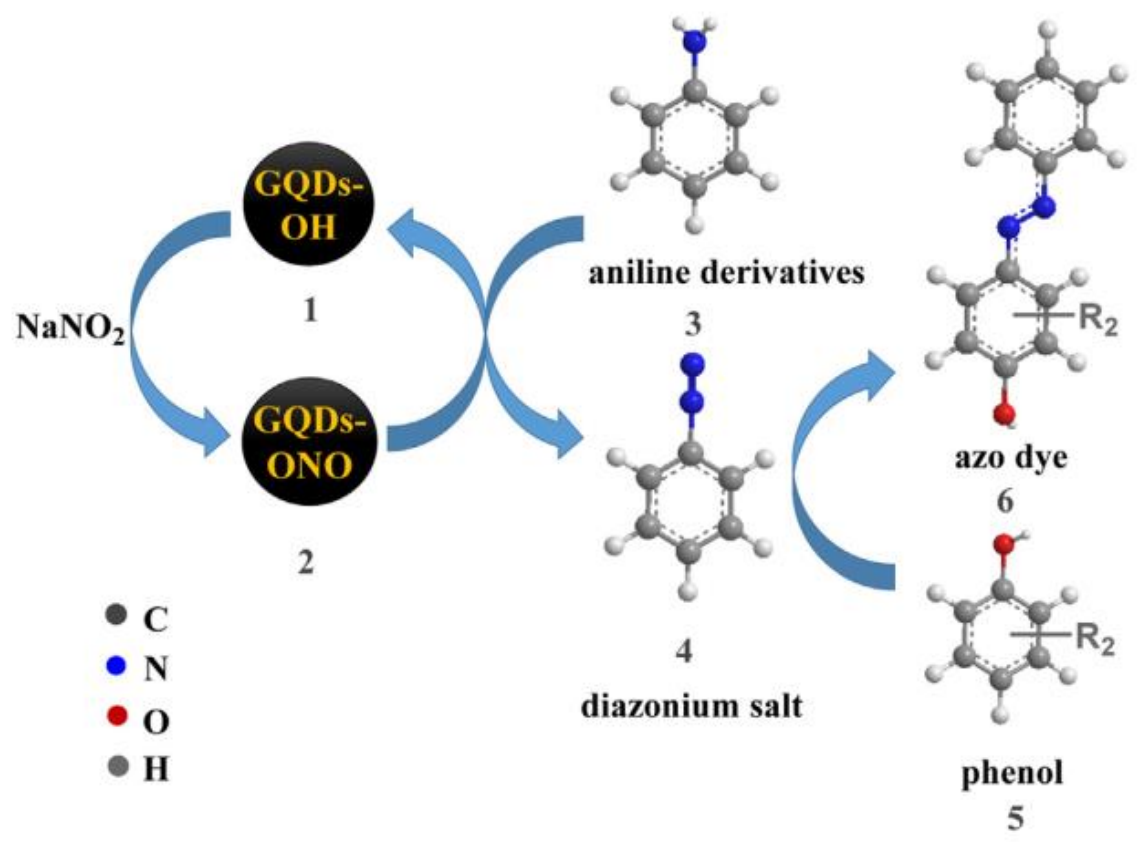

Figure 2-7. Scheme of GQDs-ONO as a nitrosonium precusor and active catalyst for the preparation of azo-dyes.(117) Reproduced with permission from Ref.(117). Copyright 2015 Elsevier Publishing Group.

\subsubsection{Hydrogen Evolution Reaction (HER)}

NGO-QDs fabricated from graphene oxide sheets can be catalyzing overall water splitting under visible-light illumination. The NGO-QDs construction resulted in the formation of p-n type photochemical diodes (Figure 2-8), from which the pand $\mathrm{n}$ - domains were responsible for the production of $\mathrm{H}_{2}$ and $\mathrm{O}_{2}$ gases, respectively.(16,118) This NGO-QDs were further deposited with Pt as cocatalyst, showing high activity in $\mathrm{H}_{2}$ generation with $12.8 \%$ of quantum yield under $420 \mathrm{~nm}$ light irradiation.(119) 


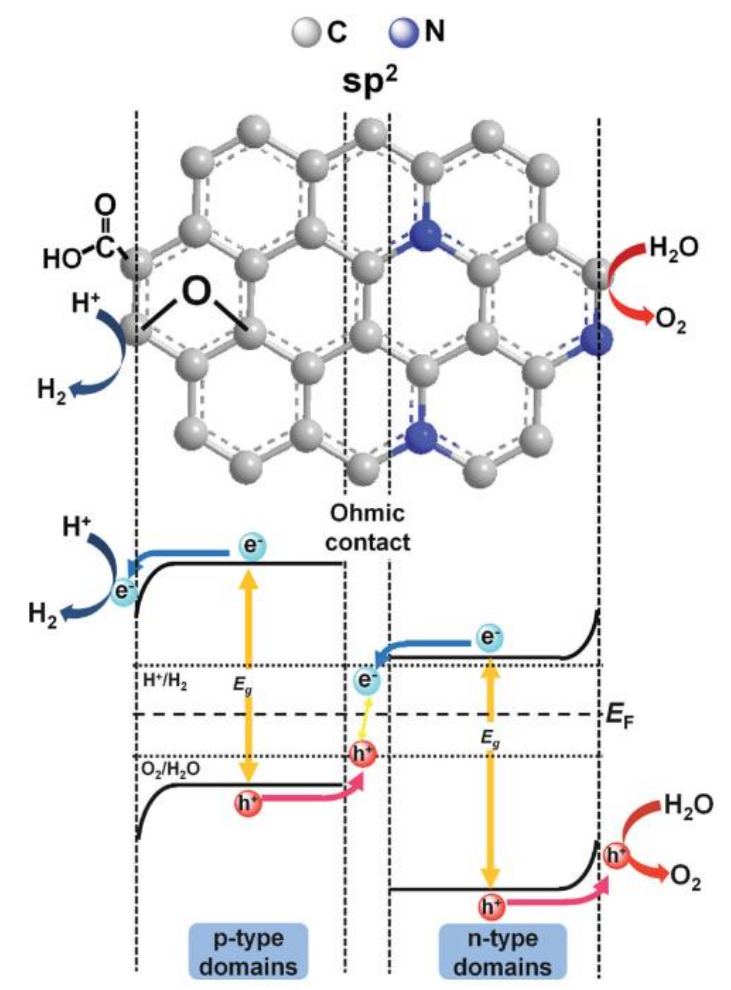

Figure 2-8. The configuration and energy diagram for the NGO-QD photochemical diode.(16) Reproduced in permission from Ref.(16). Copyright 2014 Wiley-VCH.

\subsubsection{Oxygen Reduction Reaction (ORR)}

The cathodic oxygen reduction reaction (ORR) is the rate-limiting factor in metal-air batteries and fuel cells devices. GQDs with heteroatom doping can be transformed into state-of-the-art electrocatalysts for the ORR. Nitrogen-doped GQDs with a large N/C atomic ratio (4.3\%) prepared by a simple electrochemical method exhibited an efficient electrocatalytic performance for the ORR as compared to a commercial Pt/C catalyst.(120) N-doped colloidal GQDs fabricated by a solution chemistry approach demonstrated that nitrogen doping and the size of GQDs can significantly affect their electrocatalytic activity for ORR.(98) First-principles investigation of electrocatalytic activity toward the four-electron ORR in N-GQDs shows that pyridinic and graphitic nitrogen are the most active sites.(121) 
Singly and multiply doped graphene oxide quantum dots (GOQDs) fabricated by a facile electrochemical approach have been reported that B-N codoped materials show a very low overpotential due to the higher concentration of active sites for ORR.(122) With the systematic investigation of density functional calculations about the Gibbs free energy for the transformation of each electron, the selectivity of the reaction depends on the oxidations of the doped GQDs. The as-prepared B-N codoped GQDs can favor the selective production of $\mathrm{H}_{2} \mathrm{O}_{2}$ via a bielectronic reduction route. In comparison, the reduced B-N codoped GQDs follow a 4 ereduction of oxygen to water (Figure 2-9).

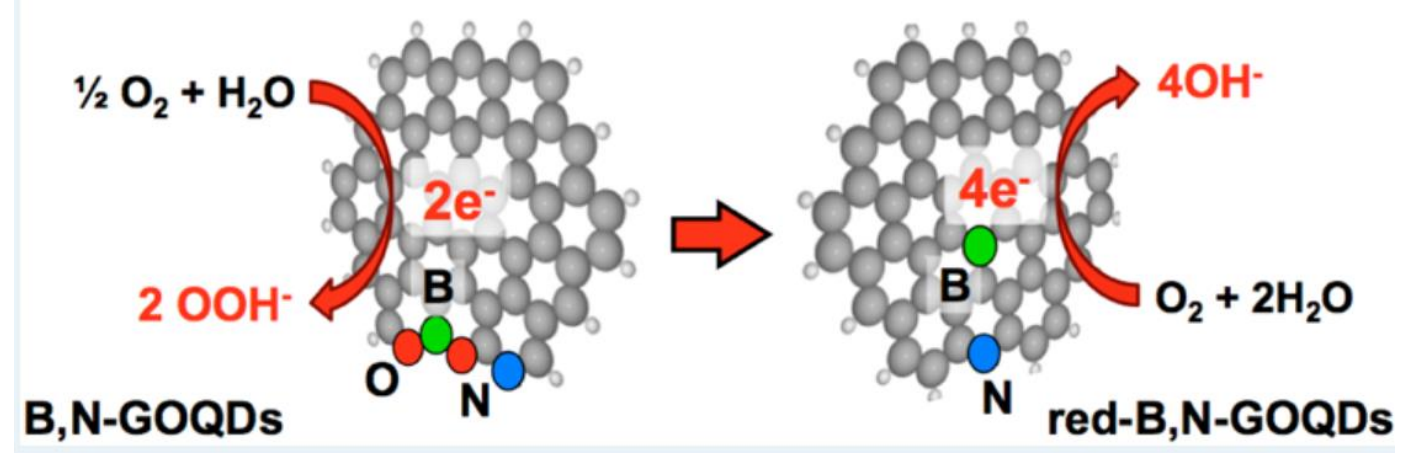

Figure 2-9. Scheme of the selectivity of the reaction controlled by the oxidation states of the dopants in GQDs.(122) Reproduced in permission from Ref.(122). Copyright 2015 American Chemical Society.

\subsubsection{Antibacterial property}

GQDs prepared by rupturing C60 cage can effectively kills Staphylococcus aureus, but not other bacterial, which mis-match the Gaussian curvature of GQDs (Figure 2-10).(123) GQDs fabricated from multi-walled carbon nanotubes (MWCNTs) by using sodium bismuthate $\left(\mathrm{NaBiO}_{3}\right)$ as oxidant possess high and selective toxicity towards bacterial cells but low toxicity to mammalian cells.(124) 


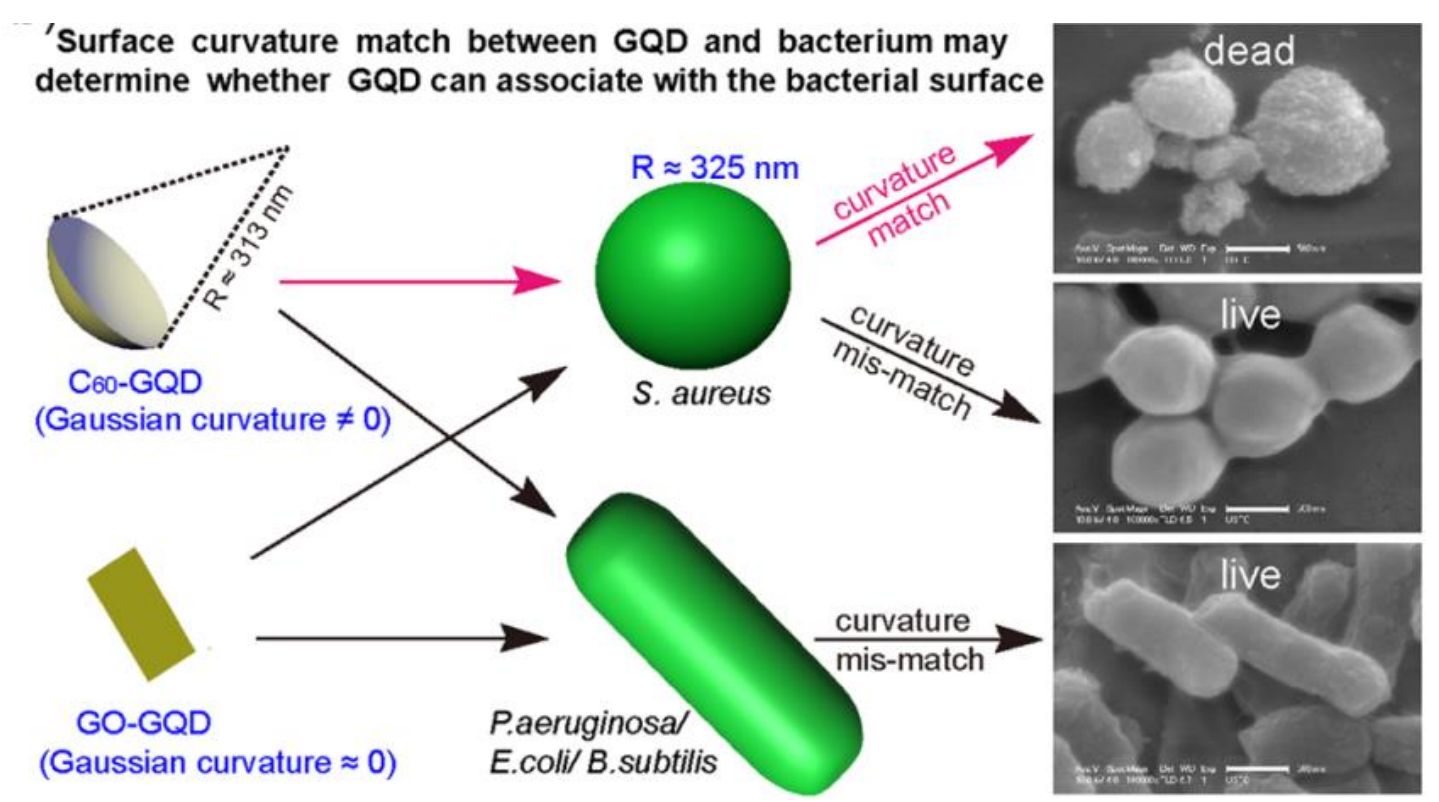

Figure 2-10. Scheme on the surface curvature, which match between a GQD and a trarget bacterium surface.(123) Reproduced in permission from Ref.(123). Copyright 2015 American Chemical Society.

\subsubsection{Sensor}

Through an oxidative ultrasonication of GO, and then reduced and nitrogen doped by reacting with hydrazine, the as-prepared N-GQDs can be utilized to an electrochemical detection of 2,4,6-trinitrotoluene.(125) N-GQDs can significantly accelerate the reaction between 5,10,15,20-tetrakis(1-methyl-4-pyridinio)porphyrin (TMPyP) and cadmium (II) (Figure 2-11), which can be further used for rapid and sensitive determination of metal ions.(126) Nitroaniline can be detected with an electrochemiluminescence system based on N-GQDs and chitosan electrode.(127) N,S-codoped GQDs fabricated by a hydrothermal treatment of citric acid and cysteine can be utilized as a new fluorescent probe for $\mathrm{Au}^{3+}$ ions in real aqueous media.(128) A novel fluorescent platform based on GQDs with intrinsic peroxidasemimicking catalytic activity towards hydroquinone oxidation can be proposed for the sensing of hydroquinone.(129) 


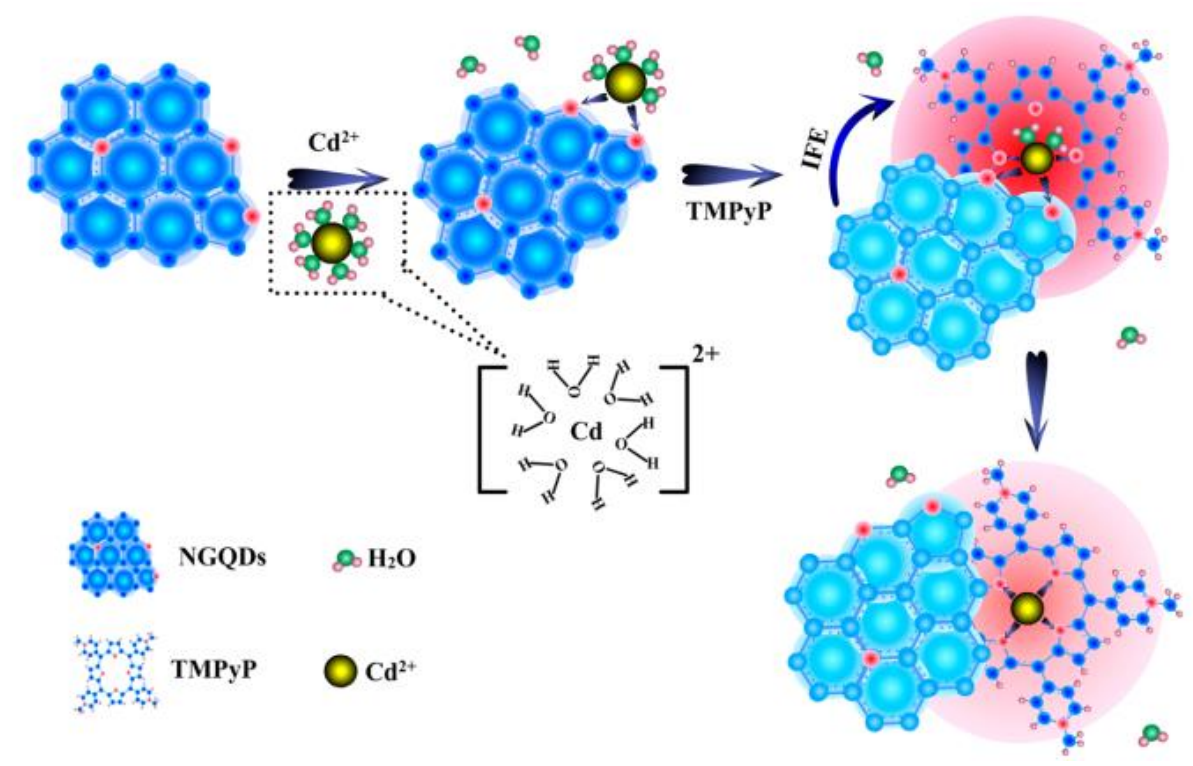

Figure 2-11. Schematic illustrating the catalytic effect of N-GQDs toward the reaction between $\mathrm{Cd}^{2+}$ and TMPyP.(126) Reproduced in permission from Ref.(126). Copyright 2015 American Chemcial Society.

\subsubsection{GQDs-based 0 D materials}

\subsubsection{Thermal Catalyst}

Due to their ultra-small sizes, high surface area and attached functional groups, GQDs can be easily composited with various nanoparticles. GQDs and $\mathrm{Fe}_{3} \mathrm{O}_{4}$ nanoparticles $\left(\mathrm{GQDs} / \mathrm{Fe}_{3} \mathrm{O}_{4}\right)$ synthesized by a one-step co-precipitation method showed super peroxidase-like activities with a higher stability and reusability, as well as an effective catalyst for the removal of phenolic compounds from solutions, which is ascribed to the excellent physicochemical properties of GQDs and the synergistic cooperation between GQDs and $\mathrm{Fe}_{3} \mathrm{O}_{4}$ (Figure 2-12).(130) 


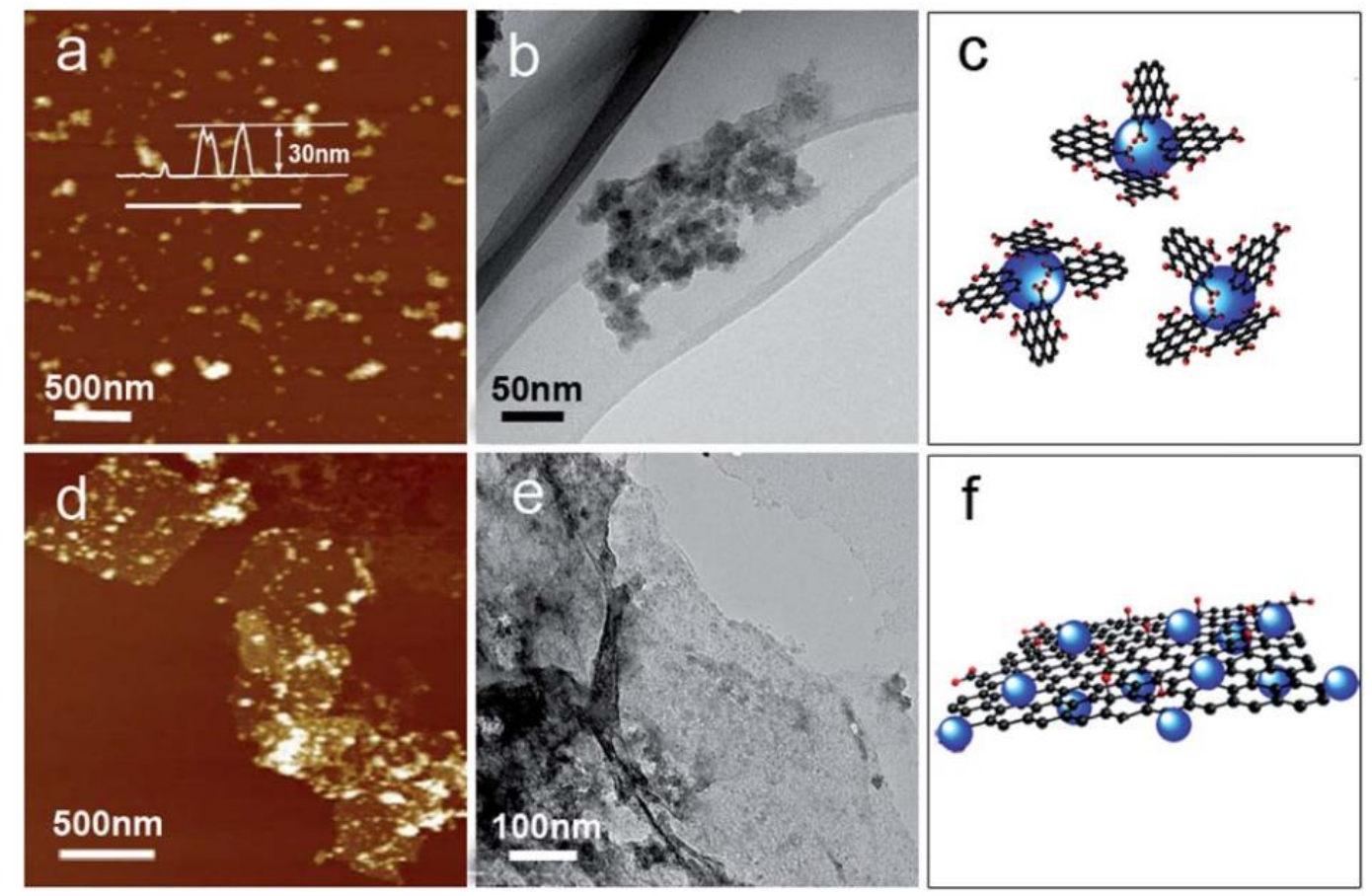

Figure 2-12. AFM images, TEM images, and schematic illumination of the GQDs/ $\mathrm{Fe}_{3} \mathrm{O}_{4}(\mathrm{a}, \mathrm{b}, \mathrm{c})$ and $\mathrm{GO} / \mathrm{Fe}_{3} \mathrm{O}_{4}(\mathrm{~d}, \mathrm{e}, \mathrm{f}) .(130)$ Reproduced in permission from Ref.(130). Copyright 2014 Royal Society of Chemistry.

Au/GQDs composites can be used as catalysts to oxidize veratryl alcohol to veratric acid or veratryl aldehyde by $\mathrm{H}_{2} \mathrm{O}_{2}$ with extraordinary selectivity and conversion efficiency (Figure 2-13a).(131) Ag@GQDs hybrid showed remarkable photothermal effect and catalytic activity for 4-nitrophenol (4-NP) reduction, which is ascribed to the synergistic interaction of GQDs and Ag, and the SPR-mediated photothermal local heating of Ag (Figure 2-13c).(132) Pd supported on N-GQDs was applied as a catalyst in the reduction reaction of nitroaromatics using $\mathrm{NaBH}_{4}$, exhibiting green reduction activity.(133) 
(a)
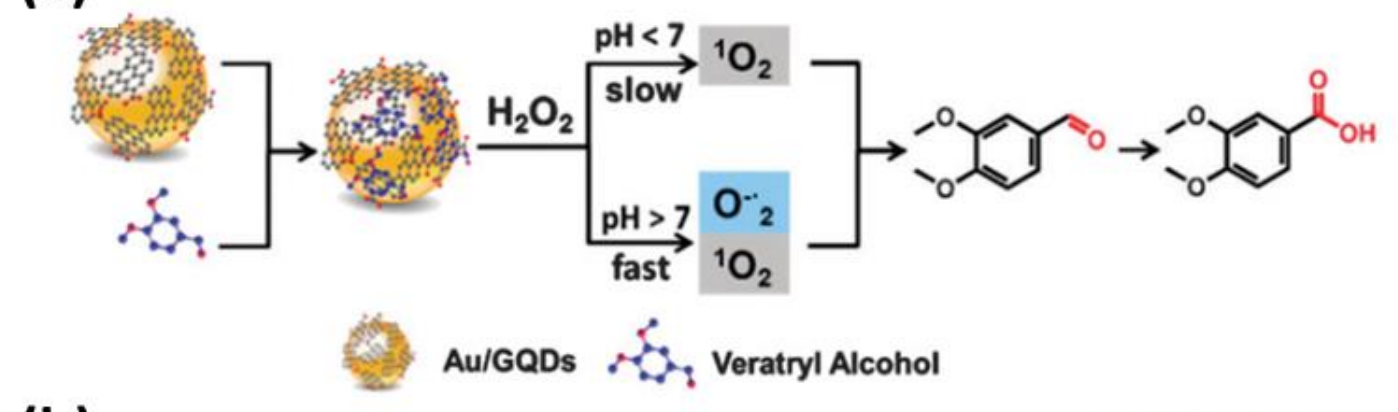

(b)

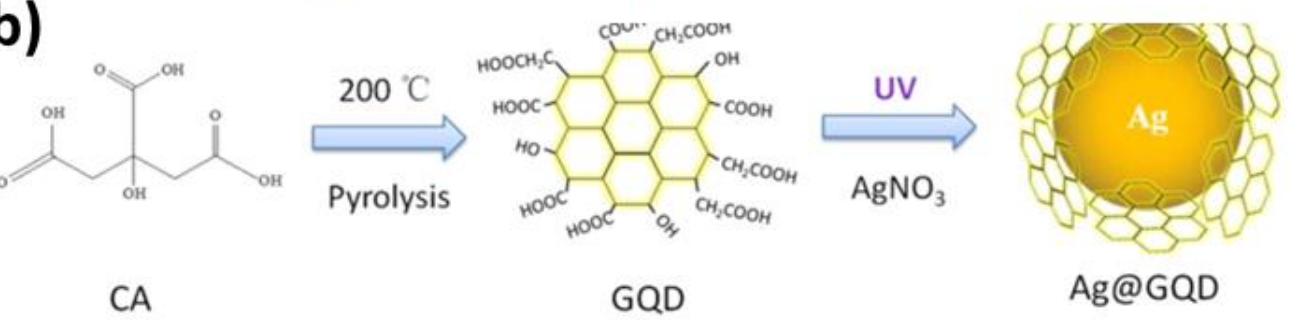

(c)
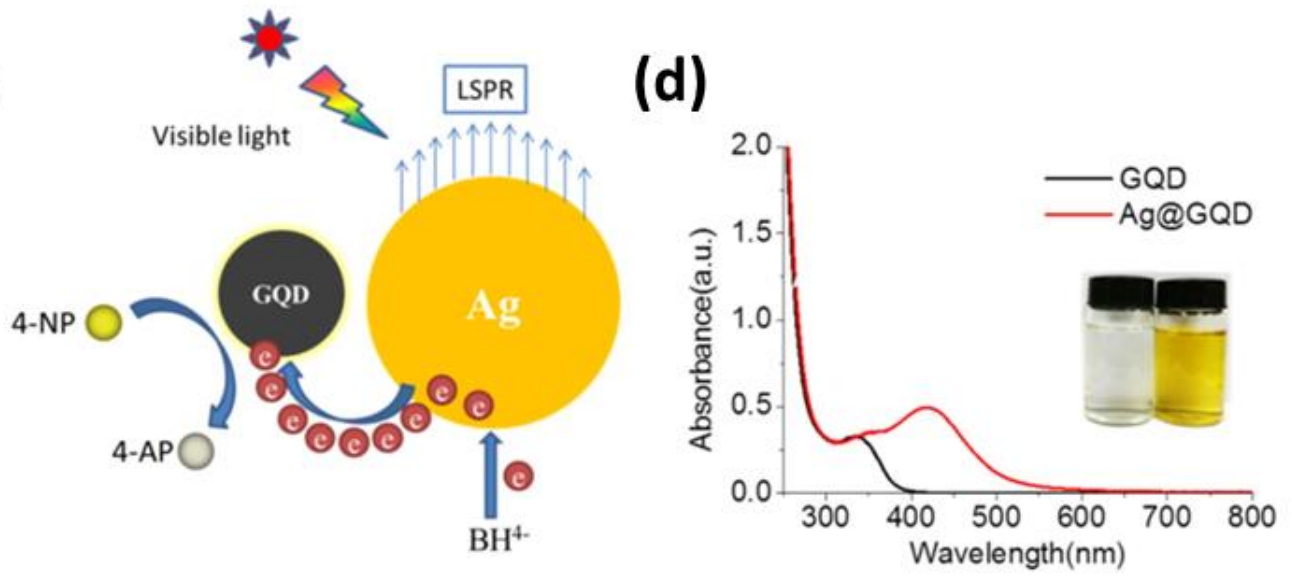

Figure 2-13. (a) Scheme of the mechanism for veratryl alcohol oxdiation with Au/GQDs catalysts.(131) Reproduced in permission from Ref.(131). Copyright 2016 Royal Society of Chemistry. (b) Scheme of the fabrication of Ag@GQDs composite. (c) The mechanism of the conversion of 4-NP to 4-AP catalyzed by Ag@GQDs hybrid. (d) UV-vis spectra of GQDs and Ag@GQDs.(132) Reproduced in permission from Ref.(132). Copyright 2016 IOP Publishing Group.

\subsubsection{HER}

GQDs-based OD composite can be applied as photocatalysts for hydrogen evolution. A novel metal-free $\mathrm{N}-\mathrm{GQDs} / \mathrm{g}-\mathrm{C}_{3} \mathrm{~N}_{4}$ catalyst shows much better photocatalytic performances for $\mathrm{H}_{2}$ evolution from water splitting under visible light irradiation than that of $\mathrm{g}_{-} \mathrm{C}_{3} \mathrm{~N}_{4}$ owing to the unique and multiple roles of $\mathrm{N}$ - 
GQDs.(134) NGQDs- $\mathrm{ZnNb}_{2} \mathrm{O}_{6} / \mathrm{g}-\mathrm{C}_{3} \mathrm{~N}_{4}$ heterostructures with good favorable light harvesting and charge separation showed high hydrogen evolution rate $\left(340.9 \mu \mathrm{mol} \mathrm{h}{ }^{-}\right.$ ${ }^{1} \mathrm{~g}^{-1}$ ) under visible light irradiation, which can be ascribed to the cooperative effects of NGQDs and heterojunction structure (leading to generate more electron-hole pairs and accelerate the interfacial charge separation).(135)

$\mathrm{Yu}$ and co-workers reported that GQDs decorated on (100) faceted anatase $\mathrm{TiO}_{2}$ shows 8 times higher hydrogen evolution rate than that of pure (100) $\mathrm{TiO}_{2}$, which may originate from a higher charge separation efficiency.(136) In addition, Min's group presented that GQDs coupled $\mathrm{TiO}_{2}$ nanoparticles $\left(\mathrm{GQDs} / \mathrm{TiO}_{2}\right)$ photocatalysts demonstrated highly active photocatalytic activity towards the $\mathrm{H}_{2}$ evolution reaction, resulting in 7 and 3 times higher $\mathrm{H}_{2}$ evolution efficiency and photocurrent response than $\mathrm{TiO}_{2}$ NPs alone, respectively.(137) It has demonstrated that GQDs possess effective photosensitization to maximize light harvest (Figure 2-14a), and act as an electron acceptor to enhance charge separation rate and a reaction center to facilitate HER (Figure 2-14b). 

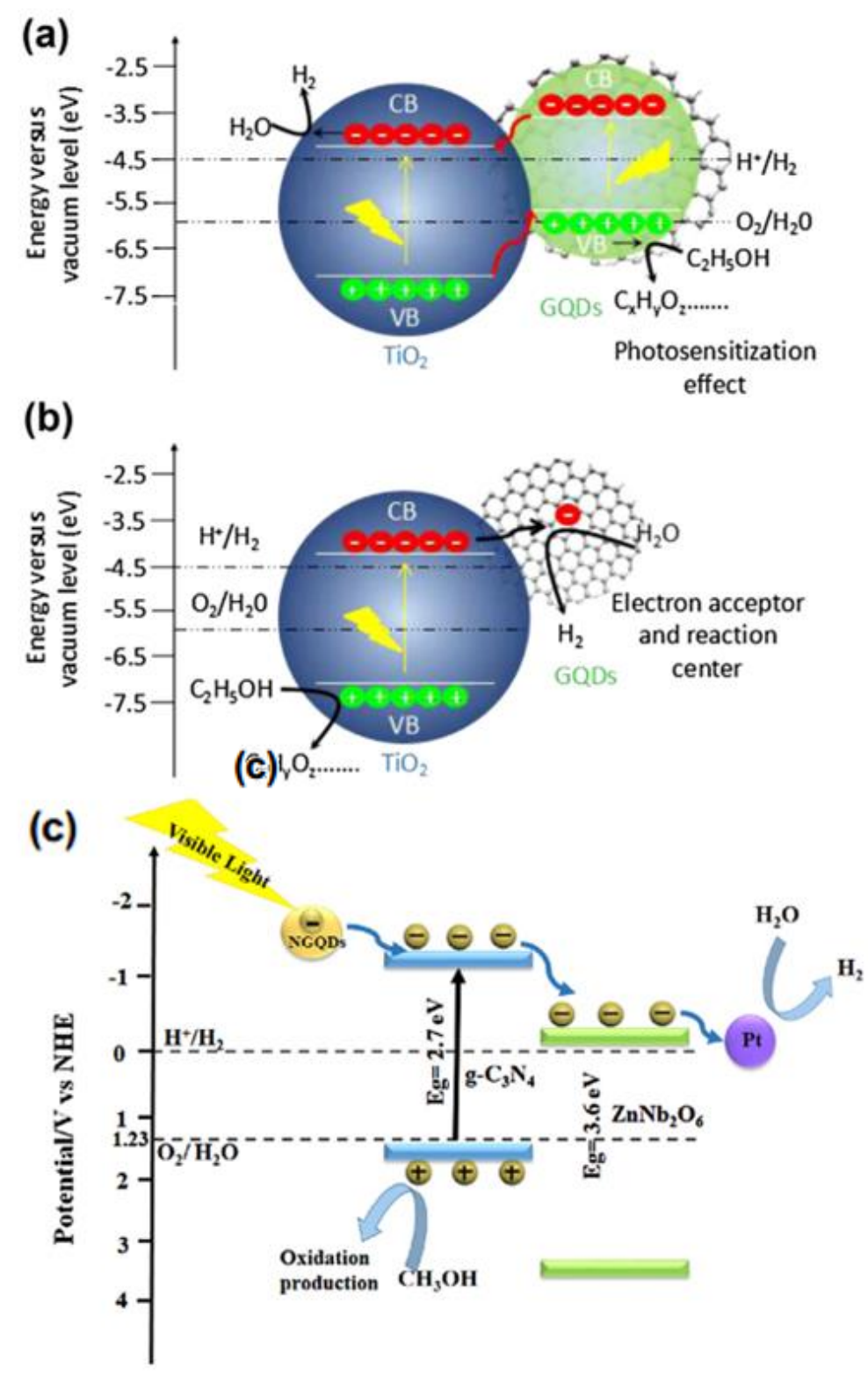

Figure 2-14. Scheme of photocatalytic mechanism for photocatalytic activity of $\mathrm{TiO}_{2}$ /GQDs (a,b). GQDs act as (a) photosensitizers and (b) an elector acceptor and reaction center.(137) Reproduced in permission from Ref.(137). Copyright 2017 Elsevier Publishing Group. (c) Scheme of photocatalytic mechanism for enhanced photocatlytic activity of NGQDs- $\mathrm{ZnNb}_{2} \mathrm{O}_{6} / \mathrm{g}-\mathrm{C}_{3} \mathrm{~N}_{4 \cdot} \cdot(136)$ Reproduced in permission from Ref.(136). Copyright 2017 Royal Society of Chemistry.

\subsubsection{ORR}

GQDs synthesized by etching carbon fibers can be utilized as supporting substrates for platinum $(\mathrm{Pt})$ nanoparticles. Pt/GQDs catalysts displayed a remarkably enhanced electrocatalytic activity with a high positive onset potential $(+1.05 \mathrm{~V})$ 
(Figure 2-15a), excellent specific activity and stability as compared to commercial Pt/C.(138) PtCu@GQDs capsules perform remarkably enhanced catalytic activity in ORR with a two times higher mass activity and a $40 \mathrm{mV}$ more positive onset potential than that of commercial Pt/C (Figure 2-15b,c).(139)

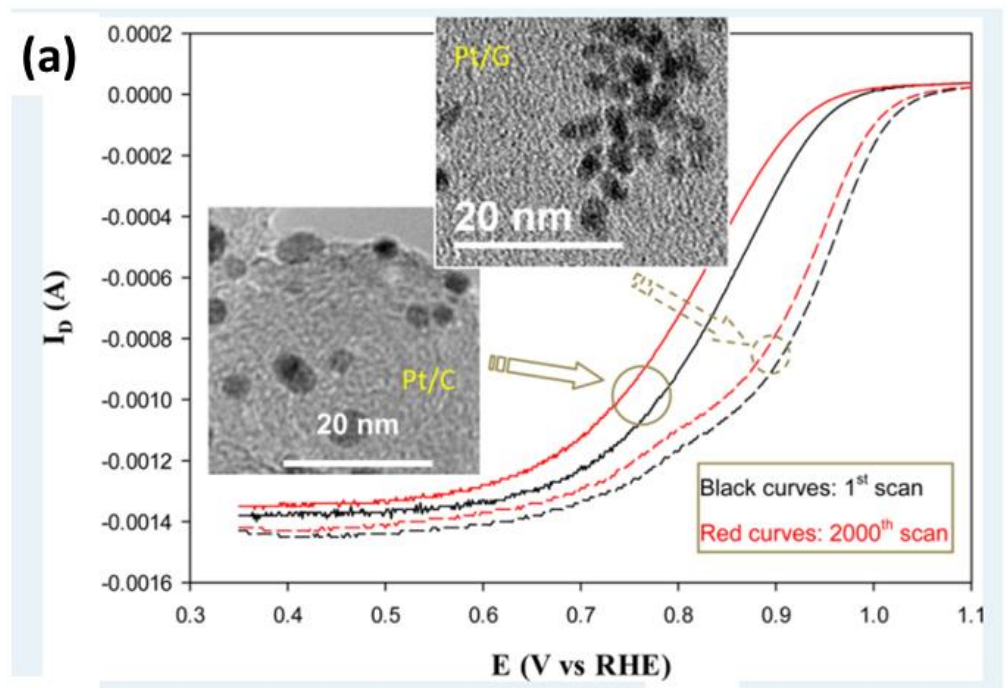

(b)

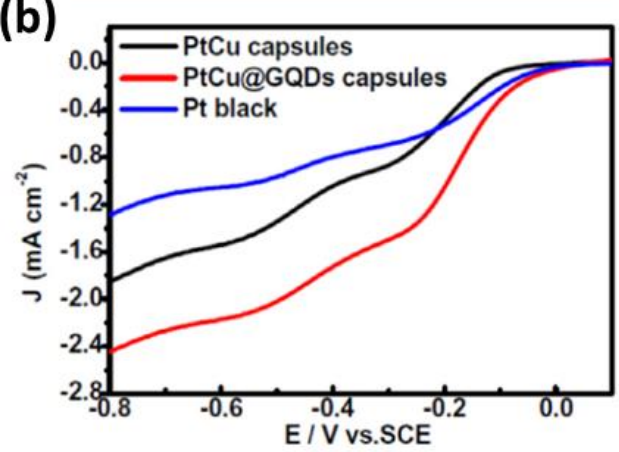

(c)

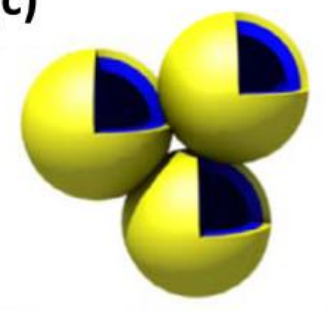

PtCu@GQDs capsules

Figure 2-15. (a) Polarization curves for ORR performance by $\mathrm{Pt} / \mathrm{C}$ (solid curves) and Pt/G (dashed curves) before (red curves) and after (red curves) 2000 cycles.(138) Reproduced in permission from Ref.(138). Copyright 2013 American Chemical Society. (b) Catalytic activity towards electrochemical oxygen reduction of $\mathrm{PtCu} @$ GQDs capsules, PtCu capsules, Pt black in KOH aqueous solution.(139) Reproduced in permission from Ref.(139). Copyright 2014 Elsevier Publishing Group.

Pd/GQDs nanocomposites with optimal interactions between Pd and GQDs (synthesized via thermolytic reduction of metal salts) possess excellent electrocatalytic activity for ORR in alkaline media.(140) Furthermore, Pd NPs supported on Nitrogen-doped GQDs (N-GQDs) showed remarkably electrocatalytic 
activity toward ORR, with a top specific activity in compared with Pd-GQDs, which is accounted for the enhanced electron withdrawing effects of the pyrrolic nitrogen centers that manipulated the electronic interactions between palladium and oxygen intermediates.(141) GQDs-supported copper nanoparticles (Cu/GQDs) fabricated by refluxing of copper compounds and GQDs exhibited apparent electrocatalytic activity towards ORR within the context of onset potential, kinetic current density and number of electron transfer.(142) Sulfur-doped graphitic carbon nitride $\left(\mathrm{s}-\mathrm{g}-\mathrm{C}_{3} \mathrm{~N}_{4}\right)$ modified with GQDs fabricated by a one-step hydrothermal method exhibited excellent enhanced catalytic performance in the ORR, which performs much better than the bare s-g- $\mathrm{C}_{3} \mathrm{~N}_{4}$ and GQDs, even comparable to graphene.(143)

\subsubsection{Degradation}

For the photo-degradation of organic pollutants (methylene blue), the photocatalytic rate of rutile $\mathrm{TiO}_{2}$ complex decorated with GQDs (rutile $\mathrm{TiO}_{2} / \mathrm{GQDs}$ ) shows ca. 9 times higher than that of anatase $\mathrm{TiO}_{2} / \mathrm{GQDs}$ complex under visible light irradiation.(30) GQDs- $\mathrm{TiO}_{2}$ photocatalyst with bandgap narrowing mechanism demonstrated that the larger the size range of GQDs, the narrower the bandgap of the heterostructure composites, the further the absorption edge of the heterostructure composite extended to the visible light period, thus improving the electron-hole separation during the visible light-irradiated photo-degradation of an organic dye methylene blue (MB).(144) S,N co-doped $\mathrm{GQDs} / \mathrm{TiO}_{2}$ composites demonstrated excellent visible light-irradiated photocatalytic performance for degrading rhodamine $\mathrm{B}(\mathrm{RhB})$, of which photocatalytic rate is 3 times higher than that of $\mathrm{N}$ GQDs/TiO $\cdot(145)$

Graphite carbon nitride composited with S,N co-doped GQDs prepared by a physical method exhibit high photocatalytic activity for degrading RhB under visible- 
light irradiation, which is attributed to the superior separation of photogenerated holes and electrons.(124) GQDs decorated $\mathrm{ZnO}$ and $\mathrm{Cu}$ composite fabricated by a facile spin-coating and annealing treatment displays remarkable improvements in UV lightirradiated photodegradation of $\mathrm{RhB}$, which is attributed to the synergistic interactions of $\mathrm{Cu}$ NPs and GQDs.(146) Leaf-templated $\mathrm{ZnO}$ nanoparticles combined with S,Ncodoped GQDs (L-ZnO/S,N-GQDs) composites show high photocatalytic performance for the photodegradation of RhB under visible light illumination, owing to the highly effective charge separation and high surface area with efficient capture of visible light (Figure 2-16a).(147) The ternary photocatalyst Ag-GQDs-ZnO displayed excellent visible-light photocatalytic performance in the visible-light irradiated degradation of $\mathrm{RhB},(148)$ which was far better than that of bare $\mathrm{ZnO}$ and binary hybrids (GQDs-ZnO or Ag-ZnO) (Figure 2-16b). It can be attributed to the synergistic cooperation of the Ag NPs and GQDs. 

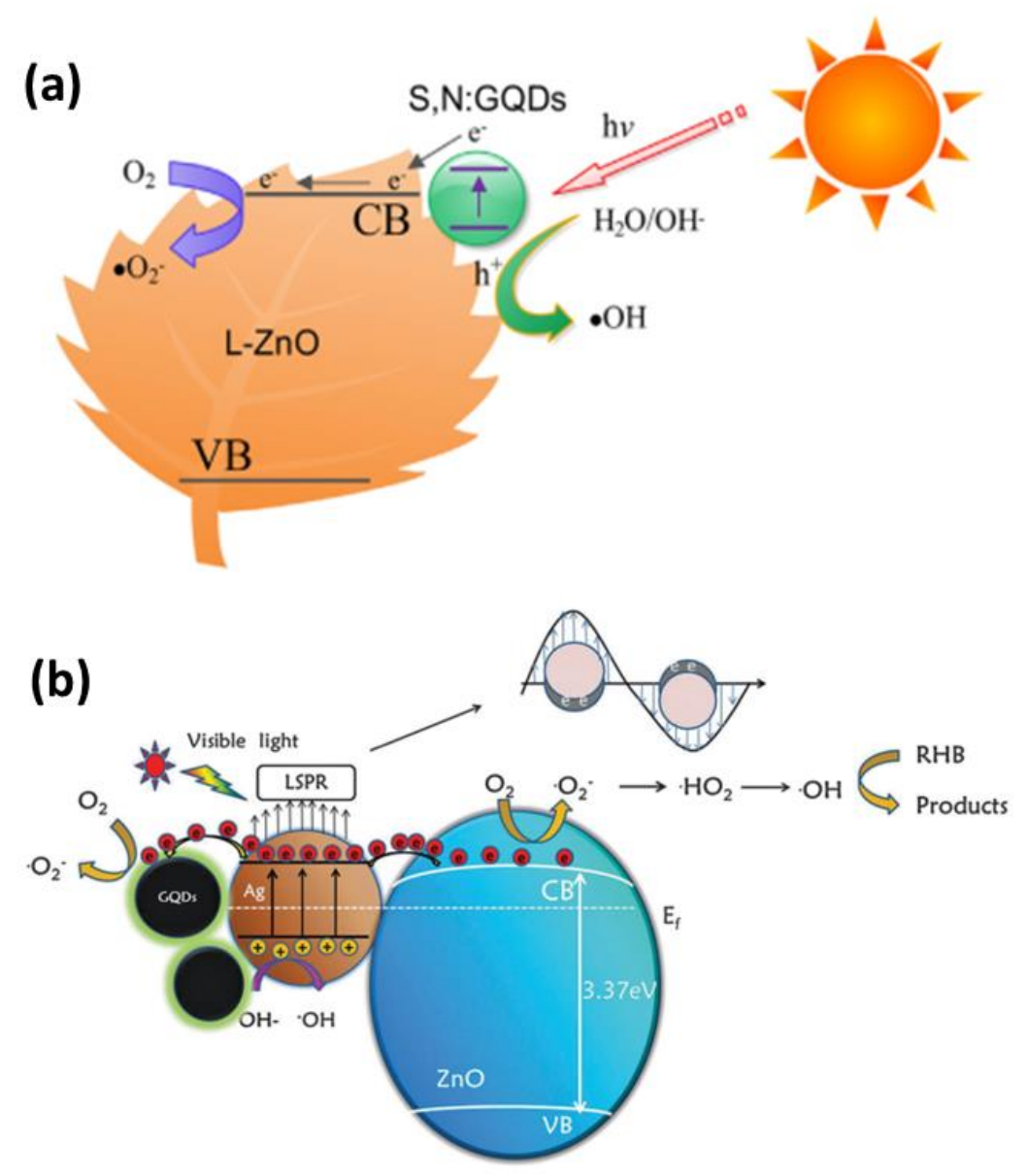

Figure 2-16. (a) Scheme of the working mechanism of L-ZnO/S,N:GQDs.(147) Reproduced in permission from Ref.(147). Copyright 2017 Elsevier Publishing Group. (b) Scheme of the charge transfer mechanism of Ag-GQDs-ZnO ternary composite film.(148) Reproduced in permission from Ref.(148). Copyright 2015 Royal Chemical Society.

\subsubsection{Sensor or Detection}

GQDs can be utilized as a preferable supporting material for hybrid catalysts, due to its many splendid properties, such as high electrical conductivity, large surface area, high stability and good photoluminescence. Au-GQDs modified glass carbon electrode for catechol sensing demonstrate the synergist cooperation between $\mathrm{Au}$ and GQDs can increase specific surface area and enhance catalytic and electronic properties of glass carbon electrode.(149) Au NPs-N-GQDs fabricated by in situ growth display high selectivity and sensitivity for electrochemical detection of $\mathrm{H}_{2} \mathrm{O}_{2}$ 
with a low detection limit of $0.12 \mu \mathrm{M}$.(150) Ag NPs-decorated with GQDs demonstrated a rapid and label-free detection of biothiols and $\mathrm{Ag}^{+}$with high sensitivity and selectivity, which is due to quenching the fluorescence of GQDs and the formation of $\mathrm{Ag}-\mathrm{S}$ bonds.(151)

\subsubsection{GQDs-based 1 D materials}

\subsubsection{HER}

GQDs-modified 1D materials have been widely used in photocatalysis and photoelectrocatalysis. GQDs modified $\mathrm{ZnO}$ nanowire arrays provides a favorable efficient photoelectrode for PEC water splitting with enhanced visible-light absorption, which is due to the promising energy bandgaps between $\mathrm{ZnO}$ and GQDs that lead to transport charge for efficient electrons-holes separation (Figure 217a,b).(152) The photocatalytic activities for hydrogen production of GQDs modified $\mathrm{TiO}_{2}$ nanotube arrays $\left(\mathrm{GQDs} / \mathrm{TiO}_{2}-\mathrm{NA}\right)$ could be remarkably enhanced by loading GQDs onto the catalysts due to weak light-filtering effect and the promotional charge separation and transfer effect of GQDs.(153) Micropatterned $\mathrm{TiO}_{2}$ nanorods $\left(\mathrm{TiO}_{2} \mathrm{NRs}\right)$ decorated with GQDs achieves a high photocurrent density $\left(2.92 \mathrm{~mA} \mathrm{~cm}^{-2}\right)$ under simulated solar light irradiation, and a large incidentphoton-to-current-conversion efficiency (IPCE) value (72\%) at $370 \mathrm{~nm} .(154)$ The sensitization of GQDs on $\mathrm{TiO}_{2}$ hollow nanowires shows enhancing the light harvesting efficiency and the catalytic performance for water photo-oxidation, where GQDs perform as photosensitizers or electron transporter.(155) 

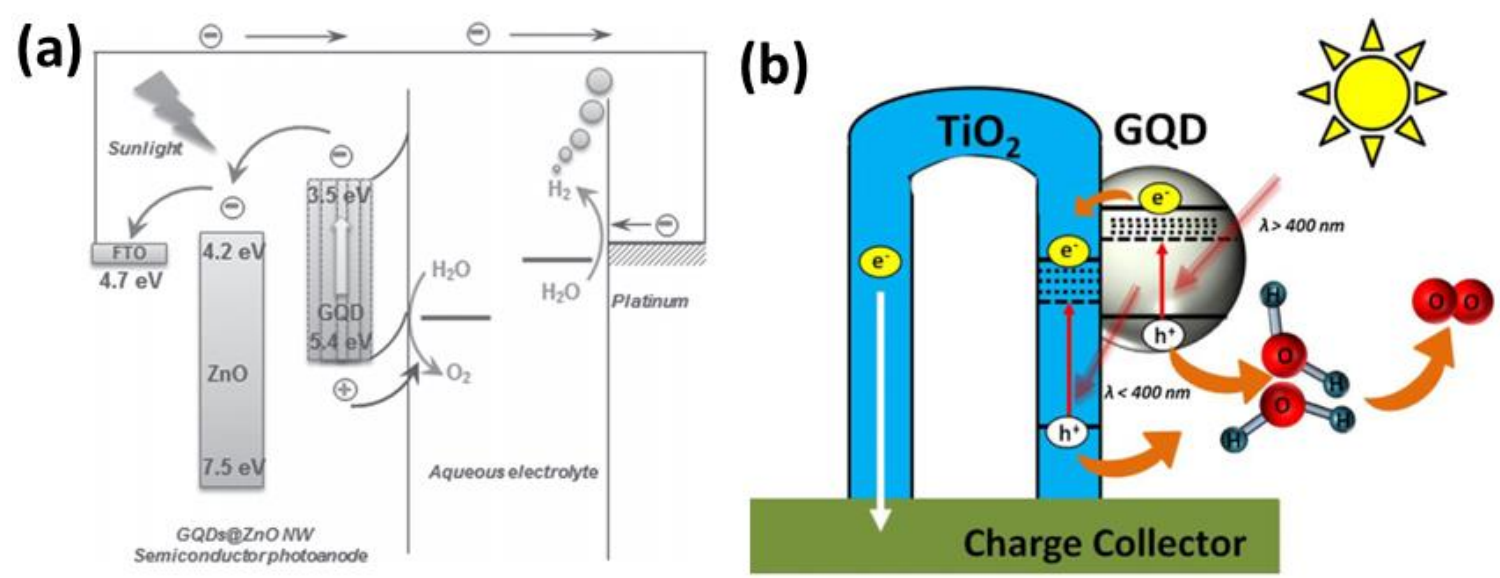

Figure 2-17. (a) Scheme of photoelectrochemical water splitting performance of GQDs@ZnO NWs.(152) Reproduced in permission from Ref.(152). Copyright 2013 Wiley-VCH. (b) Photoexcitation charge carriers generated at GQDs/TiO interfaces.(155) Reproduced in permission from Ref.(155). Copyright 2016 Elsevier Publishing Group.

\subsubsection{Degradation}

GQDs sensitized $\mathrm{TiO}_{2}$ nanotube fabricated by a controllable electrophoretic approach shows high visible-light catalytic performance, long-term durability, and environmental friendliness, superior to CdS and CdSe QDs-based photocatalysts.(156) Gupta et al. synthesized GQDs infilled $\mathrm{TiO}_{2}$ nanotube arrays hybrid, which shows highly-efficient photocatalytic activity in UV-light irradiated MB degradation. It is ascribed to the broad absorption in the ultraviolet wavelength region, and fast photogenerated charge separation via the transfer of photoexcited electrons from $\mathrm{TiO}_{2}$ to GQDs, and the strong adsorption capacity of GQDs to MB molecules.(157) GQD/ZnO NWs fabricated by Ebrahimi et al. displayed a considerable enhancement on the photocatalytic degradation of $\mathrm{MB}$ under solar irradiation with a direct Zscheme mechanism. They also show the optimized GQDs $(0.4 \%) / \mathrm{ZnO}$ NWs possess highest photoactivity with about 3-fold enhancement as compared to pure $\mathrm{ZnO}$ NWs.(158) 
GQDs@ $\mathrm{SnO}_{2}$ nanocompounds prepared by a facile process with pyrolyzing of citric acid (CA) show much enhanced photodegradation activity of MB than pure $\mathrm{SnO}_{2}$ nanorods (Figure 2-18a,b), which is ascribed to the realization of visible-light response and high charge-separation efficiency.(159) $\mathrm{GQDs} / \mathrm{AgVO}_{3}$ heterojunctions fabricated by Lei et al. show considerable enhancement on the photocatalytic performance for the visible-light irradiated degradation of pharmaceutical pollutant, such as Ibuprofen (IBP), as compared to bare $\mathrm{AgVO}_{3}$ nanoribbons. The photocatalytic activity of $3 \mathrm{wt} \%-\mathrm{GQD} / \mathrm{AgVO}_{3}$ heterojunctions has the optimized activity and excellent repeatability.(160) The hybrid heterostructures of GQD decorated $\mathrm{BiVO}_{4}$ nanoribbons fabricated by Tang et al show enhanced carbamazepine (CBZ) degradation, and $\bullet \mathrm{OH}$ was the predominant species in the $\mathrm{CBZ}$ photocatalytic degradation process (Figure 2-18c,d).(161)

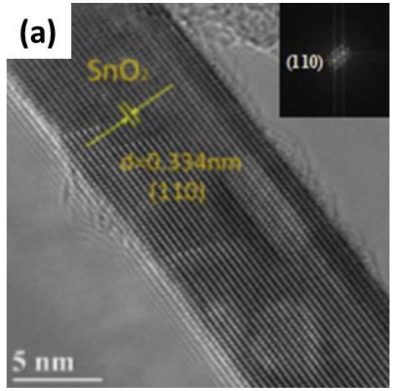

(b)
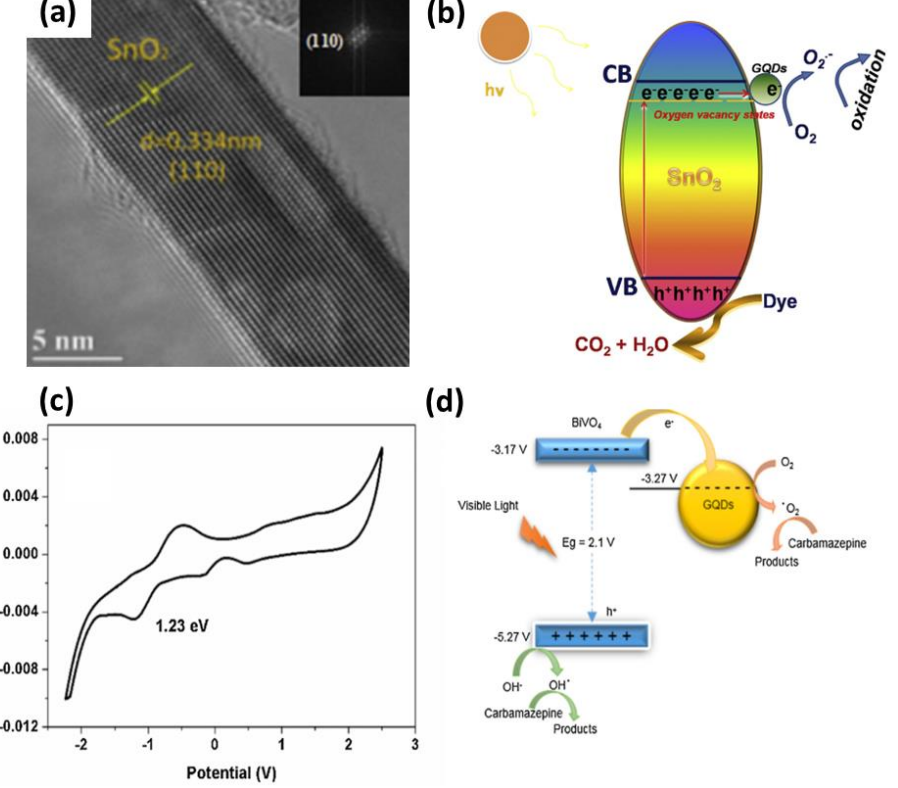

(d)

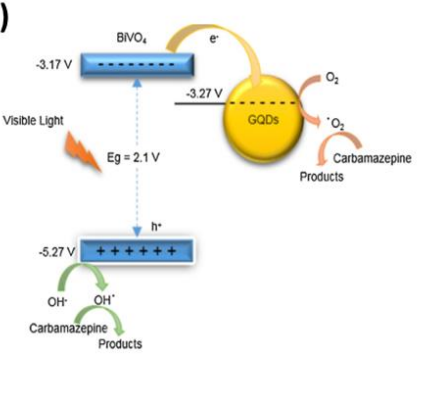

Figure 2-18. (a) HRTEM image of GQDs@ $\mathrm{SnO}_{2}$-VOs samples. (b) Scheme of the mechanism of photocatalytic activity of GQDs@SnO Reproduced in permission from Ref.(159). Copyright 2016 Elsevier Publishing Group. 


\subsubsection{GQDs-based 2 D materials}

\subsubsection{HER}

GQDs doped $\mathrm{MoS}_{2}$ nanosheets through a hydrothermal treatment possess defectrich features in both basal plane and edge plane, showing excellent enhanced electrocatalytic HER performance, such as low onset overpotential, high efficiency, and good catalytic stability.(162) GQDs- $\mathrm{Cu}_{2} \mathrm{O}$ was introduced to a bipolar membrane (BPM) interlayer, leading to $\mathrm{GQDs}-\mathrm{Cu}_{2} \mathrm{O} / \mathrm{BPM}$ composite as an excellent separator to prevent the crossover of $\mathrm{H}_{2}$ and $\mathrm{O}_{2}$.(163) $\mathrm{Fe}_{2} \mathrm{O}_{3}$-GQDs/NF-TiO ${ }_{2}$ composite film was prepared by loading $\alpha-\mathrm{Fe}_{2} \mathrm{O}_{3}$ and GQDs onto pyramid-shaped $\mathrm{N}$ and $\mathrm{F}$ co-doped $\mathrm{TiO}_{2}\left(\mathrm{NF}-\mathrm{TiO}_{2}\right)$. The composite film show far better PEC performance for $\mathrm{Cr}(\mathrm{VI})$ reduction and catalytic stability by utilizing $\mathrm{Fe}_{2} \mathrm{O}_{3}-\mathrm{GQDs} / \mathrm{NF}-\mathrm{TiO}_{2}$ as anode and a titanium sheet as cathode (Figure 2-19), which is attributed to the synergistic effect of extraordinary electron accelerators (GQDs) and visible-light active components ( $\alpha$ $\mathrm{Fe}_{2} \mathrm{O}_{3}$ and $\left.\mathrm{NF}-\mathrm{TiO}_{2}\right) .(164)$
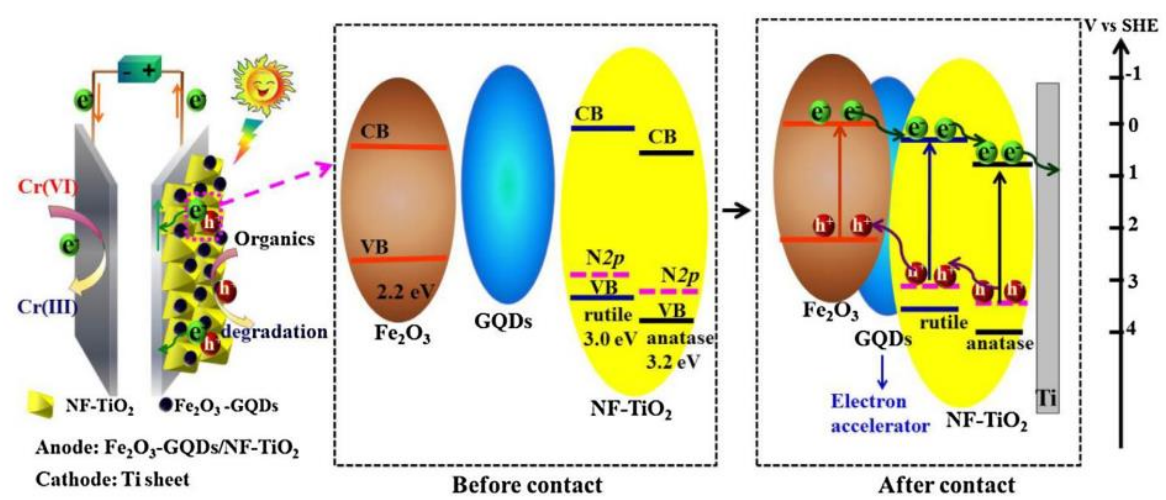

Figure 2-19. Scheme of the PEC reduction of $\mathrm{Cr}(\mathrm{VI})$ and the change of photogenerated charge on $\mathrm{Fe}_{2} \mathrm{O}_{3}-\mathrm{GQD} / \mathrm{NF}-\mathrm{TiO}_{2}$ composite.(164) Reproduced in permission from Ref.(164). Copyright 2017 Elsevier Publishing Group.

\subsubsection{ORR}

Nitrogen-doped GQDs/graphene hybrid materials with the extraordinary structural properties of N-GQDs, such as abundant pyridine-N, crystal structure, and 
rich-edges, show excellent electrochemical stability and resistance to the crossover of methanol for ORR application.(165) Nitrogen-doped GQDs/reduced graphene oxide (rGO) prepared by utilizing dopamine (DA) as the nitrogen-rich source and rGO as the electrocatalyst support (Figure 2-20a) displayed comparable ORR catalytic performance to that of the commercial $\mathrm{Pt} / \mathrm{C}$ catalyst as well as better durability and higher tolerance to the crossover effect of methanol.(166) Boron- and nitrogencodoped GQDs/graphene hybrid nanoplatelets (Figure 2-20b,c), with abundant edges and doping sites, large surface area, and high electrical conductivity, shows excellent ORR performance with $\sim 15 \mathrm{mV}$ more positive onset potential and similar current density when compared to commercial Pt/C in alkaline.(167)

N,S-rGO/GQDs composites fabricated by a solvothermal method assisted by microwave treatment displayed extraordinary electrocatalytic performance and promising prospects as cheap, metal-free electrochemical catalysts with a long-term stability for ORR activity to replace commercial Pt/C catalysts.(168) 
(a)

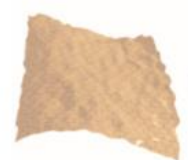

Go

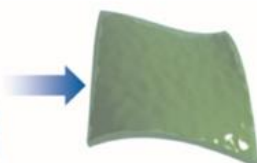

GO/PDA

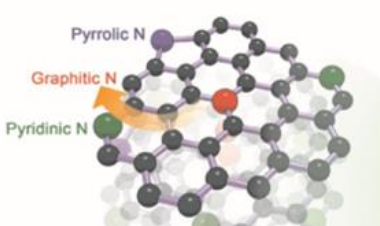

ure 1. The preparation procedure for the $\mathrm{N}-\mathrm{GQD} / \mathrm{rGO}$ catalyst

(b)

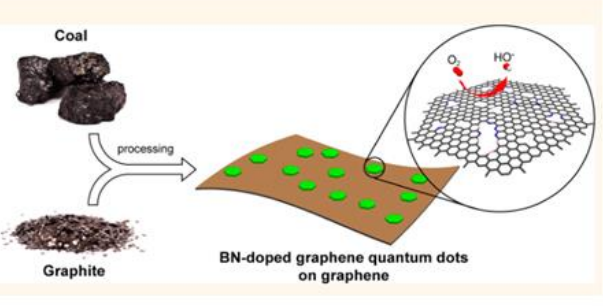

(d)

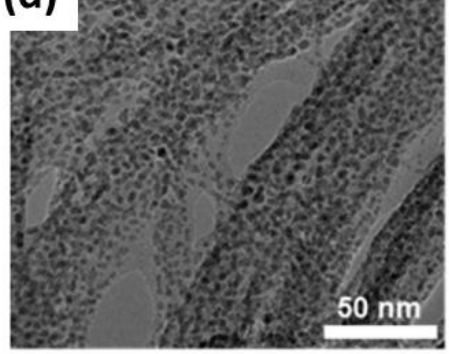

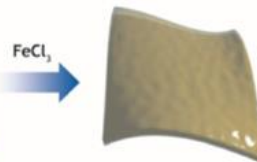

Fe-PDAGO

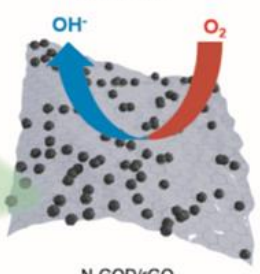

N-GQD/rGO

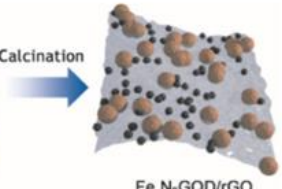

Fe,N-GQD/rGO

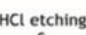

calcination

Calcination

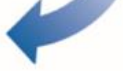

(c)
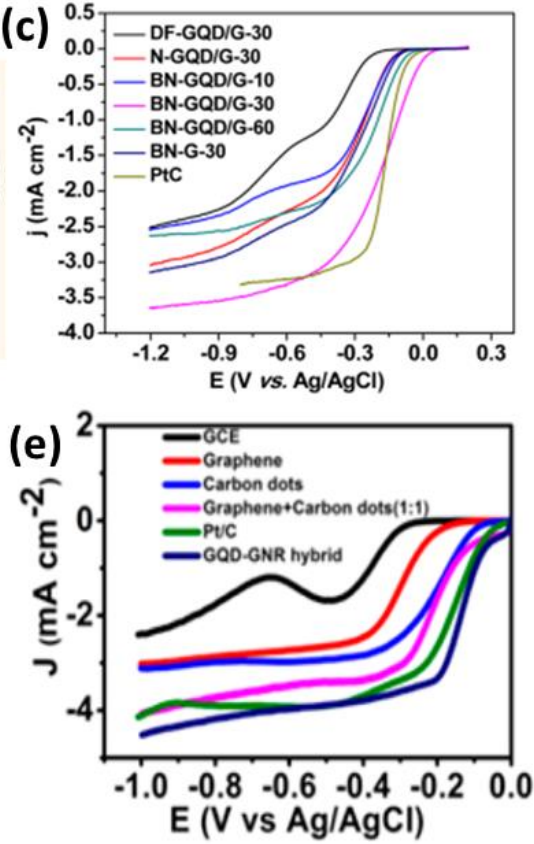

Figure 2-20. (a) Scheme of the preparation procedure for N-GQDs/rGO catalyst.(166) Reproduced in permission from Ref.(166). Copyright 2016 WileyVCH. (b) Scheme of the fabrication procedure for BN-GQDs/graphene nanocomposite, and (c) Linear sweep voltammograms (LSV) of ORR on different samples.(167) Reproduced in permission from Ref.(167). Copyright 2014 American Chemical Society. (d) TEM image of the as-prepared hierarchically structured GQDs-GNR hybrid, and (e) LSV of ORR on various samples at a disk rotating rate of $1600 \mathrm{rpm}$.(169) Reproduced in permission from Ref.(169). Copyright 2015 American Chemical Society.

GQDs/graphene nanoribbons (GNRs) (Figure 2-20d) developed by Jin et al displayed superior properties for ORR with an extraordinary electrocatalytic performance and good stability and selectivity in alkaline media as compared to the other C-based ORR catalysts (Figure 2-20e).(169) Si-GQDs electrodes fabricated by 
a mixture of GQDs and Si nanosheets show high catalytic activity for ORR in alkaline media, resulting from the high activity of pyridinic and pyrrolic nitrogen containing GQDs and efficient mass transport and electron transfer provided by $\mathrm{Si}$ nanosheets.(170)

\subsubsection{Antibacterial and antifouling}

GQDs-incorporated reverse osmosis membranes fabricated by Song et al displayed improved antifouling and chloride resistance properties with a stable water flux and a $\mathrm{NaCl}$ rejection of $98.8 \%$ at 16 bar, as well as a long-term durability over $120 \mathrm{~h}$ of RO testing.(171) Thin film nanocomposites (TFN) membranes comprising GOQDs dispersed within a tannic acid (TA) film were fabricated by an interfacial polymerization reaction for low-pressure nanofiltration (NF) (Figure 2-21a).(47) The resultant TA/GOQDs TFM membranes showed a high permeate water flux and dye rejections with good antifouling properties, which was ascribed to the favorable changes in membrane hydrophilicity, $\zeta$-potential, and surface roughness.

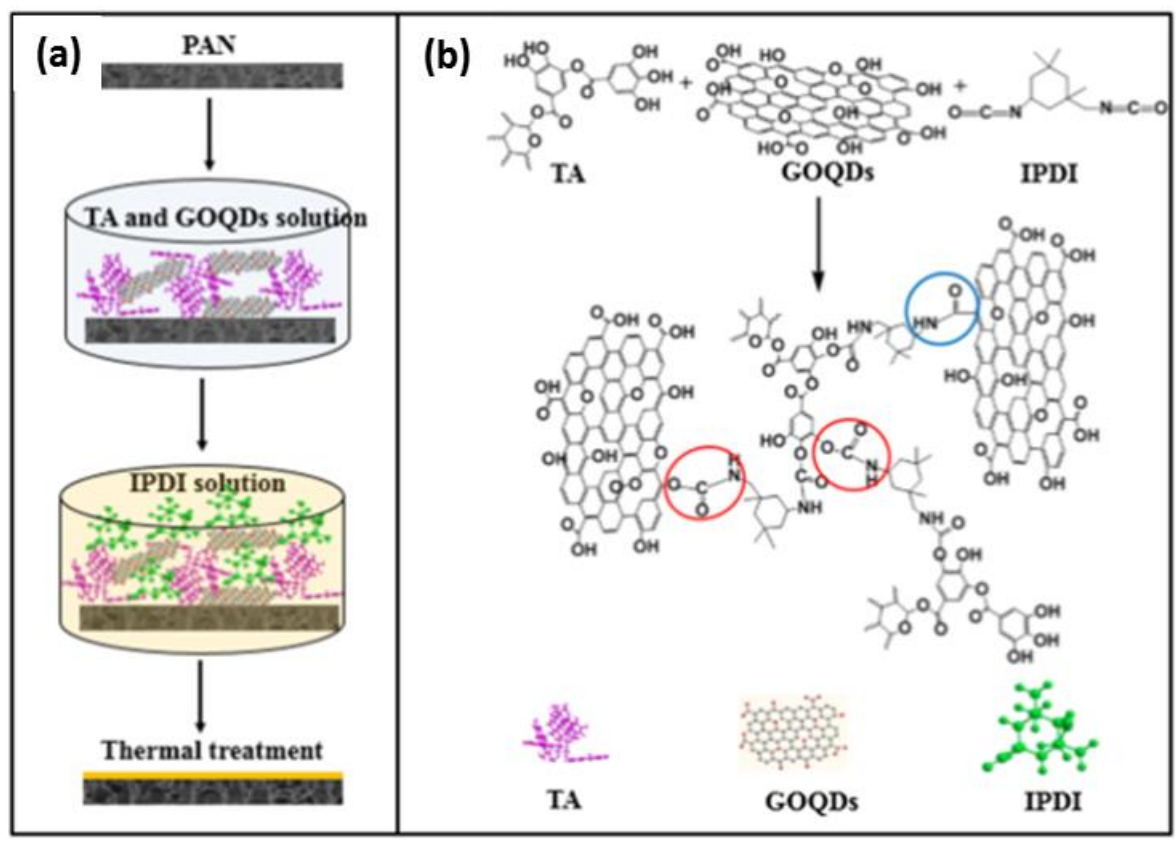

Figure 2-21. (a) Scheme of fabrication process of TA/GOQDs TFN membrane. (b) Schematic illustration of reaction mechanism among TA, GOQDs and 
IPDI.(47) Reproduced in permission from Ref.(47). Copyright 2017 American Chemical Society.

\subsubsection{Sensor, PL shift and Degradation}

$\mathrm{Pd} / \mathrm{GQDs} / \mathrm{WO}_{3}$ thin films presented significantly enhanced sensitivity towards $\mathrm{H}_{2}$ as lowering the working temperature to 120 with short response and recovery times. It is because they used GQDs to develop an electron conductive network and shorten the current transport paths.(172)

GQDs/MoS 2 heterostructures fabricated by depositing GQDs solutions onto the $\mathrm{MoS}_{2}$ monolayer provide an optical n-type doping mechanism and an effective modulation of $\mathrm{MoS}_{2}$ based on $0 \mathrm{D} / 2 \mathrm{D}$ heterostructures.(173) The doped carrier density $\left(6.5 \times 10^{13} \mathrm{~cm}^{-2}\right)$ demonstrated the effective n-type doping treatment in the interface between GQDs and $\mathrm{MoS}_{2}$.

N, S co-doped GQDs-rGO-TiO 2 nanotubes (NTs) composites fabricated by Tian et al(174) exhibit high photodegradation efficiency for visible-light irradiated degradation of methyl orange (MO).

\subsubsection{GQDs-based 3 D materials}

\subsubsection{Photocatalytic degradation}

GQD-decorated carbon aerogels (GQDs/CA) composite fabricated by Wang et al was used as a catalyst electrode for the electrichemical reduction of $\mathrm{I}^{3-}$ to $\mathrm{I}^{-}$, which is of extreme importance for the electrolyte regeneration reaction in dye-sensitized solar cells (DSSCs).(175) The cyclic voltammograms and Tafel data of the GQDs/CA electrode shows its catalytic efficiency can be comparable to that of Pt electrodes, but surpass Pt electrode in terms of long-term stability.

Sub-3 nm GQDs-modified mesoporous $\mathrm{Bi}_{2} \mathrm{MoO}_{6}$ (GQDs-BM) (Figure 22a,b) displayed good electrons-holes separation efficiency and broad spectrum 
photocatalytic activity under UV, visible and NIR light irradiation, and effectively enhanced efficiency of photo-excited electrons $(\cdot \mathrm{OH})$, thus strongly enhancing the photo-degradation activities.(176)
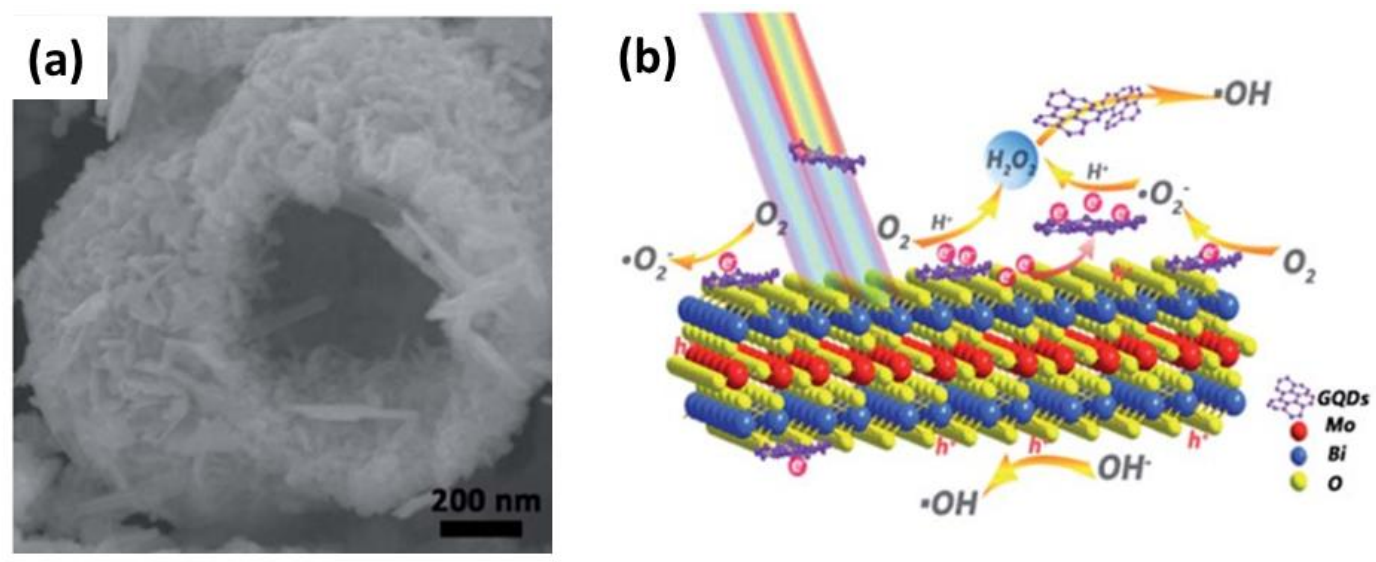

(c)

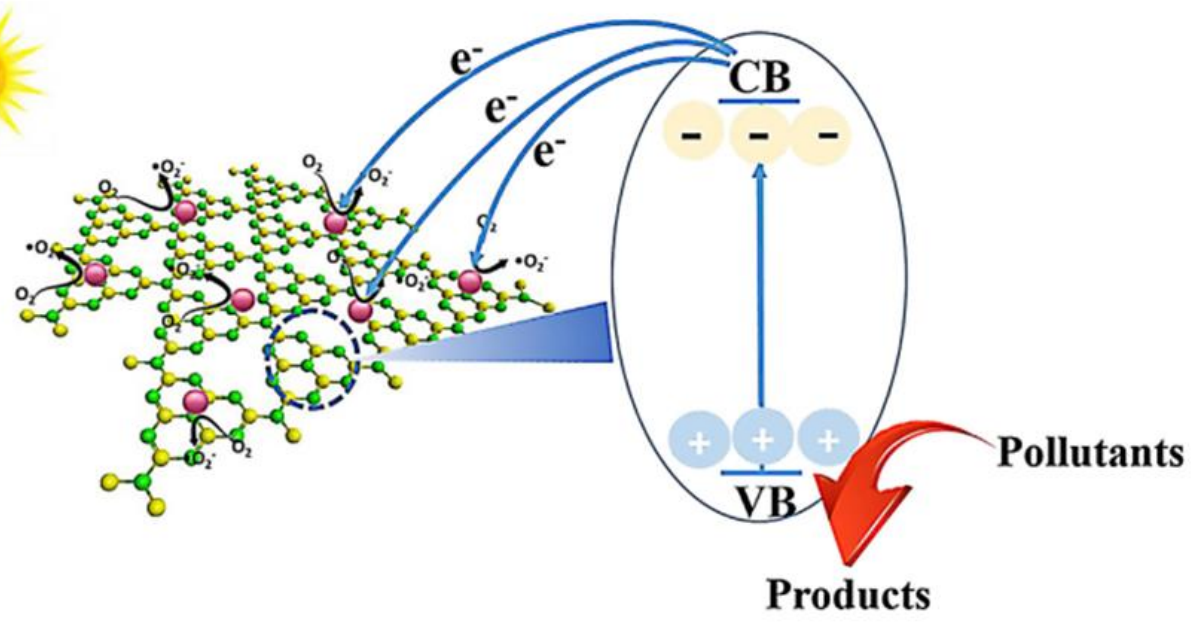

Figure 2-22. (a) SEM image and (b) Scheme of the photocatalysis mechanism of prepared GQDs-BM hybrid material.(176) Reproduced in permission from Ref.(176). Copyright 2016 Royal Chemical Society. (c) Scheme for the photocatalytic mechanism of GQDs/mpg- $\mathrm{C}_{3} \mathrm{~N}_{4}$ composite.(177) Reproduced in permission from Ref.(177). Copyright 2017 Elsevier Publishing Group.

GQDs modified mesoporous graphitic carbon nitride (GQDs/mpg- $\mathrm{C}_{3} \mathrm{~N}_{4}$ ) composites fabricated via electrostatic interactions showed high photocatalytic degradation activity toward $\mathrm{RhB}$, as well as removal of tetracycline hydrochloride (TC) pollutants under visible light irradiation. The GQDs/mpg- $\mathrm{C}_{3} \mathrm{~N}_{4}$ can generate a 
large amount of $\mathrm{O}_{2}{ }^{-}$species and a small fraction of holes (Figure 2-22c) for photocatalytic degradation.(177)

\subsubsection{HER}

GQDs modified coral-shaped $\mathrm{MoS}_{2}\left(\mathrm{MoS}_{2} @ \mathrm{GQDs}\right)$ with a large amount of exposed edges and a zero bandgap shows outstanding HER catalytic activities, including a smaller onset overpotential, lower Tafel slope, low applied potential at a current density $\left(10 \mathrm{~mA} \mathrm{~cm}^{-2}\right)$, and excellent cycling stability, attributed to the abundant $\mathrm{MoS}_{2}$ active edge sites and the enhanced charge transfer.(178) Nitrogendoped GQDs- $\mathrm{ZnNbO}_{6} / \mathrm{g}-\mathrm{C}_{3} \mathrm{~N}_{4}$ heterostructures fabricated by Yan et al showed highly efficient photocatalytic performance for water splitting under visible light illustration, with a high hydrogen-evolution rate of $340.9 \mu \mathrm{mol} \mathrm{h} \mathrm{h}^{-1} \mathrm{~g}^{-1}$.(135)

\subsubsection{ORR}

N-GQDs-decorated $\mathrm{MoS}_{2}$-rGO with a 3D framework fabricated by Vinoth et al was demonstrated as an efficient electrocatalyst toward ORR under alkaline conditions with a good stability and an outstanding methanol tolerance property at a high concentration of methanol.(179) 3D polyoxometalate (NaPWO) functionalized GQDs with mono-metallic and bi-metallic nanoparticles (NPs) exhibit relatively high peak current for methanol oxidation.(180)

In summary, the performances of GQDs-based materials were provided as following (Table 2.2).

Table 2.2 The performances of GQDs based materials

\begin{tabular}{|l|l|l|l|}
\hline \multicolumn{2}{|l|}{ Samples } & \multicolumn{2}{c|}{ Performances } \\
\hline & $\begin{array}{l}\text { Carboxyl } \\
\text { functionalized }\end{array}$ & \multirow{2}{*}{$\begin{array}{l}\text { Thermal } \\
\text { GQDs (116) }\end{array}$} & An acidic catalyst for the coupling reaction \\
& $\begin{array}{l}\text { Carboxyl and nitrite } \\
\text { functionalized GQD }\end{array}$ & & $\begin{array}{l}\text { An nitrosonium ion source and reusable } \\
\text { catalyst for rapid diazotization }\end{array}$ \\
\cline { 3 - 4 }
\end{tabular}




\begin{tabular}{|c|c|c|c|}
\hline \multirow{9}{*}{ GQDs } & $(117)$ & & \\
\hline & $\begin{array}{l}\text { Nitrogen-doped } \\
\text { graphene oxide } \\
\text { quantum dots } \\
\text { (NGO-QDs) (16) }\end{array}$ & Photocatalysts & $\begin{array}{l}\mathrm{H}_{2} \text { generation with } 12.8 \% \text { of quantum yield } \\
\text { under } 420 \mathrm{~nm} \text { light irradiation }\end{array}$ \\
\hline & $\begin{array}{l}\text { Nitrogen-doped } \\
\text { GQDs } \\
(98,120,121), \\
\text { B-N codoped } \\
\text { GQDs }(122) \\
\end{array}$ & Electrocatalysts & $\begin{array}{l}\text { an efficient electrocatalytic performance for } \\
\text { the ORR }\end{array}$ \\
\hline & $\begin{array}{l}\text { GQDs prepared } \\
\text { form C60 cage } \\
(123)\end{array}$ & \multirow[t]{2}{*}{ Antibacteria } & $\begin{array}{l}\text { Kills Staphylococcus aureus, but not other } \\
\text { bacterial }\end{array}$ \\
\hline & $\begin{array}{l}\text { GQDs fabricated } \\
\text { from (MWCNTs) } \\
(124)\end{array}$ & & $\begin{array}{l}\text { Selective toxicity towards bacterial cells but } \\
\text { low toxicity to mammalian cells }\end{array}$ \\
\hline & \multirow[t]{3}{*}{$\begin{array}{l}\text { N-GQDs }(125, \\
126,127)\end{array}$} & \multirow{4}{*}{ Sensor } & $\begin{array}{l}\text { An electrochemical detection of } 2,4,6- \\
\text { trinitrotoluene }\end{array}$ \\
\hline & & & $\begin{array}{l}\text { Rapid and sensitive determination of metal } \\
\text { ions }\left(\mathrm{Ca}^{2+}\right)\end{array}$ \\
\hline & & & Electrochemiluminescence system \\
\hline & $\begin{array}{l}\text { N,S-codoped } \\
\text { GQDs }(128)\end{array}$ & & $\begin{array}{l}\text { A new fluorescent probe for } \mathrm{Au}^{3+} \text { ions in real } \\
\text { aqueous media }\end{array}$ \\
\hline \multirow{8}{*}{$\begin{array}{l}\text { GQDs- } \\
\text { based } \\
0 \mathrm{D} \\
\text { materials }\end{array}$} & $\mathrm{GQDs} / \mathrm{Fe}_{3} \mathrm{O}_{4}(130)$ & \multirow{4}{*}{$\begin{array}{l}\text { Thermal } \\
\text { catalysts }\end{array}$} & $\begin{array}{l}\text { An effective catalyst for the removal of } \\
\text { phenolic compounds from solutions }\end{array}$ \\
\hline & $\mathrm{Au} / \mathrm{GQDs}(131)$ & & $\begin{array}{l}\text { A catalyst to oxidize veratryl alcohol to } \\
\text { veratric acid or veratryl aldehyde }\end{array}$ \\
\hline & Ag@GQDs (132) & & $\begin{array}{l}\text { Catalytic activity for 4-nitrophenol (4-NP) } \\
\text { reduction }\end{array}$ \\
\hline & $\begin{array}{l}\text { Pd supported on } \\
\text { N-GQDs (133) }\end{array}$ & & $\begin{array}{l}\text { A catalyst in the reduction reaction of } \\
\text { nitroaromatics }\end{array}$ \\
\hline & $\begin{array}{l}\text { N-GQDs/g- } \mathrm{C}_{3} \mathrm{~N}_{4} \\
(134), \\
\text { NGQDs- } \\
\mathrm{ZnNb}_{2} \mathrm{O}_{6} / \mathrm{g}-\mathrm{C}_{3} \mathrm{~N}_{4} \\
(135) \\
\mathrm{S}, \mathrm{N} \text { co-doped } \\
\text { GQDs- } \mathrm{C}_{3} \mathrm{~N}_{4}(124)\end{array}$ & \multirow[t]{4}{*}{$\begin{array}{l}\text { Photocatalysts } \\
\text { (HER, } \\
\text { Degradation) }\end{array}$} & $\begin{array}{l}\mathrm{H}_{2} \text { evolution from water splitting under visible } \\
\text { light irradiation; } \\
\text { Photocatalytic activity for degrading RhB }\end{array}$ \\
\hline & $\begin{array}{l}\text { GQDs/TiO } \\
(30,136,137,144) \\
\end{array}$ & & $\begin{array}{l}\text { High hydrogen evolution rate; photo- } \\
\text { degradation of MB }\end{array}$ \\
\hline & $\begin{array}{l}\text { GQDs decorated } \\
\mathrm{ZnO} \text { and } \mathrm{Cu} \\
\text { composites }(146)\end{array}$ & & UV light-irradiated Photodegradation of RhB \\
\hline & $\begin{array}{l}\text { S,N co-doped } \\
\text { GQDs/TiO } 2 \text { (145); } \\
\mathrm{L}-\mathrm{ZnO} / \mathrm{S}, \mathrm{N}-\mathrm{GQD} \\
(147) \\
\mathrm{Ag-GQDs-ZnO} \\
(148)\end{array}$ & & $\begin{array}{l}\text { Photodegradation of RhB under visible light } \\
\text { illumination }\end{array}$ \\
\hline
\end{tabular}




\begin{tabular}{|c|c|c|c|}
\hline & Pt/GQDs (138) & & A high positive onset potential $(+1.05 \mathrm{~V})$ for \\
\hline & $\begin{array}{l}\text { PtCu@GQDs } \\
\text { (139) }\end{array}$ & Electrocatalysts & $\begin{array}{l}\text { Enhanced catalytic activity in ORR with a two } \\
\text { times high mass activity }\end{array}$ \\
\hline & $\begin{array}{l}\text { Pd/GQDs (140), } \\
\text { Pd/N-GQDs (141) }\end{array}$ & & $\begin{array}{l}\text { Excellent electrocatalytic activity for ORR in } \\
\text { alkaline media }\end{array}$ \\
\hline & $\mathrm{Cu} / \mathrm{GQDs}(142)$ & & \\
\hline & $\begin{array}{l}\text { Sulfur-doped- } \mathrm{C}_{3} \mathrm{~N}_{4} \\
\text { modified GQDs } \\
(143)\end{array}$ & & Enhanced catalytic performance in the ORR \\
\hline & $\begin{array}{l}\text { Au-GQDs } \\
\text { modified glass } \\
\text { carbon electrode } \\
(149)\end{array}$ & Sensor & Catechol sensing \\
\hline & $\begin{array}{l}\text { Au NPs-N-GQDs } \\
(150)\end{array}$ & & Electrochemical detection of $\mathrm{H}_{2} \mathrm{O}_{2}$ \\
\hline & $\begin{array}{l}\text { Ag NPs-decorated } \\
\text { with GQDs (151) }\end{array}$ & & Detection of biothiols and $\mathrm{Ag}^{+}$ \\
\hline & $\begin{array}{l}\text { GQDs-ZnO NWs } \\
(152,15\end{array}$ & & Favourable photoelectrodes or PEC water \\
\hline $\begin{array}{l}\text { GQDs- } \\
\text { based } \\
1 \mathrm{D} \\
\text { materials }\end{array}$ & 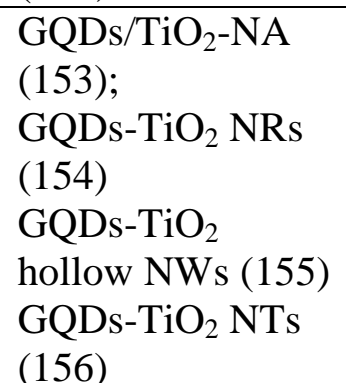 & $\begin{array}{c}\text { Photoelectrocata } \\
\text { lysts } \\
\text { (HER) }\end{array}$ & splitting; \\
\hline & $\begin{array}{l}\text { GQDs- } \mathrm{TiO}_{2} \text { NTs } \\
(157)\end{array}$ & & UV-light irradiated MB degradation \\
\hline & $\begin{array}{l}\text { GQD/ZnO NWs } \\
(158)\end{array}$ & Photocatalysts & $\begin{array}{l}\text { Photocatalytic degradation of MB under solar } \\
\text { irradiation }\end{array}$ \\
\hline & $\begin{array}{l}\text { GQDs@ } \mathrm{SnO}_{2} \\
(159)\end{array}$ & & $\begin{array}{l}\text { Enhanced visible-light irradiated } \\
\text { photodegradation activity of MB }\end{array}$ \\
\hline & $\begin{array}{l}\text { GQDs/AgVO } \\
(160)\end{array}$ & & $\begin{array}{l}\text { Visible-light irradiated degradation of } \\
\text { pharmaceutical pollutant (Ibuprofen) }\end{array}$ \\
\hline & $\mathrm{GQD} / \mathrm{BiVO}_{4}(161)$ & & Enhanced carbamazepine (CBZ) degradation \\
\hline & $\begin{array}{l}\text { GQDs doped } \mathrm{MoS}_{2} \\
\text { nanosheets (162) }\end{array}$ & & Enhanced electrocatalytic HER performance \\
\hline & $\begin{array}{l}\text { GQDs- } \mathrm{Cu}_{2} \mathrm{O} / \mathrm{BPM} \\
(163)\end{array}$ & $\begin{array}{l}\text { Electrocatalysts } \\
\text { (HER, ORR) }\end{array}$ & \\
\hline & $\begin{array}{l}\text { N-GQDs-GR } \\
(165,166)\end{array}$ & & Comparable ORR catalytic performance \\
\hline GQDs- & $\begin{array}{l}\text { B, N-codopped } \\
\text { GQDs/GR (167) }\end{array}$ & & \\
\hline $\begin{array}{l}\text { based } \\
2 \mathrm{D}\end{array}$ & $\begin{array}{l}\text { N,S-rGO/GQDs } \\
(168)\end{array}$ & & \\
\hline materials & GQDs/GNRs (169) & & \\
\hline & Si-GQDs (170) & & \\
\hline
\end{tabular}




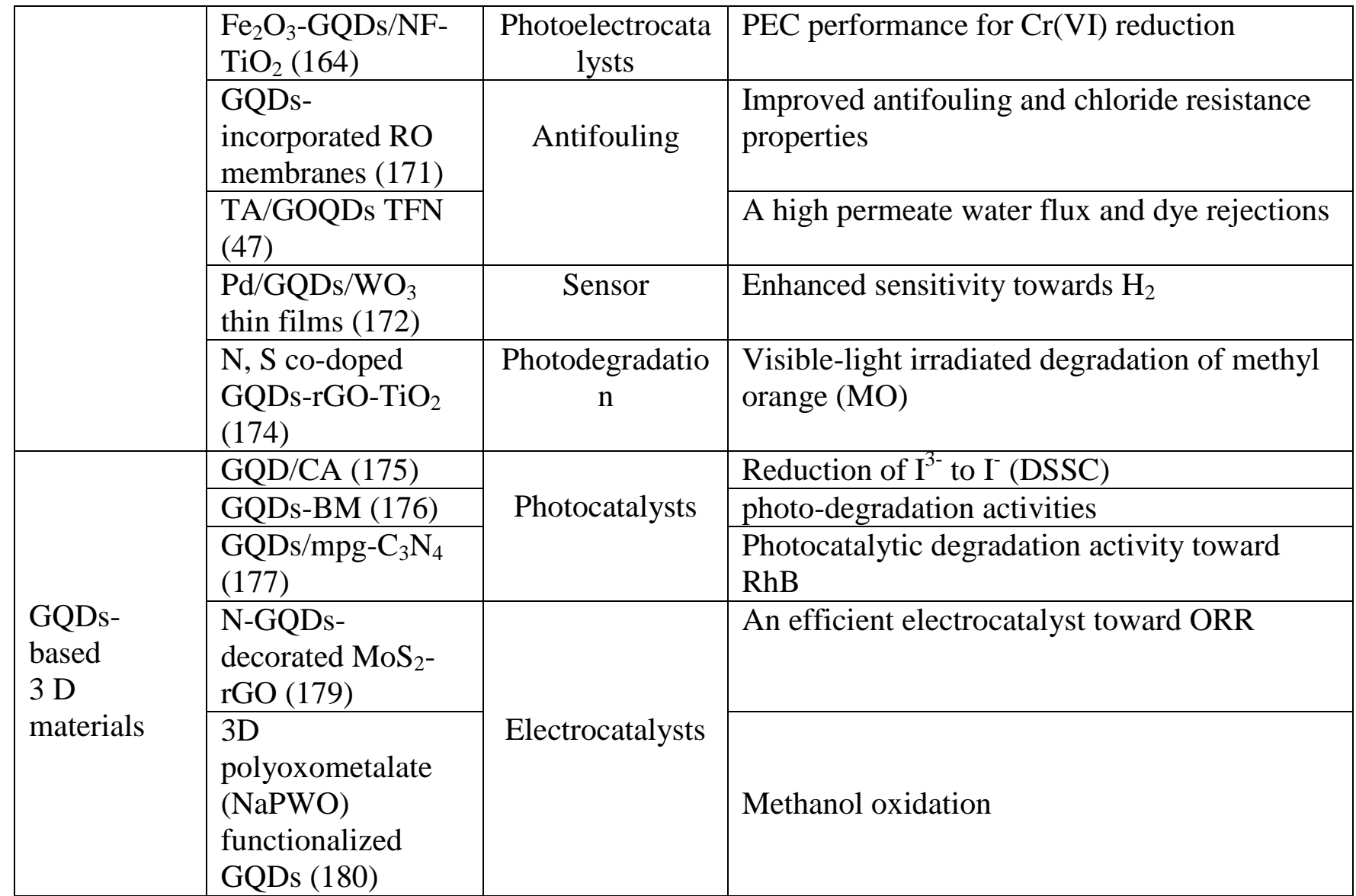




\section{Chapter 3 Unraveling Cooperative Synergy of}

\section{Carbon Nanomaterials and Metal Nanocrystals in}

\section{Multilayered Architectures towards Multifarious}

\section{Catalysis}

\subsection{Introduction}

Several works have reported the incorporation of metal NCs with GQDs for creating catalytically and electrocatalytically active nanomaterials for extensive applications. $(61,63,181,182)$ Despite the endeavors, there are still no investigations on progressive and controllable construction of metal/GQDs multilayers in which interfaces of metal NCs and GQDs are rationally designed and tuned. Additionally, GQDs can be recognized as one kind of carbon dots (CDs), which usually possess better crystallinity than its cousins.(100) GQDs have graphene lattices inside the dots, resembling the crystalline structure of single or few layered graphene. However, wide-spread potential applications of metal/GQDs multilayered nanoarchitectures have not yet been explored. Furthermore, conventional methods for constructing metal/GQDs nanocomposites such as simple mixing process are mainly centered on slurry systems with hard integration mode, which in fact cannot fully utilize and maximize the intrinsic properties of each active material, thereby leading to limited control over constituent components and failing to elucidate the structure-property relationship between the components in the nanocomposites. Alternatively, it should be emphasized that conventional approaches are relatively complicated and in some conditions involve delicate synthetic procedures and are not always environmentally friendly. Therefore, it is highly desirable to seek a facile, green, efficacious, easily 
accessible and scalable route to fabricate highly ordered metal/GQDs nanocomposites at ambient conditions with a fine structural control, such that the integration mode and interfaces of metal and GQDs can be finely modulated.

Layer-by-Layer (LbL) assembly is characteristic of sequential adsorption of materials with complementary functional groups employing electrostatic interactions, hydrogen bonding, or other intermolecular interaction. $(183,184)$ As one of the promising bottom-up techniques, LbL assembly demonstrates remarkable advantages in terms of its simplicity, accessibility, repeatability and versatility, which furnishes ideal nanoscale-level control over composition and structure of the assemblies with the choice of applicable materials and sequence of layering.(83-86) Recent years have witnessed a cornucopia toward the synthetic fabrication of multilayered architectures via LbL assembly; nonetheless, most of which depend heavily on utilizing polyelectrolytes (PEs) as glue mediums for assembling the components, with PEs ultimately removed via calcination. Apparently, it is of great significance to achieve direct LbL assembly by taking full advantage of the oppositely charged surfaces of building blocks without involving PEs, which would remarkably simplify the synthetic procedure and, more importantly, afford more intimate interfacial contact among assembly units. Inspired by this, considering the colloidal aqueous solutions of metal NCs and GQDs are quite stable and, thus, their surface charge properties can be finely tuned to trigger the spontaneous and "glueless" LbL assembly based on pronounced electrostatic attractive interaction, which is in favor of creating diverse multilayer nanostructures.

In this Chapter, a collection of GQDs enwrapped metal NCs (M/GQDs) $)_{n}(M=A u$, $\mathrm{Ag}, \mathrm{Pt}$ ) multilayers have been rationally designed and constructed by a direct LbL assembly strategy based on electrostatic interaction, in which negatively charged 
metal NCs and surface-modified positively charged GQDs acting as assembly units were tightly integrated at nanoscale level in an alternate stacked fashion. Owing to multiple cooperative synergies between the GQDs and NCs, these (M/GQDs)n multilayer thin films demonstrated highly efficient catalytic performances as well as versatility for different types reactions, including selective organic transformation of aromatic nitro alternatives, electrocatalytic methanol oxidation and solar lightirradiated photoelectrochemical (PEC) water splitting at ambient conditions. More significantly, catalytic performances of $(\mathrm{M} / \mathrm{GQDs})_{\mathrm{n}}$ multilayer thin films can be finely tuned and modulated by assembly cycle, assembly sequence, and type of metal NCs. Considering the versatile nature of LbL assembly coupled with GQDs and diverse types of metal NCs, the approach developed in the current work will offer conceptual new insights for rational design of metal-GQDs-based multilayered nanoarchitectures for a wide range of applications in advanced chemical-to-energy conversion.

\subsection{Experimental}

\subsubsection{Preparation of graphene oxide quantum dots (GQDs)}

\section{a. Preparation of GQDs}

Graphene oxide quantum dots (GQDs) were prepared with CX-72 carbon black via being refluxed in a concentrated nitric acid $\left(\mathrm{HNO}_{3}\right)$ solution. $(49,185)$ Typically, $0.4 \mathrm{~g}$ dried CX-72 carbon black was added to $6 \mathrm{~mol} \mathrm{~L}^{-1}$ nitric acid $(100 \mathrm{~mL})$ and refluxed for $24 \mathrm{~h}$ at $110^{\circ} \mathrm{C}$. After cooling to below $30^{\circ} \mathrm{C}$, the product was centrifuged (12000 rpm) for $10 \mathrm{~min}$ to achieve sediment and a supernatant. The resultant supernatant was treated at $200{ }^{\circ} \mathrm{C}$ to evaporate the nitric acid and water. After cooling to room temperature, a reddish-brown solid was acquired. Finally, GQDs aqueous solution was obtained by dissolving GQDs in DI $\mathrm{H}_{2} \mathrm{O}$ under 10 min sonication. 


\section{b. Preparation of surface charge-modified GQDs}

Positively charged GQDs (GQDs- $\left.\mathrm{NH}_{3}{ }^{+}\right)$or GR aqueous solution was synthesized via the 1-[3-(dimethylamino)propyl]-3-ethylcarbodiimide methyliodide and ethylene diamine (EDC)-mediated amine exchange reaction.(186) In specific, 0.5 g of 1-[3-(dimethylamino)propyl]-3-ethylcarbodiimide methyliodide was dissolved in $4 \mathrm{~mL}$ ethylenediamine, and the mixture was added into $40 \mathrm{ML}$ of negatively charged GQDs solution $(0.5 \mathrm{mg} / \mathrm{mL})$. The mixture can be fast stirred for $15 \mathrm{~h}$, subsequently dialyzed for 3 days by using a membrane (MWCO $=12000-14000 \mathrm{Da})$ to order to separate any residual chemicals.

\subsubsection{Synthesis of negatively charged citrate-stabilized metal nanocrystals}

\section{a. Preparation of Au NCs}

Citrate-stabilized gold nanocrystals (NCs) were synthesized by Dotzauer method.(187) Briefly, the glassware need to be washed with aqua region $\left(\mathrm{HCl} / \mathrm{HNO}_{3}=3: 1\right)$ and rinsed with DI water. $50 \mathrm{~mL}$ of aqueous $1 \mathrm{mM} \mathrm{HAuCl} \cdot 3 \mathrm{H}_{2} \mathrm{O}$ was heated to boiling whilst stirring. $38.8 \mathrm{mM}$ sodium citrate dihydrate $(5 \mathrm{~mL})$ was heated to boiling and then mixed with the Au solution. The mixture became dark and then burgundy, and then was heated with stirring for $10 \mathrm{~min}$, and was subsequently stirred without heating for an additional $15 \mathrm{~min}$.

\section{b. Preparation of Ag NCs}

Citrate-stabilized silver NCs were prepared according to Lee's approach.(188) Briefly, a sodium citrate solution $(40 \mathrm{mM}, 8 \mathrm{~mL})$ was added into $1 \mathrm{mM}$ aqueous $\mathrm{AgNO}_{3}$ solution $(100 \mathrm{~mL}) .112 \mathrm{mM} \mathrm{NaBH}$ solution $(2 \mathrm{~mL})$ was added dropwise under fast stirring, producing a yellowish brown silver hydrosol. The resultant silver 
hydrosol was then put in a refrigerator $\left(4^{\circ} \mathrm{C}\right)$ for one day to remove the residual $\mathrm{NaBH}_{4}$ for further use.

\section{c. Preparation of Pt NCs}

Citrate-stabilized platinum NCs was prepared by Elliott's method. Specifically, a trisodium citrate dehydrate solution $(2.8 \mathrm{mM}, 26 \mathrm{~mL})$ was mixed with hydrogen hexachloro-platinate solution $(0.4 \mathrm{mM}, 50 \mathrm{~mL})$. An amount of $12 \mathrm{mM} \mathrm{NaBH}$ solution $(5 \mathrm{~mL})$ was then added dropwise with fast stirring, resulting that the yellow solution turned dark brown. The resultant Pt solution was subsequently stirred for four hours and put in a refrigerator at $4{ }^{\circ} \mathrm{C}$.

\subsubsection{LbL assembly of $(\mathrm{M} / \mathrm{GQDs})_{\mathrm{n}}(\mathrm{M}=\mathrm{Au}, \mathrm{Ag}, \mathrm{Pt})$ multilayer composite thin} films

Fluorine-doped tin oxide (FTO) or silicon wafer substrates were thoroughly cleaned in a freshly prepared "piranha" solution $\left(3: 1\right.$ concentrated $98 \% \mathrm{H}_{2} \mathrm{SO}_{4} / 30 \%$ $\mathrm{H}_{2} \mathrm{O}_{2}$; Caution: piranha solution reacts violently with organic materials and should be handled with great care). Firstly, the substrate was immersed in polyethylenimine (PEI) solution $(1.0 \mathrm{mg} / \mathrm{mL}, \mathrm{pH}=7.23,0.5 \mathrm{M} \mathrm{NaCl})$ for around $10 \mathrm{~min}$, then rinsed 3 times with $\mathrm{DI} \mathrm{H}_{2} \mathrm{O}$, subsequently dried by using $\mathrm{N}_{2}$. After that, the substrate was put into as-prepared negatively charged metal aqueous solution for $10 \mathrm{~min}$ and then subjected to the same washing and drying treatments. Afterward, the substrate was dipped into surface charge modified GQDs (or GR) aqueous suspension $(1.0 \mathrm{mg} / \mathrm{mL}$, $\mathrm{pH}=7.0$ ) for 10 min, washed with $\mathrm{DI} \mathrm{H}_{2} \mathrm{O}$, and then dried with a gentle steam of $\mathrm{N}_{2}$, producing $(\mathrm{M} / \mathrm{GQDs})_{1}\left(\right.$ or $\left.(\mathrm{M} / \mathrm{GR})_{1}\right)$ multilayer thin film. The above treatment as one whole can be designated as one assembly cycle. Multilayer (M/GQDs $)_{n}\left(\right.$ or $\left.(M / G R)_{n}\right)$ composite thin films with varied assembly cycles were treated by alternate deposition 
of negatively charged metal NCs and positively charged GQDs (GR). For comparison, pure $(\mathrm{GQDs})_{\mathrm{n}},(\mathrm{GR})_{\mathrm{n}},(\text { metal } \mathrm{NCs})_{\mathrm{n}}$, multilayer thin films were prepared. For the preparation of pure $(\mathrm{GQDs})_{\mathrm{n}}$ (or $\left.(\mathrm{GR})_{\mathrm{n}}\right)$ multilayer thin films, the substrate was dipped into poly (sodium 4-styrenesulfonate) (PSS, $1.0 \mathrm{mg} / \mathrm{mL}, \mathrm{pH}=7.0$ ) aqueous solution for 10 min and rinsed 3 times with $\mathrm{DI}_{2} \mathrm{O}$, then dried by using $\mathrm{N}_{2}$. After that, the substrate was dipped in surface charge modified GQDs (or GR) aqueous solution for $10 \mathrm{~min}$ and then subjected to the same washing and drying treatments, producing $(\mathrm{GQDs})_{1}$ (or $\left.(\mathrm{GR})_{1}\right)$ multilayer thin film. The above treatment as one whole can be designated as one assembly cycle. Multilayer $(\mathrm{GQDs})_{\mathrm{n}}\left(\right.$ or $\left.(\mathrm{GR})_{\mathrm{n}}\right)$ thin films with varied assembly cycles were fabricated by alternate treatment of positively charged GQDs (or GR) and negatively charged PSS. Multilayer (metal NCs) n $_{\mathrm{n}}$ thin films with varied assembly cycles were fabricated by alternate treatment of positively charged PEI and negatively charged as-prepared metal NCs. Finally, the LbL-assembled multilayer composite films were calcined in an argon atmosphere furnace at $400{ }^{\circ} \mathrm{C}$ for $1 \mathrm{~h}$. The detailed assembly procedure was illustrated in Figure 3-1.

\subsubsection{Materials Characterizations}

Scanning electron microscopy (SEM) results were achieved with field emission scanning electron microscopy (JEOL, JSM-6700F). Transmission electron microscopy (TEM) images were achieved by utilizing a JEOL model JEM2010 (200 $\mathrm{kV}$ ). X-ray photo electron spectroscopy (XPS) characterization was collected through ESCALAB 250 photoelectron spectrometer (Thermo Fisher Scientific) at $2.4 \times 10^{-10}$ mbar. Atomic force microscopy (AFM) images were obtained by using MFP3D microscope, Asylum Research. UV-vis spectra were recorded on a Shimadzu UV2501 spectrophotometer in which $\mathrm{BaSO}_{4}$ was used a background ranging from 300 to $800 \mathrm{~nm}$. The fluorescence spectra were collected by a Fluoromax-4, Horiba 
Jobin Yvon Spectro fluorometer with a photon-counting detection system to detect fluorescence emission. Fourier Transform Infrared Spectroscopy (FTIR) was achieved by using a Digilab FTS 3100 instrument. The crystal phase of the samples can be recorded by X-ray diffraction (XRD, Bruker). The zeta potential measurement can be achieved by using dynamic light scattering analysis (Zeta PALS). Raman results were collected by using Raman spectroscopy (Renishaw) equipped with a 633 nm laser source.

\subsubsection{Selective catalytic reduction of aromatic nitro compounds}

As a representative example, the catalytic properties of $(\mathrm{M} / \mathrm{GQDs})_{\mathrm{n}}(\mathrm{M}=\mathrm{Au}, \mathrm{Ag}$, Pt; $n=1,5,10,15)$ multilayer thin films were evaluated by employing the reduction of aromatic nitro compounds to corresponding amino compounds by $\mathrm{NaBH}_{4}$ as a model reaction. In a typical reaction, $(\mathrm{M} / \mathrm{GQDs})_{\mathrm{n}}$ samples $\left(2 \mathrm{~cm}^{2}\right)$ was put in a quartz cuvette with $2 \mathrm{mM}(40 \mu \mathrm{L})$ aromatic nitro compound, $100 \mathrm{mM}(400 \mu \mathrm{L}) \mathrm{NaBH}_{4}$, and $2 \mathrm{~mL}$ DI $\mathrm{H}_{2} \mathrm{O}$. Subsequently, the solution was stirred for around $30 \mathrm{~min}$. The reaction samples were tested at specific time interval for UV-vis spectroscopy measurement.

\subsubsection{Electrochemical methanol oxidation}

Electrochemical experiments were conducted in a three-electrode quartz cell in which $\mathrm{Ag} / \mathrm{AgCl}$ electrode was used as a reference electrode and $\mathrm{Pt}$ sheet as counter electrode, and the $(\mathrm{M} / \mathrm{GQDs})_{\mathrm{n}}$ sample with the same active area $\left(15.9 \mathrm{~mm}^{2}\right)$, provided by a mask with a diameter of $4.5 \mathrm{~mm}$, were used as the working electrodes. linear sweep voltammetry (LSV) and Cyclic voltammetry (CV) were measured from 0.00 to $0.75 \mathrm{~V}$ in alkaline solution $(\mathrm{KOH}, 0.1 \mathrm{M})$ without or with $1.0 \mathrm{M} \mathrm{CH}_{3} \mathrm{OH}$ solution (scan rate $20 \mathrm{mV} / \mathrm{s}$ ). The measurements of electrochemical impedance spectroscopy 
(EIS) can be achieved in the frequency range $(100 \mathrm{kHz} \sim 100 \mathrm{mHz})$ under $\mathrm{AC}$ stimulus in amplitude $(10 \mathrm{mV})$.

\subsubsection{Photoelectrochemical (PEC) Measurement}

PEC measurements were carried out on an electrochemical workstation (Zennium, Zahner). The electrochemical setup is composed of conventional threeelectrodes, a quartz cell containing $20 \mathrm{~mL} \mathrm{Na}_{2} \mathrm{SO}_{4}(0.5 \mathrm{M})$ aqueous solution and a potentiostat. A phatinum plate $\left(10 \times 20 \mathrm{~mm}^{2}\right)$ was used as a counter electrode. The samples film $\left(10 \times 20 \mathrm{~mm}^{2}\right)$ were vertically immersed into electrolyte and irradiated with a $300 \mathrm{~W}$ xenon arc lamp (Newport) with a filter (AM 1.5). Monochromatic incident photo-to-electron conversion efficiency (IPCE) spectra were collected using three-electrode without bias, for which monochromatic light was provided by a 300 W xenon arc lamp (Newport) combined with a monochromator (Newport).

\subsection{Results and discussion}

3.3.1 Characterization of GQDs, metal nanocrystals and $(\mathrm{M} / \mathrm{GQDs})_{\mathrm{n}}(\mathrm{M}=\mathrm{Au}$, Ag, Pt) nanostructures

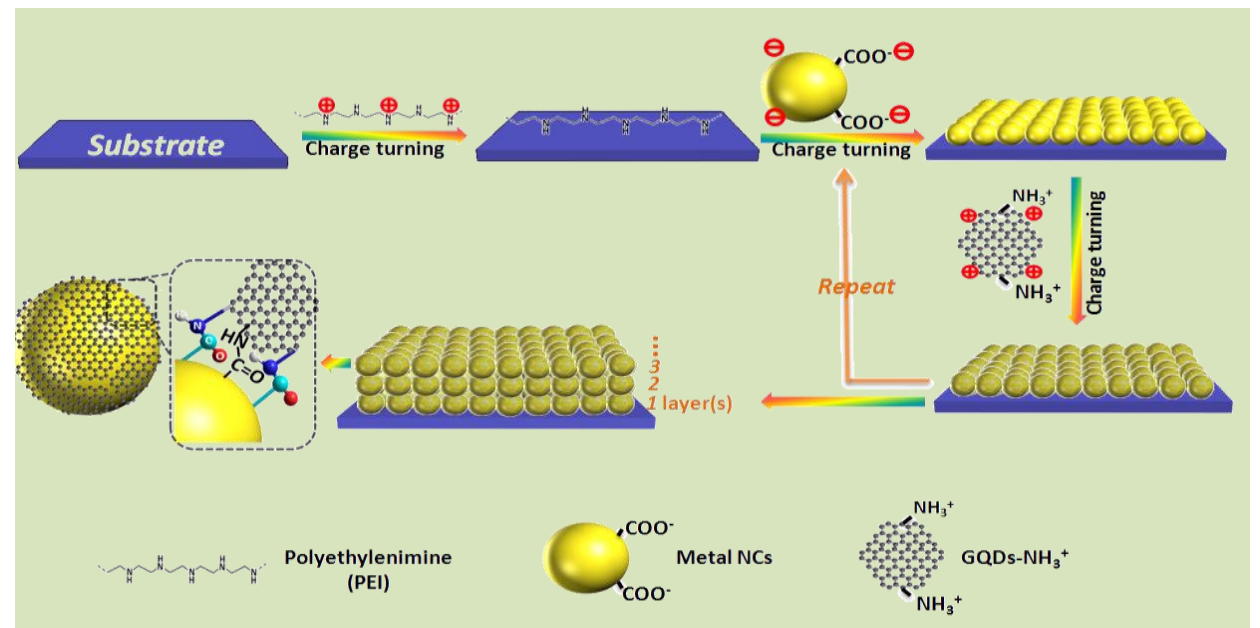

Figure 3-1. Scheme for LbL assembly of (M/GQDs) $(\mathrm{M}=\mathrm{Au}, \mathrm{Ag}, \mathrm{Pt}$; $\mathrm{n=1,5,10,15)}$ multilayer composite thin films. 
Figure 3-1 illustrates the flowchart for LbL assembly of (M/GQDs) $)_{n}$ multilayer thin films, in which intrinsically negatively charged metal $\mathrm{NCs}(\mathrm{Au}, \mathrm{Ag}, \mathrm{Pt})$ and surface-modified positively charged GQDs were used as the nanobuilding blocks. The colloidal metal NPs were capped with citrate ions which are able to deprotonate in aqueous phase, thus affording negatively charged surface for the metal NCs. As the oppositely charged counterpart in the LbL assembly, the surface of GQDs was judiciously converted from the intrinsically negatively charged to the positively charged by grafting a small molecules on the planar surface $\left(\mathrm{GQDs}-\mathrm{NH}_{3}{ }^{+}\right)$. In this regard, substantial electrostatic attractive interaction between the negatively charged metal NCs and positively charged GQDs provides a solid component for LbL assembly of a series of (M/GQDs) $)_{n}$ multilayer thin films.
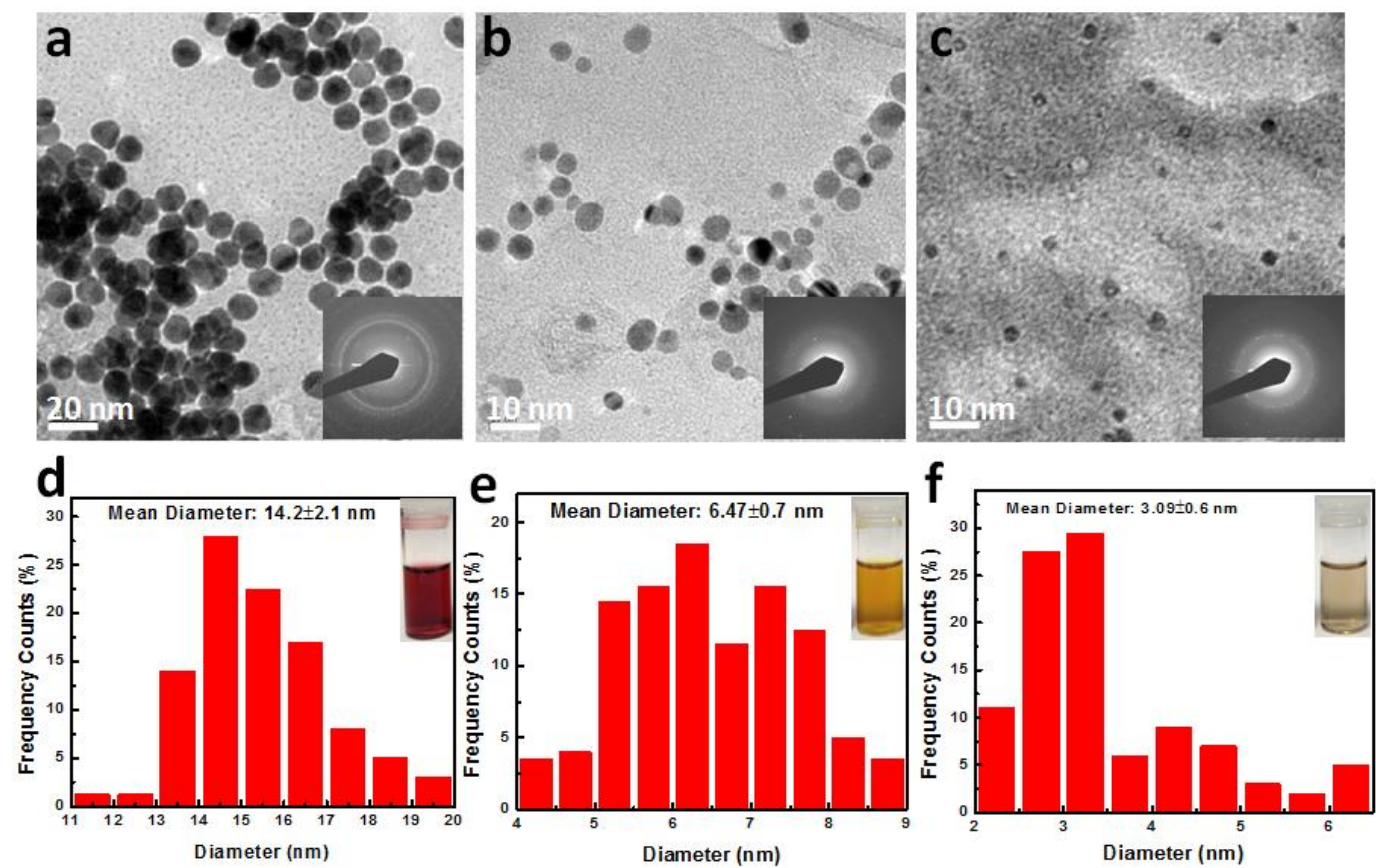

Figure 3-2. (a-c) TEM images of citrate-stabilized (a) Au, (b) Ag, and (c) Pt NCs (SAED patterns in the inset). (d-f) Mean diameter histogram of (d) Au, (e) Ag, (f) Pt NCs with corresponding digital photographs in the inset.

The LbL-assembly (M/GQDs) $)_{\mathrm{n}}$ multilayer composite films were firstly characterized. As shown in the TEM images (Figure 3-2), metal NCs including Au, 
$\mathrm{Ag}$, and Pt NCs demonstrated excellent monodispersivity (inset images, Figure 3-2) arising from substantial electrostatic repulsion and narrow size distribution with average diameters of $14.2 \pm 2.1,6.47 \pm 0.7$, and $3.09 \pm 0.6 \mathrm{~nm}$, respectively. Figure 32(a-c) show selective area electron diffraction (SAED) substantiate the polycrystalline feature of the metal NCs. Zeta potential results (Figure 3-4) strongly suggested that all these colloidal metal NCs are featured with pronounced negatively charge surfaces in a wide $\mathrm{pH}$ profile $(2 \sim 12)$, which is in favor of spontaneous electrostatic assembly with their positively-charged counterparts.
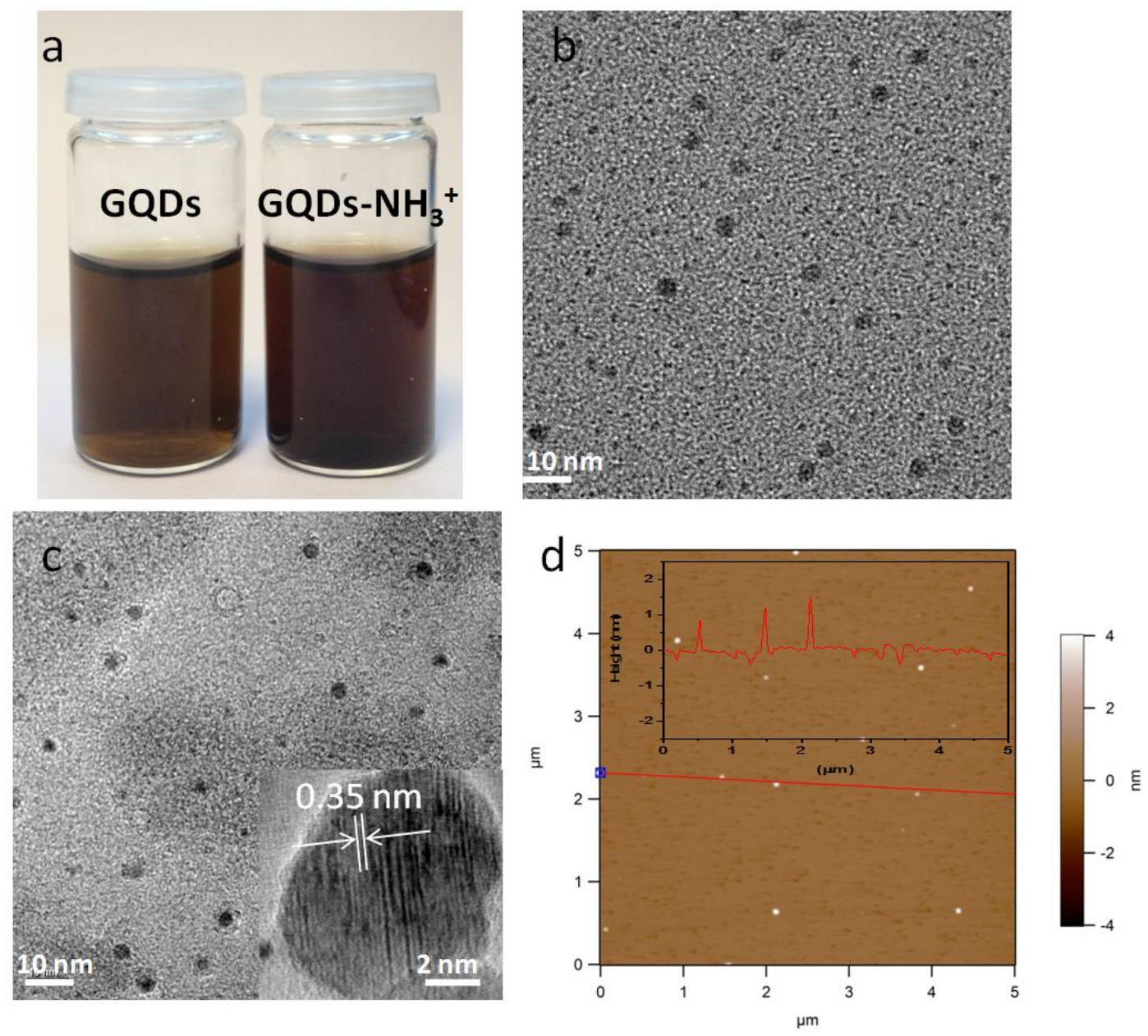

Figure 3-3. (a) Digital photographs of GQDs and GQDs- $\mathrm{NH}_{3}{ }^{+}$aqueous solution, (b) TEM image of GQDs. (c) TEM image of GQDs- $\mathrm{NH}_{3}{ }^{+}$(Inset: high-resolution image). (d) AFM image of GQDs- $\mathrm{NH}_{3}{ }^{+}$with the height profile along the red line. 
As displayed in Figure 3-3c, TEM image of GQDs- $\mathrm{NH}_{3}{ }^{+}$reflected uniform size of ca. $5.8 \mathrm{~nm}$, and the lattice-fringe spacing in the high-resolution (HR) TEM image in the inset was determined to be ca. $0.35 \mathrm{~nm}$, corresponding well to the (002) crystal planes of graphite. Atomic force microscope (AFM) image in Figure 3-3d manifested that height profile of GQDs- $\mathrm{NH}_{3}{ }^{+}$was around $0.7 \sim 1.2 \mathrm{~nm}$, suggesting that it consists of 1 2 graphene layers. Compared with pristine GQDs (Figure 3-3b), apparently, physical properties of GQDs were retained in GQDs- $\mathrm{NH}_{3}{ }^{+}$after modification. As unveiled by the zeta potential results in Figure 3-4, both GQD- $\mathrm{NH}_{3}{ }^{+}$ and $\mathrm{GO}-\mathrm{NH}_{3}{ }^{+}$aqueous solutions exhibited substantial positively charged surface, e.g., $55.2 \pm 1.6$ and $52.5 \pm 4.1 \mathrm{mV}$ in a $\mathrm{pH}$ profile ranging from 7 to 12 , respectively. Thus, stable surface charge properties of constituent ingredients for LbL assembly can be rationally tuned via altering the $\mathrm{pH}$ values of metal NCs and GQDs aqueous solutions.(189)

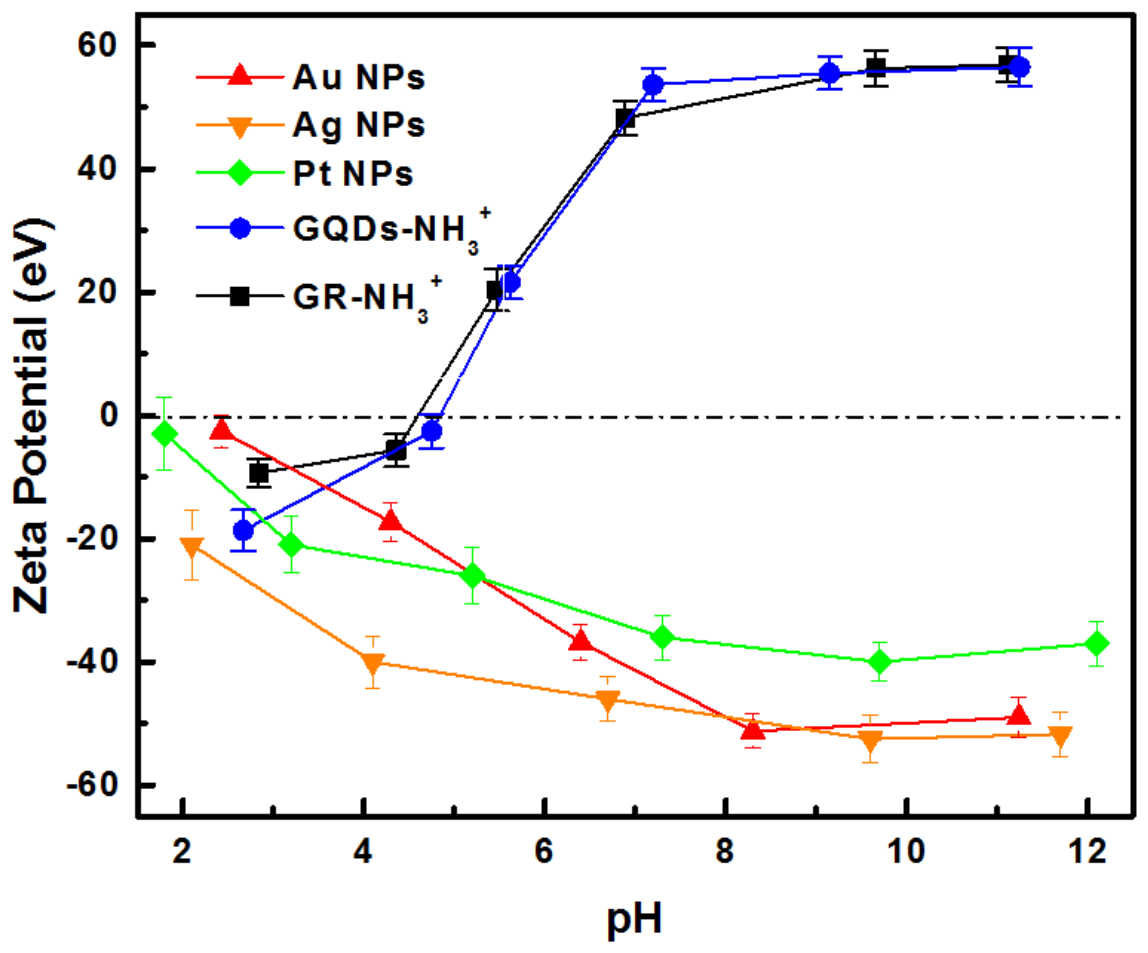

Figure 3-4. Zeta potential tests of Au, Ag, Pt NCs and GQDs-NH ${ }_{3}^{+}, \mathrm{GR}_{-} \mathrm{NH}_{3}{ }^{+}$as a function of $\mathrm{pH}$ value. 

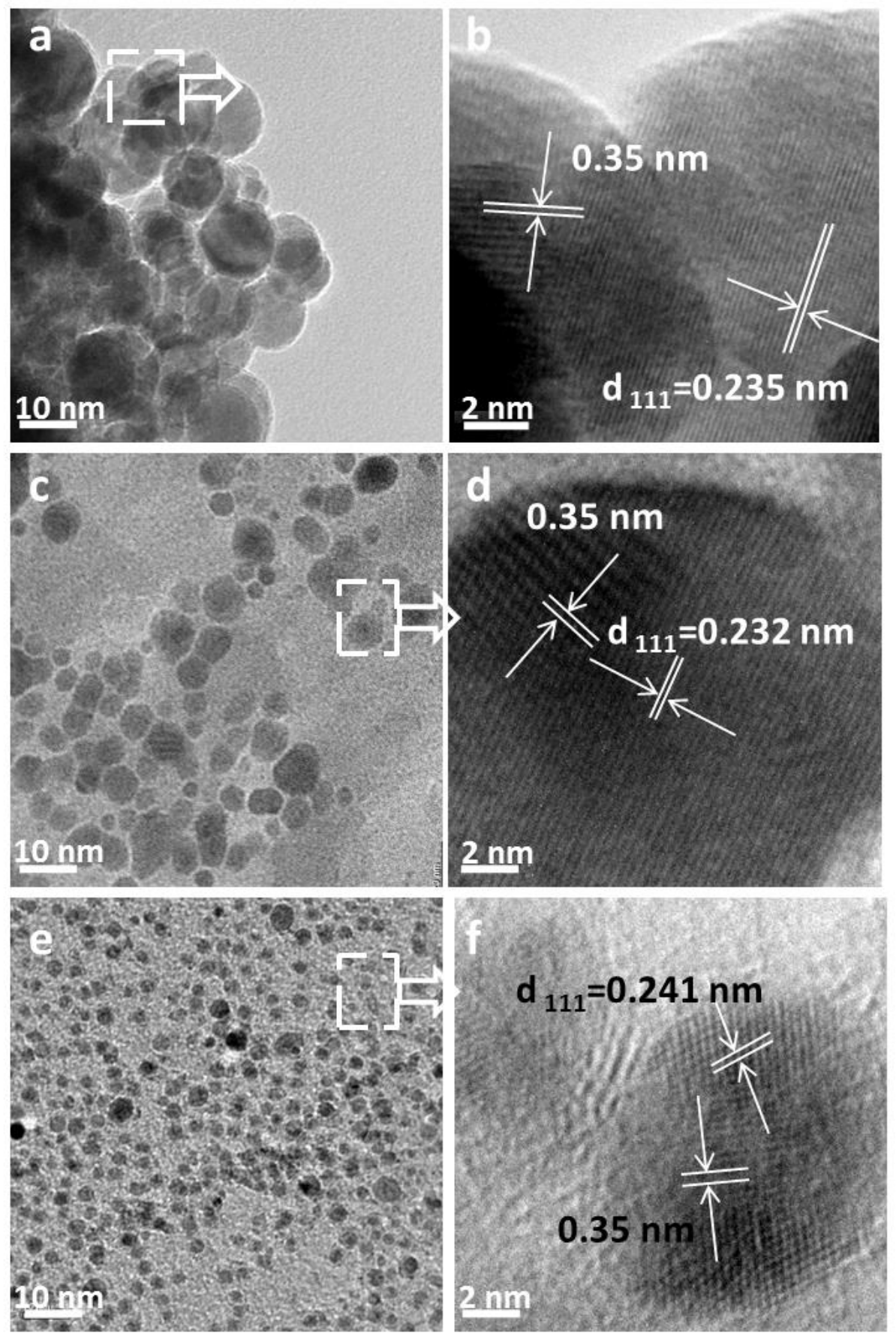

Figure 3-5. TEM and corresponding HRTEM images of (a \& b) (Au/GQDs) ${ }_{10}$, (c \& d) $(\mathrm{Ag} / \mathrm{GQDs})_{10},(\mathrm{e} \& \mathrm{f})(\mathrm{Pt} / \mathrm{GQDs})_{10}$ multilayer composite thin films.

Transmission electron microscopy (TEM) was used to probe the microscopic structure of (M/GQDs) $)_{10}$ multilayer composite films. As shown in Figure 3-5(a, c, e), $\mathrm{Au}, \mathrm{Ag}$, and $\mathrm{Pt} \mathrm{NCs}$ are uniformly distributed in the (M/GQDs) $)_{10}$ multilayer 
composite films with intimate interfacial integration with GQDs which can be unambiguously revealed by corresponding HRTEM images. As manifested in Fig 3$\mathbf{5}(\mathbf{b}, \mathbf{d}, \mathbf{f})$, the lattice fringe of $0.35 \mathrm{~nm}$ can be indexed to the (002) crystal planed of GQDs and the lattice spacing of $0.235,0.232$, and $0.241 \mathrm{~nm}$ are attributed to the crystallographic plane of face-centered cubic (fcc) Au, Ag, and Pt NCs, respectively.

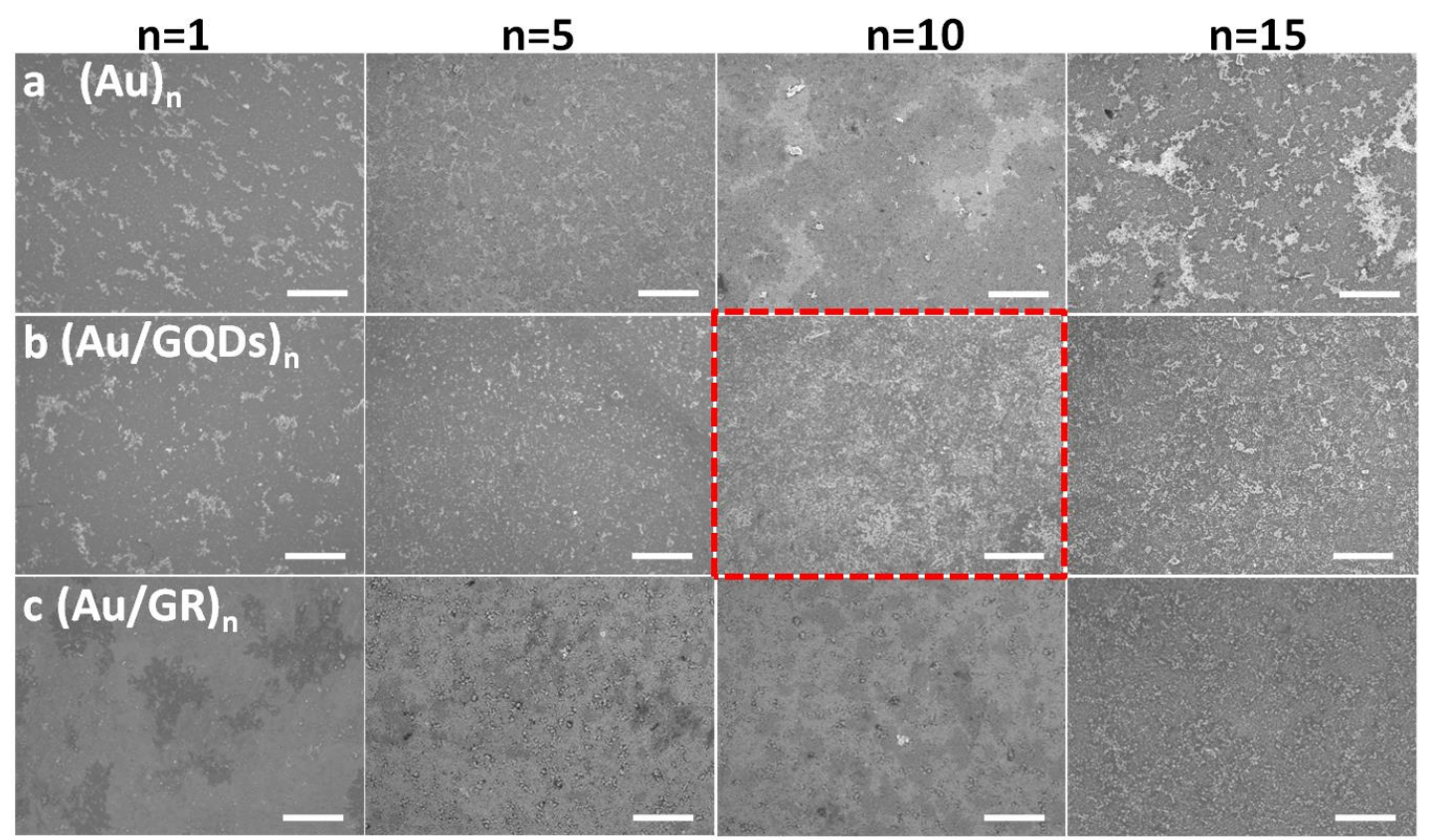

Figure 3-6. Top-view FESEM images of (a) (Au) $)_{n}$, (b) (Au/GQDs) $)_{n}$, and (c) $(\mathrm{Au} / \mathrm{GR})_{\mathrm{n}}(\mathrm{n}=\mathbf{1 , 5 , 1 0 , 1 5 )}$ multilayer thin films, scale bar is $2 \mu \mathrm{m}$.
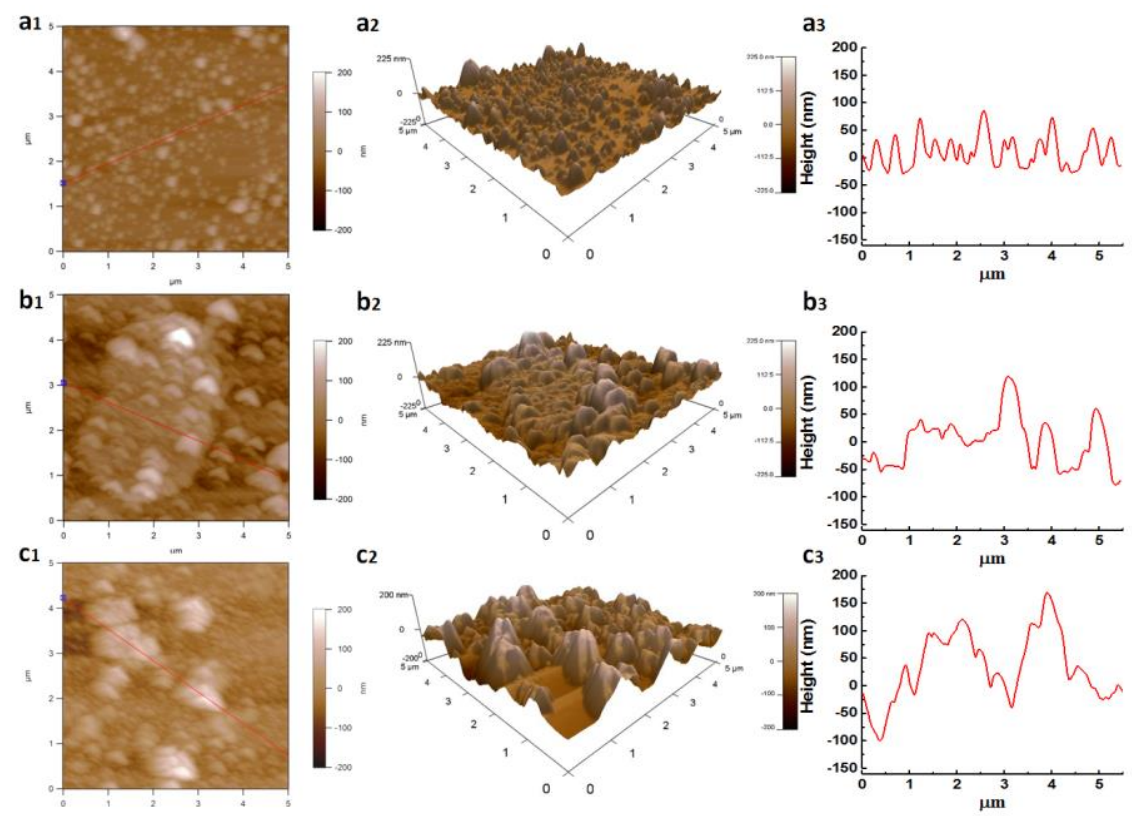
Figure 3-7. AFM images of (a) (Au/GQDs) $)_{10}$, (b) (Au/GR) $)_{10}$, and (c) $(\mathrm{Au})_{10}$ multilayer film with corresponding 3D image, and height profile of the red line that goes through the film.

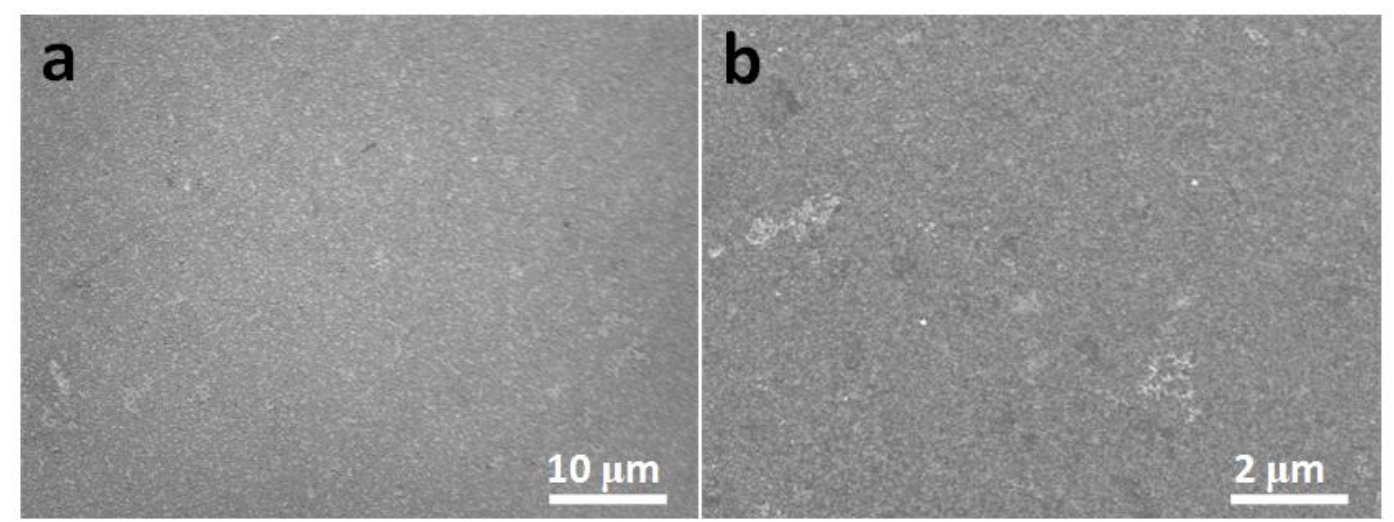

Figure 3-8. FESEM images of (a) (Ag/GQDs) $)_{10}$, and (b) (Pt/GQDs) $)_{10}$ multilayer thin film.

FESEM (Figure 3-6) and AFM images (Figure 3-7) were also utilized to probe the specific morphologies of different multilayer composite films. (Au/GQDs) $(\mathrm{n}=1,5,10,15)$ multilayer composite films exhibited relatively uniform surface with $\mathrm{Au}$ NCs evenly deposited on it and the density of Au NCs increases with increasing the assembly cycles, implying the developed LbL assembly approach based on electrostatic attractive interaction is efficient in fabricating $(\mathrm{Au} / \mathrm{GQDs})_{\mathrm{n}}$ multilayer films. This can be further corroborated by the successful fabrication of (Ag/GQDs) and $(\mathrm{Pt} / \mathrm{GQDs})_{\mathrm{n}}$ multilayer composite films, as reflected by Figure 3-8. For comparison, pure $(\mathrm{Au})_{\mathrm{n}}$ and $(\mathrm{Au} / \mathrm{GR})_{\mathrm{n}}$ counterpart multilayer films were also prepared via analogous LbL assembly rout. It is evident that $(\mathrm{Au} / \mathrm{GR})_{10}$ multilayer film demonstrated a uniform surface without obvious agglomeration of Au NCs (Figure 3-6c \& 3-7b), which is analogous to the morphology of (Au/GQDs) $)_{10}$ film. On the contrary, serious agglomeration of Au NCs was observed in the blank $(\mathrm{Au})_{\mathrm{n}}(\mathrm{n}=1,5$, 
10, 15) multilayer thin films (Figure 3-6a \& 3-7c), predominantly due to the lack of a suitable supporting substrate to stabilize the Au NCs.
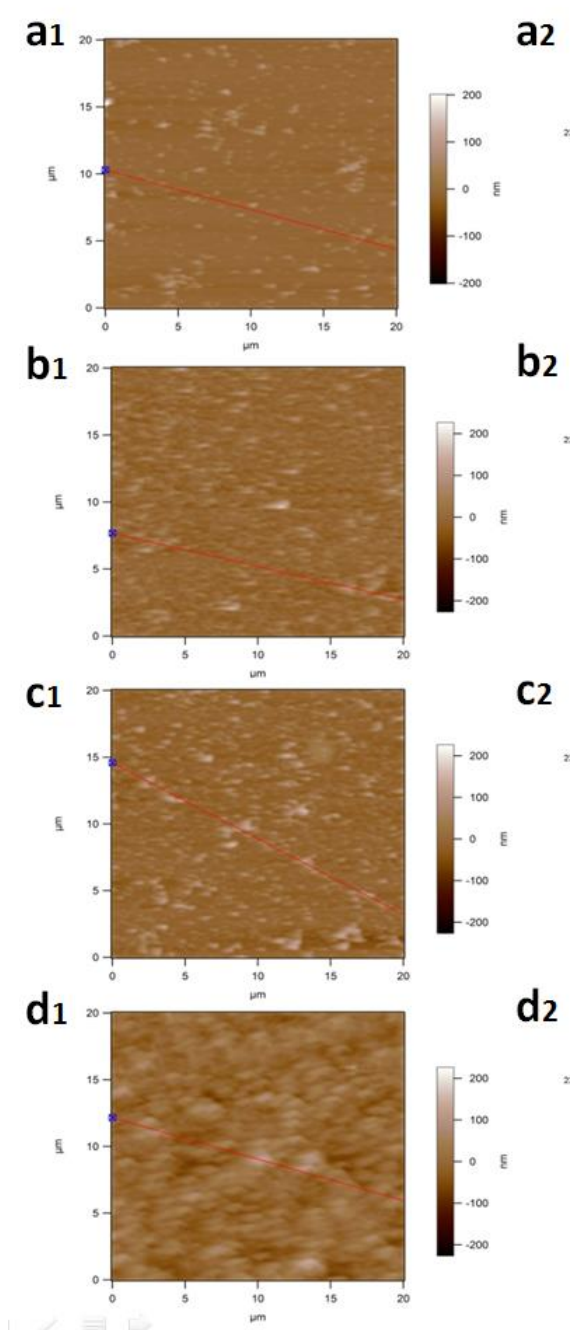

a2

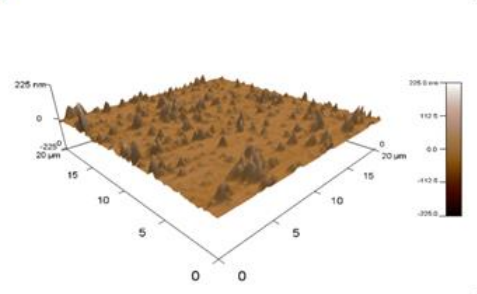

b2

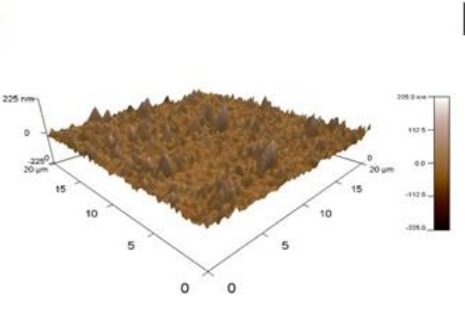

C2

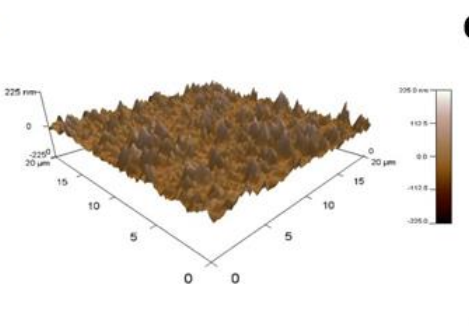

d2

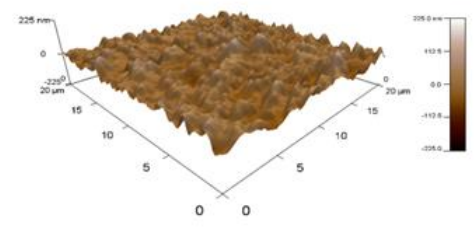

а3

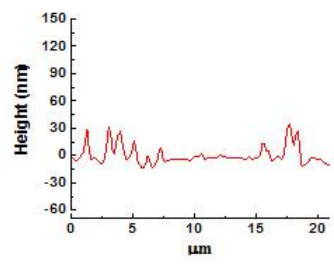

b3

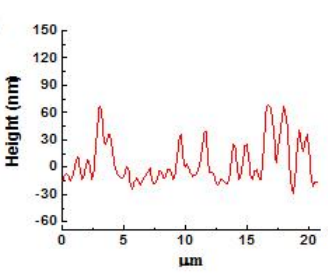

C3

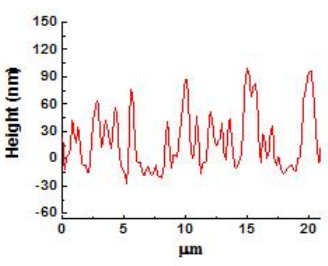

d3

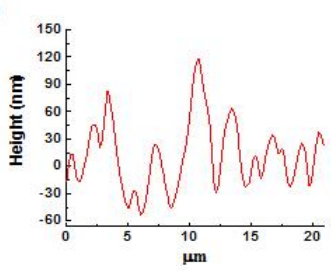

Figure 3-9. AFM image of (Au/GQDs) $n$ multilayer films with varied assembly layer (a) $n=1$, (b) $n=5$, (c) $n=10$, (d) $n=15$, along with corresponding 3D images, and height profiles.

Consistently, as shown in Figure 3-9, AFM results of $(\mathrm{Au} / \mathrm{GQDs})_{\mathrm{n}}(\mathrm{n}=1,5,10$, 15) multilayer films indicated that the thickness (Figure 3-9(a3, b3, c3)) and roughness (Figure 3-9(a2, b2, c2)) of the (Au/GQDs) films increase gradually with boosting the assembly cycle. Noteworthily, relatively smooth and flat surface was retained in the $(\mathrm{Au} / \mathrm{GQDs})_{\mathrm{n}}$ multilayer films and this is in faithful agreement with FESEM results. Furthermore, to examine the (Au/GQDs) $)_{10}$ size-dependent electron 
transport performance, we employed c-AFM to measure the nanoscale electronic property of the hybrid composite. A conductive tip sans over the sample surface to achieve charge carriers at a specific applied bias in the current imaging mode, and the nanoscale current distribution of the samples is presented in the dark current condition. The dark current images of $(\mathrm{Au})_{10}$ and $(\mathrm{Au} / \mathrm{GQDs})_{10}$ heterostructures were mapped out in Figure 3-10. The average current density (171.3 pA) of (Au/GQDs) ${ }_{10}$ heterostructure, indicated by the bright area (high-current) in the whole scanned area, is over 30 times than that $(5.1 \mathrm{pA})$ of $(\mathrm{Au})_{10}$. These results suggest that a more continuous electron transport network in the (Au/GQDs) $)_{10}$ sample leads to a more efficient electron transport, thus higher observed current and efficient catalytic performance.
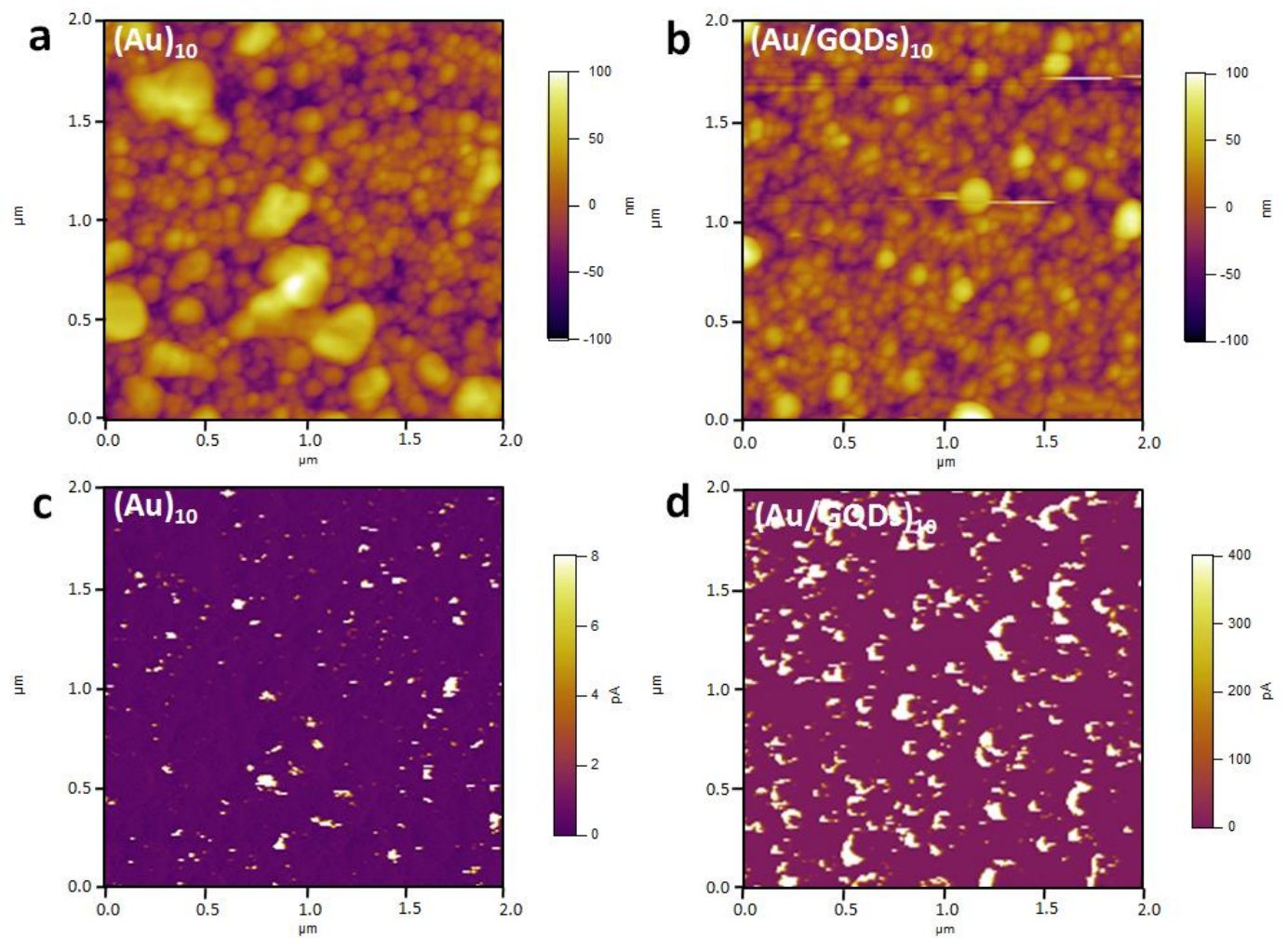

Figure 3-10. c-AFM topography (a, b) and dark current images (c, d) of the $(\mathrm{Au})_{10}$ and $(\mathrm{Au} / \mathrm{GQDs})_{10}$ electrodes with a scan size of $2 \times 2 \mu \mathrm{m}$ and a bias potential of $10 \mathrm{mV}$ applied to FTO substrate. 

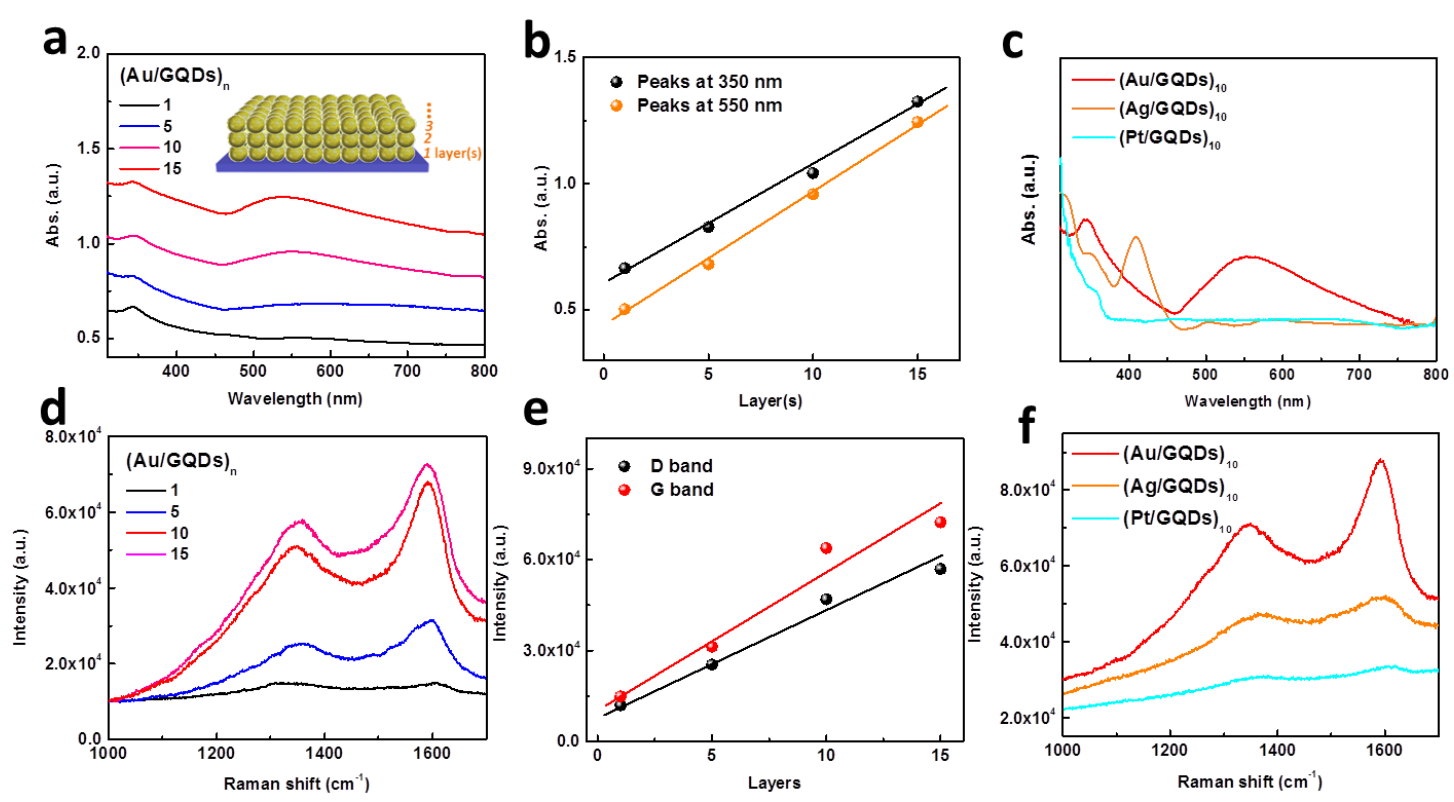

Figure 3-11. (a) UV-vis diffuse reflectance spectra (DRS) curves of (Au/GQDs) multilayer composite films, (b) the corresponding absorption peak at 350 and $550 \mathrm{~nm}$ as a function of the number of layers. (c) UV-vis DRS of (M/GQDs) 10 (M=Au, Ag, Pt) multilayer composite thin films. (d) Raman spectra of $(A u / G Q D s)_{n}$ multilayer composite films, and (e) the corresponding intensity of $D$ and $G$ bands as a function of the number of layers. (f) Raman spectra of $(\mathrm{M} / \mathrm{GQDs})_{10}(\mathrm{M}=\mathrm{Au}, \mathrm{Ag}, \mathrm{Pt})$ composite thin films.

Optical properties of $(\mathrm{M} / \mathrm{GQDs})_{\mathrm{n}}$ multilayer composite thin films were studied by UV-vis diffuse reflectance spectra (DRS). As mirrored in Figure 3-11c, DRS results of $(\mathrm{Au} / \mathrm{GQDs})_{\mathrm{n}}$ and $(\mathrm{Ag} / \mathrm{GQDs})_{10}$ multilayer films demonstrated a typical absorption peak at $350 \mathrm{~nm}$, and surface plasmon resonance (SPR) peak at 520/410 nm, which are attributed to GQDs and $\mathrm{Au} / \mathrm{Ag} \mathrm{NCs}$, respectively. Noteworthily, no obvious absorption peak was observed in the DRS result (Pt/GQDs) ${ }_{10}$ multilayer film due to the weak absorption of $\mathrm{Pt} \mathrm{NCs}$ in the visible region. Moreover, note that absorption intensity of these featured peaks increases proportionally with the assembly layer (Figure 3-11(a \& b)), strongly substantiating the gradual growth of $(\mathrm{Au} / \mathrm{GQDs})_{\mathrm{n}}$ multilayer films in which $\mathrm{Au}$ NCs and GQDs components were alternately integrated in a controllable mode via LbL assembly approach. Correspondingly, as shown in Figure 3-11(d \& e), Raman spectra of (Au/GQDs) 
$(\mathrm{n}=1,5,10,15)$ multilayer films showed that peak intensity of the typical D and $G$ bands arising from GQDs located at 1357 and $1593 \mathrm{~cm}^{-1}$ also increases proportionally with the assembly layer, thus once again verifying alternate intercalation of GQDs layers in the composite films. The typical D and G peaks of GQDs can be found in Raman results of (Ag/GQDs) ${ }_{10}$ and (Pt/GQDs) $)_{10}$ multilayer films (Figure 3-11f).
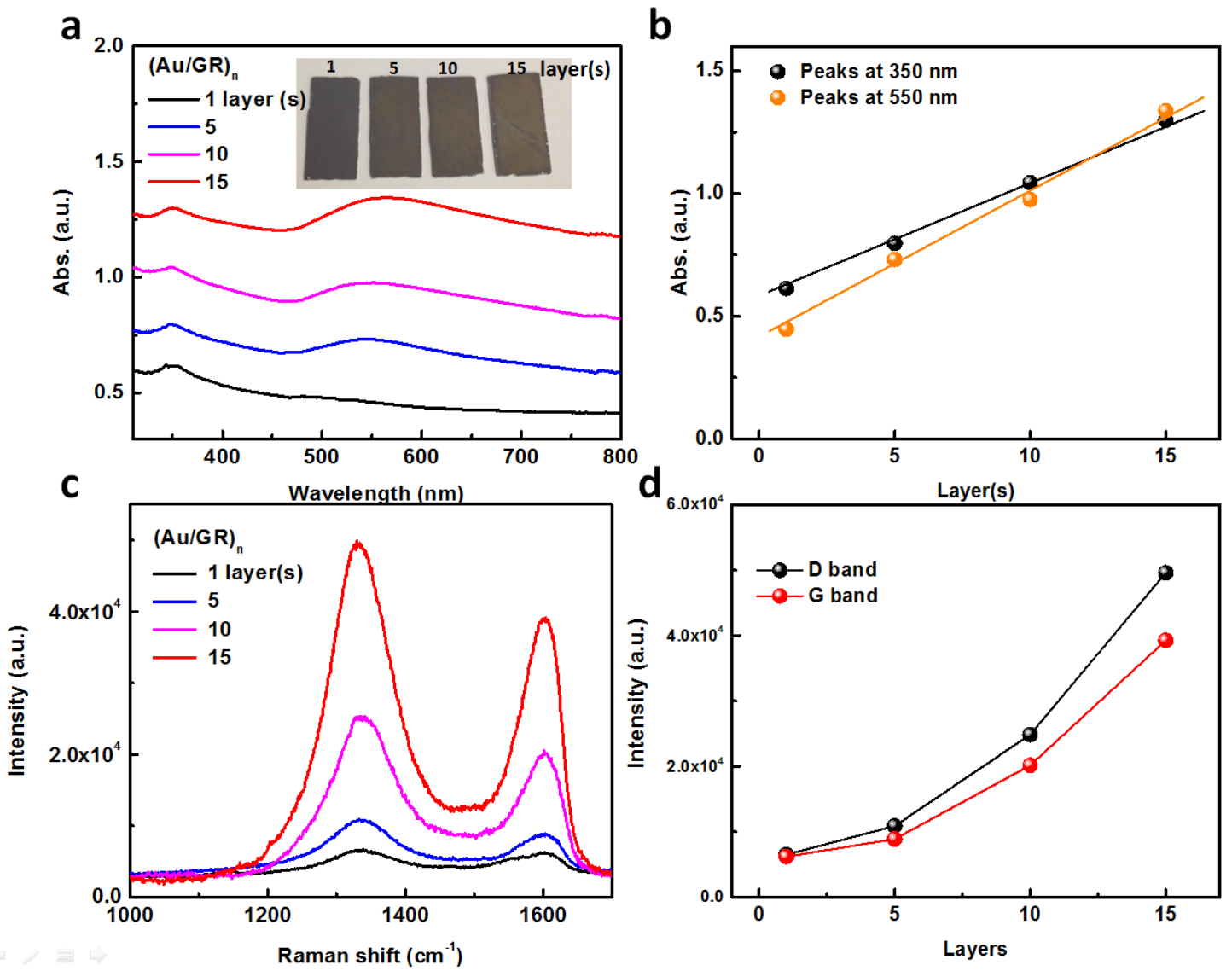

Figure 3-12. (a) UV-vis diffuse reflectance spectra (DRS) curves of (Au/GR) $(n=1,5,10,15)$ multilayer film and, (b) the corresponding absorption peak at 350 and $550 \mathrm{~nm}$ as a function of the number of layers. (c) Raman spectra of $(\mathrm{Au} / \mathrm{GR})_{\mathrm{n}}(\mathrm{n}=1,5,10,15)$ multilayer films, and (d) the corresponding intensity of $D$ and $G$ bands as a function of the number of layers.

The analogous DRS and Raman results were also observed in the $(\mathrm{Au} / \mathrm{GR})_{\mathrm{n}}$ counterpart multilayer films which were constructed by the same LbL assembly approach except for replacing 0D GQDs with 2D GR (Figure 3-12). Thus, the results concurrently evidenced the formation of multilayered nanostructures for (M/GQDs) 
$(\mathrm{M}=\mathrm{Au}, \mathrm{Ag}, \mathrm{Pt})$ and $(\mathrm{Au} / \mathrm{GR})_{\mathrm{n}}$ composite films. Furthermore, it should be noted that, distinct from Raman result of $(\mathrm{Au} / \mathrm{GR})_{\mathrm{n}}$ counterpart films (Figure 3-12c), substantial peak broadening and bathochromic shift of D and G bands were observed in the $(\mathrm{Au} / \mathrm{GQDs})_{\mathrm{n}}$ films when the assembly layer increases, indicative of more significantly enhanced interaction between $\mathrm{Au}$ NCs and GQDs in the intimately stacked 0D/0D $(\mathrm{Au} / \mathrm{GQDs})_{\mathrm{n}}$ films in comparison with that between $\mathrm{Au}$ NCs and GRs in 0D/2D $(\mathrm{Au} / \mathrm{GR})_{\mathrm{n}}$ films. $(190,191)$
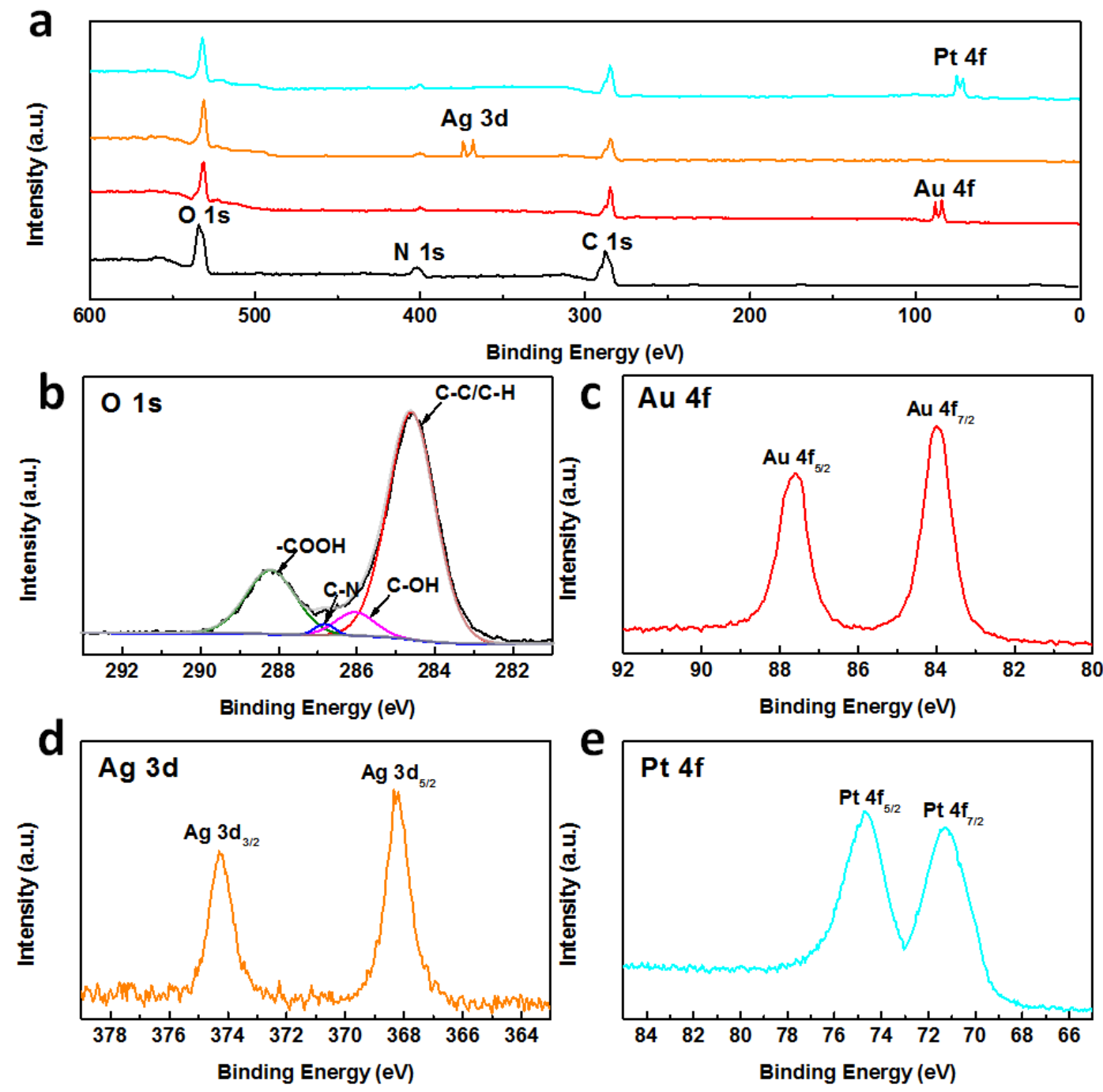

Figure 3-13. (a) XPS survey spectra of GQDs- $\mathrm{NH}_{3}{ }^{+}$and $(\mathrm{M} / \mathrm{GQDs})_{\mathrm{n}}(\mathrm{M}=\mathrm{Au}, \mathrm{Ag}$, $\mathrm{Pt}$ ) multilayer composite thin films and high-resolution spectra of (b) $\mathrm{C} \mathrm{1s}$, and (c) Au $4 \mathrm{f}$ for (Au/GQDs) ${ }_{10}$, and (d) Ag 3d for (Ag/GQDs) $)_{10}$, and (e) Pt 4 for (Pt/GQDs) $)_{10}$. 
$\mathrm{X}$-ray photoelectron spectroscopy (XPS) is used to explore the composition and elemental chemical states of (M/GQDs) $)_{n}$ multilayer films. Figure 3-13a shows the survey spectra of $(\mathrm{M} / \mathrm{GQDs})_{10}(\mathrm{M}=\mathrm{Au}, \mathrm{Ag}, \mathrm{Pt})$ multilayer composite films and GQDs- $\mathrm{NH}_{3}{ }^{+}$, for which the characteristic signals of $\mathrm{C} 1 \mathrm{~s}, \mathrm{~N} 1 \mathrm{~s}, \mathrm{O}$ 1s originate from GQDs- $\mathrm{NH}_{3}{ }^{+}$, and $\mathrm{Au} 4 \mathrm{f}, \mathrm{Ag} \mathrm{3d}$, as well as $\mathrm{Pt} 4 \mathrm{f}$ signals arise from metal NCs, indicative of successful combination of metal NCs and GQDs- $\mathrm{NH}_{3}{ }^{+}$in the multilayer composite films. Alternatively, with a view to differentiating the elemental state and specific chemical bond species of the films, high-resolution C 1s spectra of $(\mathrm{M} / \mathrm{GQDs})_{10}$ multilayer thin films (Figure 3-13b and Figure 3-14) can be deconvoluted to four peaks with binding energy of $284.60,286.15,286.85$, and $288.20 \mathrm{eV}$, corresponding to $\mathrm{C}-\mathrm{C} / \mathrm{C}-\mathrm{H}, \mathrm{C}-\mathrm{OH}, \mathrm{C}-\mathrm{N}$, and $\mathrm{COOH}$ species from GQDs$\mathrm{NH}_{3}{ }^{+}$, respectively. The featured $\mathrm{Au} 4 \mathrm{f}_{7 / 2}$ and $\mathrm{Au} 4 \mathrm{f}_{5 / 2}$ peaks with binding energy of 84.05, and $87.75 \mathrm{eV}$ (Figure 3-13c) are correspond to metallic $\mathrm{Au}^{0} .(192)$ Analogously, as shown in Figure 3-13d, high-resolution $\mathrm{Ag} 3 \mathrm{~d}$ spectrum with two Ag $3 d_{5 / 2}$ and $\mathrm{Ag} 3 \mathrm{~d}_{3 / 2}$ peaks positioned at 368.20 and $374.25 \mathrm{eV}$ corroborates the metallic $\mathrm{Ag}^{0}$.(192) High-resolution spectrum of Pt 4f with two peaks located at 71.25 $\left(\mathrm{Pt} 4 \mathrm{f}_{7 / 2}\right)$ and $74.70 \mathrm{eV}\left(\mathrm{Pt} 4 \mathrm{f}_{5 / 2}\right)$ (Figure 3-13e) assure the metallic $\mathrm{Pt}^{0} .(192)$ Therefore, XPS spectra of $\mathrm{Au} 4 \mathrm{f}, \mathrm{Ag} \mathrm{3d}$, and Pt 4f prove that elemental chemical states of metal NCs were retained in the composite films when they were combined with GQDs via pronounced electrostatic interaction through LbL self-assembly approach. Alternatively, XRD patterns (Figure 3-15) of (M/GQDs) $)_{10}$ multilayer composite films also verified the integration of the metal NCs in the multilayer composite films. 

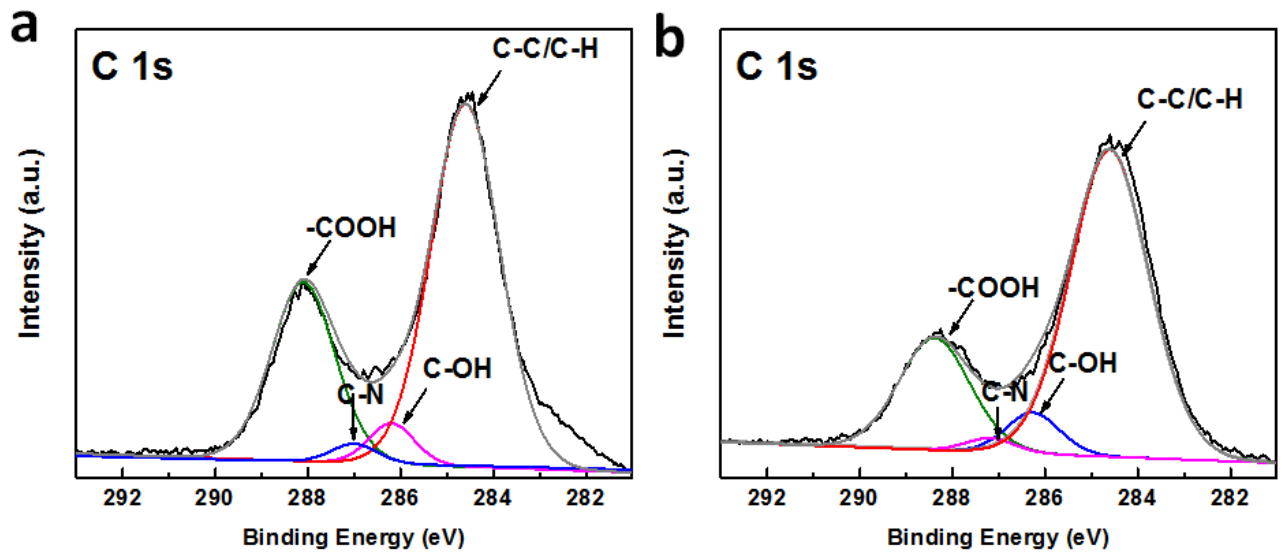

Figure 3-14. High-resolution spectra of C 1s for (Ag/GQDs) ${ }_{10}$ and (Pt/GQDs) $)_{10}$.

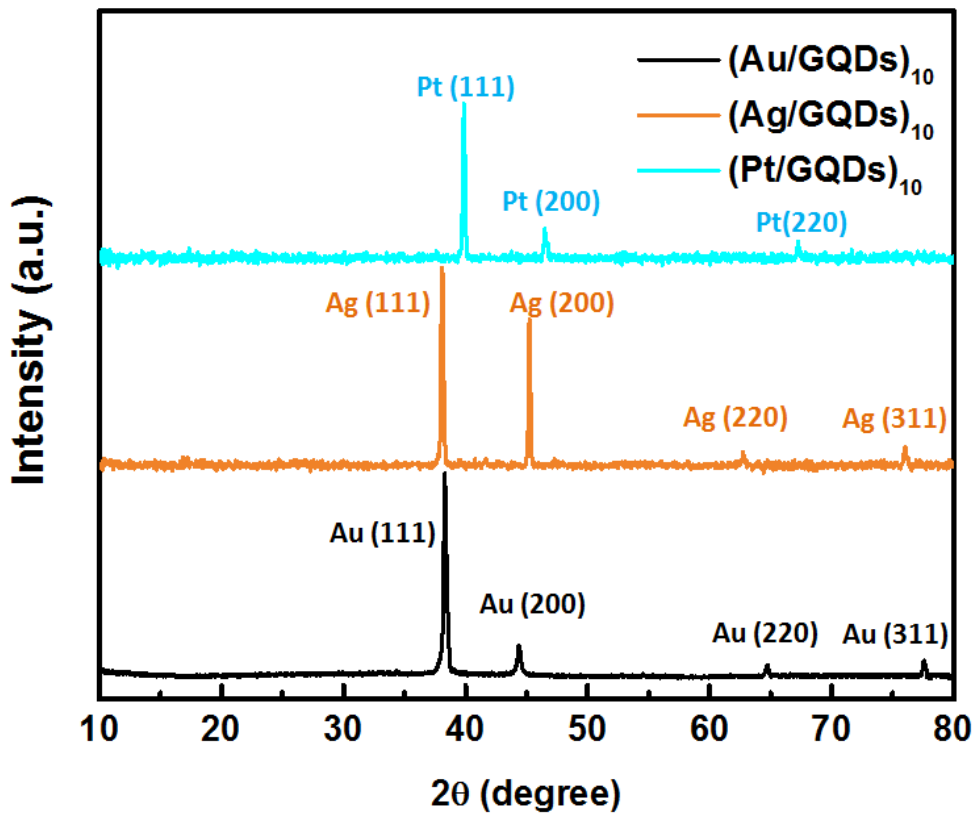

Figure 3-15. XRD patterns of (Au/GQDs) $)_{10}$, (Ag/GQDs) $)_{10}$ and (Pt/GQDs) multilayer composite film. 
3.3.2 Selective catalytic reaction performance of $(\mathrm{M} / \mathrm{GQDs})_{\mathrm{n}}(\mathrm{M}=\mathrm{Au}, \mathrm{Ag}, \mathrm{Pt})$ hybrid nanostructures
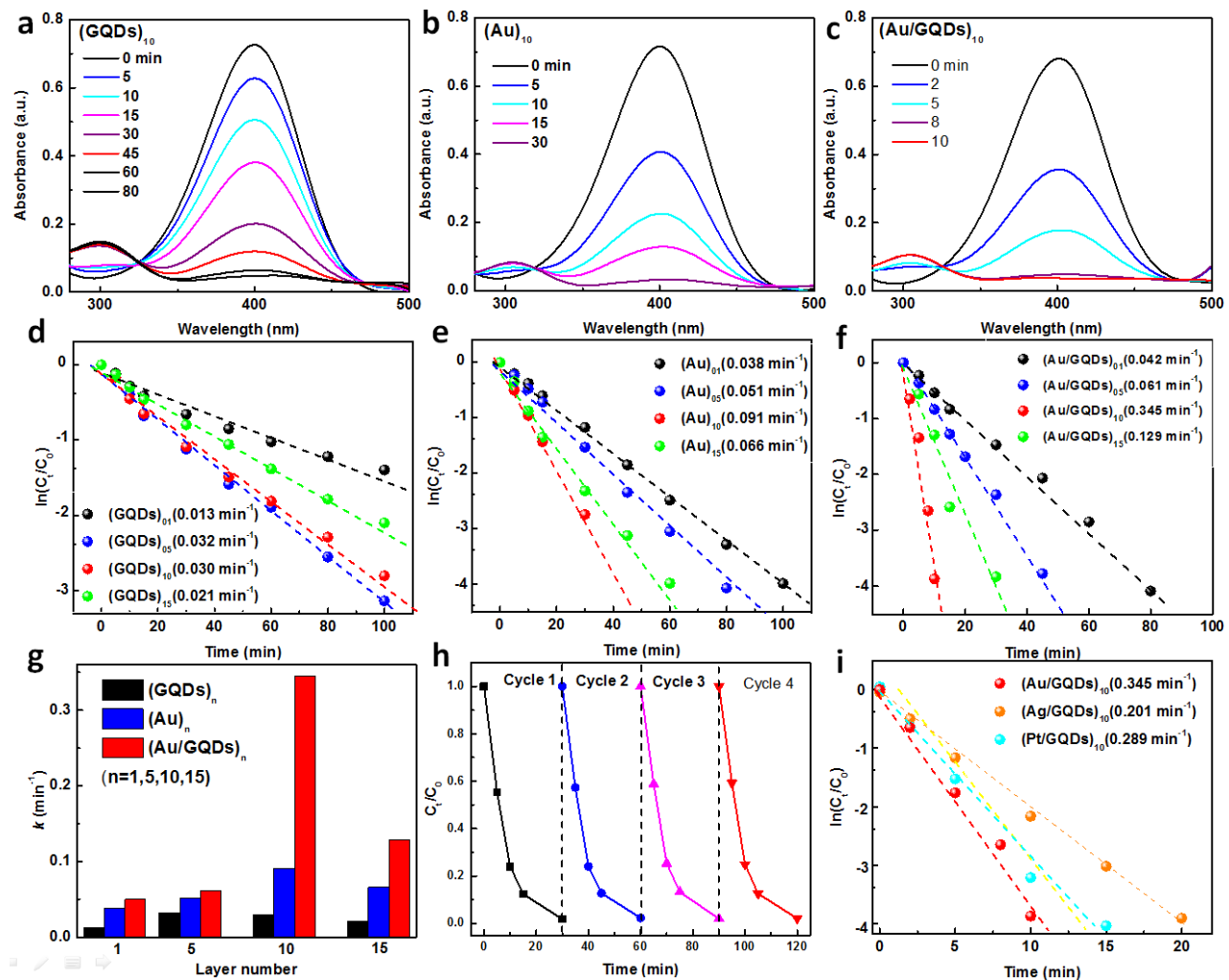

Figure 3-16. (a-c) Time-dependent UV-vis absorption spectra for catalytic reduction of 4-NP to 4-AP over (a) (GQDs) $)_{10}$, (b) (Au) $)_{10}$, and (c) (Au/GQDs) multilayer films. Plots of $\ln \left(C_{t} / C_{0}\right)$ versus reaction time for reduction of 4-NP over (d) (GQDs) $)_{10}$, (e) (Au) $)_{10}$, and (f) (Au/GQDs) 10 multilayer films by monitoring the decreased peak intensity of $4-\mathrm{NP}$ at $400 \mathrm{~nm}$. (g) Comparison of the reaction rate constants of different multilayer films. (h) Cycling catalytic reduction reactions over (Au/GQDs) 10 multilayer films. (i) Comparison results of catalytic reduction performances over $(\mathrm{M} / \mathrm{GQDs})_{10}(\mathrm{M}=\mathrm{Au}, \mathrm{Ag}, \mathrm{Pt})$ multilayer films with the same assembly layer.

Chemical reduction of 4-nitrophenol (4-NP) to 4-aminophenol (4-AP) in aqueous media under ambient conditions was used as probe reaction to evaluate the catalytic performances of different multilayer composite films. As shown in UV-vis absorption spectra of 4-NP in Figure 3-16(a-c), a featured peak at $400 \mathrm{~nm}$ corresponding to 4-NP gradually attenuated while the peak at $300 \mathrm{~nm}$ attributable to 4-AP increased concomitantly, which suggested successful reduction of 4-NP to 4-AP. 
It has been well-established that catalytic selective reduction of 4-NP to 4-AP follows the pseudo-first-order reaction kinetic.(192) As displayed in Figure 3-16(d, e, f), catalytic performances of all multilayer films are closely related to assembly layer, among which $(\mathrm{Au} / \mathrm{GQDs})_{10}$ film demonstrated the highest rate constant $\left(0.345 \mathrm{~min}^{-1}\right)$ in comparison with $(\mathrm{Au})_{10}\left(0.091 \mathrm{~min}^{-1}\right)$ and $(\mathrm{GQDs})_{10}\left(0.030 \mathrm{~min}^{-1}\right)$ counterpart films with the same assembly layer, suggesting that synergistic effect (Figure 3-16g) between $\mathrm{Au}$ NCs and GQDs contributes to the remarkably enhanced catalytic performances of (Au/GQDs $)_{10}$ films.
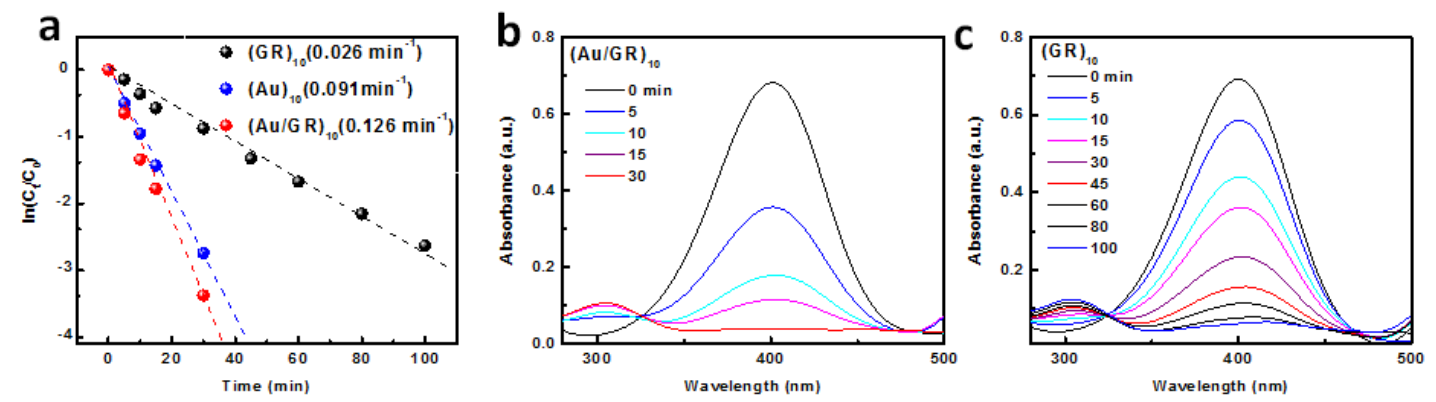

Figure 3-17. (a) Plots of $\ln \left(C_{t} / C_{0}\right)$ versus reaction time for reduction of 4-NP over different films. Time-dependent $\mathrm{UV}$-vis absorption spectra for reduction of 4-NP over (b) (GR) 10 and (c) (Au/GR) $)_{10}$ multilayer thin films catalysts.
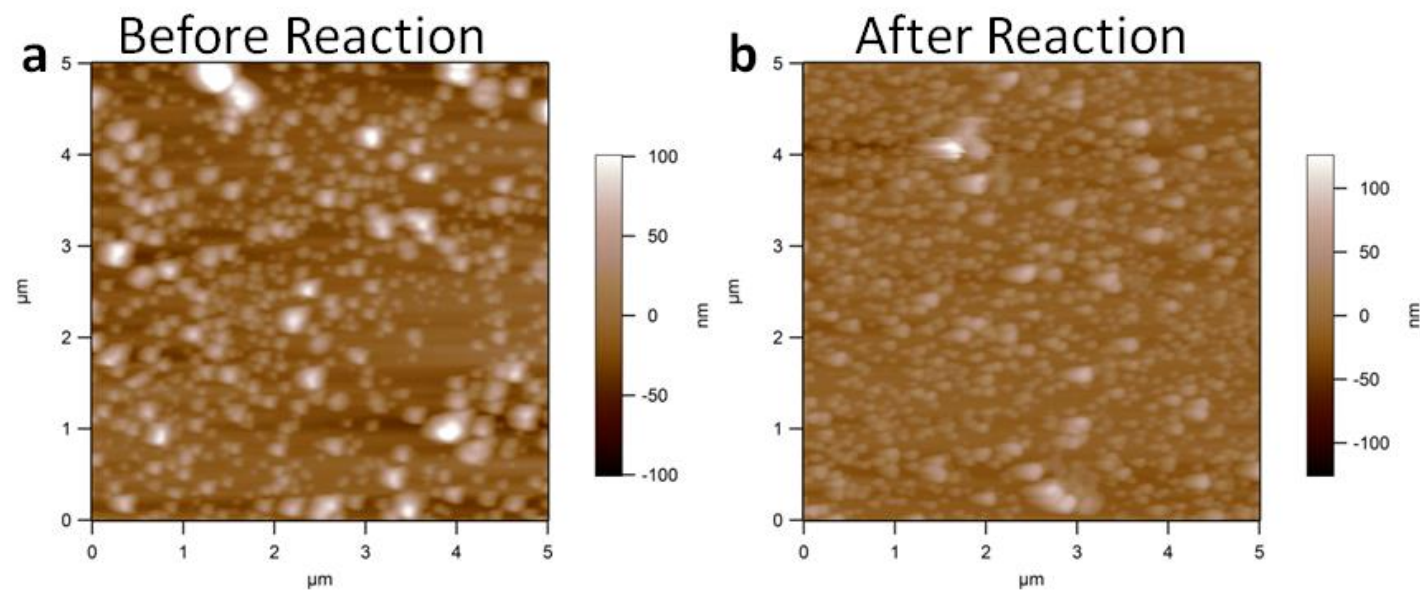

Figure 3-18. AFM images of (Au/GQDs) 10 multilayer film before (a) and after (b) catalytic reduction reactions.

Analogous results were observed in the catalytic performance of $(\mathrm{Au} / \mathrm{GR})_{10}$ multilayer films (Figure 3-17) which also exhibited much higher catalytic activities 
than $(\mathrm{Au})_{10}$ and $\mathrm{GR}_{10}$ films under the same conditions. The results indicated that synergistic interaction between $\mathrm{Au} \mathrm{NCs}$ and $\mathrm{GR}$ in $(\mathrm{Au} / \mathrm{GR})_{10}$ films are indeed responsible for the enhanced catalytic activities and this could be attributed to the intimate interfacial interaction between metal $(\mathrm{Au})$ and carbon materials (GQDs and GR) afforded by LbL assembly. It is worth noting that (Au/GQDs) ${ }_{10}$ films exhibited substantially improved catalytic activities relative to $(\mathrm{Au} / \mathrm{GR})_{10}$ films. The rate constant of $(\mathrm{Au} / \mathrm{GQDs})_{10}$ film is almost three times higher than that of $(\mathrm{Au} / \mathrm{GR})_{10}$ under the same conditions, i.e., (0.345 vs. $\left.0.126 \mathrm{~min}^{-1}\right)$, implying GQDs is much more active than GR in boosting the selective catalytic reduction performances of multilayer composite films. This observation can be attributed to the different dimensionality between GQDs and GR, which affords varied number of active sites. Apparently, 0D GQDs possesses a great number of active sites on the planar surface especially on the edge in terms of its ultra-small size, thereby facilitating more efficient adsorption and reaction of reactants compared with 2D GR. Besides, $(\mathrm{Au} / \mathrm{GQDs})_{10}$ film demonstrated favorable catalytic stability which can be reflected by the cycling experiments. Specifically, as shown in Figure 3-16h, no attenuation of catalytic performance was observed over (Au/GQDs) $)_{10}$ film even after four successive recycles, thus confirming $(\mathrm{Au} / \mathrm{GQDs})_{10}$ film is stable during the catalytic reaction. Consistently, as revealed by AFM images (Figure 3-18), uniform structure of $(\mathrm{Au} / \mathrm{GQDs})_{10}$ multilayer film was retained after cycling catalytic reactions. The promising catalytic reduction performances of other $(\mathrm{M} / \mathrm{GQDs})_{10}(\mathrm{M}=\mathrm{Ag}, \mathrm{Pt})$ multilayer films (Figure 3-16i) were also probed, among which (Au/GQDs) ${ }_{10}$ demonstrated the most efficient catalytic activity. The predominant reason accounting for the most remarkably enhanced catalytic activities of (Au/GQDs) $)_{n}$ multilayer films might be ascribed to the dual functionality of GQDs layer which facilitates the 
adsorption of 4-NP molecular owing to analogous structure, thus boosting the reaction kinetics, preventing the agglomeration of metal NCs, and enhancing the stability of multilayer composite film. It is because the average diameters of $\mathrm{Ag}$ and Pt NPs are smaller than that of Au NPs (Figure 3-2), thus providing smaller space in the multilayer nanobuilding blocks and inhibiting the diffusion and transfer of aromatic compounds during the selective catalytic reduction reaction. Therefore, $(\mathrm{Au} / \mathrm{GQDs})_{10}$ demonstrated higher efficient catalytic activity in organic transformation of aromatic nitro compounds.

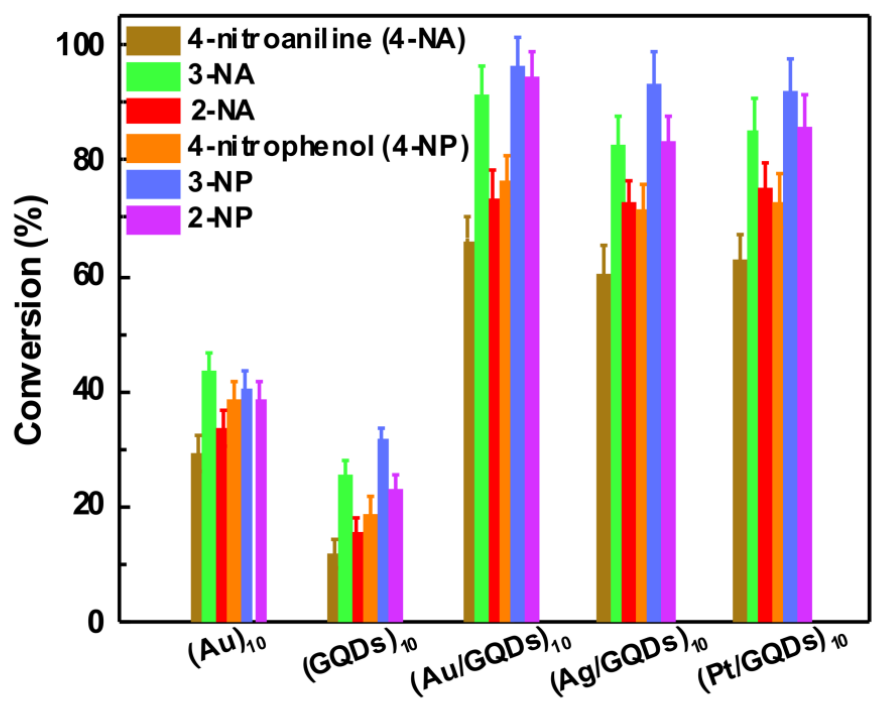

Figure 3-19. Selective catalytic reduction of aromatic nitro compounds including 4-NA, 3-NA, 2-NA, 4-NP, 3-NP, and 2-NP over (Au) ${ }_{10},(G Q D s)_{10},(A u / G Q D s)_{10}$, $(\mathrm{Ag} / \mathrm{GQDs})_{10}$, and (Pt/GQDs) $)_{10}$ multilayer thin films for the same reaction time (10 min) at ambient conditions.

To demonstrate the versatile catalytic properties of these LbL assembled multilayer films, selective catalytic performances of these films toward reduction of other aromatic nitro compounds including 2-NP, 3-NP, 4-NP, 2-NA, and 3-NA to corresponding amino compounds were further explored. As shown in Figure 3-19, consistently, $(\mathrm{M} / \mathrm{GQDs})_{10}(\mathrm{M}=\mathrm{Au}, \mathrm{Ag}, \mathrm{Pt})$ multilayer films demonstrated markedly 
enhanced catalytic activities toward the reduction of a series of aromatic nitro compounds at ambient conditions in comparison with $(\mathrm{Au})_{10}$ and (GQDs) $)_{10}$ counterparts multilayer thin films under the same conditions; catalytic performances of the different films followed the same order of: (M/GQDs $)_{10}>(\mathrm{M})_{10}>(\mathrm{GQDs})_{10}$, verifying the synergistic interaction of metal NCs and GQDs. Additionally, it was found that $(\mathrm{Au} / \mathrm{GQDs})_{10}$ film always demonstrated the most enhanced catalytic performances among $(\mathrm{M} / \mathrm{GQDs})_{10}(\mathrm{M}=\mathrm{Au}, \mathrm{Ag}, \mathrm{Pt})$ films implying metal-dependent catalytic activities of multilayer films. The corresponding reaction rate constants of $(\mathrm{Au})_{10},(\mathrm{GQDs})_{10}$, and $(\mathrm{M} / \mathrm{GQDs})_{10}(\mathrm{M}=\mathrm{Au}, \mathrm{Ag}, \mathrm{Pt})$ multilayer thin films were summarized in Table 3-1. The highly efficient catalytic activities and stability of the $(\mathrm{M} / \mathrm{GQDs})_{10}$ multilayer composite films are of great significance for wide promising practical applications, especially in aqueous media. There is no necessary separation of catalyst from the solution due to the direct growth of multilayer films on the substrate.

Table 3-1. Catalytic reaction rate constants of different multilayer thin films toward reduction of aromatic nitro compounds.

\begin{tabular}{|c|c|c|c|c|c|c|}
\hline Sample & 4-NA & 3-NA & 2-NA & 4-NP & 3-NP & 2-NP \\
\hline$(\mathrm{Au})_{10}$ & 0.091 & 0.103 & 0.097 & 0.102 & 0.118 & 0.109 \\
\hline$(\mathrm{GQDs})_{10}$ & 0.030 & 0.038 & 0.034 & 0.041 & 0.051 & 0.043 \\
\hline$(\mathrm{Au} / \mathrm{GQDs})_{10}$ & 0.345 & 0.406 & 0.354 & 0.398 & 0.417 & 0.403 \\
\hline$(\mathrm{Ag} / \mathrm{GQDs})_{10}$ & 0.201 & 0.346 & 0.312 & 0.304 & 0.386 & 0.355 \\
\hline$(\mathrm{Pt} / \mathrm{GQDs})_{10}$ & 0.289 & 0.353 & 0.337 & 0.307 & 0.369 & 0.363 \\
\hline
\end{tabular}

Unit: $\mathrm{min}^{-1}$ 
3.3.3 Electrochemical catalytic performance of $(\mathrm{M} / \mathrm{GQDs})_{\mathrm{n}}(\mathrm{M}=\mathrm{Au}, \mathrm{Ag}, \mathrm{Pt})$ hybrid nanostructures
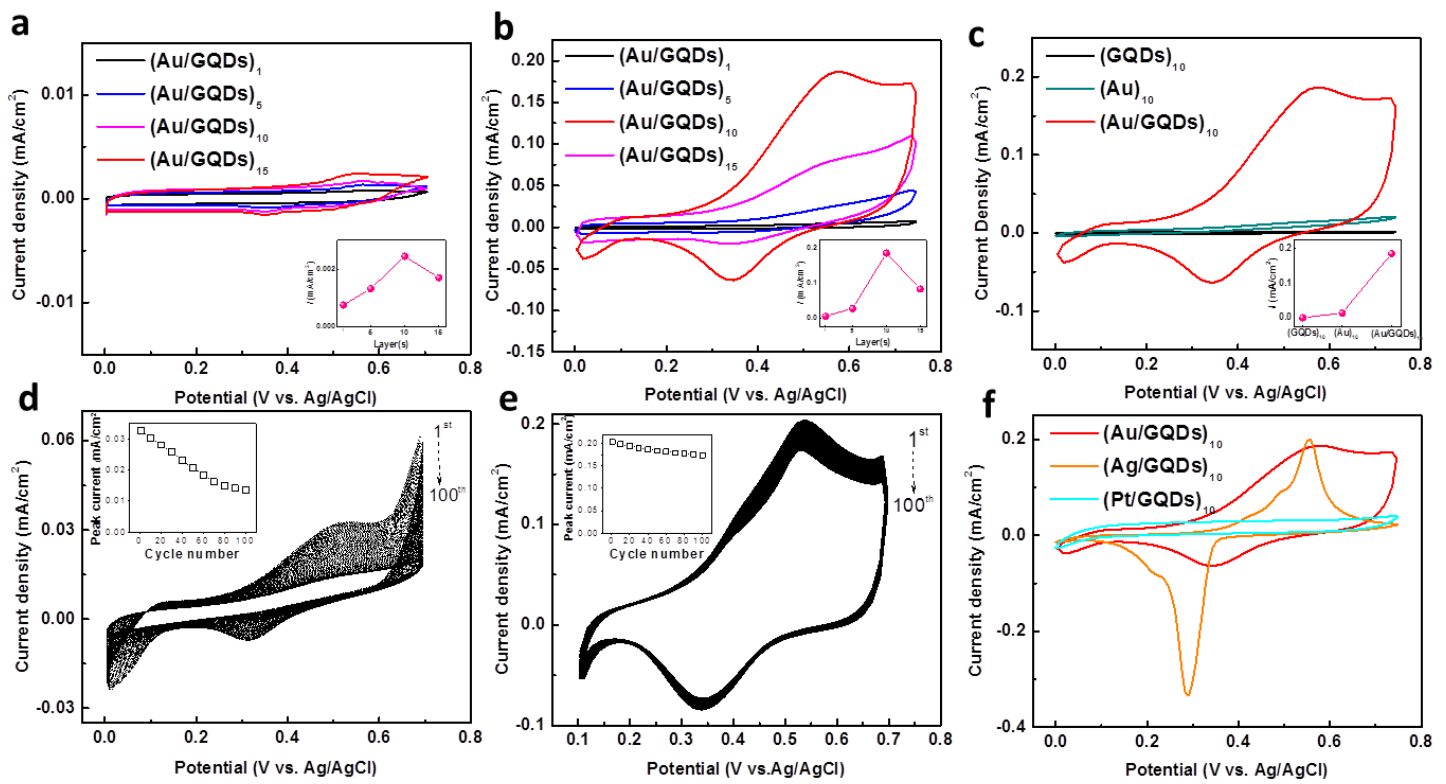

Figure 3-20. Cyclic voltammograms (CV) results of (Au/GQDs) $)_{n}$ multilayer films measured in 0.10 M KOH (a) without and (b) with 1.0 M methanol, and inset of the plots showed the anodic peak current for (Au/GQDs) multilayer films measured in the forward sweep. (c) CV results of (GQDs) $)_{10},(\mathrm{Au})_{10}$, and (Au/GQDs) $)_{10}$ multilayer films measured in $0.10 \mathrm{M} \mathrm{KOH}$ with 1.0 $\mathrm{M}$ methanol in a $\mathrm{N}_{2}$ atmosphere. Stability test of (d) (Au) $)_{10}$ and (e) (Au/GQDs) $)_{10}$ multilayer films, (f) $\mathrm{CV}$ results of $(\mathrm{M} / \mathrm{GQDs})_{10}(\mathrm{M}=\mathrm{Au}, \mathrm{Ag}, \mathrm{Pt})$ multilayer films.

Apart from selective catalytic transformation performances, electrochemical performances of $(\mathrm{Au} / \mathrm{GQDs})_{\mathrm{n}}$ multilayer films toward methanol oxidation were also probed by using cyclic voltammetry $(\mathrm{CV})$ technique in $\mathrm{KOH}$ solution $(0.1 \mathrm{M})$ without and with methanol in $\mathrm{N}_{2}$ atmosphere. As shown in Figure 3-20a, without methanol, an obvious onset potential of $0.52 \mathrm{~V}(v s . \mathrm{Ag} / \mathrm{AgCl})$ was observed in the $\mathrm{CV}$ results of $(\mathrm{Au} / \mathrm{GQDs})_{\mathrm{n}}$ multilayer films in the forward sweep, which is ascribed to the adsorption of $\mathrm{OH}^{-}$and then generation of $\mathrm{AuO}$ on the surface at a larger potential as depicted as the following reactions.

$$
\begin{aligned}
& \mathrm{Au}+\mathrm{OH}^{-} \rightarrow \mathrm{Au}-\mathrm{OH}_{\mathrm{ads}}{ }^{(1-\lambda)-}+\lambda \mathrm{e}^{-} \\
& \mathrm{Au}-\mathrm{OH}_{\mathrm{ads}}{ }^{(1-\lambda)-}+\mathrm{OH}^{-} \rightarrow \mathrm{AuO}+\mathrm{H}_{2} \mathrm{O}+(2-\lambda) \mathrm{e}^{-}
\end{aligned}
$$


where $\mathrm{OH}_{\text {ads }}$ means the adsorbed $\mathrm{OH}^{-}$on $\mathrm{Au} \mathrm{NCs}$, and $\lambda$ means the coefficient of charge transfer, which changes between 0 (non-faradaic) and 1 (faradaic).

When methanol $(1.0 \mathrm{M})$ was mixed to the reaction solution, CV results (Figure 3-20b) of $(\mathrm{Au} / \mathrm{GQDs})_{\mathrm{n}}$ is remarkably different from those obtained in the absence of methanol (Figure 3-20a); for instance, a substantial anodic peak at $0.52 \mathrm{~V}$ ( $v s$. $\mathrm{Ag} / \mathrm{AgCl})$ and a cathodic current peak at $0.35 \mathrm{~V}(v s . \mathrm{Ag} / \mathrm{AgCl})$ were clearly observed for $(\mathrm{Au} / \mathrm{GQDs})_{10}$ film. Noteworthily, $(\mathrm{Au} / \mathrm{GQDs})_{10}$ film exhibited the optimal electrocatalytic activity in comparison with other counterpart films with varied assembly layer. Generally, current density of (Au/GQDs) $)_{n}$ films increases with increasing the number of assembly layer to 10 and then, current density decreased when assembly layer is more than 10 (Figure 3-20b). The result suggests that electrocatalytic performances of (Au/GQDs $)_{n}$ films for methanol oxidation can be changed by the assembly layer. Moreover, it was revealed by Figure 3-20c, analogous to selective catalytic reduction performance, (Au/GQDs) $)_{10}$ multilayer composite film once again demonstrated the optimal electrocatalytic activity toward methanol oxidation with substantially enhanced anodic current and low potential relative to $(\mathrm{Au})_{10}$ and $(\mathrm{GQDs})_{10}$ film, thus strongly evidencing the synergistic effect of $\mathrm{Au}$ NCs and GQDs in boosting the electrocatalytic performance of (Au/GQDs) ${ }_{10}$ toward methanol oxidation. Electrocatalytic stability of (M/GQDs) multilayer films are of paramount importance for actual utilization of direct methanol fuel cell. As reflected in Fig 3-20e, (Au/GQDs) $)_{10}$ multilayer film retained significantly high current density of $0.174 \mathrm{~mA} / \mathrm{cm}^{2}$ which accounts for a retention of ca. $89 \%$ original current density after 100 cycles of CV measurements, exhibiting favorable electrocatalytic stability. On the contrary, as shown in Figure 3-20d, free $(\mathrm{Au})_{10}$ multilayer film only exhibited a remarkably lower cyclic instability of $41 \%$ and a 
smaller current density $\left(0.0113 \mathrm{~mA} / \mathrm{cm}^{2}\right)$ in comparison with $(\mathrm{Au} / \mathrm{GQDs})_{10}$ composite film. The result highlights the important role of GQDs as a stable ingredient protecting the catalytically active sites of Au NCs during electrochemical progress. More significantly, apart from the (Au/GQDs) $)_{\mathrm{n}}$ film, (Ag/GQDs) $)_{10}$ and (Pt/GQDs) ${ }_{10}$ multilayer composite film also demonstrated highly efficient electrocatalytic performances toward methanol oxidation (Figure 3-20f).
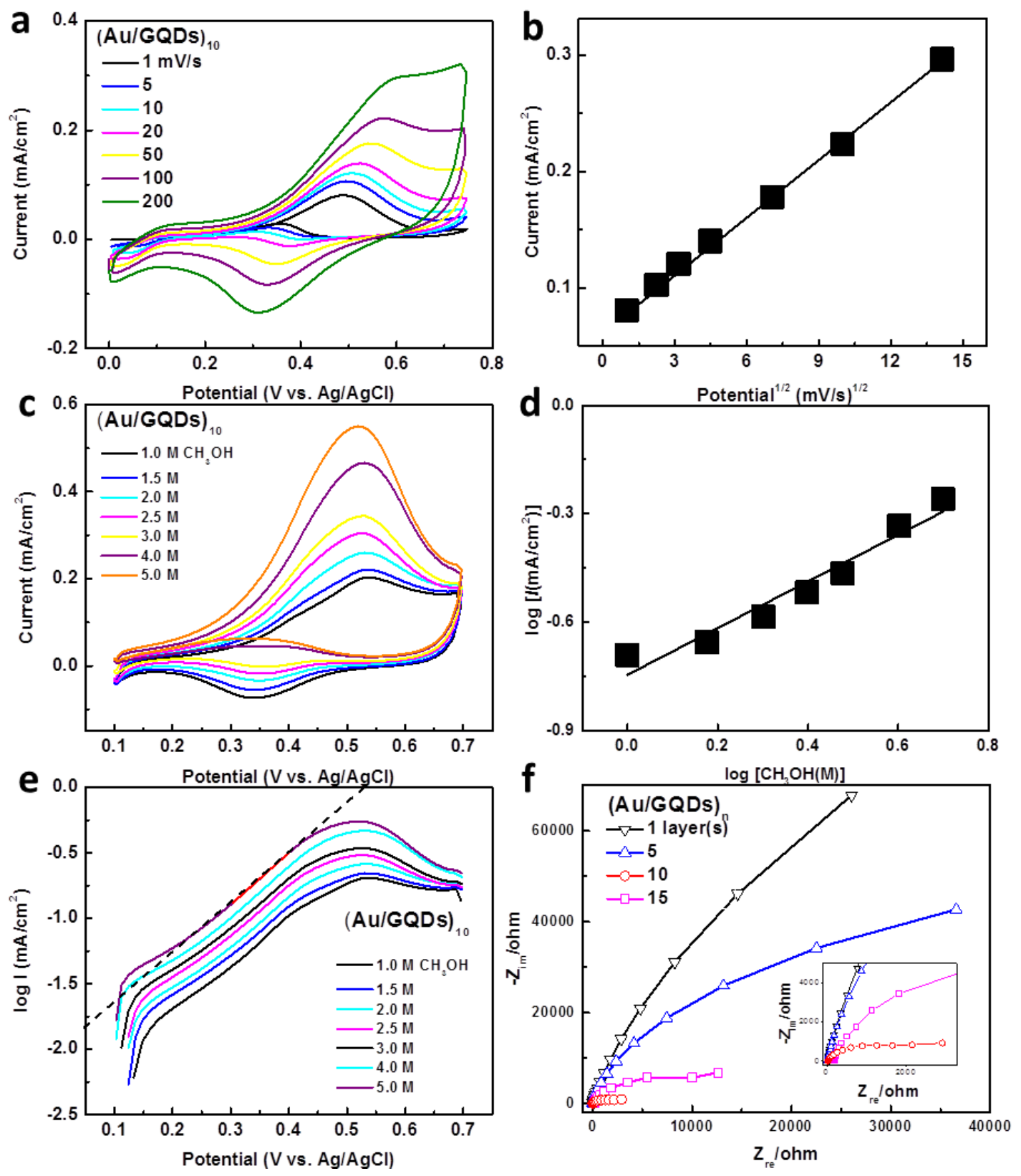

Figure 3-21. (a) $\mathrm{CV}$ results of (Au/GQDs) $)_{10}$ multilayer thin film measured in KOH solution $(0.10 \mathrm{M})$ with $1.0 \mathrm{M}$ methanol in a $\mathrm{N}_{2}$ atmosphere with varied scan rates, and (b) the corresponding linear correlation of anodic peak current as a 
function of the square root of scan rates. (c) CV results of (Au/GQDs) 10 multilayer thin film with different methanol concentrations, (d) the corresponding plots of $\log I$ ( $I$ is anodic peak current) vs. $\log \left(\mathrm{C}_{\mathrm{CH} \text { OH }}\right)$ with different methanol concentrations, and (e) Tafel curves with a linear fit. (f) Nyquist plots of impedance for (Au/GQDs) $)_{n}$ multilayer thin films measured in $\mathrm{KOH}$ solution $(0.10 \mathrm{M})$ with $\mathrm{CH}_{3} \mathrm{OH}(1.0 \mathrm{M})$ in $\mathrm{N}_{2}$ atmosphere at a scan rate of $50 \mathrm{mV} / \mathrm{s}$.

Electrocatalytic methanol oxidation process and mechanism over (Au/GQDs) $)_{10}$ multilayer films were further explored. As manifested in Figure 3-21(a \& b), CV results of $(\mathrm{Au} / \mathrm{GQDs})_{10}$ multilayer thin films under different scan rates indicated that the square root of scan rates can be linearly proportional to anodic peak currents, implying the electrocatalytic methanol oxidation process is controlled by a diffusion progress.(49) Moreover, as displayed in Figure 3-21(c \& d), anodic peak current (I) of $(\mathrm{Au} / \mathrm{GQDs})_{10}$ film increases with increasing the methanol concentration $\left(\mathrm{C}_{\mathrm{CH} 3 \mathrm{OH}}\right)$ and $\log I$ is proportional to $\log \left(\mathrm{C}_{\mathrm{CH} 3 \mathrm{OH}}\right)$, further corroborating the diffusion-controlled process. Contrarily, cathodic peak current decrease with increasing the concentration of methanol, which can be ascribed to the reduced reduction of Au oxide on the surface upon sweeping to negative potential.(49) It should be noted that the cathodic peak current tend to disappear when the methanol concentration is larger than $3.0 \mathrm{M}$, which might be due to the thick Au oxide coverage. Analogous results have also been observed in methanol oxidation over $(\mathrm{Au} / \mathrm{GR})_{10}$ multilayer films (Figure 3-22). The corresponding Tafel plots (Figure 3-21e) of (Au/GQDs) $)_{10}$ multilayer films for the methanol oxidation using different methanol concentrations at a scan rate of $50 \mathrm{mV} / \mathrm{s}$ were obtained. The linear correlation for $\log I$ as a function of potential are shown in the Tafel range, by which Tafel slope for (Au/GQDs) $)_{10}$ was determined to be 251.6 $\mathrm{mV} / \mathrm{dec}$ in the potential range of $0.3-0.4 \mathrm{~V}(v s . \mathrm{Ag} / \mathrm{AgCl})$, and the transfer coefficient was calculated to be 0.18 , indicating that the first charge transfer can be demonstrated 
as the rate-determining step.(193-195) The reaction orders of electrochemical methanol oxidation at fixed potentials can be obtained from the slope of the straight line fit (0.61) (Figure 3-23). The result suggested that kinetic parameters of methanol oxidation over $(\mathrm{Au} / \mathrm{GQDs})_{10}$ multilayer film remain unchanged in the Tafel ranges with increasing the concentration of methanol.(196)
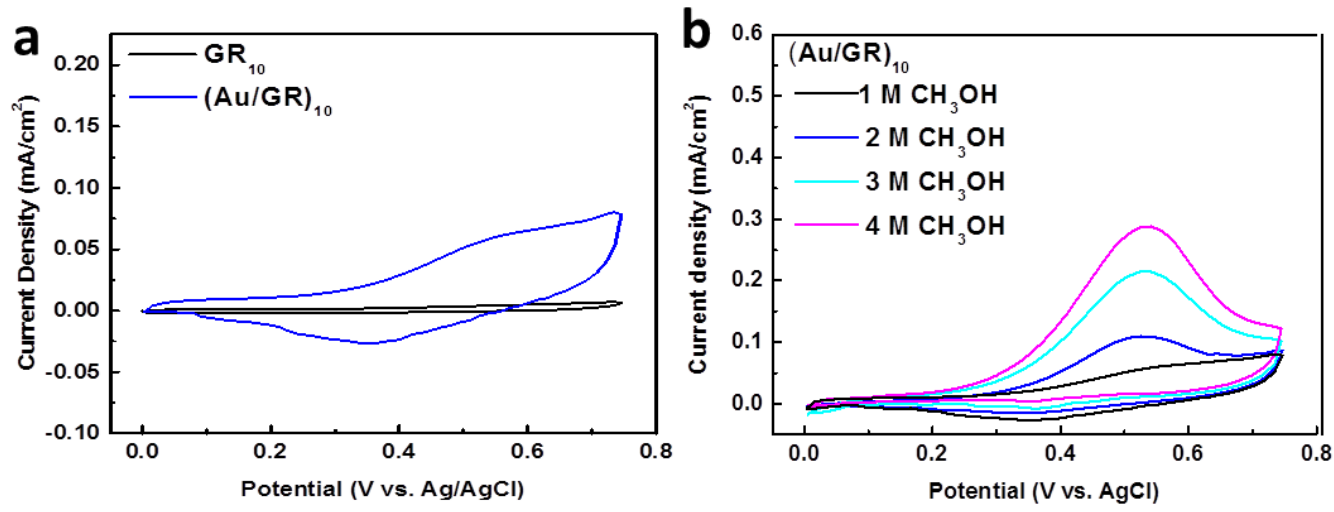

Figure 3-22. (a) $\mathrm{CV}$ results of $(\mathrm{GR})_{10}$ and $(\mathrm{Au} / \mathrm{GR})_{10}$ multilayer films measured in $\mathrm{KOH}$ solution $(0.10 \mathrm{M})$ with 1 methano $(\mathrm{l.0} \mathrm{M})$ in a $\mathrm{N}_{2}$ atmosphere under ambient conditions. (b) $\mathrm{CV}$ results of $(\mathrm{Au} / \mathrm{GR})_{10}$ multilayer thin film with different methanol concentrations.

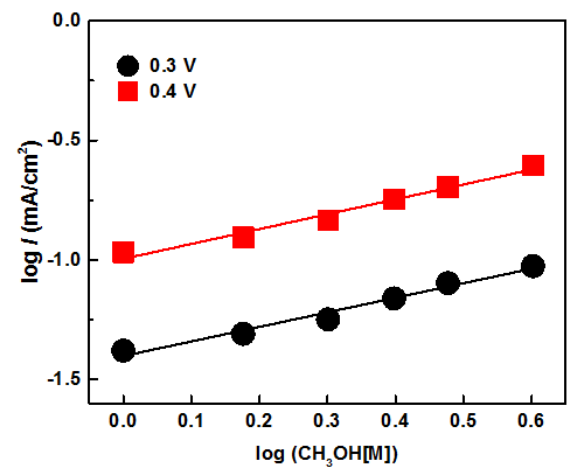

Figure 3-23. Plots of $\log (I)$ as a function of $\log \left(\mathrm{C}_{\mathrm{CH} \text { OH }}\right)$ at different potentials in Tafel range.

The kinetics and interfacial resistance of different multilayer thin films were explored by electrochemical impedance spectroscopy (EIS).(197,198) The interfacial charge transfer resistances of $(\mathrm{Au} / \mathrm{GQDs})_{\mathrm{n}}$ multilayer film electrode with varied 
assembly layer during the methanol oxidation reaction were measured. As reflected by the Nyquist plots of (Au/GQDs) $)_{n}$ multilayer films in Figure 3-21f, (Au/GQDs) ${ }_{10}$ multilayer film demonstrated the smallest semi-radius among other counterpart films, indicating the lowest interfacial charge transfer resistance and thus resulting in the optimal electrocatalytic activities which is in faithful agreement with selective catalytic reduction performances. Alternatively, it was revealed that (Au/GQDs) ${ }_{10}$ film demonstrated significantly lower charge transfer resistance compared with $(\mathrm{Ag} / \mathrm{GQDs})_{10}$ and (Pt/GQDs) $)_{10}$ films (Figure 3-24), indicating interfacial charge transfer resistance of $(\mathrm{M} / \mathrm{GQDs})_{\mathrm{n}}$ multilayer films can not only be finely tuned by the number of assembly layer but also category of metal NCs.

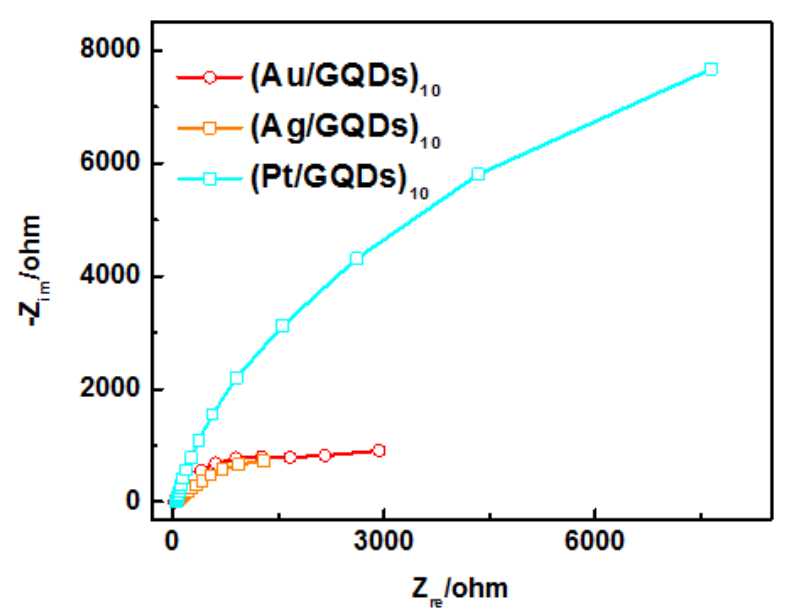

Figure 3-24. Nyquist plots of (M/GQDs $)_{10}(\mathrm{M}=\mathrm{Au}, \mathrm{Ag}, \mathrm{Pt})$ multilayer films measured in $\mathrm{KOH}$ solution $(0.10 \mathrm{M})$ with methanol $(1.0 \mathrm{M})$ in a $\mathrm{N}_{2}$ atmosphere (scan rate, $50 \mathrm{mV} / \mathrm{s}$ ).

\subsubsection{Photoelectrochemical catalytic performance of $(\mathrm{M} / \mathrm{GQDs})_{\mathrm{n}}(\mathrm{M}=\mathrm{Au}, \mathrm{Ag}$,}

\section{Pt) hybrid nanostructures}

Photoelectrochemical (PEC) measurements were further performed to explore the role of interfacial integration between metal NCs and GQDs, and meanwhile, to probe the separation of photoinduced electro-hole charge carriers under light 
irradiation. Figure 3-25 shows the PEC performance of (M/GQDs $)_{n}$ heterostructures. The transient photocurrent response of different samples under chopped light illumination (Figure 3-26) shows that concurrent deposition of Au NCs and GQDs via LbL assembly strategy is conducive to remarkably enhancing photocurrent of $(\mathrm{Au} / \mathrm{GQDs})_{10}$ heterostructure, from which the photocurrent density of (Au/GQDs) 10 is almost three-fold than that of (GQDs) ${ }_{10}$. The significantly improved photocurrent indicates a more effective separation of photogenerated electron-hole pairs over $(\mathrm{Au} / \mathrm{GQDs})_{10}$ binary nanostructure as compared with single counterparts. Consistently, Figure 3-26b shows LSV results exhibit analogous trends, thus confirming synergistic contribution of $\mathrm{Au} \mathrm{NCs}$ and GQDs for photocurrent generation.
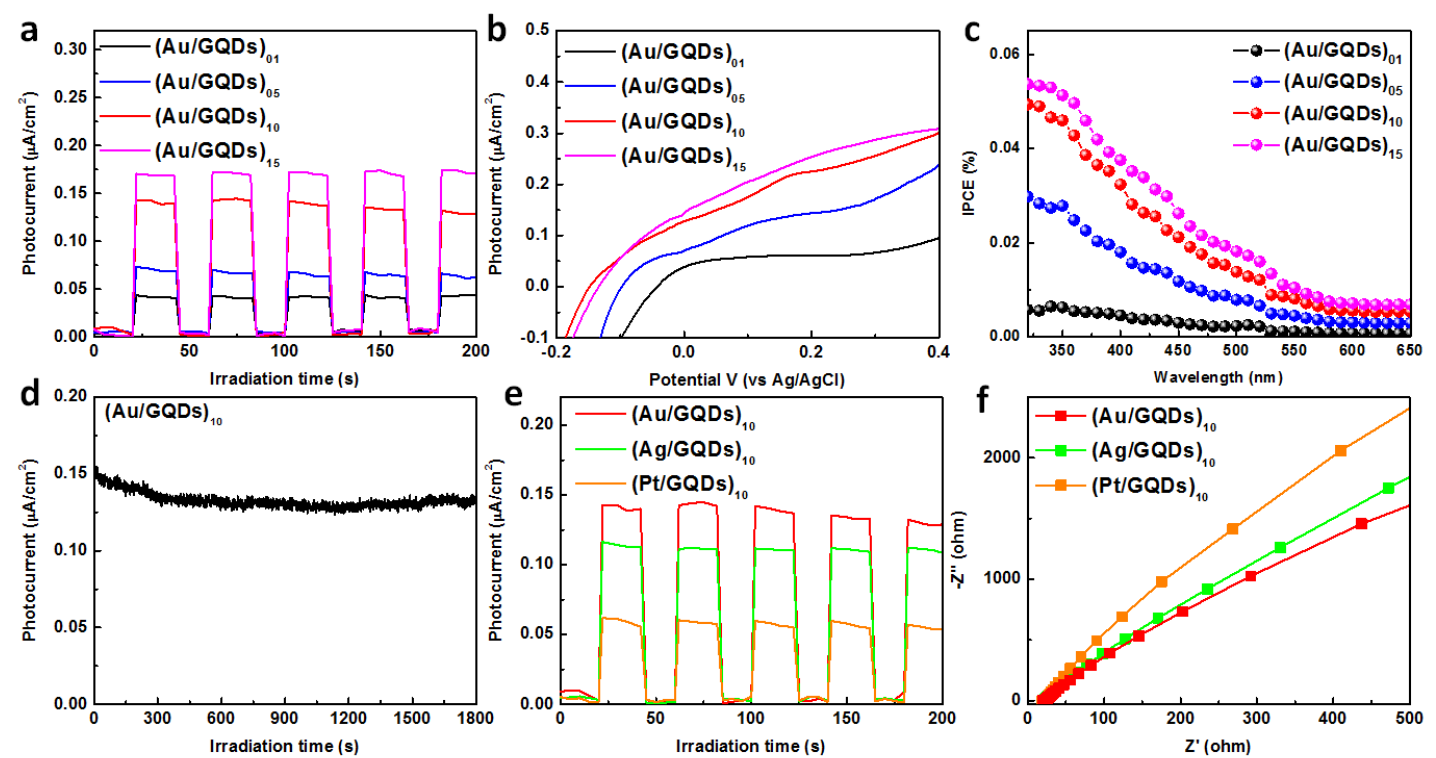

Figure 3-25 (a) The periodic on/off transient photocurrent response, and (b) linear-sweep voltammogram (LSV, scan rate: $1 \mathrm{mV} / \mathrm{s})$, and (c) IPCE spectra of $(\text { Au/GQDs })_{n}(n=1,5,10,15)$ heterostructures. (d) Photostability of (Au/GQDs) heterostructure. (e) Transient photocurrent responses, and (f) EIS Nyquist plots in low-frequency region of (M/GQDs) $)_{10}(\mathrm{M}=\mathrm{Au}, \mathrm{Ag}, \mathrm{Pt})$.

Electrochemical impedance spectroscopy (EIS) was further applied to evaluate the separation efficiency of photogenerated charge carriers.(84) As displayed in 
Figure 3-26c, EIS Nyquist plots of (Au/GQDs) $)_{10}$ exhibit substantially smaller semicircles in comparison with $(\mathrm{Au})_{10}$ and $(\mathrm{GQDs})_{10}$, implying much more efficient interfacial electron transfer imparted by the synergistic contribution of Au NCs and GQDs. Furthermore, Figure 3-25a shows the transient photocurrent response of $(\mathrm{Au} / \mathrm{GQDs})_{\mathrm{n}}(\mathrm{n}=1,5,10,15)$ heterostructure under intermittent simulated solar light illumination. Apparently, the photocurrent density of samples increases with the increasing layer (n) of the composite heterostructure. It can be observed that photocurrent of $(\mathrm{Au} / \mathrm{GQDs})_{\mathrm{n}}$ is increasing with the forward applied bias voltage under light illumination (Figure 3-25b), implying its n-type semiconductor performance.(199) Furthermore, EIS Nyquist plots of (Au/GQDs) $)_{n}$ heterostructure (Figrue 3-27a) shows the semicircles decrease with increasing the layer (n).
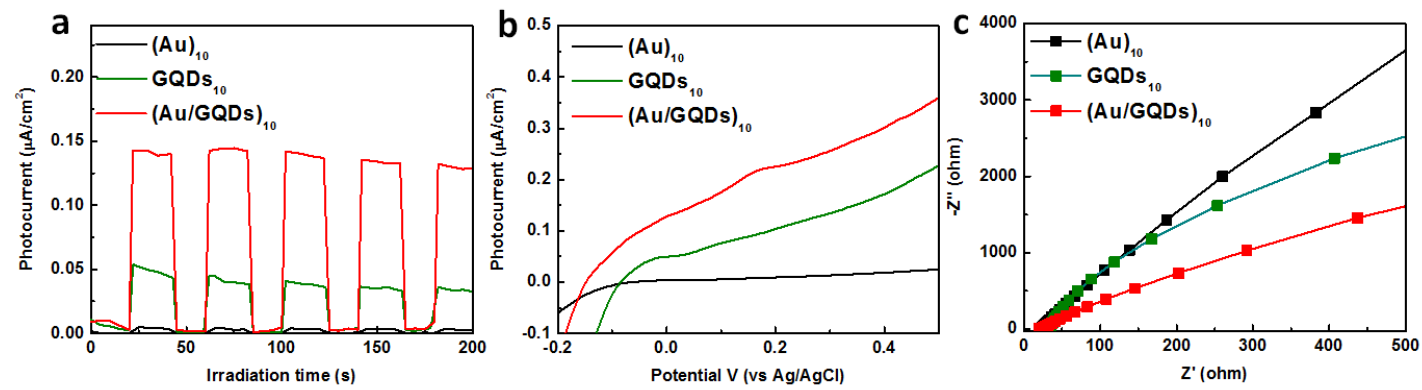

Figure 3-26. (a) The periodic on/off transient photocurrent response, and (b) linear-sweep voltammogram curves (LSV, scan rate: $1 \mathrm{mV} / \mathrm{s}$ ), and (c) electrochemical impedance spectroscopy (EIS) Nyquist plots in low-frequency region of $(\mathrm{Au})_{10},(\mathrm{GQDs})_{10}$, and $(\mathrm{Au} / \mathrm{GQDs})_{10}$ heterostructures.

Incident photo-to-current conversion efficiency (IPCE) measurements were further performed to reveal the underlying reasons for the generation of photocurrent versusincident light wavelength. As displayed in Figure 3-25c, IPCE of (Au/GQDs) nanotructure exhibit two pronounced peaks at around 350 and $510 \mathrm{~nm}$, thereby indicating the synergistive contributions of $\mathrm{Au}$ NCs and GQDs in generating photocurrent under imulated solar light irradiation. Notably, Photocurrent of 
$(\mathrm{Au} / \mathrm{GQDs})_{10}$ under continuous simulated solar light irradiation (Figure 3-25d) indicates the favorable photostability. Besides, PEC performances of (Ag/GQDs) 10 and $(\mathrm{Pt} / \mathrm{GQDs})_{10}$ nanostuctures were also probed (Figure 3-25e, f, and 3-27b). Figure 3-25e shows the transient photocurrent response of (Ag/GQDs) $)_{10}$ and (Pt/GQDs) $)_{10}$ multilayer films under intermittent simulated solar light illumination. It can also be observed that EIS Nyquist plots of (Ag/GQDs) 10 and (Pt/GQDs) $)_{10}$ is similar semicircles in comparison with (Au/GQDs) $)_{10}$ (Figure 3-25f), implying substantially efficient interfacial electron transfer with the synergistic contribution of $\mathrm{Ag}(\mathrm{Pt}) \mathrm{NPs}$ and GQDs. Therefore, PEC performances of (M/GQDs) ${ }_{\mathrm{n}}$ could be tuned by assembly cycle (n) and metal NCs (M) during LbL assembly process.
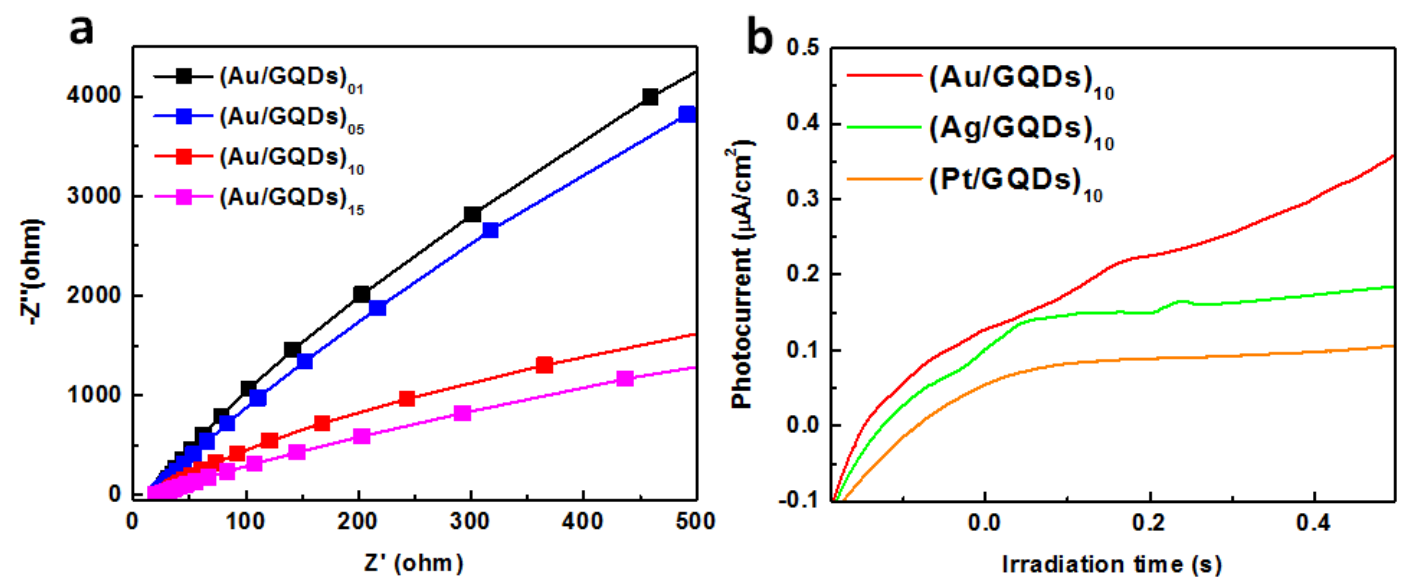

Figure 3-27. (a) EIS Nyquist curves in low-frequency region of (Au/GQDs) $(\mathrm{n}=1,5,10,15)$. (b) Linear-sweep voltammograms (LSV, scan rate: $1 \mathrm{mV} / \mathrm{s})$ of $(\mathrm{M} / \mathrm{GQDs})_{10}(\mathrm{M}=\mathrm{Au}, \mathrm{Ag}, \mathrm{Pt})$.

\subsubsection{Selective catalytic reduction, electrochemical and photoelectrochemical catalytic mechanisms}

Selective catalytic, electrocatalytic and PEC mechanisms of the (M/GQDs) ${ }_{n}$ heterostructure were depicted in Figure 3-28. For selective catalytic activities, the predominant reason accounting for the most remarkably enhanced catalytic activities of (M/GQDs) $)_{n}$ multilayer films can be ascribed to the multiple functionality of GQDs 
layer which facilitates the adsorption of 4-NP molecular owing to analogous structure, thus boosting the reaction kinetics, as well as preventing the agglomeration of metal NCs with their free electrons, and enhancing the stability of multilayer composite film (Figure 3-28a). Meanwhile, the unique electronic structure of the zigzag edges of graphene in GQDs nanostructure can interact with the terminal oxygen atoms of nitrophenol derivatives, thus weakening the $\mathrm{N}-\mathrm{O}$ bonds for effective reduction to occur.

As for the electrocatalytic methanol oxidation mechanism, two main factors influencing the highly efficient methanol oxidation performances of (M/GQDs) multilayer thin films can be attributed to the methanol diffusion and mass transfer in conjunction with electron transfer in the $\mathrm{LbL}$ assembled thin films by the following pathways, which concurrently dictate the fine balance of $(\mathrm{M} / \mathrm{GQDs})_{\mathrm{n}}$ multilayer thin films electrodes during the electrocatalytic process (Figure 3-28b). Therefore, the forward anodic peak current of $(\mathrm{M} / \mathrm{GQDs})_{\mathrm{n}}$ hybrid electrode began to decrease while the the layer $\mathrm{n}$ is more than 10 , possibly owing to the limited methanol diffusion in the electrode composite with increasing thickness, and to reduce mass and electron transfer to metal NCs.

$$
\begin{aligned}
& \mathrm{CH}_{3} \mathrm{OH}+\mathrm{Au}-\mathrm{OH}_{\mathrm{ad}}{ }^{(1-\lambda)-}+4 \mathrm{OH}^{-} \rightarrow \mathrm{Au}+\mathrm{HCOO}^{-}+4 \mathrm{H}_{2} \mathrm{O}+(4-\lambda) \mathrm{e}^{-} \\
& \mathrm{Au}+\mathrm{HCOO}^{-} \rightarrow \mathrm{Au}-\mathrm{HCOO}_{\mathrm{ad}}{ }^{(1-\lambda)^{-}}+\lambda \mathrm{e}^{-} \\
& \mathrm{Au}-\mathrm{HCOO}_{\mathrm{ad}}{ }^{(1-\lambda)-}+4 \mathrm{OH}^{-} \rightarrow \mathrm{Au}+\mathrm{CO}_{2}+\mathrm{H}_{2} \mathrm{O}+(2-\lambda) \mathrm{e}^{-}
\end{aligned}
$$

With regard to PEC water splitting performance, based on the Kohn-Sham molecular orbital theory, electron transitions from the occupied densities to the unoccupied densities are triggered by GQDs under light illumination, thereby imparting GQDs with a distinct Highest Occupied Molecular Orbital (HOMO)Lowest Unoccupied Molecular Orbital (LUMO) gap which performs as a 
semiconductor with a narrow band gap (2.4-2.6 eV).(107,196) Accordingly, under simulated solar light irradiation, electrons can be photoexcited from HOMO to LUMO over GQDs to generate electron-hole pairs (Figure 3-28c). Moreover, it has been well-established that "hot electrons" provided from plasmon excitation of $\mathrm{Au}$ NCs under simulated solar light illumination could transmit to the LUMO of GQDs, therefore increasing the density of photo-induced electrons in reaction system. The photogenerated electrons can be rapidly transferred to the FTO substrate, then further transmit to the counter electrode to undergo the reaction with water for hydrogen evolution. Meanwhile, photogenerated holes produced on the HOMO of GQDs and "hot holes" over Au NCs can react with water for Oxygen evolution, inducing the efficient separation of electron-hole pairs and achieving photocurrent. 


\section{a Selective catalytic mechanism}

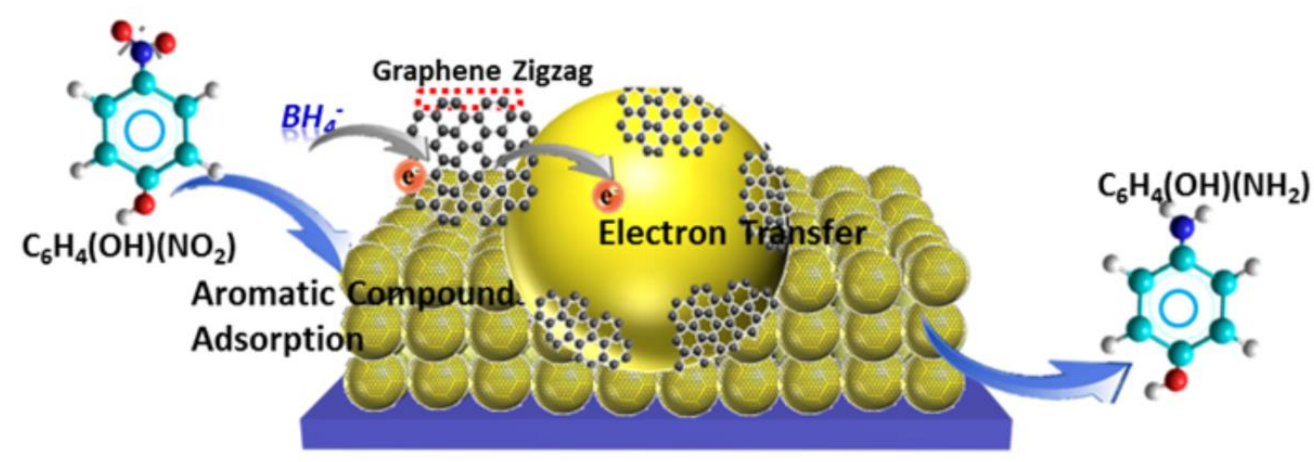

\section{b Electrocatalytic mechanism}

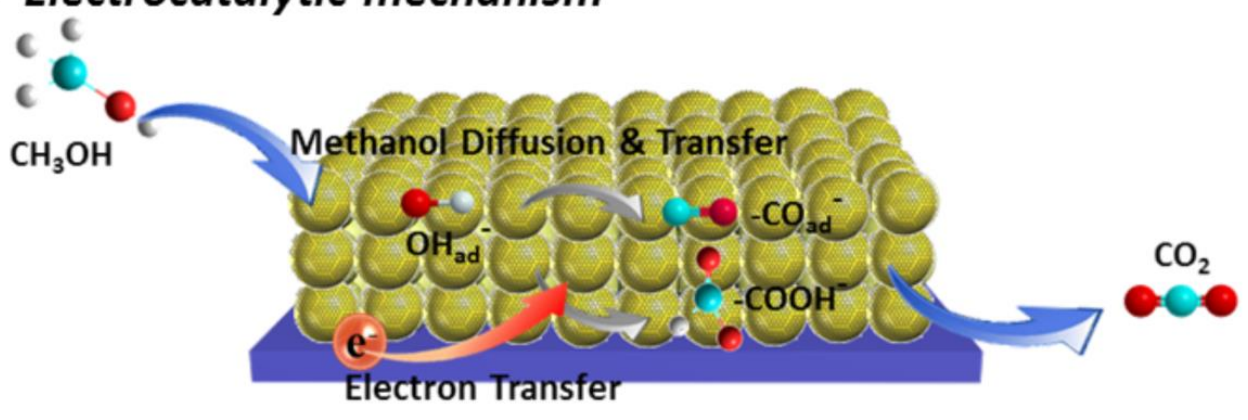

C Photoelectrocatalytic mechanism

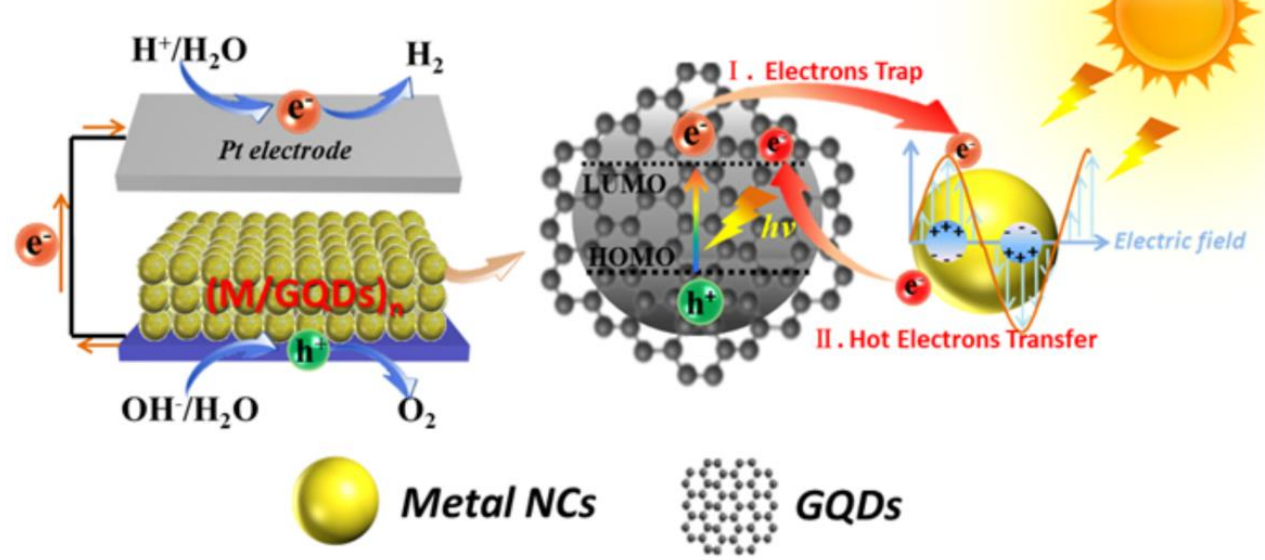

Figure 3-28. Scheme of depicting the (a) selective catalytic, (b) electrocatalytic, and (c) PEC mechanisms of (M/GQDs) $)_{n}$ heterostructures.

\subsection{Summary}

In summary, an efficient yet simple LbL self-assembly approach has been developed to rationally and judiciously fabricate (M/GQDs) $(\mathrm{M}=\mathrm{Au}, \mathrm{Ag}, \mathrm{Pt})$ multilayer composite thin films based on pronounced electrostatic attractive 
interaction, by which tailor-made metal NCs and GQDs serving as predominant building blocks were alternately and closely assembled in an intimate alternate stacking resulting in unique 0D/0D hybrid multilayered nanoarchitectures. The welldefined (M/GQDs) $)_{n}$ multilayer composite thin films demonstrated highly efficient selective catalytic performances for reducing aromatic nitro compounds, and electrocatalytic methanol oxidation under ambient conditions and solar lightirradiated PEC water splitting. More significantly, selective catalytic reduction, and electrocatalytic methanol oxidation and PEC performances of (M/GQDs) $)_{n}$ multilayer composite films are capable to be finely controlled by the number of assembly layer and category of metal NCs, among which (Au/GQDs) $)_{n}$ exhibited the optimal versatile and tunable catalytic and electrocatalytic activities. It is anticipated that the general concept presented in our work could provide a new platform for rational design and construction of various functional $0 \mathrm{D} / 0 \mathrm{D}$ nanostructures for catalysis and/or chemical-to-energy conversion. 


\section{Chapter 4 Layer-by-Layer of nitrogen-doped}

\section{graphene quantum dots monolayer decorated one-}

\section{dimensional semiconductor nanoarchitectures for}

\section{solar-driven water splitting ${ }^{1}$}

\subsection{Introduction}

Diverse strategies have been developed to fabricate various $1 \mathrm{D} \mathrm{ZnO}$-based hybrid nanostructures for PEC water splitting under simulated solar light or visible light irradiation, such as noble metal deposition (e.g., Au, Ag, and Pt),(200-203) transition metal ions doping or non-metal elemental atoms doping,(204-207) sensitization with narrow-band-gap semiconductors (e.g., CdS, CdSe, CdTe, and PbS quantum dots),(208) or formation of nanostructured heterostructures with secondary semiconductors.(209) Despite these endeavors, constructing well-defined 1D ZnObased composite heterostructures with efficient light absorption especially in visible region along with efficient separation of photogenerated electron-hole pairs continues to be challenging.

As one kind of carbon dots (CDs), graphene quantum dots (GQDs) composed of strong $\mathrm{sp}^{2}$-bonded carbon possess better crystallinity structure than its cousins, which have garnered increasing research interest because of its analogous semiconductor properties with tuneable bandgap.(14-22,100,210) In spite of the fascinating physicalco-chemical properties of GQDs, reports on rational design of GQDs/semiconductor

\footnotetext{
${ }^{1}$ Reproduced from [Zeng Z., Xiao F.-X., Gui X., Wang R., Liu B., Tan T. Y. T., Journal Materials Chemistry A, 2016, 4, 16383-16393.] with permission from The Royal Society of Chemistry 
nanocomposites are still rare. Note that synthetic approaches for fabricating GQDs/semiconductor nanocomposites in previous work are relatively complicated, and environmentally-unfriendly, and tunable control over GQDs deposition is still far from satisfactory.(211) Moreover, intimate integration of GQDs and semiconductors at the nanoscale level via conventional approach is hard to achieve, which is of paramount importance to interfacial charge carrier transport. On the other hand, it has been well-established that electrical conductivity of GQDs and charge-transfer as well as electrolyte-electrode interactions can be significantly improved by nitrogen doping in graphene frame, $(212,213)$ which is in favor of catalyzing water-splitting under visible light irradiation. Although nitrogen-doped graphene have been explored in various fields, such as fuel cells,(214) lithium ion batteries,(215) and supercapacitors,(216) until now, the construction of well-defined NGQDs/semiconductor heterostructures through a facile, green, and easily accessible synthetic strategy for PEC hydrogen production has not yet be reported.

Layer-by-Layer (LbL) assembly technique, as one of promising bottom-up fabrication strategies, demonstrates remarkable advantages compared with conventional approaches in terms of simplicity and versatility, which furnishes tunable control over micro-structure, thickness, and composition of the assemblies.(83-86) Particularly, intrinsic negatively charged surface and hydrophility of N-GQDs renders it a suitable building block for LbL assembly on the hydrophilic surface of $\mathrm{ZnO} \mathrm{NWs}$ based on electrostatic interaction, thereby resulting in a tailorable nano-architecture. Herein, a facile and green LbL assembly approach has been developed to construct highly ordered N-GQDs/ZnO NWs heterostructures based on substantial electrostatic interaction, in which N-GQDs prepared by a facile synthetic strategy, was used as a predominant building block for LbL assembly on the 
ZnO NWs framework. Moreover, it was found that the LbL-assembled N-GQDs/ZnO NWs heterostructures exhibit significantly enhanced PEC water splitting performances under both simulated solar and visible light illumination. Furthermore, the deposition amount of N-GQDs on the scaffold of $\mathrm{ZnO}$ NWs can be easily tuned by assembly layer. The contributing role of N-GQDs was clearly defined and the PEC mechanism of $\mathrm{N}-\mathrm{GQDs} / \mathrm{ZnO} \mathrm{NWs}$ heterostructures was specifically discussed. It is hoped that this work can offer a new synthetic strategy for the rational fabrication of N-GQDs/semiconductor nanoarchitectures and, more significantly, afford new insights for the potential applications of N-GQDs in the field of photocatalysis or PEC water splitting.

\subsection{Experimental section}

\subsubsection{Fabrication of nitrogen-doped GQDs, ZnO NWs, TiO2 NRs}

\section{Synthesis of N-GQDs}

N-GQDs was prepared by using GQDs and ammonium nitrate as precursors.(48) $100 \mathrm{mg}$ GQDs and $100 \mathrm{mg}$ ammonium nitrate were dissolved in $10 \mathrm{~mL}$ of ethanol and the mixed solution was stirred for $20 \mathrm{~min}$. Then, the solution temperature was increased to $60{ }^{\circ} \mathrm{C}$ to evaporate ethanol. After that, the mixture can be put into a furnace for calcination at $350{ }^{\circ} \mathrm{C}$ for $1.5 \mathrm{~h}$. Finally, the sample was dissolved in DI $\mathrm{H}_{2} \mathrm{O}$ under 30 min sonication and dialyzed (MWCO, 12-14 KD, Spectra/Por) for 3 days to remove residual ammonium and nitrate ions, and dried in an over at $100{ }^{\circ} \mathrm{C}$ for $12 \mathrm{~h}$.

\section{Synthesis of ZnO NWs and TiO2 NRs}

Fluorine-doped tin oxide (FTO) substrates were washed by using a piranha solution $\left(3: 198 \% \mathrm{H}_{2} \mathrm{SO}_{4} / 30 \% \mathrm{H}_{2} \mathrm{O}_{2}\right)$ for 30 min, washed with $\mathrm{DI}_{2} \mathrm{O}$, and dried 
with a gentle stream of $\mathrm{N}_{2} .110 \mathrm{mg}$ zinc acetate was added to ethanol solution to obtain $5 \mathrm{mM}$ zinc acetate solution. 5-8 drops of the prepared zinc acetate solution was then dropped onto the FTO substrate with conducting layer facing up. After about 25 $\mathrm{s}$, the piece of FTO was rinsed in pure ethanol solution and immediately taken out and put in an electric oven at $100{ }^{\circ} \mathrm{C}$ for drying. This process was repeated for 4 times, and thus the FTO with $\mathrm{ZnO}$ seeds was obtained by calcinating at $350{ }^{\circ} \mathrm{C}$ in air for 30 $\min$.

The growth solution was prepared by adding $100 \mathrm{~mL}$ of $0.05 \mathrm{M}$ of methenamine into $100 \mathrm{~mL}$ of $0.05 \mathrm{M}$ zinc nitrate solution. A piece of FTO coated with $\mathrm{ZnO}$ seeds was immersed into the growth solution, containing $25 \mathrm{mM} \mathrm{Zn}\left(\mathrm{NO}_{3}\right)_{2}$ and $25 \mathrm{mM}$ methenamine and kept in an electric oven at $95^{\circ} \mathrm{C}$ for $3 \mathrm{~h}$. Finally, the as-prepared $\mathrm{ZnO}$ NWs were calcinated at $400{ }^{\circ} \mathrm{C}$ in air for $1 \mathrm{~h}$.

$\mathrm{TiO}_{2}$ nanorod arrays $\left(\mathrm{TiO}_{2} \mathrm{NRs}\right)$ on $\mathrm{FTO}$ substrate were prepared by a hydrothermal method based on our previous work. ${ }^{63,64}$ Typically, FTO substrates were washed by using a piranha solution $\left(3: 198 \% \mathrm{H}_{2} \mathrm{SO}_{4} / 30 \% \mathrm{H}_{2} \mathrm{O}_{2}\right)$ for $30 \mathrm{~min}$, washed with DI $\mathrm{H}_{2} \mathrm{O}$, and then dried with $\mathrm{N}_{2}$. The samples was fist synthesized by adding titanium butoxide ( $97 \%$, Aldrich, $0.45 \mathrm{~mL}$ ) to a solution including $\mathrm{HCl}(15$ $\mathrm{mL})$ and $\mathrm{DI} \mathrm{H}_{2} \mathrm{O}(15 \mathrm{~mL})$, and the solution was continuously stirred for 10 min until the mixture can be observed to be clear. Then, the resultant solution was put into a Teflon-liner stainless autoclave $(50 \mathrm{~mL})$ with the FTO substrates. Hydrothermal method was carried out at $150{ }^{\circ} \mathrm{C}$ for $12 \mathrm{~h}$. Afterwards, the FTO substrate were washed with $\mathrm{DI} \mathrm{H}_{2} \mathrm{O}$ and then dried in the air. 


\subsubsection{LbL assembly of N-GQDs/ZnO NWs (N-GQDs/TiO2 NRs) and GQDs/ZnO NWs (GQDs/TiO2 NRs) heterostructures}

The detailed assembly procedure is illustrated in Figure 4-1. The substrate was firstly immersed into polyethylenimine (PEI) solution $(1.0 \mathrm{mg} / \mathrm{mL}, \mathrm{pH}=7.23)$ for 10 min, then washed 3 times with DI $\mathrm{H}_{2} \mathrm{O}$, and dried with $\mathrm{N}_{2}$. Afterwards, the substrate was dipped into N-GQDs or GQDs aqueous suspension $(1.0 \mathrm{mg} / \mathrm{mL}, \mathrm{pH}=6.89)$ for 10 min, washed with $\mathrm{DI} \mathrm{H}_{2} \mathrm{O}$, and dried by $\mathrm{N}_{2}$. The above treatment as one whole was regarded as one single assembly layer. Multilayered deposition of N-GQDs or GQDs on $\mathrm{ZnO}$ NWs was achieved by repeating the dipping cycle. It should be pointed out that different concentrations (from 0.2 to $1.5 \mathrm{mg} / \mathrm{mL}$,) and different assembly layers (from 1 to 20 layers,) of GQDs were utilized in fabricating GQDs/ZnO NWs heterostructures, and the results indicate that the optimal parameter is $1.0 \mathrm{mg} / \mathrm{mL}$ GQDs aqueous suspension with one assembly layer. Thus, N-GQDs aqueous suspension was prepared based on $1.0 \mathrm{mg} / \mathrm{mL}$ GQDs aqueous solution and one assembly layer of N-GQDs was deposited on the $\mathrm{ZnO}$ NWs. Finally, the LbLassembled N-GQDs/ZnO and GQDs/ZnO NWs heterostructures were calcined in an argon atmosphere furnace at $400{ }^{\circ} \mathrm{C}$ for $1 \mathrm{~h}$. GQDs/TiO 2 NRs and N-GQDs/TiO 2 NRs heterostructures were fabricated with similar method as mentioned above. 


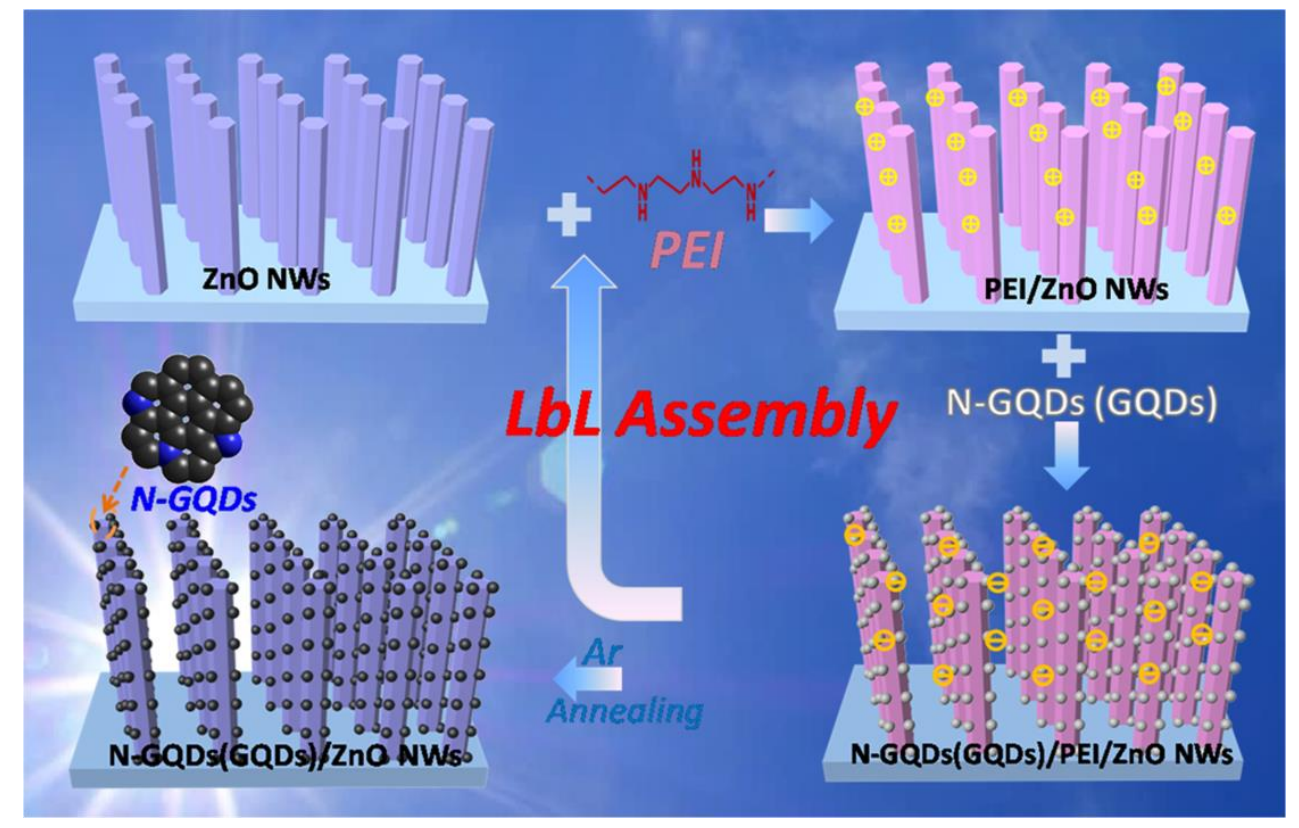

Figure 4-1 Schematic illustration for LbL assembly of N-GQDs (or GQDs) decorated $\mathrm{ZnO}$ nanowires arrays (N-GQDs (or GQDs)/ZnO NWs) heterostructures.

\subsubsection{Samples characterizations}

Scanning electron microscopy (SEM) results can be achieved with field emission scanning electron microscopy (JEOL, JSM-6700F). Transmission electron microscopy (TEM) images were measured by using a JEOL model JEM2010 (200 kV). X-ray photo electron spectroscopy (XPS) data were collected through ESCALAB 250 photoelectron spectrometer (Thermo Fisher Scientific). UV-vis spectra were obtained with a Shimadzu UV2501 spectrophotometer. The fluorescence properties were studied using a Fluoromax-4, Horiba Jobin Yvon Spectrofluorometer with a photon-counting detection system to detect fluorescence emission. Fourier Transform Infrared Spectroscopy (FTIR) was achieved by using a Digilab FTS 3100 instrument.

\subsubsection{PEC water splitting performances measurements}

PEC water splitting test were achieved in a three-electrode quartz cell with electrochemical workstation (CHI 66D) in which $\mathrm{Ag} / \mathrm{AgCl}$ electrode was used as a 
reference electrode and $\mathrm{Pt}$ sheet was employed as a counter electrode, and the samples with the same area $\left(15.9 \mathrm{~mm}^{2}\right)$, provided by a mask with a diameter of 4.5 mm, was employed as the working electrodes. $\mathrm{Na}_{2} \mathrm{SO}_{4}$ solution $(0.5 \mathrm{M})$ was used as the electrolyte. $100 \mathrm{~mW} \mathrm{~cm}^{-2}$ of light source was provided by a $300 \mathrm{~W}$ Xenon arc lamp. The incident photon-to-current conversion efficiency (IPCE) spectra of the samples were measured by using monochromatic light, which is carried out with a Xenon light irradiated under a Cornerstone monochromator. The signal of photocurrent was achieved by a Merlin lock-in radiometry. The amount of evolved gas was detected by a gas chromatograph (Agilent 7890A GC with TCD detector; carrier gas: $\mathrm{N}_{2}$ ). Typically, a inflow of $\mathrm{N}_{2}$ was continuously bubbled into the electrolyte $\left(0.5 \mathrm{M} \mathrm{Na}_{2} \mathrm{SO}_{4}\right.$ solution) for half hour $30 \mathrm{~min}$ to remove traces of $\mathrm{O}_{2}$ and $\mathrm{H}_{2}$ in the gas collection system. During the measurement of gaseous product, the simulated solar light source (or visible light source) was provided by a $300 \mathrm{~W}$ Xenon arc lamp, and the gas was manually injected into GC at an interval of $30 \mathrm{~min}$.

\subsection{Results and discussion}

\subsubsection{Synthesis and characterization of N-GQDs}
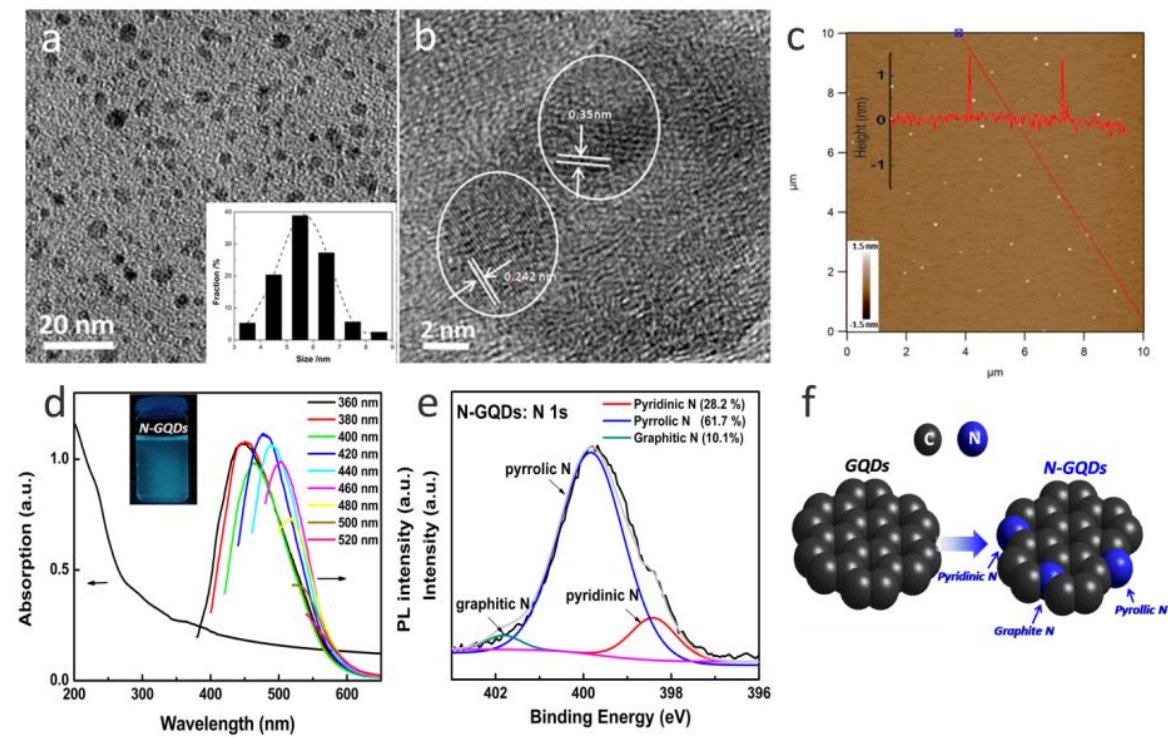
Figure 4-2 (a) TEM image of N-GQDs with size distribution histogram in the inset, (b) high-resolution TEM (HRTEM) image of N-GQDs, (c) AFM image of N-GQDs with corresponding height profile in the inset, (d) UV-vis absorption spectrum and photoluminescence spectra of N-GQDs with photoexcited digital graph (365 nm UV light) in the inset, (e) high-resolution N 1s XPS spectrum of N-GQDs, and (f) schematic illustration of N-GQDs with different types of doped nitrogen atoms.

A transmission electron microscopy (TEM) image (Figure 4-2a) depicts that NGQDs possess uniform size ranging from 3 to $9 \mathrm{~nm}$ with mean particle size of $5.8 \mathrm{~nm}$ (inset of Figure 4-2a). Figure 4-2b shows the high-resolution TEM (HRTEM) of NGQDs in which lattice-fringe spacing were determined to be $c a .0 .242$ and $0.35 \mathrm{~nm}$, which correspond well to (1120) and (002) crystal planes of graphite, $(19,24,44,45,217)$ respectively. Undoped GQDs also exhibit uniform size of around $5.6 \mathrm{~nm}$ (Figure 4-3a), implying the particle size of GQDs was barely changed with nitrogen doping. Moreover, as shown in Figure 4-2c, atomic force microscope (AFM) was utilized to probe the morphology and height of N-GQDs, of which the height profile was determined to be $c a \cdot 1.0-1.5 \mathrm{~nm}$, strongly suggesting that N-GQDs consisted of 1-2 graphene layers inherited from GQDs precursor. This conclusion can be corroborated by the AFM result of GQDs (Figure 4-2b) which exhibits analogous topographic height profile ranging from 0.7 to $1.2 \mathrm{~nm}$. 

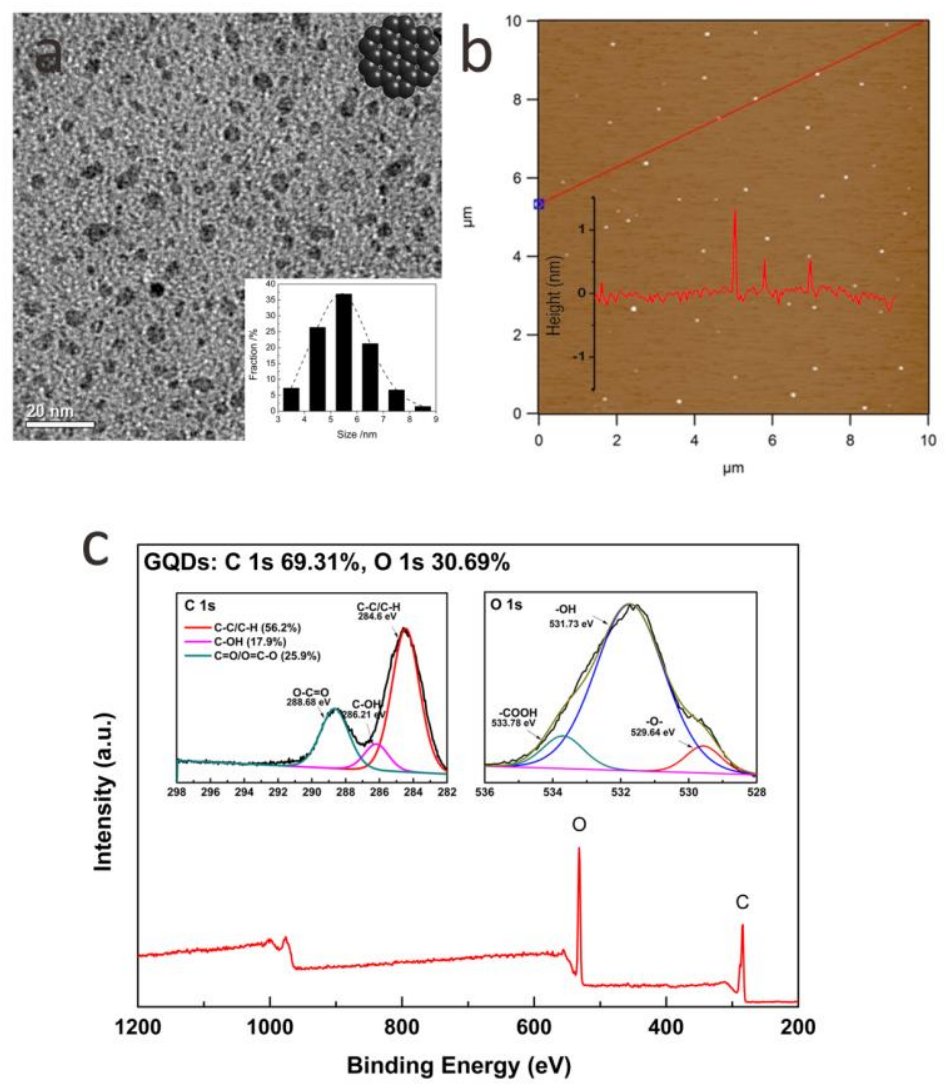

Figure 4-3 (a) TEM images with size distribution histogram in the inset, (b) AFM image of GQDs, and (c) survey and high-resolution $C 1$ and $O$ 1s spectra of GQDs.

Noteworthily, although UV-vis absorption spectrum of N-GQDs (Figure 4-2d) displays similar light absorption to GQDs (Figure 4-4) with two characteristic absorption bands at 240 and $350 \mathrm{~nm}$ attributed the quantum confinement effect, $\mathrm{N}$ GQDs exhibit substantially enhanced absorption in visible region which can be ascribed to the nitrogen doping. Furthermore, as displayed in the inset of Figure 4-2d, an obvious blue luminescence was observed over N-GQDs when it was irradiated with UV light $(365 \mathrm{~nm})$ which is in agreement with the photoluminescence feature of N-GQDs.(217) The PL emission band of N-GQDs can be tuned by exciting light wavelength ranging from 360 to $520 \mathrm{~nm}$, indicating an unique optoelectronic property due to large defects amount and high conductivity of nitrogen-doping of GQDs.(45) 
Fourier transform infrared spectroscopy (FTIR) characterization (Figure 4-5a) depicts the typical peak of GQDs at $1712 \mathrm{~cm}^{-1}$, corresponding to carbonyl and carboxylic acid groups. However, the peak decreases after nitrogen doping, suggesting that carbonyl and carboxylic functional groups of GQDs are involved in the intermolecular dehydrolysis reaction between neighboring carbonyl/carboxyl and amine groups to form pyrrolic $N$ in GQDs.(45) Notably, a new peak at $1577 \mathrm{~cm}^{-1}$ was clearly observed in the FTIR spectrum of N-GQDs, which is attributed to $\mathrm{C}=\mathrm{N}$ bond in N-GQDs, thus indicating nitrogen has been successfully doped in the GQDs frame.(212) As shown in Figure 4-5b, N-GQDs exhibit larger $\mathrm{I}_{\mathrm{D}} / \mathrm{I}_{\mathrm{G}}$ in the Raman results ratio than GQDs which is mainly due to the introduction of defects and formation of different bond lengths between $\mathrm{C}-\mathrm{C}$ and $\mathrm{C}-\mathrm{N}$ bonds afforded by nitrogen doping. $(46,47)$

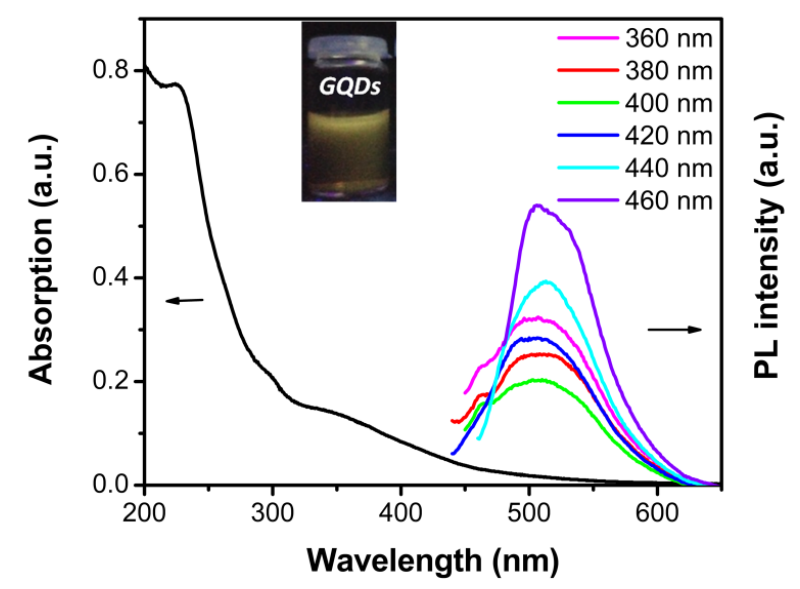

Figure 4-4 UV-vis absorption spectrum and PL spectra of GQDs solution with photoexcited digital graph $(360 \mathrm{~nm}$ UV light) in the inset. 

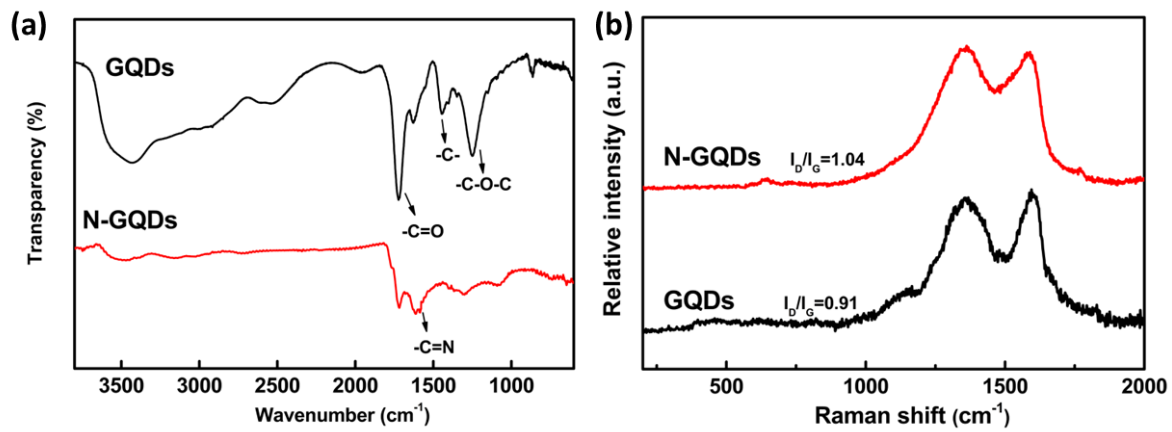

Figure 4-5 FT-IR spectra (a) and Raman spectra (b) of GQDs and N-GQDs.

Survey X-ray photoelectron spectroscopy (XPS) spectrum of N-GQDs is displayed in Figure 4-6a, which reveals that N-GQDs consists mainly of C, N, and O core-elements. More specifically, high-resolution C 1s spectrum of N-GQDs (Figure 4-6b) can be deconvoluted to four Gaussian peaks located at 284.6, 286.13, 287.2 and $288.08 \mathrm{eV}$, corresponding to the $\mathrm{C}-\mathrm{C} / \mathrm{C}-\mathrm{H}, \mathrm{C}-\mathrm{OH}, \mathrm{C}-\mathrm{N}$, and $\mathrm{O}=\mathrm{C}-\mathrm{OH}$ bonds, respectively.(16,48,218) Consistently, high-resolution $\mathrm{N}$ 1s spectrum of N-GQDs, as shown in Figure 4-2e, can be deconvoluted predominantly to three peaks at 398.3, 399.8 and $401.8 \mathrm{eV}$, which correspond to pyridine, pyrrolic and graphitic nitrogen atoms, respectively. $(16,218)$ Moreover, percentage of nitrogen atoms doped in the $\mathrm{N}$ GQDs was determined to be $c a .5 .4$ at. \% based on XPS result, which is comparable to values reported by other synthetic approaches, such as annealing with $\mathrm{NH}_{3}$ atmosphere $(46,48)$ or microware plasma-assisted chemical vapor deposition method.(49,50) The result indicates that our synthetic method is effective in preparing $\mathrm{N}$-GQDs with favorable nitrogen doping level. In addition, atomic ratios of $\mathrm{C} 1 \mathrm{~s}$ to $\mathrm{O}$ 1s $(\mathrm{C} / \mathrm{O})$ in N-GQDs and GQDs were determined to be 3.28 and 1.24, indicating GQDs were partially reducted during the nitrogen doping process. Consequently, XPS results suggest that nitrogen-doping and partial reduction of GQDs occurred simultaneously, which would facilitate restoring the electron conductivity of GQDs. 
For clarification, different types of C-N bonds in N-GQDs were depicted in Figure 42f.
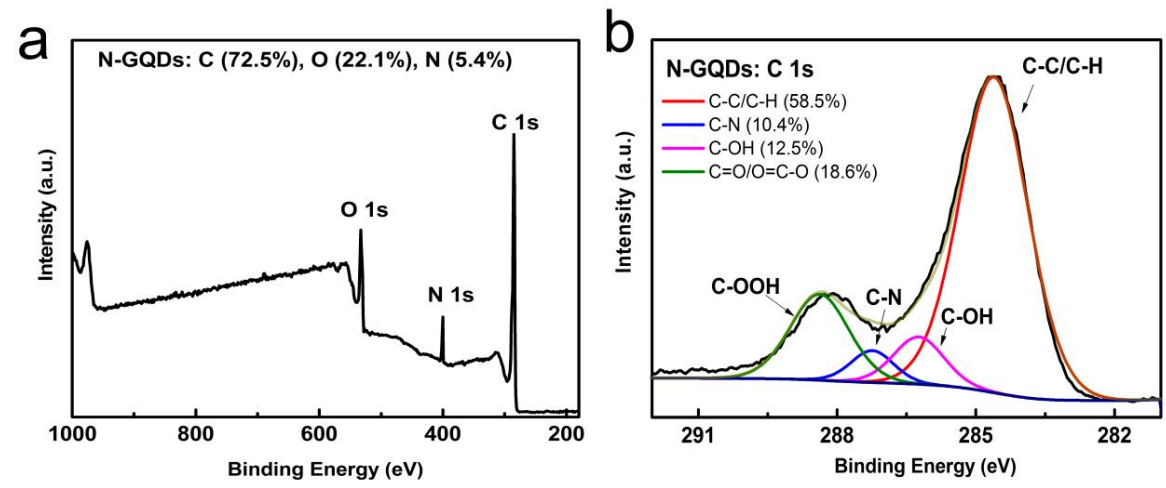

Figure 4-6 (a) Survey and (b) high resolution C 1s spectra of N-GQDs.

\subsubsection{LbL assembly of N-GQDs/ZnO NWs heterostructures}

Figure 4-1 depicts the process for LbL assembly of N-GQDs (or GQDs)/ZnO NWs heterostructure. Specifically, single-crystalline ZnO NWs grown vertically from fluorine-doped tin oxide (FTO) substrate were firstly fabricated by using a hydrothermal method. Subsequently, ZnO NWs scaffold after calcination was modified with polyethylenimine (PEI) which affords a positively charged surface. PEI has the unique feature of the presence of amine groups which endows it with high affinity to the hydrophilic ZnO NWs surface.(51) Based on which, negatively charged N-GQDs or GQDs (Figure 4-7) can be spontaneously and uniformly assembled on the $\mathrm{ZnO}$ NWs substrate via substantial electrostatic interaction, thereby giving rise to well-defined N-GQDs/ZnO (or GQDs/ZnO) NWs heterostructure with one deposition cycle of N-GQDs (or GQDs). Then, by the same way, the thus-attained N-GQDs/ZnO (or GQDs/ZnO) NWs heterostructure was re-treated with PEI and assembled with NGQDs (or GQDs), by which deposition amount of N-GQDs or GQDs on ZnO NWs was tuned by assembly cycle. Analogous to $\mathrm{ZnO}$ NWs, other $1 \mathrm{D}$ semiconductors such as $\mathrm{TiO}_{2}$ nanorod (NRs) can also be modified with N-GQDs or GQDs through 
the same LbL assembly strategy to fabricate $\mathrm{N}-\mathrm{GQDs}$ (or GQDs)/TiO $\mathrm{T}_{2} \mathrm{NRs}$ heterostructures. Finally, these LbL-assembled N-GQDs (or GQDs)/1D semiconductor ( $\mathrm{ZnO} \mathrm{NWs}$ or $\left.\mathrm{TiO}_{2} \mathrm{NRs}\right)$ nanoarchitectures were calcined in an argon atmosphere to completely remove PEI moiety and, simultaneously, to achieve reduction of N-GQDs (or GQDs), which is beneficial for restoring electron conductivity of N-GQDs (or GQDs) and achieving more intimate interfacial contact between N-GQDs (or GQDs) component and 1D semiconductors scaffold.

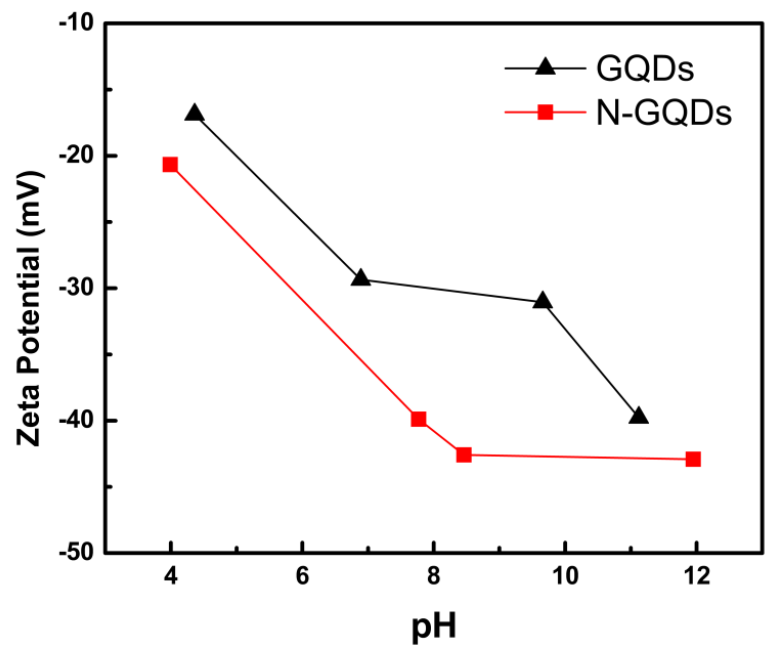

Figure 4-7 Zeta potentials of GQDs and N-GQDs aqueous solutions $(1.0 \mathrm{mg} / \mathrm{mL})$ versus pH value.
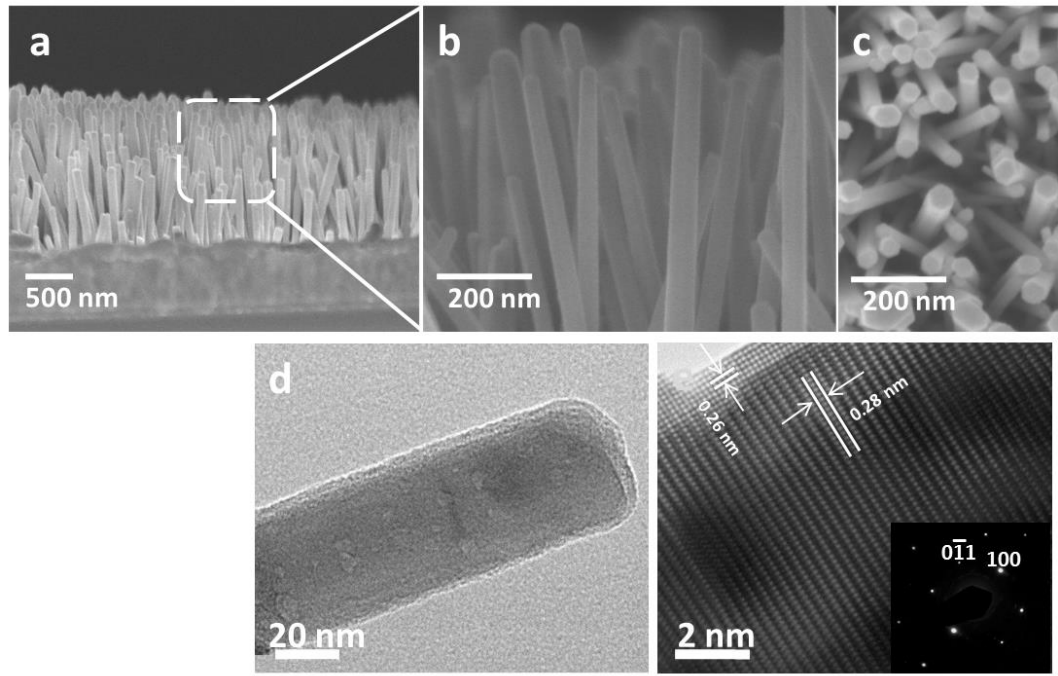

Figure 4-8 (a \& b) Cross-sectional, (c) top-view FESEM images, (d) TEM, and (e) HRTEM image (with a SAED pattern in the inset) of ZnO NWs. 

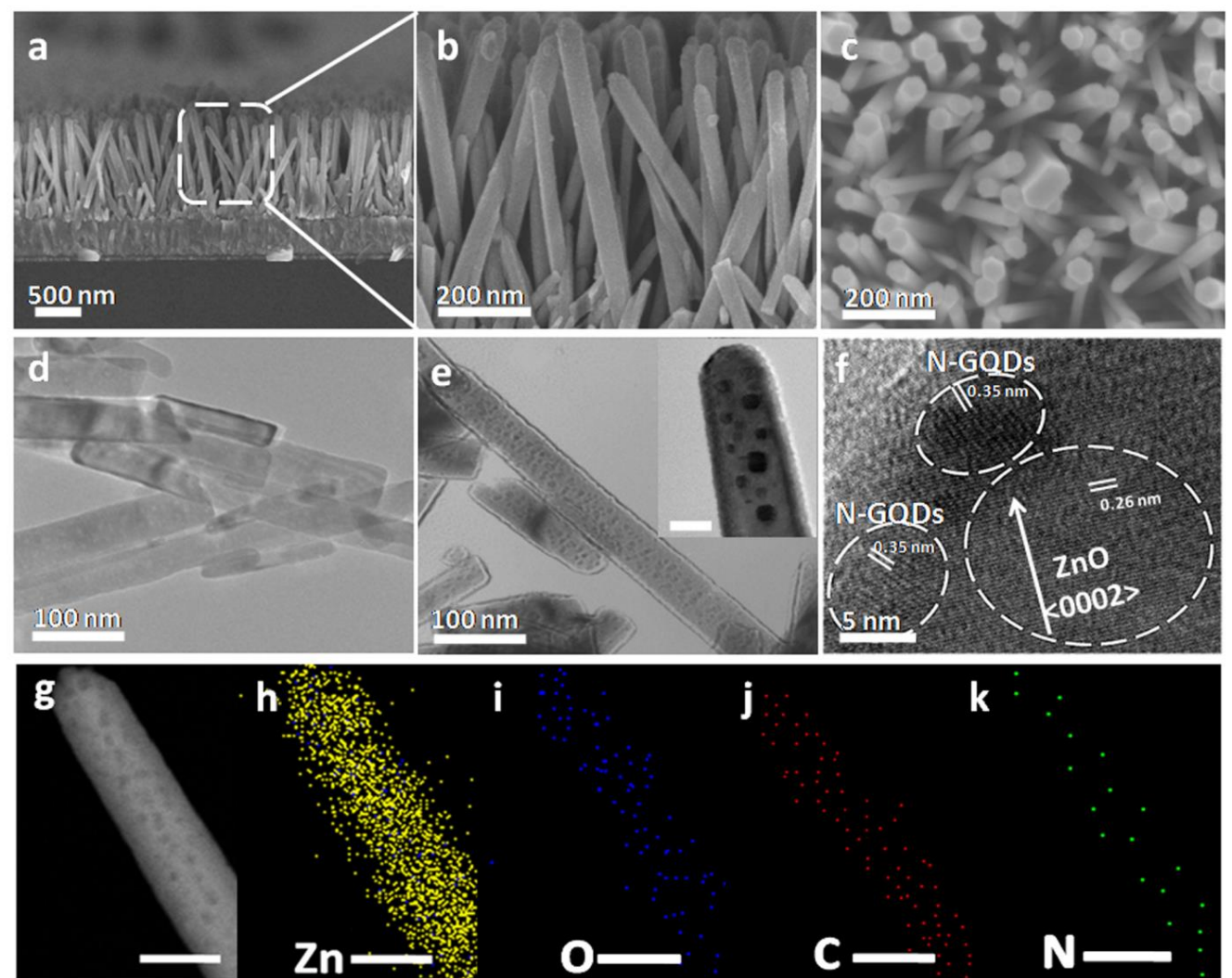

i

k

Figure 4-9 Cross-sectional (a \& b) and (c) top-view FESEM images of $\mathrm{N}$ GQDs/ZnO NWs heterostructure (one assembly layer), (d) TEM image of ZnO NWs, (e) TEM image of N-GQDs/ZnO NWs heterostructure with highmagnification image in the inset (scale bar: $20 \mathrm{~nm}$ ), (f) HRTEM image of $\mathrm{N}$ GQDs/ZnO NWs heterostructure, and (g) dark field-STEM image with corresponding EDS mapping spectra from (h) to (k) (the scale bar is $50 \mathrm{~nm}$ )

Field emission scanning electron microscopy (FESEM) was utilized to explore the morphologies of different samples. Cross-sectional FESEM image in Figure 4-8a shows that ZnO NWs grow vertically from FTO substrate with a thickness of $c a .1 .5$ $\mu \mathrm{m}$ and high-magnification FESEM image in Figure 4-8b exhibits ZnO NWs with an average diameter of $40 \mathrm{~nm}$. Panoramic FESEM image (Figure 4-8c) shows that $\mathrm{ZnO}$ NWs substrate is virtually composed of nanowire with regular hexagonal crosssection and flat top surface. As reflected in Figure 4-9 (a and b), N-GQDs/ZnO NWs heterostructure (one assembly layer) exhibits much rougher surface than blank $\mathrm{ZnO}$ NWs, indicating N-GQDs were uniformly coated on the ZnO NWs surface. Top-view FESEM image of $\mathrm{N}-\mathrm{GQD} / \mathrm{ZnO} \mathrm{NWs}$ heterostructure is analogous to blank $\mathrm{ZnO}$ 
NWs, implying the morphology of ZnO NWs was not altered with uniform deposition of N-GQDs.

Transmission electron microscopy (TEM) was used to verify the morphologies of the samples. As shown in Figure 4-9d and 4-8d, pristine $\mathrm{ZnO}$ NWs exhibit a smooth surface which agrees well with FESEM image. As revealed in the HRTEM result of $\mathrm{ZnO} \mathrm{NWs}$ (Figure 4-8e), lattice fringes were determined to be 0.28 and 0.26 $\mathrm{nm}$, which are attributable to the (100) and (011) crystal planes of hexagonal wurtzite $\mathrm{ZnO} .(52)$ This indicates that $\mathrm{ZnO} \mathrm{NWs}$ grow primarily along the (100) crystallographic plane which is in line with selected area electron diffraction result (inserted image in Figure 4-8e). When N-GQDs were assembled on the ZnO NWs substrate, it was found that the morphology of N-GQDs/ZnO NWs heterostructure (Figure 4-9e) is similar to pristine $\mathrm{ZnO} \mathrm{NWs}$ other than the appearance of various black spots on the surface, which is due to the deposition of N-GQDs with small particle size (ca. $5.8 \mathrm{~nm}$ ). Particularly, as displayed in Figure 4-9e (inset), TEM image of a single nanowire apparently revealed rougher surface of $\mathrm{N}-\mathrm{GQD} / \mathrm{ZnO}$ NWs heterostructure than blank ZnO NWs, indicating N-GQDs have indeed been closely attached to the surface of $\mathrm{ZnO} \mathrm{NWs}$ afforded by LbL assembly. The components of N-GQDs and $\mathrm{ZnO}$ NWs in composite heterostructure can be ascertained by HRTEM image (Figure 4-9f), and elemental mapping confirmed the coexistence of $\mathrm{C}$ and $\mathrm{N}$ on the $\mathrm{ZnO}$ NWs matrix in a homogeneous distribution (Figure 4-9h-k). In addition, uniform distribution of GQDs decorated with $\mathrm{ZnO}$ NWs can also be achieved by the same LbL assembly strategy (Figure 4-10). 


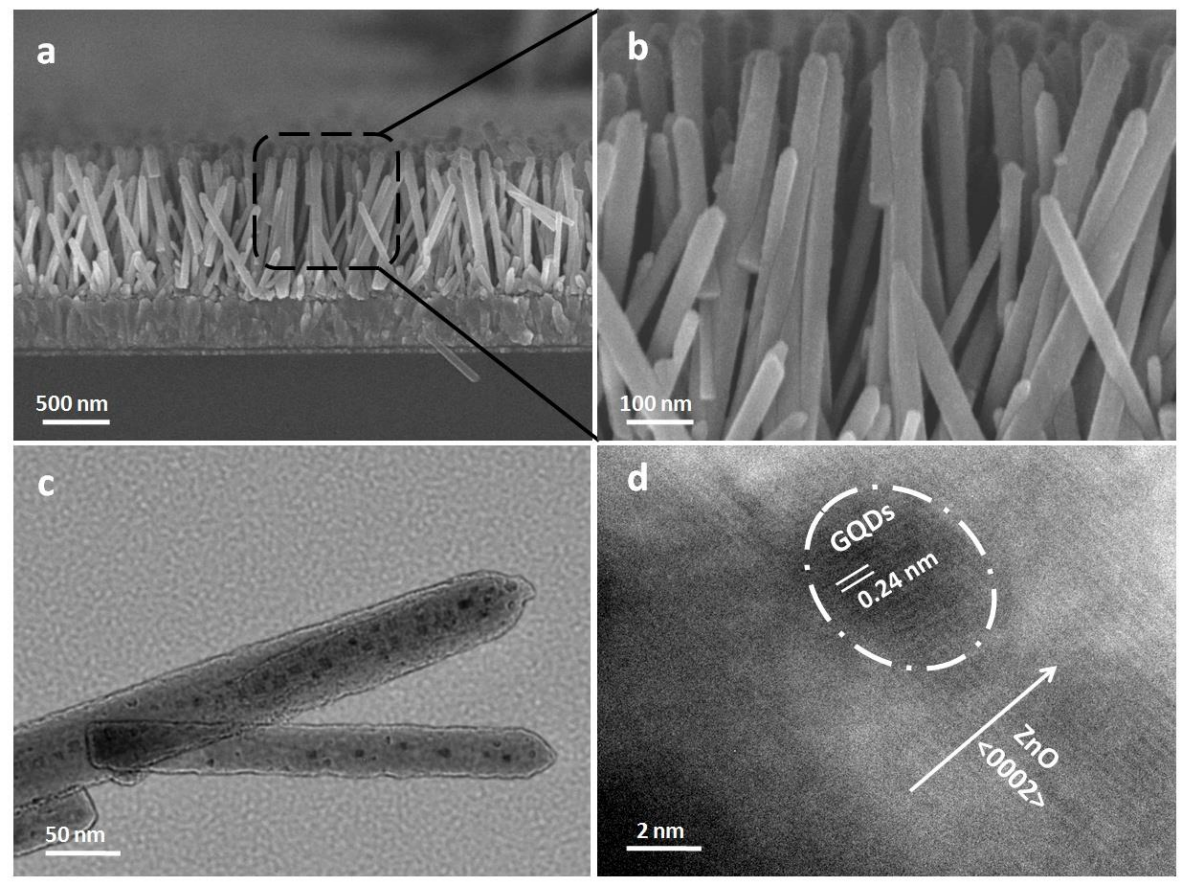

Figure 4-10(a \& b) Cross-sectional FESEM images of GQDs/ZnO NWs heterostructure (one assembly layer), (c) TEM and (d) HRTEM images of GQDs/ZnO NWs heterostructure (one assembly layer).
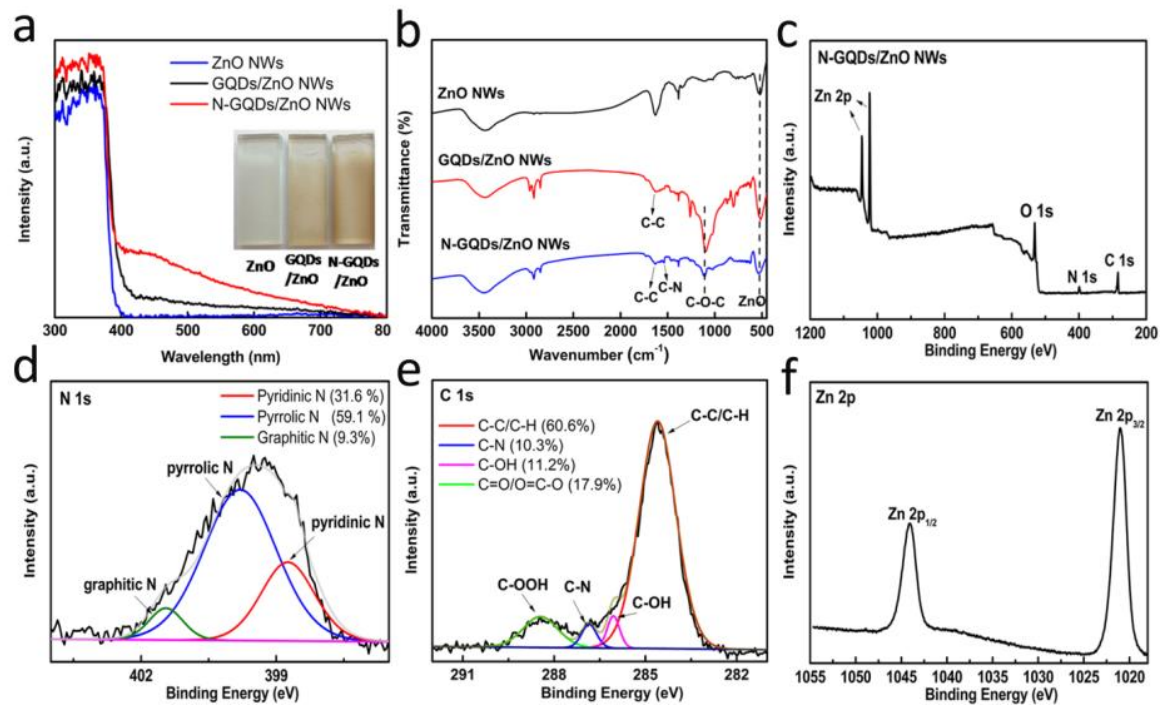

Figure 4-11 (a) UV-vis diffuse reflectance spectra (DRS) and (b) FTIR results of ZnO NWs, GQDs/ZnO NWs (one assembly layer) and N-GQDs/ZnO NWs heterostructure (one assembly layer), (c) Survey XPS spectrum, and highresolution (d) $\mathrm{N}$ 1s, (e) $\mathrm{C} 1 \mathrm{~s}$ and (f) $\mathrm{Zn} 2 \mathrm{p}$ spectra of $\mathrm{N}-\mathrm{GQDs} / \mathrm{ZnO} \mathrm{NWs}$ heterostructure (one assembly layer).

UV-vis diffuse reflectance spectra can be utilized to explore the optical properties of the $\mathrm{ZnO}$ composites. As displayed in Figure 4-11a, a substantial 
absorption peak in UV region for different samples was observed which is ascribed to the bandgap excitation of $\mathrm{ZnO}$. Moreover, it was found that, $\mathrm{N}-\mathrm{GQD} / \mathrm{ZnO} \mathrm{NWs}$ heterostructure with one assembly layer show distinct absorption band edge from blank $\mathrm{ZnO}$ NWs especially in visible region ranging from $400 \mathrm{~nm}$ to $800 \mathrm{~nm}$. Notably, DRS result of $\mathrm{ZnO}$ NWs exhibits no absorption above $400 \mathrm{~nm}$ which agrees with bandgap of $\mathrm{ZnO}$, while pronounced absorption in the same wavelength region was clearly seen for $\mathrm{N}-\mathrm{GQD} / \mathrm{ZnO} \mathrm{NW}$ s heterostructures, highlighting the pivotal role of N-GQDs as photo-sensitizers. Furthermore, it should be emphasized that NGQDs/ZnO NWs heterostructures demonstrate significantly enhanced light absorption in visible region as compared with GQDs/ZnO NWs counterpart with the same assembly layer, which highlights the contributing role of nitrogen doping in boosting light absorption of GQDs. In addition, as shown in Figure 4-12, bandgap energies of different samples was roughly determined to be around $2.85,2.90$, and $3.18 \mathrm{eV}$ corresponding to N-GQDs/ZnO NWs, GQDs/ZnO NWs and blank ZnO NWs, respectively. Therefore, DRS result suggests that uniform deposition of N-GQDs on $\mathrm{ZnO} \mathrm{NWs}$ is beneficial for enhancing light harvest of $\mathrm{ZnO}$ NWs.

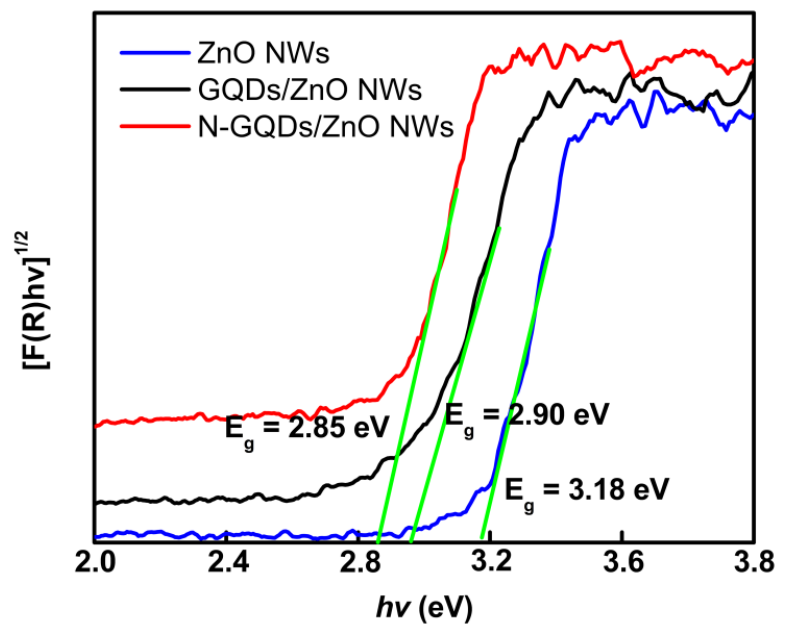

Figure 4-12. Plots of transformed Kubelka-Munk function versus the energy of light for ZnO NWs, GQDs/ZnO NWs and N-GQDs/ZnO NWs heterostructures (one assembly layer). 
FTIR spectra were utilized to further unveil the structure of different samples. As displayed in Figure 4-11b various carbonyl and carboxyl functional groups were observed in the FTIR spectrum of GQDs/ZnO NWs heterostructures. Apart from these peaks, the peak at $1577 \mathrm{~cm}^{-1}$ corresponding to N-GQDs arose and, meanwhile, peak intensity of some other functional groups (C-O-C stretching vibration mode) and $\mathrm{Zn}-\mathrm{O}$ group concurrently decreased in the FTIR spectrum of $\mathrm{N}-\mathrm{GQD} / \mathrm{ZnO} \mathrm{NWs}$ heterostructure as compared with FTIR results of $\mathrm{ZnO}$ NWs and GQDs/ZnO NWs counterparts. The decreased intensity of these characteristic peaks suggests that interaction between $\mathrm{N}-\mathrm{GQDs}$ and $\mathrm{ZnO} \mathrm{NWs}$ is different from that between GQDs and ZnO NWs.
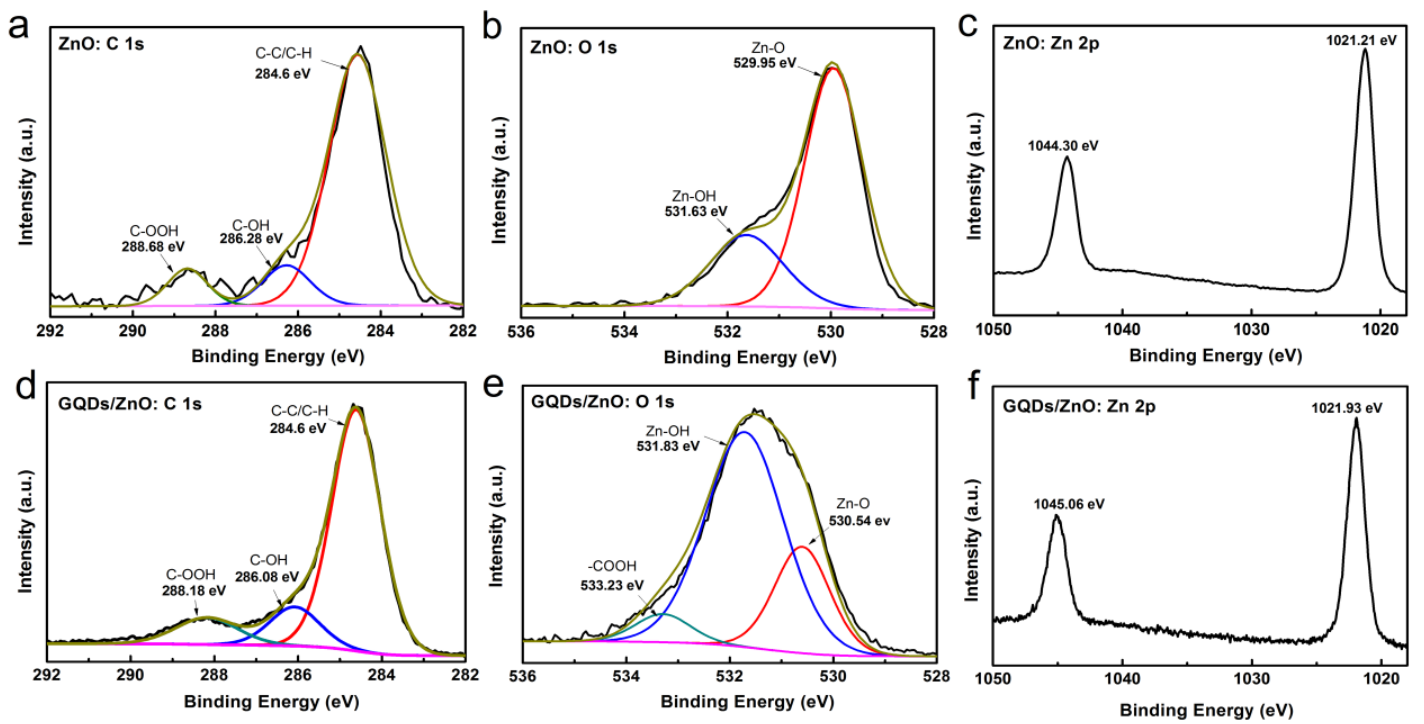

Figure 4-13. High-resolution (a) $\mathrm{C} \mathrm{1s}$, (b) $\mathrm{O}$ 1s and (c) $\mathrm{Zn} 2 \mathrm{p}$ spectra of $\mathrm{ZnO}$ NWs. High-resolution (d) C 1s, (e) $\mathrm{O}$ 1s and (f) Zn 2p spectra of GQDs/ZnO NWs heterostructure (one assembly layer).

Survey XPS curves of $\mathrm{ZnO}$ NWs, GQDs/ZnO NWs heterostructure, and NGQDs/ZnO NWs heterostructure were performed, as displayed in Figure 4-11 (c-f) \& 4-13. Survey XPS spectrum of N-GQDs/ZnO NWs heterostructure mainly consists of $\mathrm{C}, \mathrm{N}, \mathrm{O}$ and $\mathrm{Zn}$ core-elements, indicating successful deposition of N-GQDs on the ZnO NWs. More specifically, as displayed in Figure 4-11 (d \& e), high-resolution N 
1s and $\mathrm{C}$ 1s spectra of N-GQDs/ZnO NWs heterostructure can be deconvoluted to several Gaussian peaks corresponding well to different types of functional groups, and the results are consistent with high-resolution $\mathrm{N}$ 1s and C 1s spectra of N-GQDs (Figure 4-2 \& 4-6). Furthermore, atomic ratio of $\mathrm{C} 1 \mathrm{~s}$ to $\mathrm{N}$ 1s $(\mathrm{C} / \mathrm{N})$ in $\mathrm{N}-\mathrm{GQD} / \mathrm{ZnO}$ NWs heterostructure was determined to be $c a .13 .8 \%$. Thus, the results indicate that N-GQDs have been successfully assembled on the ZnO NWs framework. In addition, high-resolution Zn 2p spectrum (Figure 4-11f) for composite heterostructure shows binding energies of $1044.3 \mathrm{eV}$, and $1021.5 \mathrm{eV}$ for $\mathrm{Zn} 2 \mathrm{p}_{1 / 2}$ and $\mathrm{Zn} 2 \mathrm{p}_{3 / 2}$, which unambiguously points to elemental chemical state of $\mathrm{Zn}^{2+}$ and suggests that the deposition of N-GQDs did not modify the chemical state of $\mathrm{ZnO}$ substrate. ${ }^{(206)}$ Figure 4-14 shows the X-ray diffraction (XRD) patterns of different samples. Based on XRD result of ZnO NWs, six diffraction peaks including (100), (002), (001), (102), (110) and (103), were clearly identified, which correspond to wurtzite ZnO.(53) Moreover, it was found that diffraction peaks of $\mathrm{ZnO}$ were retained in the XRD pattern of $\mathrm{N}$ GQDs (or GQDs)/ZnO NWs heterostructures, indicating N-GQDs (or GQDs) modification did not affect the crystalline structure of $\mathrm{ZnO}$ which is in line with HRTEM (Figure 4-9) and XPS results (Figure 4-11). Notably, no diffraction peaks of GQDs were observed in the composite nanostructures and this can be ascribed to low deposition amount of N-GQDs (or GQDs). 


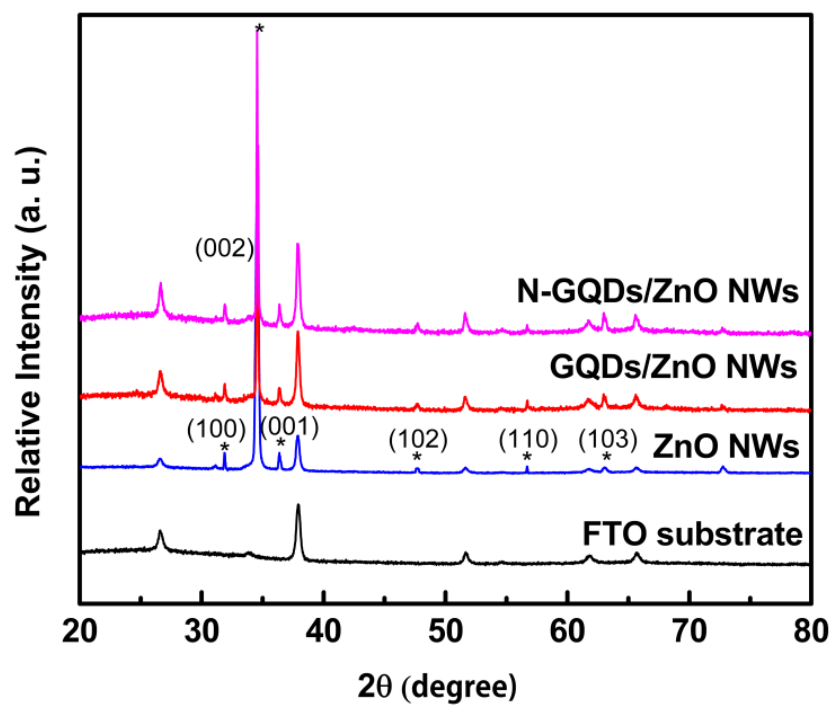

Figure 4-14 XRD patterns of ZnO NWs, GQDs/ZnO NWs heterostructure (one assembly layer) and $\mathrm{N}$-GQDs/ZnO NWs heterostructure (one assembly layer).

\subsubsection{Photoelectrochemical water splitting performances}
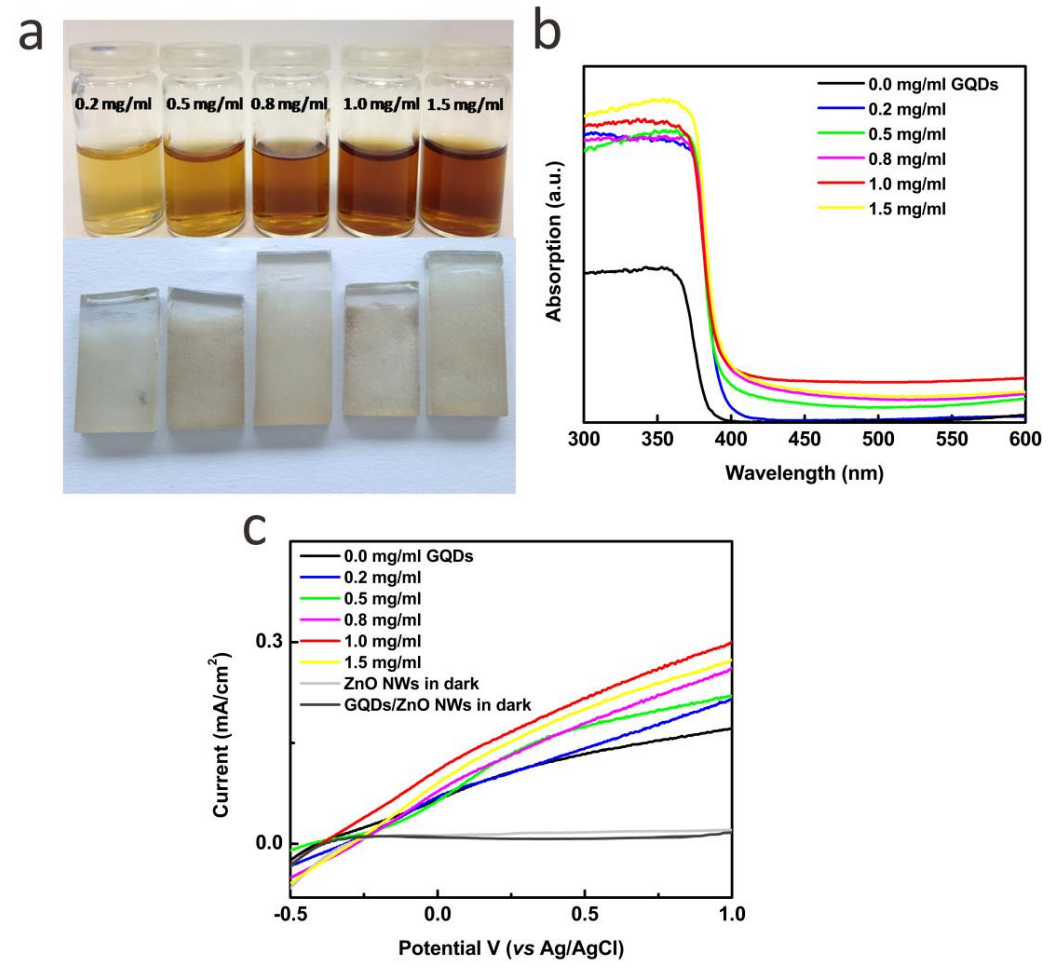

Figure 4-15. (a) Photograph of GQDs aqueous solutions with different concentrations and GQDs/ZnO NWs heterostructure fabricated with these GQDs aqueous solutions, (b) UV-vis diffuse reflectance spectra (DRS) and (c) light-responsive linear-sweep voltammograms (LSV) curves of GQDs/ZnO NWs 
heterostructures in a $0.5 \mathrm{M} \mathrm{Na}_{2} \mathrm{SO}_{4}(\mathrm{pH}=7.34)$ aqueous solution under simulated solar light irradiation $\left(100 \mathrm{mV} \mathrm{cm}^{-2}\right)$.

It is well-established that semiconductor's Fermi level in a PEC cell is equilibrated with the redox potential of electrolyte solution, which results in a band bending and generation of an electrical field.(54) Therefore, photo-induced minority charges carriers move from the semiconductor toward the interface between the semiconductor and electrolyte, by which water is either reduced to $\mathrm{H}_{2}$ in photocathode side by a p-type semiconductor or oxidized to $\mathrm{O}_{2}$ in photoanode side with an n-type semiconductor. In this regard, photoelectrochemical (PEC) water splitting reaction was utilized to evaluate the promising application of LbL-assembled N-GQDs/ZnO NWs heterostructure.

a

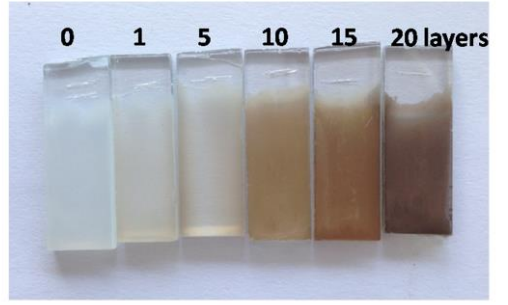

b

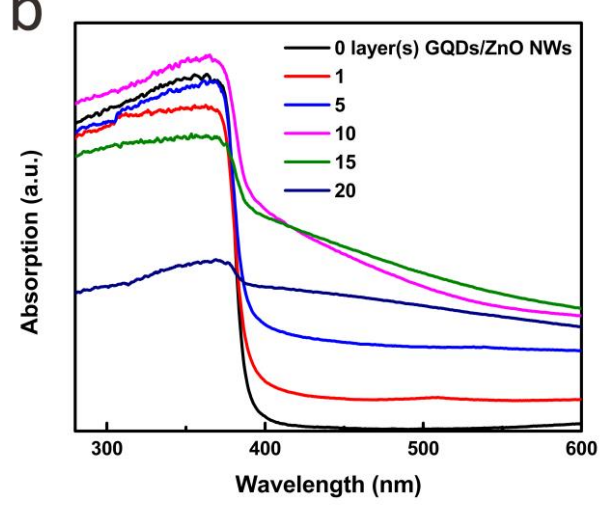

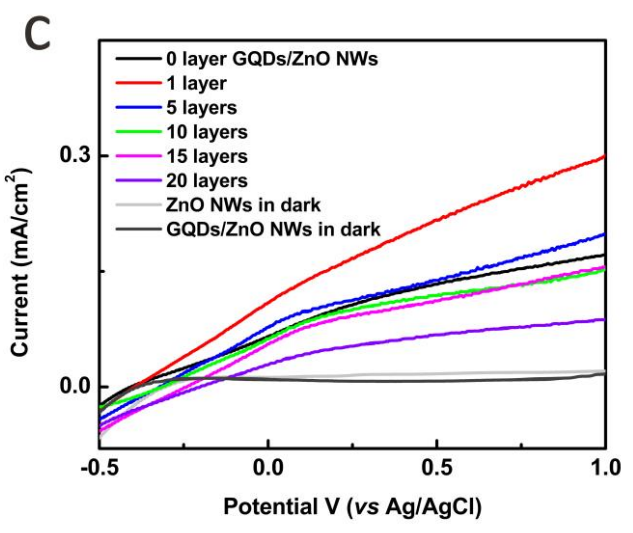

Figure 4-16 (a) Photographs, (b) UV-vis diffuse reflectance spectra (DRS), and (c) LSV curves of GQDs/ZnO NWs heterostructures with different assembly layers 
of GQDs in a $0.5 \mathrm{M} \mathrm{Na}_{2} \mathrm{SO}_{4}(\mathrm{pH}=7.34)$ aqueous solution under simulated solar light irradiation $\left(100 \mathrm{~mW} \mathrm{~cm}^{-2}\right)$.
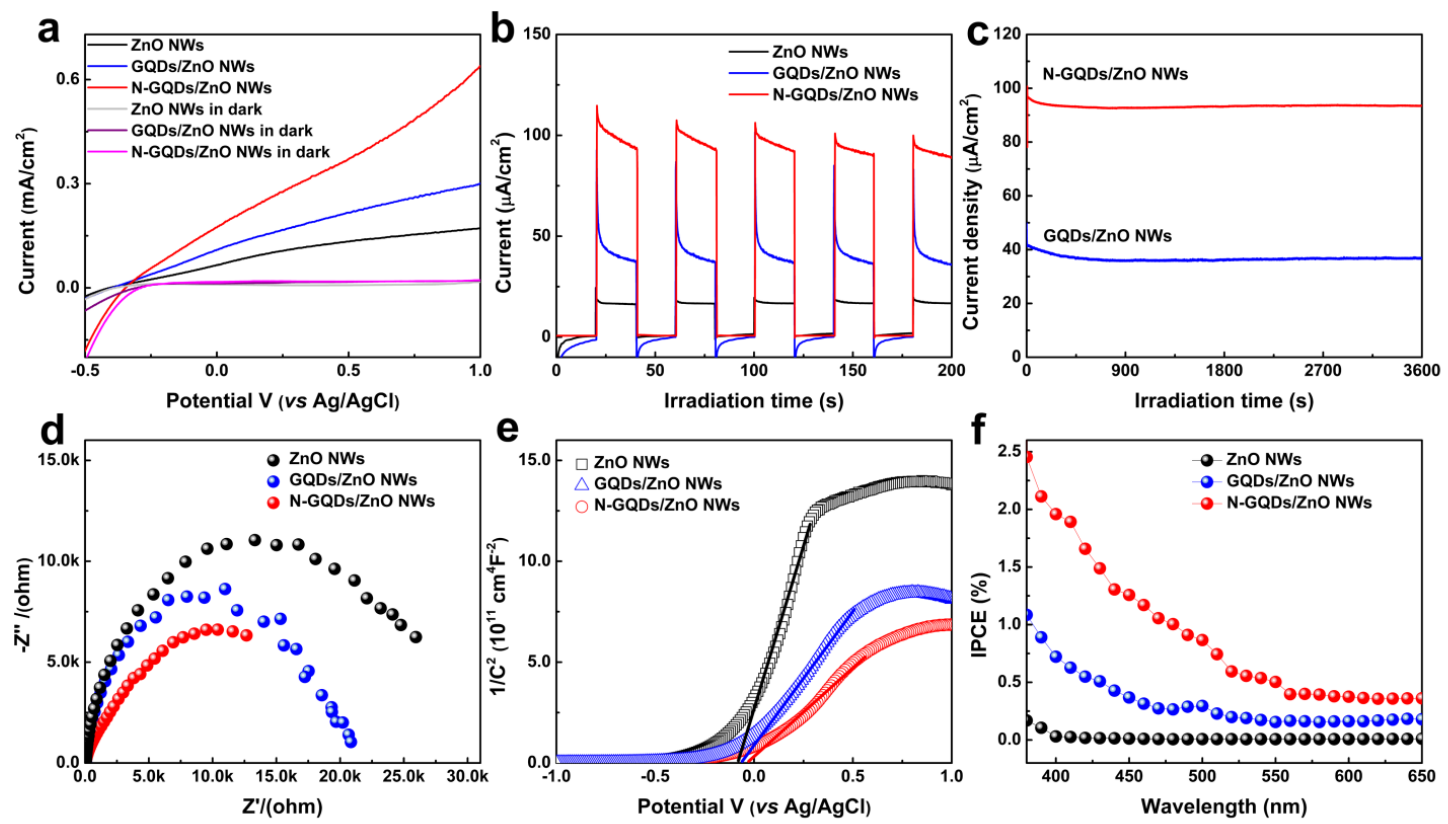

Figure 4-17 (a) Current density versus applied voltage curves, (b) transient photocurrent responses of ZnO NWs, GQDs/ZnO NWs and N-GQDs/ZnO NWs heterostructures (using $1.0 \mathrm{mg} / \mathrm{mL}$ GQDs and N-GQDs aqueous solutions with one assembly layer), and (c) photostability of GQDs/ZnO NWs and NGQDs/ZnO NWs heterostructures with zero bias vs. Ag/AgCl. (d) Electrochemical impedance spectroscopy Nyquist plots (amplitude of sinusoidal wave was set at $10 \mathrm{mV}$ and frequency varied from $100 \mathrm{KHz}$ to $0.1 \mathrm{~Hz}$ ), (e) MottSchottky curves, and (f) Incident photon-to-current conversion efficiency (IPCE) spectra with zero bias versus Ag/AgCl of different samples. The PEC measurements were carried out in a $0.5 \mathrm{M} \mathrm{Na}_{2} \mathrm{SO}_{4}$ aqueous solution $(\mathrm{pH}=7.34$ ) under simulated solar light irradiation $\left(100 \mathrm{~mW} \mathrm{~cm}^{-2}\right)$.

PEC water splitting performances of GQDs/ZnO NWs heterostructures with varied concentrations and assembly layers of GQDs were firstly systematically explored to determine the optimal experimental conditions in preparing $\mathrm{N}$ GQDs/ZnO NWs heterostructures, as displayed in Figure 4-15 and Figure 4-16. The results show that deposition amount of GQDs on $\mathrm{ZnO}$ NWs can be changed by the concentration and assembly layer of GQDs during the LbL assembly process and the optimal concentration and assembly layer of GQDs were determined to be $1.0 \mathrm{mg} / \mathrm{mL}$ 
and one assembly layer. Figure 4-17a demonstrates the photocurrent density versus applied bias voltage under simulated solar light irradiation $\left(100 \mathrm{~mW} \mathrm{~cm}^{-2}\right)$, which shows that photocurrent density of different samples increases with the applied forward potential increasing.(55) It is obvious that $\mathrm{N}$-GQDs/ZnO NWs heterostructure $(1.0 \mathrm{mg} / \mathrm{mL} \mathrm{N}-\mathrm{GQDs}$ with one assembly layer) exhibits dramatically enhanced photocurrent as compared with $\mathrm{ZnO} \mathrm{NWs}$ and GQDs/ZnO NWs counterparts within the overall voltage profile. N-GQDs/ZnO NWs heterostructures with different assembly layers were also prepared by using diluted N-GQDs aqueous solutions $(0.2 \mathrm{mg} / \mathrm{ml})$ as the building block and PEC performances of which were also probed. The results showed that N-GQDs/ZnO NWs heterostructures with five assembly layers demonstrate the optimal photocurrent as compared with other counterparts (Figure 4-18), but the value is still lower than that for the optimal sample fabricated by using $1.0 \mathrm{mg} / \mathrm{mL} \mathrm{N-GQDs.}$

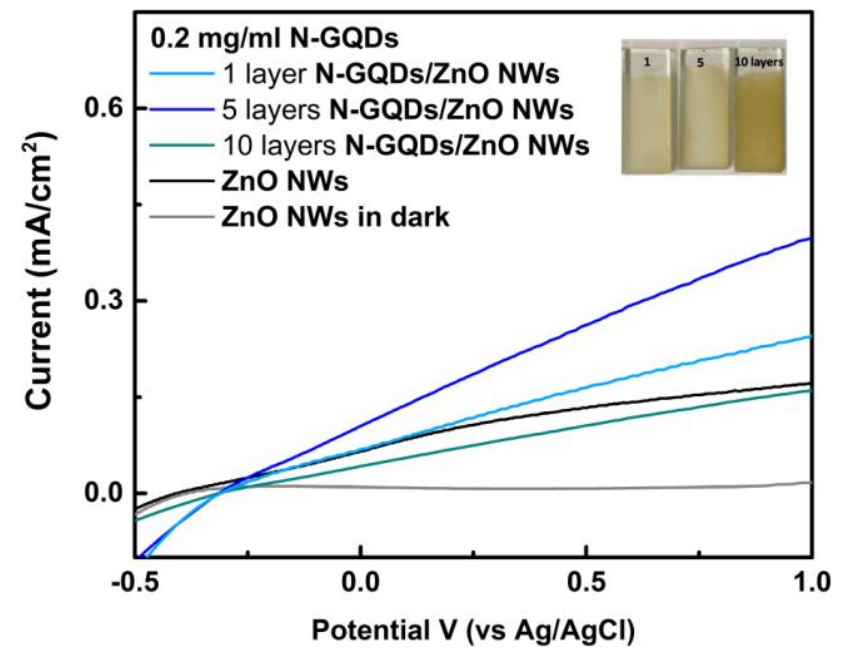

Figure 4-18 Current density versus applied voltage curves of $\mathrm{ZnO}$ NWs, and NGQDs/ZnO NWs heterostructures with different assembly layers (using 0.2 $\mathrm{mg} / \mathrm{mL} \mathrm{N}$-GQDs aqueous solutions). PEC measurements of the samples were conducted in a $0.5 \mathrm{M} \mathrm{Na}_{2} \mathrm{SO}_{4}(\mathrm{pH}=7.34)$ aqueous solution under simulated solar light irradiation $\left(100 \mathrm{~mW} \mathrm{~cm}^{-2}\right)$. 


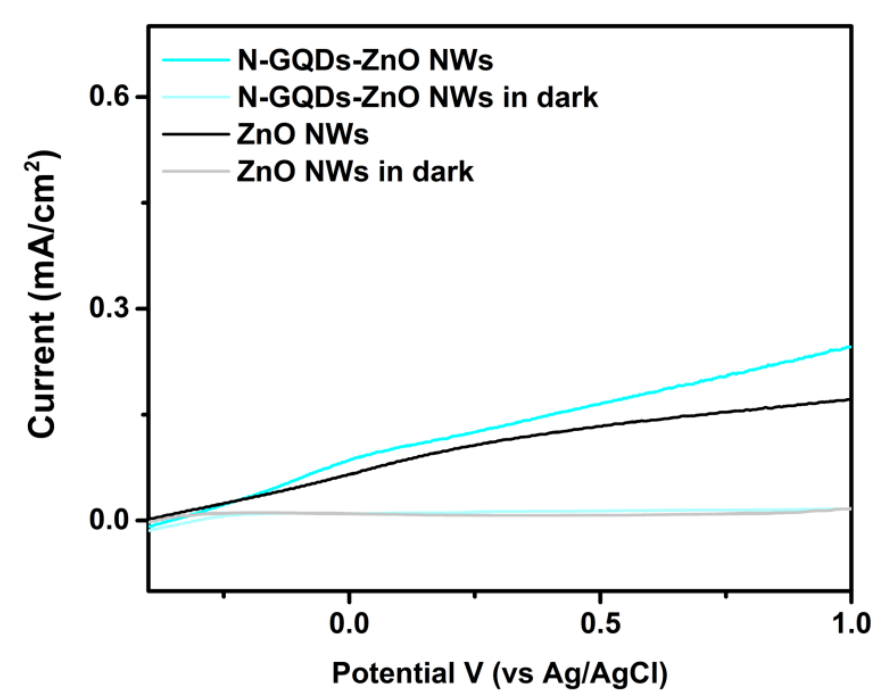

Figure 4-19 LSV curves of N-GQDs modified ZnO NWs nanocomposites prepared by a conventional coating method. PEC activities of the samples were explored in a $0.5 \mathrm{M} \mathrm{Na}_{2} \mathrm{SO}_{4}(\mathrm{pH}=7.34)$ aqueous solution under simulated solar light irradiation $\left.(100 \mathrm{~mW} \mathrm{~cm})^{-2}\right)$.

Figure 4-17b records the periodic on/off transient photocurrent response of different samples under chopped light illumination. It is evident that the photocurrent of $\mathrm{ZnO}$ NWs was remarkably improved with uniform decoration of N-GQDs. In particular, N-GQDs/ZnO NWs heterostructure demonstrates almost five-fold and two-fold photocurrent enhancement in comparison with blank $\mathrm{ZnO}$ NWs and GQDs/ZnO NWs heterostructure, respectively. The superior photocurrent of $\mathrm{N}$ GQDs/ZnO NWs heterostructures over other counterparts under the same experimental conditions suggests that photoexcited electron-hole pairs over $\mathrm{N}$ GQDs/ZnO NWs heterostructures possess the most enhanced photosensitization efficiency. It was revealed that the photo-current density of N-GQDs-ZnO NWs composite prepared by a conventional coating method is much lower than that of LbL-assembled N-GQDs/ZnO NWs heterostructure (Figure 4-19). In addition, $\mathrm{N}$ GQDs/ZnO NWs heterostructure demonstrates favorable photostability under 
continuous light irradiation and less than $3 \%$ of photocurrent decay was observed under irradiation for $1 \mathrm{~h}$ (Figure 4-17c).

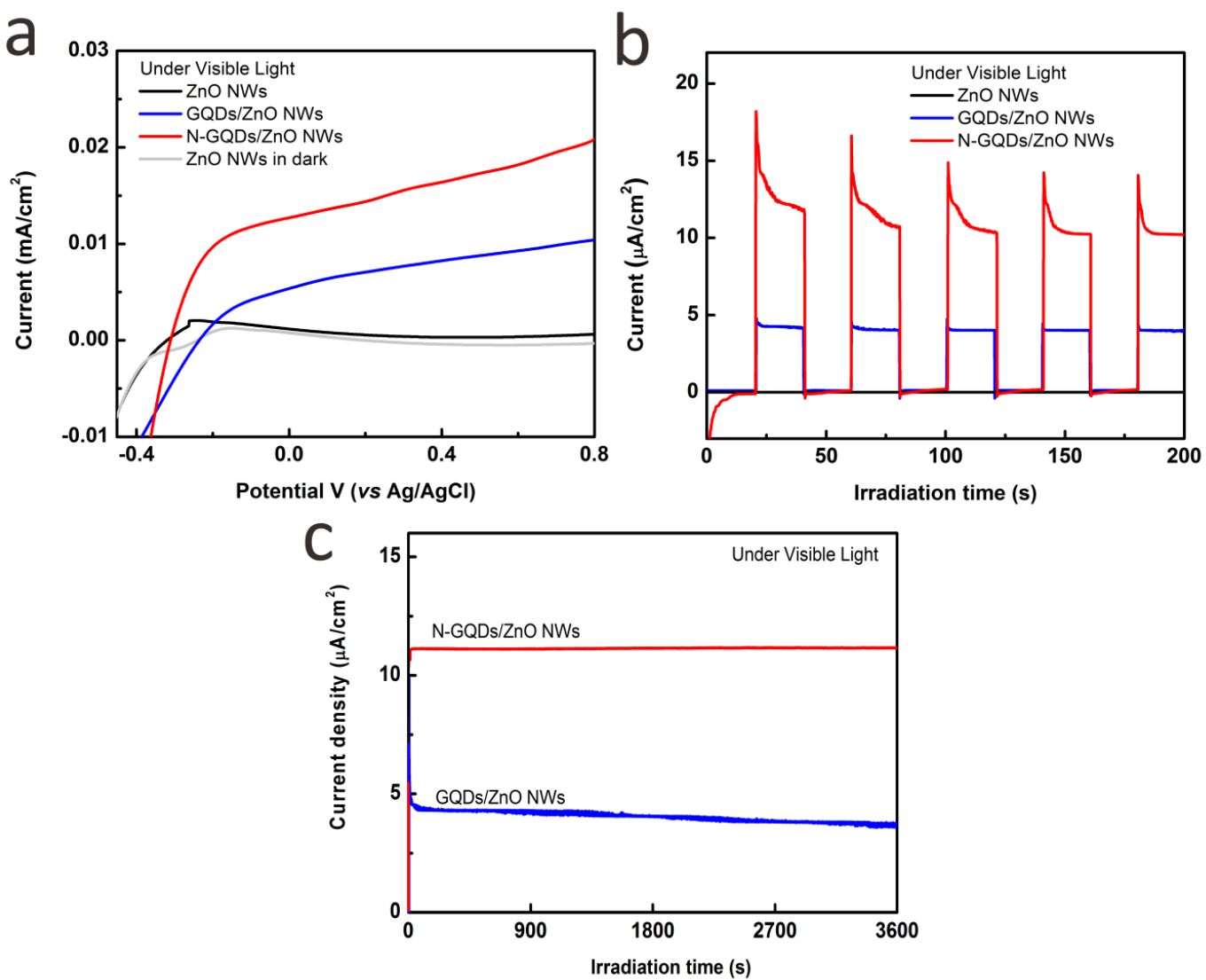

Figure 4-20. (a) Current density versus applied voltage curves, (b) transient photocurrent responses of $\mathrm{ZnO} \mathrm{NWs}$, GQDs/ZnO NWs (one assembly layer, 1.0 $\mathrm{mg} / \mathrm{mL}$ GQDs) and N-GQDs/ZnO NWs (one assembly layer, $1.0 \mathrm{mg} / \mathrm{mL} \mathrm{N}$ GQDs), and (c) photostability of GQDs/ZnO NWs and N-GQDs/ZnO NWs heterostructures (one assembly layer) in a $0.5 \mathrm{M} \mathrm{Na}_{2} \mathrm{SO}_{4}(\mathrm{pH}=7.34)$ solution with zero bias versus $\mathrm{Ag} / \mathrm{AgCl}$ under visible light $(\lambda>420 \mathrm{~nm})$ illumination.

On a separate set of experiments when visible light $(\lambda>420 \mathrm{~nm})$ illumination was used (Figure 4-20), a similar photocurrent enhancement of $\mathrm{N}$-GQDs/ZnO NWs heterostructure was also observed. LSV results of different samples under visible light irradiation indicate that $\mathrm{N}-\mathrm{GQDs} / \mathrm{ZnO}$ heterostructure exhibits the most superior photocurrent as compared with $\mathrm{ZnO}$ NWs and GQDs/ZnO NWs counterparts (Figure 4-20a). Consistently, periodic on/off transient photocurrent responses of different 
samples under intermittent visible light irradiation in Figure 4-20b demonstrate the same trend. Alternatively, as shown in Figure 4-20c, N-GQDs/ZnO NWs and GQDs/ZnO NWs heterostructures demonstrate favorable photostability under continuous visible light irradiation for $1 \mathrm{~h}$, which is analogous to photostability of composite nanostructures under simulated solar light irradiation.

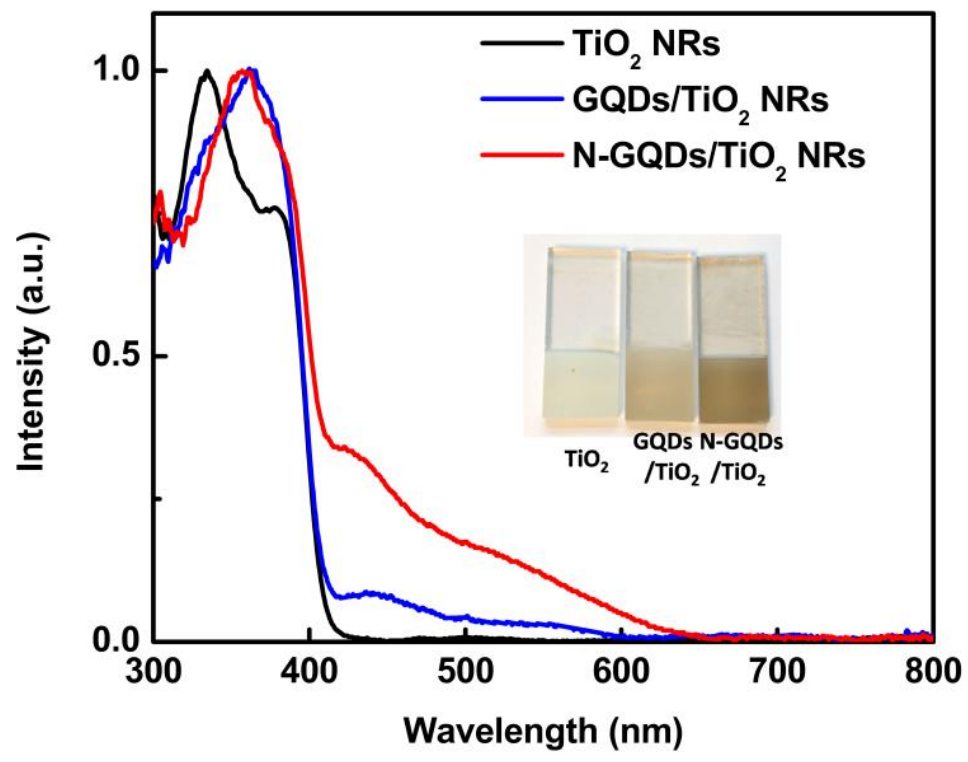

Figure 4-21. UV-vis diffuse reflectance spectra (DRS) of $\mathrm{TiO}_{2} \mathrm{NRs}, \mathrm{GQDs}_{\mathbf{T}} \mathrm{TiO}_{2}$ NRs (one assembly layer) and $\mathrm{N}-\mathrm{GQDs} / \mathrm{TiO}_{2}$ NRs heterostructure (one assembly layer).

Analogous to DRS results of $\mathrm{ZnO}$ NWs based composites, $\mathrm{N}-\mathrm{GQDs} / \mathrm{TiO}_{2} \mathrm{NRs}$ and GQDs/ $/ \mathrm{TiO}_{2}$ NRs heterostructures exhibit pronounced absorption in visible region, while no absorption in the same wavelength region was observed for $\mathrm{TiO}_{2} \mathrm{NRs}$, indicating the pivotal role of N-GQDs and GQDs as photosensitizers. Moreover, it is worthwhile that $\mathrm{N}-\mathrm{GQDs} / \mathrm{TiO}_{2}$ NRs heterostructures demonstrates significantly enhanced light absorption in visible region as compared with GQDs/TiO 2 NRs counterpart with the same assembly layer, which highlights the contributing role of nitrogen doping in boosting light absorption of GQDs and GQDs/TiO 2 NRs heterostructure. More significantly and consistently, N-GQDs/TiO ${ }_{2}$ nanorods (NRs) 
heterostructure (one assembly layer) exhibit more significant photocurrent enhancement than GQDs/ $\mathrm{TiO}_{2} \mathrm{NRs}$ and $\mathrm{TiO}_{2} \mathrm{NRs}$ under the same experimental conditions, following the order of: $\mathrm{N}-\mathrm{GQDs} / \mathrm{TiO}_{2} \mathrm{NRs}>\mathrm{GQDs} / \mathrm{TiO}_{2} \mathrm{NRs}>\mathrm{TiO}_{2}$ NRs (Figure 4-21 and 4-22). Consequently, based on the above analysis, an extraordinary photosensitization effect of N-GQDs on the PEC water splitting performances of LbL-assembled N-GQDs/1D semiconductor ( $\mathrm{ZnO} \mathrm{NWs}$ or $\mathrm{TiO}_{2}$ NRs) heterostructures was unambiguously ascertained under both visible light and simulated solar light illumination.
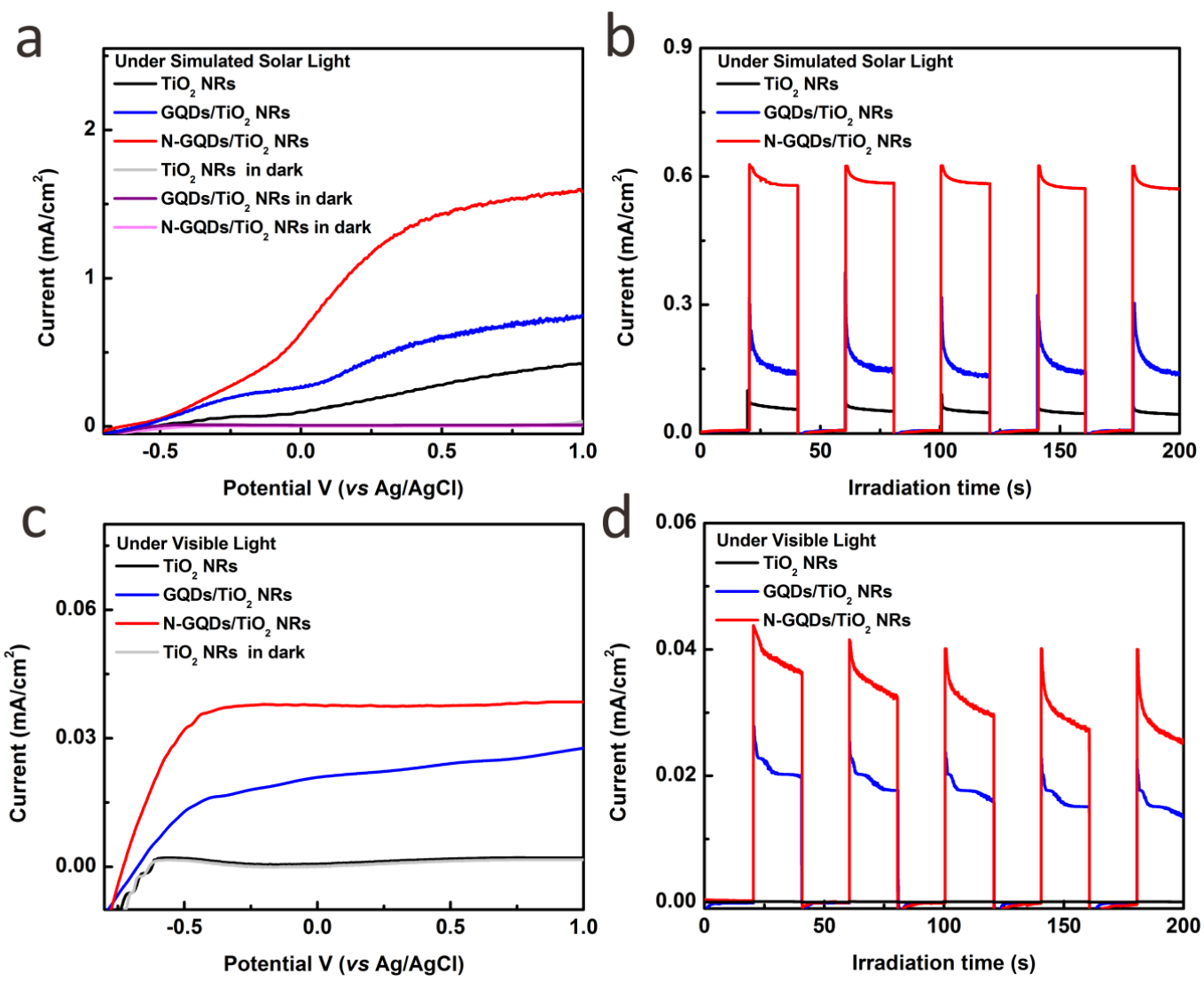

Figure 4-22 (a, c) Current density versus applied voltage curves, and (b, d) transient photocurrent responses of $\mathrm{TiO}_{2} \mathrm{NRs}, \mathrm{GQDs} / \mathrm{TiO}_{2} \mathrm{NRs}$ and $\mathrm{N}$ GQDs/TiO 2 NRs heterostructures (using $1.0 \mathrm{mg} / \mathrm{mL}$ GQDs and N-GQDs aqueous solutions with one assembly layer). The PEC measurements were carried out in a $0.5 \mathrm{M} \mathrm{Na}_{2} \mathrm{SO}_{4}$ aqueous solution $(\mathrm{pH}=7.34)$ under simulated solar or visible light irradiation $\left(100 \mathrm{~mW} \mathrm{~cm}^{-2}\right)$. 
To probe the separation efficiency of photogenerated charge carriers over $\mathrm{N}$ GQDs/ZnO NWs heterostructure, electrochemical impedance spectroscopy (EIS) measurements of different samples under light illustration were performed (Figure 417d).(56,57) The Nyquist plot from that of $\mathrm{N}-\mathrm{GQDs} / \mathrm{ZnO}$ NWs heterostructure exhibits the smallest semicircle radius than that of blank $\mathrm{ZnO}$ NWs and GQDs/ZnO NWs under light irradiation, indicating charge transfer resistance in the interface of $\mathrm{ZnO}$ NWs and electrolyte was reduced in the presence of N-GQDs, highlighting the key benefit of nitrogen doping in boosting charge transfer and hence the photocurrent generated. Mott-Schottky (M-S) plots are shown in Figure 4-17e, in which slopes of the linear parts of all M-S plots are not negative, indicative of n-type semiconductor property.(206) In the M-S plots, the linear parts can be further extrapolated to $1 / C^{2} \rightarrow$ 0 to estimate the Fermi level $\left(\mathrm{U}_{\mathrm{FL}}\right)$ values of $\mathrm{ZnO} \mathrm{NWs}$, GQDs/ZnO NWs and $\mathrm{N}$ GQDs/ZnO NWs heterostructures as $-0.0855,-0.0581$ and $-0.0212 \mathrm{~V}$ (vs $\mathrm{Ag} / \mathrm{AgCl}$ ), respectively. The pronounced positive shift of $\mathrm{U}_{\mathrm{FL}}$ for $\mathrm{N}-\mathrm{GQD} / \mathrm{ZnO} \mathrm{NWs}$ in comparison with $\mathrm{ZnO} \mathrm{NWs}(36.9 \mathrm{mV}$ vs $\mathrm{Ag} / \mathrm{AgCl})$ and GQDs/ZnO NWs (27.4 mV vs $\mathrm{Ag} / \mathrm{AgCl}$ ) suggests that modification of $\mathrm{ZnO} \mathrm{NWs}$ with $\mathrm{N}$-GQDs leads to a decrease in band bending edge, which is conducive to electron transfer from N-GQDs to $\mathrm{ZnO}$ under light irradiation. Consequently, $\mathrm{M}-\mathrm{S}$ result indicates that $\mathrm{N}-\mathrm{GQD} / \mathrm{ZnO}$ NWs heterostructure demonstrates the most enhanced charge transfer efficiency when compared with $\mathrm{ZnO} \mathrm{NWs}$ and GQDs/ZnO NWs counterparts, thus resulting in the significantly improved PEC water splitting performances. The carrier density $\left(\mathrm{N}_{\mathrm{D}}\right)$ of different samples can be estimated by the following equation:(206)

$$
N_{\mathrm{D}}=\left(2 / e \varepsilon_{0} \varepsilon_{r}\right)\left[d U_{F L} / d\left(1 / C^{2}\right)\right]
$$

where e $=1.6 \times 10^{-19} \mathrm{C}, \varepsilon_{0}=8.86 \times 10^{-12} \mathrm{~F} / \mathrm{m}, \varepsilon_{r}=8.12$ for wurtzite $\mathrm{ZnO},{ }^{[58]}$ and C represents the capacitance. The $N_{D}$ results of $\mathrm{ZnO}$, GQDs/ZnO NWs and $\mathrm{N}$ - 
GQDs/ZnO NWs heterostructure were determined to be $4.5 \times 10^{18}, 1.02 \times 10^{19}$ and $1.61 \times 10^{19} \mathrm{~cm}^{-3}$, respectively. The larger $N_{D}$ value of $\mathrm{N}-$ GQDs/ZnO NWs heterostructure suggests more efficient charge transfer efficiency as compared with $\mathrm{ZnO}$ and GQDs/ZnO NWs counterparts and, thus, more significantly enhanced PEC water splitting performances.

Incident photon-to-current conversion efficiency (IPCE) spectra of different samples were further collected under monochromatic light irradiation versus incident light wavelength according to the following equation:(59)

$$
\mathrm{IPCE}=(1240 \mathrm{I}) /\left(\lambda \mathrm{J}_{\text {light }}\right)
$$

where $I$ is the photocurrent density, $\lambda$ is the incident light wavelength, and $J_{\text {light }}$ is the incident light intensity. As shown in Figure 4-17f, N-GQDs/ZnO NWs heterostructure exhibits noticeable photoresponse in visible region up to $600 \mathrm{~nm}$, while $\mathrm{ZnO}$ NWs exhibit no photoresponse above $400 \mathrm{~nm}$, which are in faithful agreement with DRS results. Compared with $\mathrm{ZnO}$ NWs based PEC cell, NGQDs/ZnO NWs photoelectrode reveals an IPCE of $c a .0 .86 \%$ at a wavelength of $500 \mathrm{~nm}$ with zero applied voltage (vs $\mathrm{Ag} / \mathrm{AgCl})$, which is $c a .50$ times and 3.0 times larger than those of blank ZnO NWs $(0.017 \%)$ and GQDs/ZnO NWs (0.29 \%), respectively. Due to the negligible absorption at $500 \mathrm{~nm}$ for $\mathrm{ZnO}$ NWs, the clearly resolved peak in IPCE spectrum of N-GQDs/ZnO NWs heterostructure can only be attributed to N-GQDs. Accordingly, IPCE results confirm that N-GQDs acts as a highly efficient photosensitizer and remarkably promotes the light absorption of $\mathrm{ZnO}$ NWs in visible region, thereby giving rise to significantly enhanced PEC watersplitting performances of N-GQDs/ZnO NWs heterostructure. 

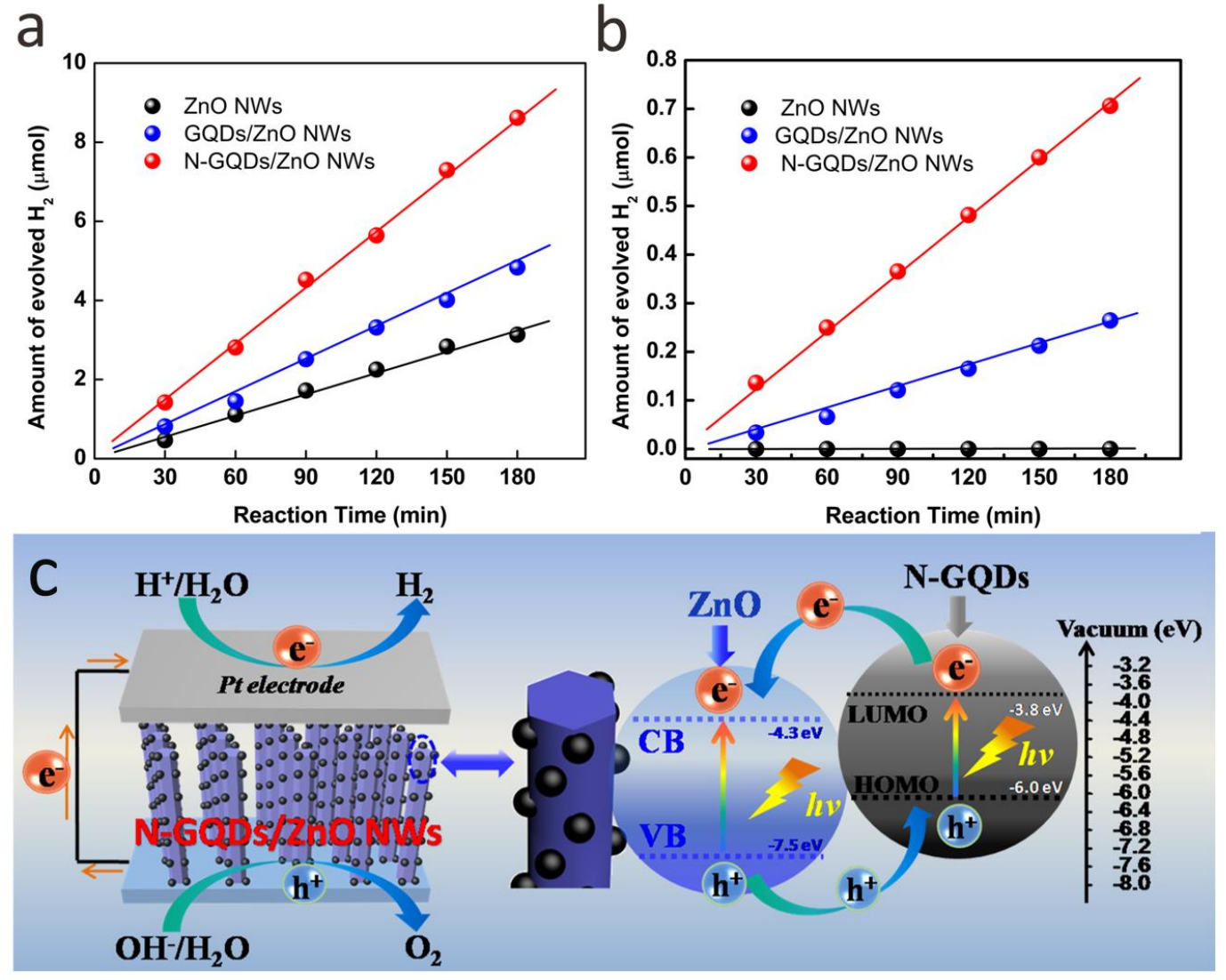

Figure 4-23. Hydrogen evolution performances of $\mathrm{ZnO} \mathrm{NWs}$, GQDs/ZnO NWs and M-GQDs/ZnO NWs in $0.5 \mathrm{M} \mathrm{Na}_{2} \mathrm{SO}_{4}$ aqueous solution, (a) under simulated solar light illumination (100 $\left.\mathrm{mW} \mathrm{cm}^{-2}\right)$, and (b) under visible light illumination (> $420 \mathrm{~nm}$ ). (c) Schematic illustration of PEC water splitting mechanism over NGQDs/ZnO NWs heterostructure under light irradiation.

To further evaluate the PEC performances of the different samples for hydrogen production, a gas-enclosed system with a gas chromatography under the same simulated solar or visible light irradiation was used to detect the amount of evolved gas. The amount of $\mathrm{H}_{2}$ evolved over $\mathrm{ZnO} \mathrm{NWs}$, GQDs/ZnO NWs, and N-GQDs/ZnO NWs under simulated solar light illumination for $180 \mathrm{~min}$ is shown in Figure 4-23a, which indicates that the amount of $\mathrm{H}_{2}$ generated almost increased linearly with the irradiation time, and the $\mathrm{H}_{2}$ amount produced over $\mathrm{N}-\mathrm{GQD} / \mathrm{ZnO} \mathrm{NWs}$ heterostructure is nearly 4 and 2 times higher than those produced over blank $\mathrm{ZnO}$ NWs and GQDs/ZnO NWs, respectively, indicative of a synergistic contribution from 
nitrogen doping and photosensitization effect of GQDs in enhancing PEC hydrogen production performances of N-GQDs/ZnO NWs. Analogous results were observed under visible light irradiation over different samples, from which N-GQDs/ZnO NWs exhibits the largest hydrogen production amount in comparison with $\mathrm{ZnO}$ NWs and GQDs/ZnO NWs, as displayed in Figure 4-23b. Therefore, consistent with PEC results which evidenced strong photocurrent enhancement, the current study strongly suggests that $\mathrm{N}-\mathrm{GQDs} / \mathrm{ZnO} \mathrm{NWs}$ heterostructure can serve as an efficient electrode for hydrogen production under both visible light and simulated solar light illumination.
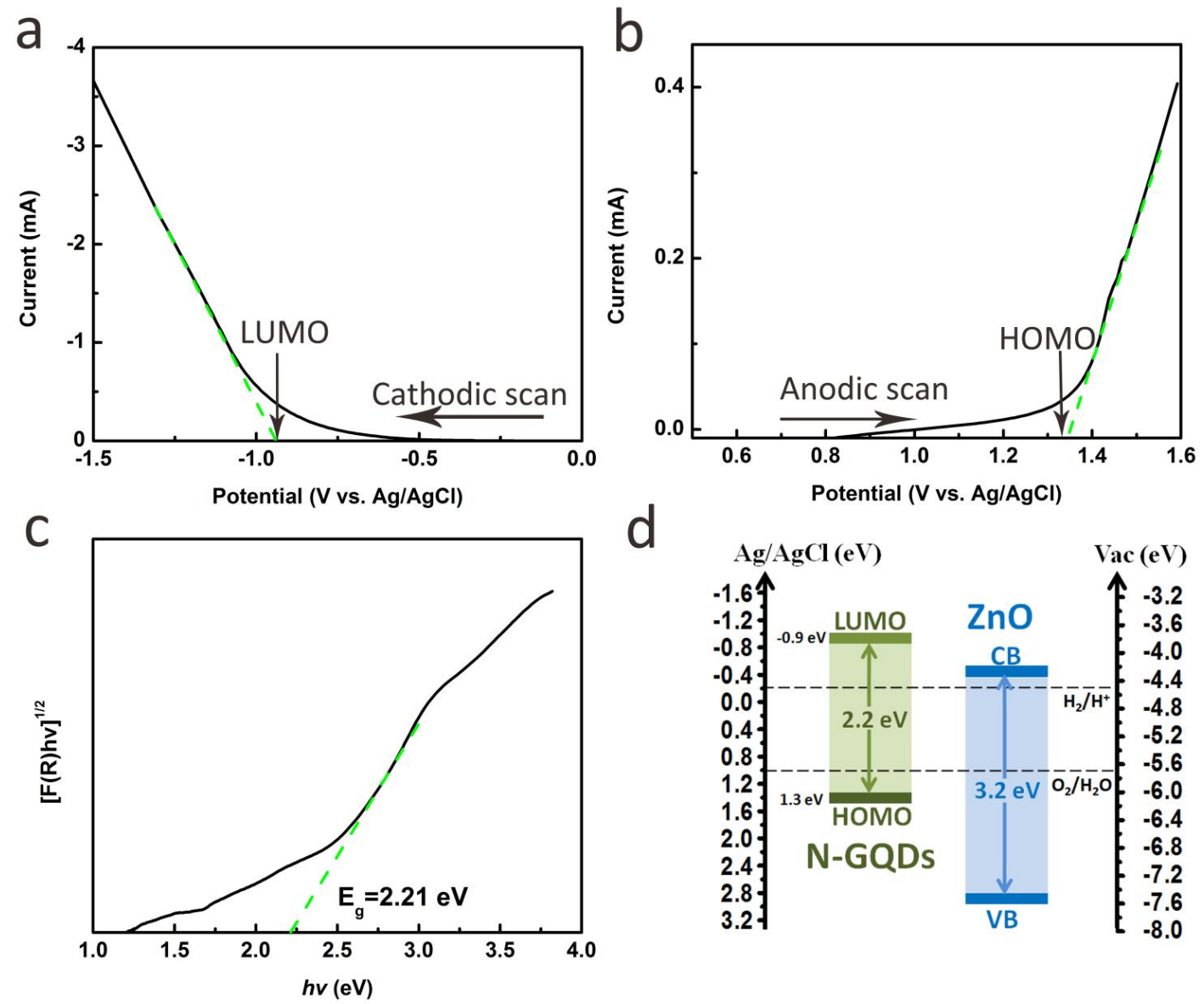

Figure 4-24 (a) Cathodic scan to indentify the LUMO energy level of N-GQDs (scan rate: $5 \mathrm{mV} / \mathrm{s}$ ), (b) anodic scan to indentify the HOMO energy level of $\mathrm{N}$ GQDs (scan rate: $5 \mathrm{mV} / \mathrm{s}$ ). (c) Plots of transformed Kubelka-Munk function versus the energy of light for N-GQDs. (d) Schematic illustration of energy levels of N-GQDs and ZnO NWs. 
The significant enhancement of hydrogen generation using the current $\mathrm{N}$ GQDs/ZnO NWs heterostructure by proposing a PEC water splitting mechanism was illustrated in Figure 4-23c. Based on Kohn-Sham molecular orbitals (MOs) theory, a large variety of electron transitions in N-GQDs can be triggered from the occupied orbitals (i.e., Highest occupied molecular orbital, HOMO) to the unoccupied orbitals (i.e., Lowest unoccupied molecular orbital, LUMO) under light irradiation, therefore endowing N-GQDs with a favorable HOMO-LUMO gap (Figure 4-24) which performs as a semiconductor with a relative small bandgap.(16,60) In this way, NGQDs in our reaction system can be photoexcited under simulated solar or visible light irradiation, generating electron-hole pairs due to the favorable bandgap of $\mathrm{N}$ GQDs/ZnO NWs heterostructure (2.85 eV, Figure 4-12). In addition, considering the LUMO potential of N-GQDs (Figure 4-24d) is more negative than the conduction band $(\mathrm{CB})$ edge of $\mathrm{ZnO},(16,60,61)$ and an intimate N-GQDs-to-ZnO NWs interfacial interaction resulted from the LbL strategy, photoexcited electrons produced in-situ from N-GQDs under light irradiation can be spontaneously transferred to the CB of ZnO. Thus, photogenerated electrons and holes over N-GQDs are effectively separated and the lifetime of photogenerated charge carriers is remarkably prolonged. Simultaneously, it should be noted that $\mathrm{ZnO}$ NWs substrate can also be photoinduced under simulated solar light illumination in which electrons are excited from the valence band (VB) to the CB leaving holes in the VB. Under simulated solar light irradiation, both $\mathrm{ZnO}$ and N-GQDs were photoexcited giving rise to photogenerated charge carriers. The photogenerated holes on the valence band (VB) of $\mathrm{ZnO}$ can transfer to the HOMO of N-GQDs owing to their favorable band alignment and then oxide water to oxygen on the working electrode. Simultaneously, photoelectrons from LUMO of N-GQDs transfer to the $\mathrm{CB}$ of $\mathrm{ZnO} \mathrm{NWs}$ and then to the external circuit 
producing photocurrent and ultimately reduce water to hydrogen on the counter electrode. When $\mathrm{N}-\mathrm{GQDs} / \mathrm{ZnO} \mathrm{NWs}$ heterostructure was irradiated under visible light irradiation $(\lambda>420 \mathrm{~nm})$, only N-GQDs can be photoexcited producing photoelectrons on the LUMO (Figure 4-25). Subsequently, the electrons transfer to the $\mathrm{CB}$ of $\mathrm{ZnO}$ and finally reduced water to hydrogen and the hole on the LUMO oxidize water to oxygen.

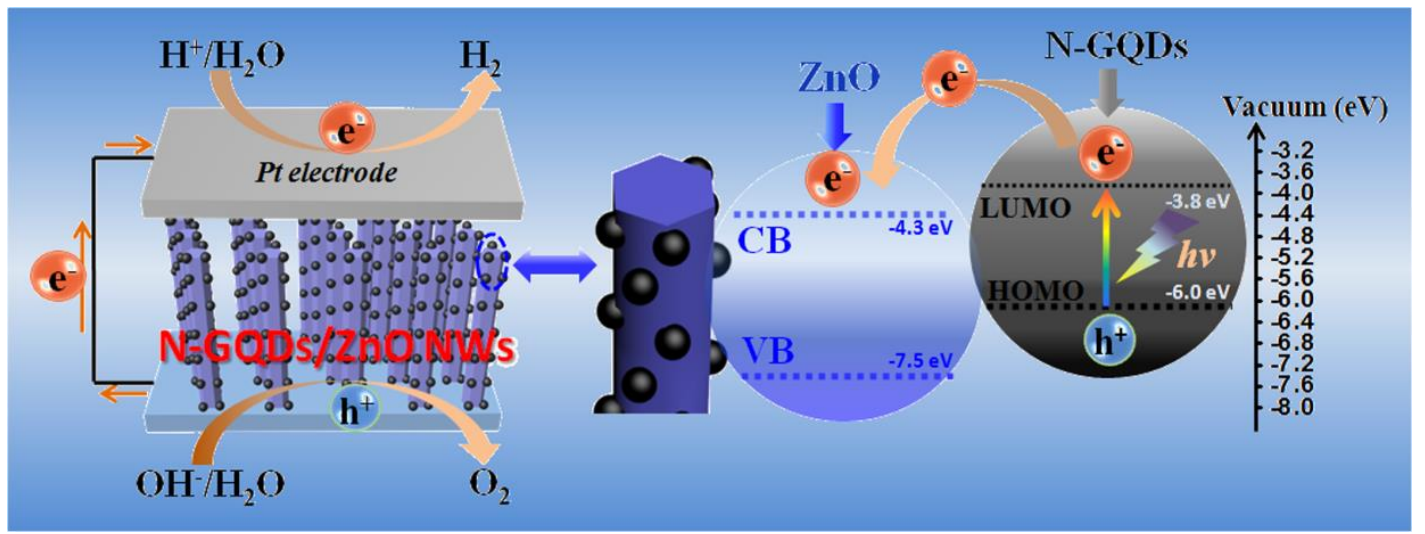

Figure 4-25 Schematic illustration of PEC water splitting mechanism over NGQDs/ZnO NWs heterostructure under visible light irradiation.

\subsection{Summary}

In summary, highly ordered N-GQDs decorated 1D semiconductor (ZnO NWs or $\mathrm{TiO}_{2} \mathrm{NRs}$ ) nanohybrid heterostructures have been fabricated by a simple LbL assembly strategy based on strong electrostatic interaction. We can demonstrate that the well-defined LbL-assembled N-GQDs/ZnO NWs (as well as its N-GQDs/TiO 2 NRs counterpart) photoanode resulted in significantly enhanced PEC water splitting performances under both simulated solar and visible light irradiations. The enhanced PEC performances are attributed to the extraordinary photosensitization effect of $\mathrm{N}$ GQDs and intimate interfacial interaction between $\mathrm{N}$-GQDs and $\mathrm{ZnO} \mathrm{NWs}$ framework afforded by nitrogen doping and LbL assembly. More significantly, PEC water splitting performances of N-GQDs/1D semiconductor heterostructures can be 
tuned by assembly layer. It is anticipated that our work could provide a general synthetic approach to prepare a large diversity of N-GQDs/semiconductor nanoarchitectures and afford new insights for the potential applications of N-GQDs in the field of photocatalysis, photoelectrocatalysis and photoelectrochemical systems. 


\section{Chapter 5 Graphene Oxide Quantum Dots}

\section{Covalently Functionalized PVDF Membrane with}

\section{Significantly-Enhanced Bactericidal and}

\section{Antibiofouling Performances ${ }^{2}$}

\subsection{Introduction}

One of the most pervasive challenges afflicting the human race is inadequate access to clean water and sanitation,(87-89) and this due to rapid technological advancement going in tandem with high population growth. A huge threat to public health worldwide is caused by pathogenic microorganisms in natural and sewage water. Membrane technologies are finding wide applications in water treatment,(90) but the growth of pathogenic microorganisms on the membrane surface can produce a fouling layer, i.e., biofilm, and this leads to significant decrease in water flux and increase in energy consumption. Functionalization of water filtration membranes with microorganism resistance property is therefore important in overcoming pathogenic contamination and biofouling.(91,92) Poly(vinylidene fluoride) (PVDF) microfiltration and ultrafiltration membranes, widely used in industrial wastewater and municipal water treatment, possess excellent chemical and thermal stability, high resistance to radiation and strong mechanical property.(93) However, PVDF is intrinsically hydrophobic and therefore easily contaminated by microorganism, resulting in serious membrane fouling.(94) Therefore, new modification strategies of

\footnotetext{
${ }^{2}$ Reproduced from [Zeng Z., Yu D., He Z., Liu J., Xiao F. -X., Zhang Y., Wang R., Bhattacharyya D., Tan T. Y. T., Scientific Report, 2016, 6, 20142.] with permission from Nature Publish Group
} 
PVDF membrane for effective and long-lasting antibacterial and antibiofouling activities will be highly attractive.

Incorporating biocidal nanostructure materials into the membrane has been regarded as an efficient way to impart antimicrobial and antibiofouling properties to the membrane. Biocide-releasing nanomaterials, such as nano-metal oxide (zinc oxide),(220) silver and copper nanoparticles, $(221,222)$ have been introduced inside the membrane. However, these nanomaterials are often leached out during the water purification process, resulting in secondary contamination.(92) Alternatively, carbonbased nanomaterials, including one-dimensional $(1 \mathrm{D})$ carbon nanotubes and twodimensional (2 D) graphene nanosheets, have been explored as excellent antimicrobial reagents due to their cytotoxic mechanisms of physical piercing and oxidative stress.(91,223-226) For example, biocidal single-walled carbon nanotubes (SWNTs) and graphene oxide (GO) nanosheets can be used to functionalize polyamide membranes by Elimelech's group,(224,226) showing enhanced antibacterial activity. However, these $1 \mathrm{D}$ or $2 \mathrm{D}$ carbon materials on membranes still cannot be highly efficient in inhibiting the colonization of bacteria.

Graphene oxide quantum dots (GOQDs) with the diameters in the range of 3-20 $\mathrm{nm},(227)$ have attracted extensive attention in the antibacterial research area due to their unique properties. Compared with bulk graphene oxide (GO) nanosheets, GOQDs exhibit zero-dimensional (0 D) distinctive optical and electronic properties due to their large edge effects and quantum confinement. This results in better peroxidase-like activity than graphene nanosheets because of their unique electron transport property.(64,190,228,229) More importantly, previous studies have shown that $0 \mathrm{D}$ GOQDs also exhibit lower cytotoxicity than that of the micrometer-sized 
graphene oxide sheets in a long-term in vivo study. $(230,231)$ However, to the best of our knowledge, GOQDs have rarely been explored as bactericidal reagents in membranes. The fabrication of antimicrobial, mechanically strong and highpermeability membranes using $2 \mathrm{D}$ graphene sheets incorporated into polymer membrane through mixed-solution casting method has been reported.(232,233) However, the solution-casting method may not be preferable, since it leads to the embedment of most graphene materials, inside the membrane, and only a small fraction of graphene is exposed on the surface to exert their functions.(226) In order to fully utilize the surface-anchored graphene so as to enable the direct contact with bacteria and significantly reduce the amounts of nanomaterials required, it is highly desirable to homogenously immobilize graphene-based materials on the membrane surface.

In this work, we report a new type of GOQDs covalent-surface functionalized PVDF membrane possessing attractive combined features of hydrophilicity, stability, antibiofouling, and antibacterial properties. Structural analysis, hydrophilicity, water permeability, antibiofouling, and antibacterial performance of the membranes were assessed for its preliminary suitability in water purification processes. It was found that the GOQDs anchored PVDF membrane demonstrated significantly-improved antibacterial and antibiofouling performance, while retaining the permeating properties of the pristine PVDF membrane. Its antibacterial activity is also superior to previously reported $2 \mathrm{D}$ GO sheets and $1 \mathrm{D}$ carbon nanotube modified polymer membranes.(223-226) 


\subsection{Experimental section}

\subsubsection{Fabrication of graphene nanosheets (GO), GO-PVDF and GQDs-PVDF Synthesis of graphene oxide quantum dots (GOQDs) and graphene oxide nanosheets (GO)}

Graphene oxide quantum dots (GOQDs) were synthesized with CX-72 carbon black via being refluxed in concentrated nitric acid $\left(\mathrm{HNO}_{3}\right)$ solution.(211) Typically, $0.4 \mathrm{~g}$ dried CX-72 carbon black was added to $6 \mathrm{~mol} \mathrm{~L}^{-1}$ nitric acid $(100 \mathrm{ml})$ and refluxed for one day at $110{ }^{\circ} \mathrm{C}$. After cooling to below $30{ }^{\circ} \mathrm{C}$, the product was centrifuged (12000 rpm) for $10 \mathrm{~min}$ to achieve sediment and a supernatant. The resulting supernatant was kept at $200{ }^{\circ} \mathrm{C}$ to evaporate the nitric acid and water. After cooling to the room temperature, a reddish-brown solid was acquired. Then, GOQDs solution can be obtained by dissolving the GOQDs in DI water under $10 \mathrm{~min}$ sonication. Graphene oxide nanosheets (GO, Figure S2) were fabricated via the modified hummers' method.(234)

\section{Fabrication of GO-PVDF/GOQDs-PVDF}

PEG (15 g) was put into DI water (300 g) to prepare a PEG solution (5 wt \%). PVDF membrane (pore size $0.22 \mu \mathrm{m}$, GVHP from Millipore) was immersed in the PEG solution for 30 min, subsequently dried in the air. The pretreated membrane can be dropped between the biased electrode and ground electrode in a plasma chamber. He gas was then channeled into the chamber when the pressure of the reactor system was 0-100 $\mathrm{Pa}$. The radio frequency $(\mathrm{RF})$ power of discharging gas plasma was $100 \mathrm{~W}$, while the exposure time was 120 s. To remove the residual PEG, the resultant membrane was then washed by DI water. The resulting membrane was dried at $60{ }^{\circ} \mathrm{C}$ by using a vacuum oven for characterization and preparation. 
The resulting membrane was dipped into $0.2 \mathrm{wt} \%$ (3-aminopropy) trimethoxysilane (APTMS) in iso-propanol (IPA) for $30 \mathrm{~min}$. The membrane was subsequently rinsed and immersed into DI water for $10 \mathrm{~min}$, and the washing process was repeated for 3 times. After that, the membrane was dried under nitrogen flow. The obtained membrane was then dipped into an aqueous solution of GO, or GOQDs (1 $\mathrm{mg} / \mathrm{ml}$ ) with ethyl(dimethylaminopropyl) carbodiimide/n-hydroxysuccinimide (EDC/NHS) for $3 \mathrm{~h}$. Then, the membrane was again rinsed and immersed by DI water to remove the residual GO. The resulting GO-PVDF or GOQDs-PVDF was dried with nitrogen flow.

\subsubsection{Materials characterization}

Scanning electron microscopy (SEM) images can be acquired with field emission scanning electron microscopy (JEOL, JSM-6700F). Transmission electron microscopy (TEM) images were achieved by using a JEOL model JEM2010 (200 $\mathrm{kV}$ ). UV-vis spectra were recorded by using a Shimadzu UV2501 spectrophotometer. The fluorescence properties were studied using a Fluoromax-4, Horiba Jobin Yvon Spectrofluorometer with a photon-counting detection system to detect fluorescence emission. Fourier Transform Infrared Spectroscopy (FTIR) was collected by using a Digilab FTS 3100 instrument. Membrane surface charges were measured through using streaming potential method with a SurPASS electrokinetic analyzer (Anton Paar GmbH, Austria).(235,236)

\subsubsection{Antimicrobial activities assay}

The antimicrobial activity of the composite films was evaluated by a plate counting method. Before counting a functionalized filter for antimicrobial activities, the presence of graphene on the filter was confirmed by performing water contact 
angle measurements for each filter. Both E. coli and S. aureus bacteria were prepared in Luria-Bertani (LB) medium at $37{ }^{\circ} \mathrm{C}$ for overnight with shaking, and then washed three times with fresh sterile $0.9 \%$ saline. The bacteria solution was diluted to $\sim 10^{7}$ colony-forming units per milliliter $\left(\mathrm{CFU} \mathrm{mL} \mathrm{mL}^{-1}\right)$ by using sterile $0.9 \%$ saline solution. All the solutions and samples (except the composite films) in the experiment need to be autoclaved at $120{ }^{\circ} \mathrm{C}$ for about 20 min to ensure sterility. Antibacterial activities were explored with the antibacterial drop-test method.(226) For composite film exposure, pristine PVDF and composite films with an area of $2 \mathrm{~cm}^{2}$ are placed in a plastic holder to expose the active surface to the bacteria. Bacterial suspensions $(0.5$ $\mathrm{mL}$ per $\mathrm{cm}^{2}$ ) were contacted with the pristine PVDF and the composite films for 60 min at room temperature. The excess bacterial suspension was discarded. The films were washed entirely by using sterile $0.9 \%$ saline. The composite films were put into a $50 \mathrm{~mL}$ tube with $10 \mathrm{~mL}$ of sterile $0.9 \%$ saline and ultrasonicated for $10 \mathrm{~min}$ to remove the bacteria cells from the composite film. ${ }^{15}$ After that, bacteria cells were put on LB agar plates and incubated for $12 \mathrm{~h}$ at $37^{\circ} \mathrm{C}$.

To obtain the SEM images of bacterial on the composite films surface, Escherichia coli suspensions were exposed to the films as above. After contacting the cells with the composite films, the films were washed by using sterile $0.9 \%$ saline solution and fixed with Karnovsly's fixative (2.5 wt\% glutaraldehyde in $0.2 \mathrm{M}$ PBS buffer) for $3 \mathrm{~h}$. Samples were the $\mathrm{n}$ dehydrated by a sequential immersion in water:ethanol $(30,50,60,70,80,90,100 \%)$, and left to dry for $12 \mathrm{~h}$ in a desiccators below $30^{\circ} \mathrm{C}$. After that, samples need to be sputter-coated by using platinum and imaged by SEM. 


\subsubsection{Filtration module setup and permeation test}

The membrane was fixed on the flat sheet membrane $(5 \mathrm{~cm} \times 5 \mathrm{~cm}$, effective flow area is $5.25 \mathrm{~cm}^{2}$ ). The filtration module was set up as shown in Figure 5-1. The membrane was operated with in cross-flow mode, and the flow rate needs to be controlled at $1.0 \mathrm{~L} / \mathrm{min}$. DI $\mathrm{H}_{2} \mathrm{O}$ was filtrated through the module for $30 \mathrm{~min}$ before measuring the flux. Transmembrane pressure (TMP) was monitored by a pressure gauge, which was fixed at $\sim 1$ bar. The permeation test for the pristine PVDF, GOPVDF and GOQDs-PVDF membrane was operated under the same conditions.

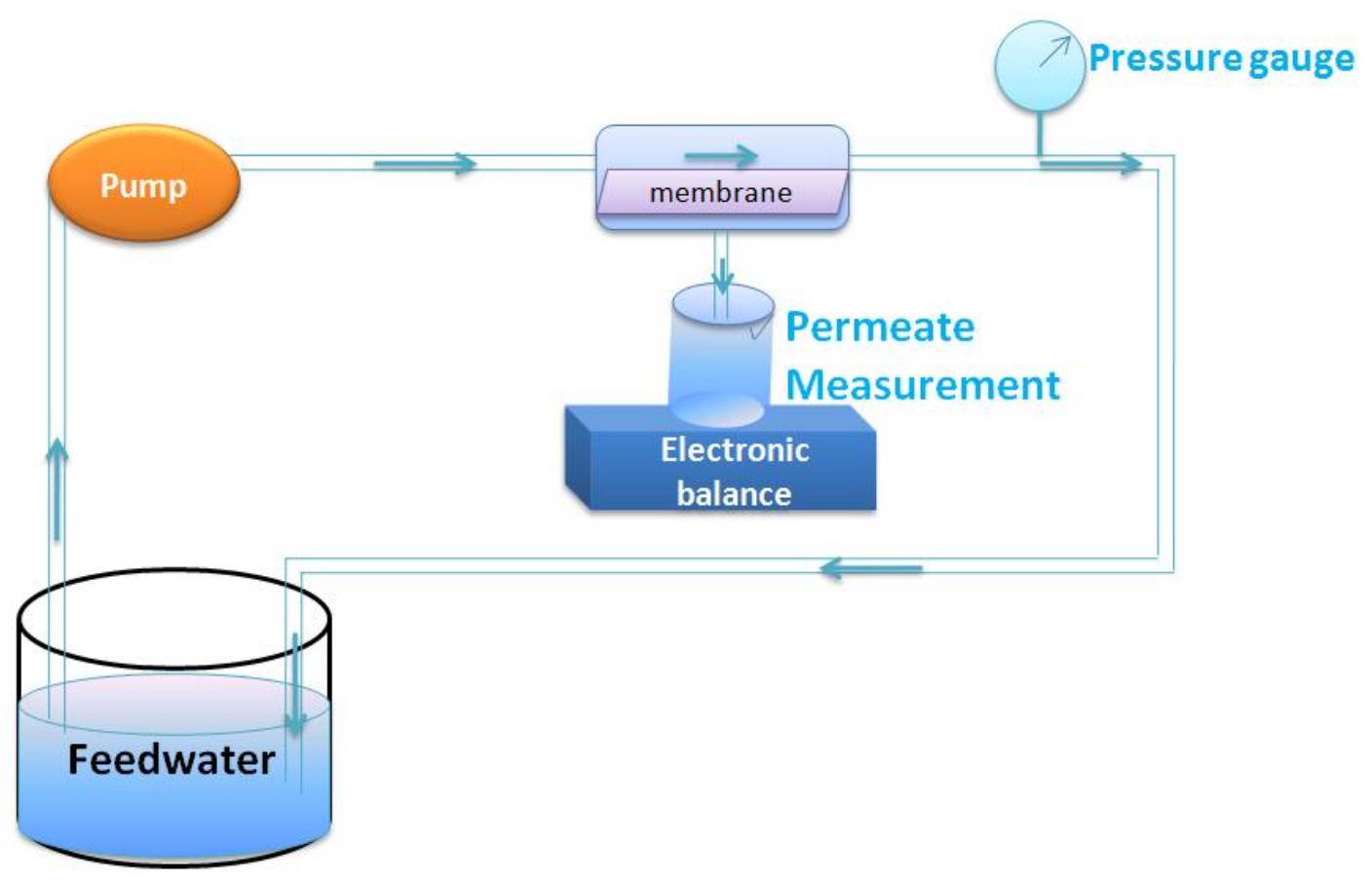

Figure 5-1 Schematic diagram of the batch experiment setup for filtration test.

\subsubsection{Filtration test of GOQDs-PVDF membrane using bacterial feedwater}

The E. coli bacterium was cultured in Luria-Bertani broth $(5 \mathrm{~g} / \mathrm{l}$ of yeast extract, $10 \mathrm{~g} / \mathrm{l}$ of tryptone and $10 \mathrm{~g} / \mathrm{l} \mathrm{NaCl}$ ) at $37^{\circ} \mathrm{C}$ for $24 \mathrm{~h}$. About $10^{9}$ colony forming units (CFU) per ml can be obtained. The bacterial cells were separated from the suspension by centrifugation, and then washed and put in a composite solution of glucose (1 wt \%) and HEPES buffer $(10 \mathrm{mM})$, and the final concentration of bacterial as the 
feedwater is approximately $10^{6}$ CFU. Before the multi-cycle operations, the membranes were flushed using pure water for $20 \mathrm{~min}$, then the feedwater was changed to use E. coli solution. The filtration test for the pristine PVDF, GO-PVDF and GOQDs-PVDF membrane was operated under the same conditions $(1.0 \mathrm{~L} / \mathrm{min}$ of flux rate, 1 bar of permeation pressure). Before examining the membrane by FESEM, the samples were performed in freeze dryer at $55^{\circ} \mathrm{C}$ for $12 \mathrm{~h}$.

\subsubsection{Oxidative stress of GOQDs-PVDF/GO-PVDF membrane assay}

The concentration of thiols in GSH can be evaluated by the Ellman's assay.(225,237) The pristine PVDF, GO-PVDF and GOQDs-PVDF $\left(5 \times 5 \mathrm{~mm}^{2}\right)$ in 50 $\mathrm{mM}$ bicarbonate buffer was mixed into $300 \mu \mathrm{l}$ of GSH. All samples were tested in triplicate. After incubation for $1 \mathrm{~h}, 800 \mu \mathrm{l}$ of $0.05 \mathrm{M}$ Tris- $\mathrm{HCl}$ and $20 \mu \mathrm{l}$ of 5,5'dithio-bis-(2-nitrobenzoic acid) (DNTB, Sigma-Aldrich) were added into the mixtures. A $300 \mu$ aliquot of solution was then placed in a 24-well plate. By using Benchmark Plus microplate spectrophotometer, the absorbance at $412 \mathrm{~nm}$ was monitored. GSH solution without membrane was used as a negative control. GSH oxidization by $\mathrm{H}_{2} \mathrm{O}_{2}(1.0 \mathrm{mM})$ was tested as a positive control.

\subsection{Results and discussion}

\subsubsection{Fabrication and characterization of GOQDs, GO, GOQDs-PVDF and GO-PVDF}

GOQDs were fabricated from carbon black $(\mathrm{CB})$ via a hydro-thermal oxidation treatment.(211) Typically, XC-72 CB was refluxed in concentrated $\mathrm{HNO}_{3}$ solution (65 wt $\%$ ) at $110{ }^{\circ} \mathrm{C}$ for about $24 \mathrm{~h}$. After cooling to room temperature, the mixture was diluted with plenty of deionized (DI) water and then filtered with a $10 \mathrm{kDa}$ molecular weight cut-off membrane. The percolate was subsequently dried in oven at $60{ }^{\circ} \mathrm{C}$. The as-obtained GOQD sample was characterized by transmission electron 
microscopy (TEM). As can be seen from Figure 5-2a\&b, the as-prepared GOQDs exhibit an average diameter of $\sim 5.5 \mathrm{~nm}$ with a relatively narrow size distribution. A high-resolution TEM (HRTEM) image (Figure 5-2c) indicates that the GOQDs possess good crystallinity with a lattice spacing of $0.241 \mathrm{~nm}$, which corresponds to (1120) lattice fringe of graphene. Furthermore, atomic force microscopy (AFM) observation reveals, that the topographic height of GOQDs is about $0.7 \mathrm{~nm}$ (Figure 5-2d), which is consistent with the thickness of monolayer graphene. The GOQD sample is further investigated by the C 1s X-ray photoelectron spectroscopy (XPS) and Fourier transform infrared spectroscopy (FTIR). As observed in Figure 5-2e, the XPS spectrum of the GOQD revealed a strong O signal compared to that of carbon black, suggesting the presence of abundant oxygenated groups in GOQDs after acidoxidation treatment. Quantitative analysis of the XPS spectrum determined that the atomic ratios $(\mathrm{C} 1 \mathrm{~s}) /(\mathrm{O} 1 \mathrm{~s})$ of GOQDs and GO were 2.26 and 2.18, respectively (Figure 5-2e, 5-3b), which is consistent with the reported $\mathrm{C} / \mathrm{O}$ ratio of graphene related materials.(234) For the high-resolution XPS C 1s spectra, the GOQD sample presented two additional signals at 286.2 and $288.6 \mathrm{eV}$, respectively, corresponding to hydroxyl groups and carboxyl groups (Figure 5-2f). The presence of oxygencontaining functional groups, including carboxyl, carbonyl and hydroxyl groups, was further confirmed by the FTIR spectrum (Supporting information Figure 5-4a). Raman spectrum exhibits that two characteristic signals of graphene, D band at 1359 $\mathrm{cm}^{-1}$ and $\mathrm{G}$ band at $1599 \mathrm{~cm}^{-1}$ (Figure 5-4b), of the GOQDs. The UV-vis absorption spectrum shows that the GOQDs possess a broad absorption below $580 \mathrm{~nm}$, and the emission wavelengths become excitation-dependent and exhibit a red shift when increasing excitation wavelengths, probably due to various emissive sites of the synthesized GOQDs (Figure 5-4c). 
a
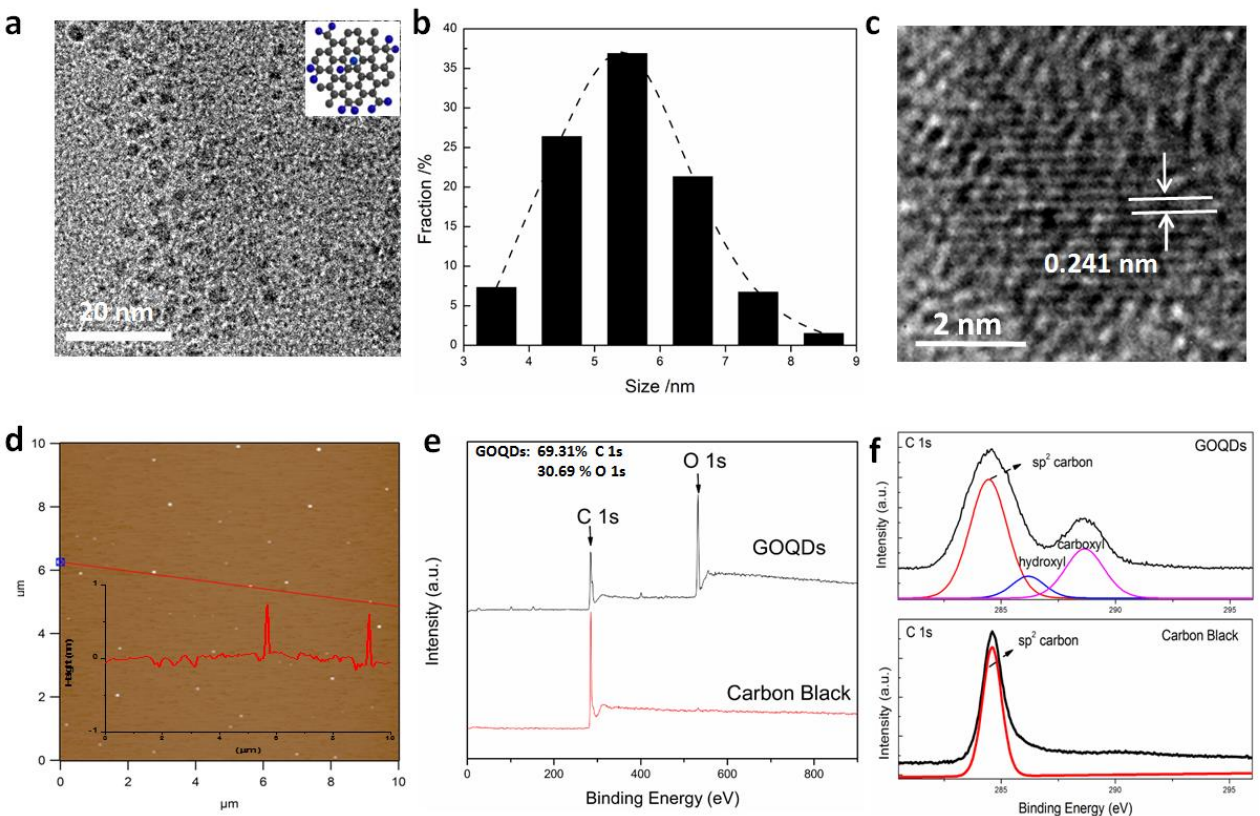

Figure 5-2. (a) TEM image and (b) Size distribution of GOQDs; (c) Highresolution TEM (HRTEM) and (d) AFM images of GOQDs, inset shows the height profile along the red line; (e) Survey and (f) high-resolution XPS spectra of C 1s for GOQDs and carbon black.
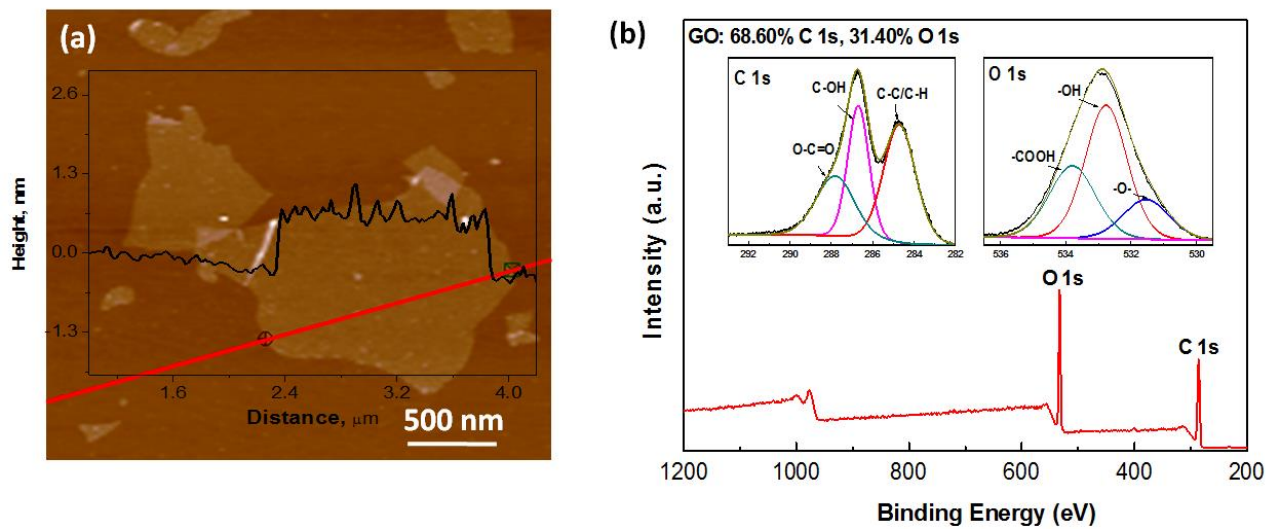

Figure 5-3. (a) Tapping mode AFM image of GO sheets, inset shows the height profile along the red line; (b) High-resolution XPS spectra of GO. 

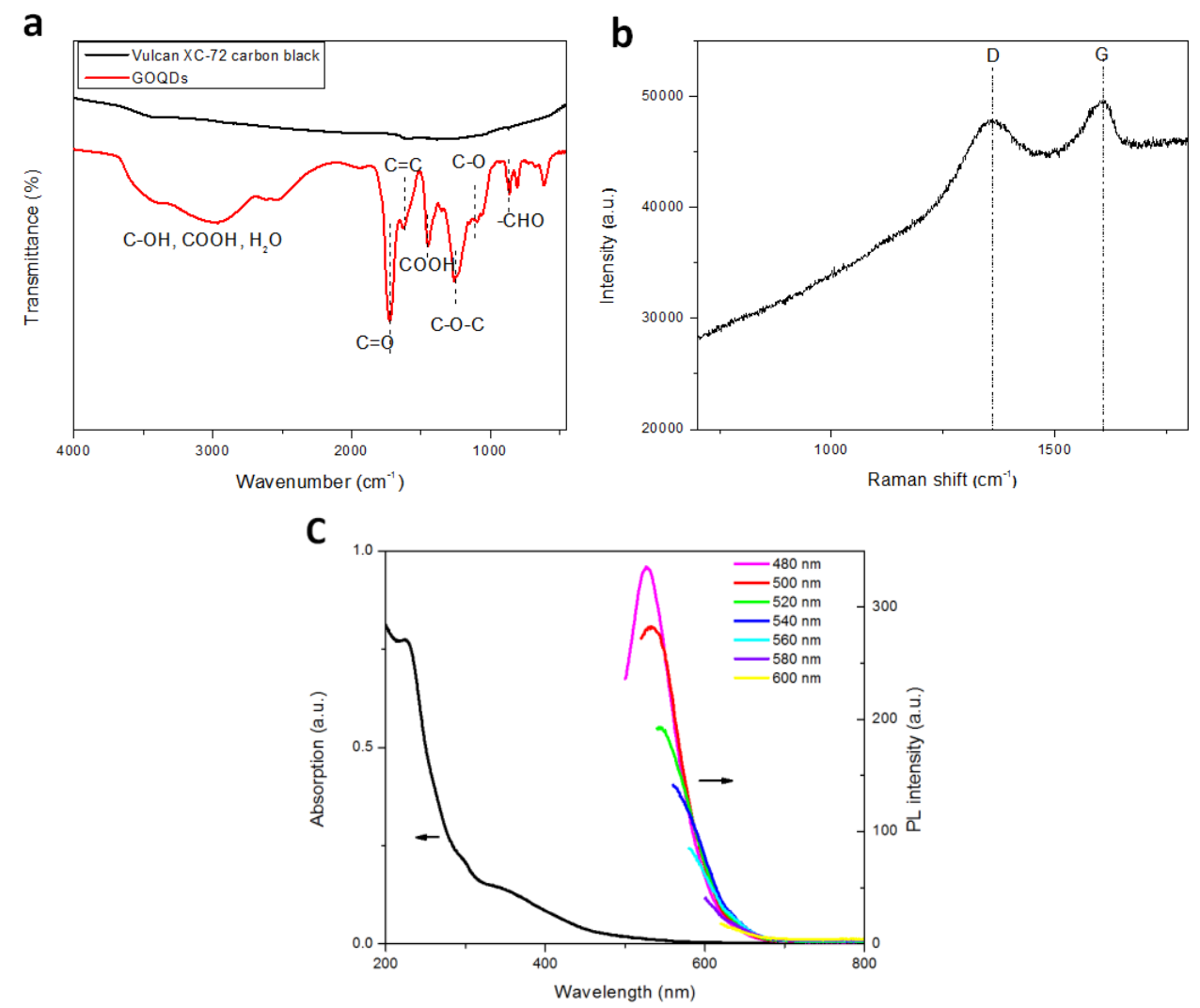

Figure 5-4. (a) FTIR spectra of carbon black and GOQDs; (b) Raman spectrum of GOQDs; (c) PL emission spectra and UV-vis absorption spectra of GQDs solution.

The synthetic procedure for the GOQDs functionalized PVDF membrane was depicted in Figure 5-5. The PVDF membrane surface was firstly hydroxylated via helium plasma induced grafting of polyethylene glycol (PEG). The PEG-grafted PVDF membrane surface was then treated with 3-Aminopropyl-trimethoxysilane (APTMS) to introduce amine groups, which allows the covalent linkage of GOQDs through the formation of covalent bonds between the carboxylic groups on GOQDs and the amine groups on the PVDF surface in the presence of ethyl(dimethylaminopropyl) carbodiimide/N-hydroxysuccinimide (EDC/NHS). The overall process led to the formation of GOQDs functionalized PVDF membrane. 


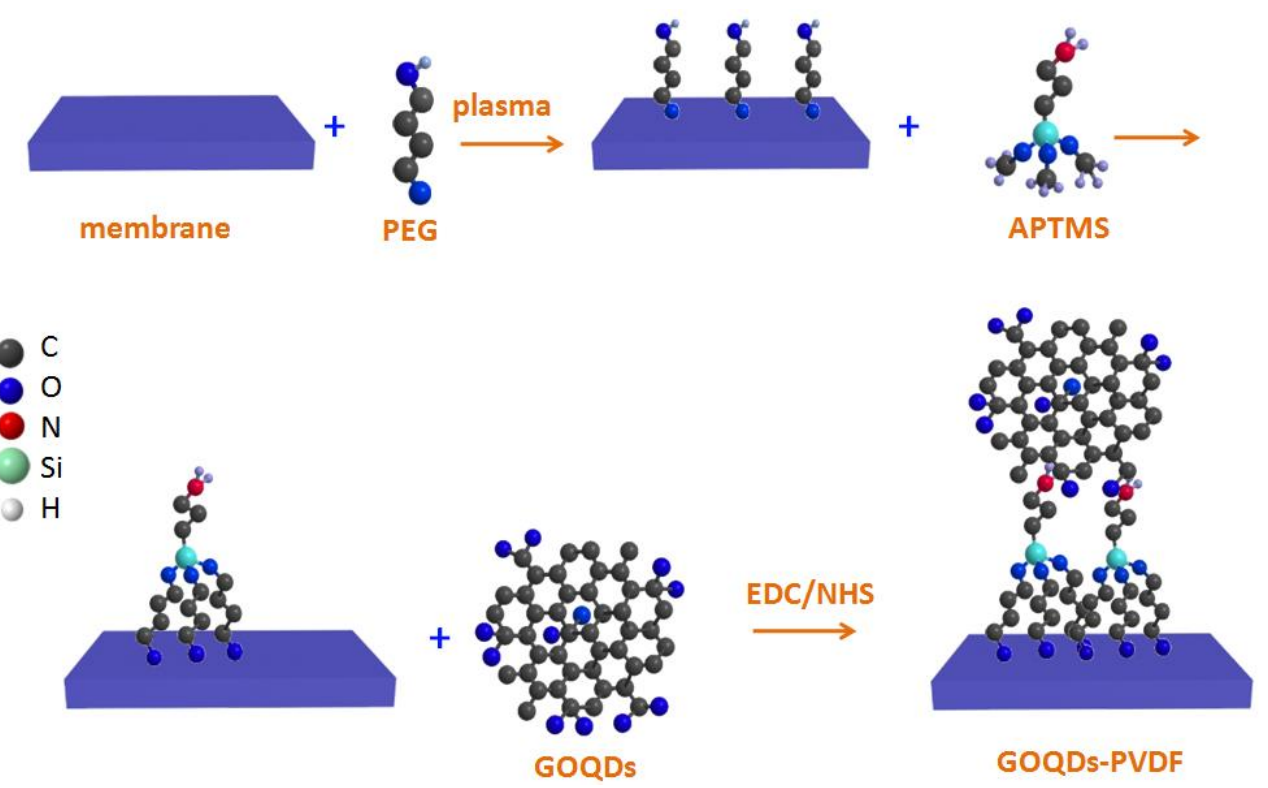

Figure 5-5. Schematic illustration for covalent immobilization of GOQDs onto the PVDF membrane surface.

The original and functionalized PVDF membranes (the average pore diameter, $0.22 \mu \mathrm{m})$ were characterized by scanning electron microscopy (SEM). It is evident that the original PVDF membrane displays a porous structure with a smooth interior surface (Figure 5-6a, b). In contrast, after helium plasma treatment with PEG, the interior surface for the plasma-treated membrane becomes rough, as shown in Figure 5-6c, while the membrane after the subsequent modification of APTMS remains smooth (Figure 5-6d), similar with that of original PVDF membrane. Contrary to the smooth surface morphology in Figure 5-6d, Figure 5-6e shows the uniform coating of nanoparticles on the PVDF surface, suggesting the successful immobilization of GOQDs. Notably, the GOQD modified PVDF membrane still retains a good structural integrity even after subjected to ultrasonication treatment for $15 \mathrm{~min}$. The good stability should be resultant from the strong covalent linkage formed between the abundant carboxyl groups on GOQDs and the amino groups of APTMS modified PVDF membrane. 

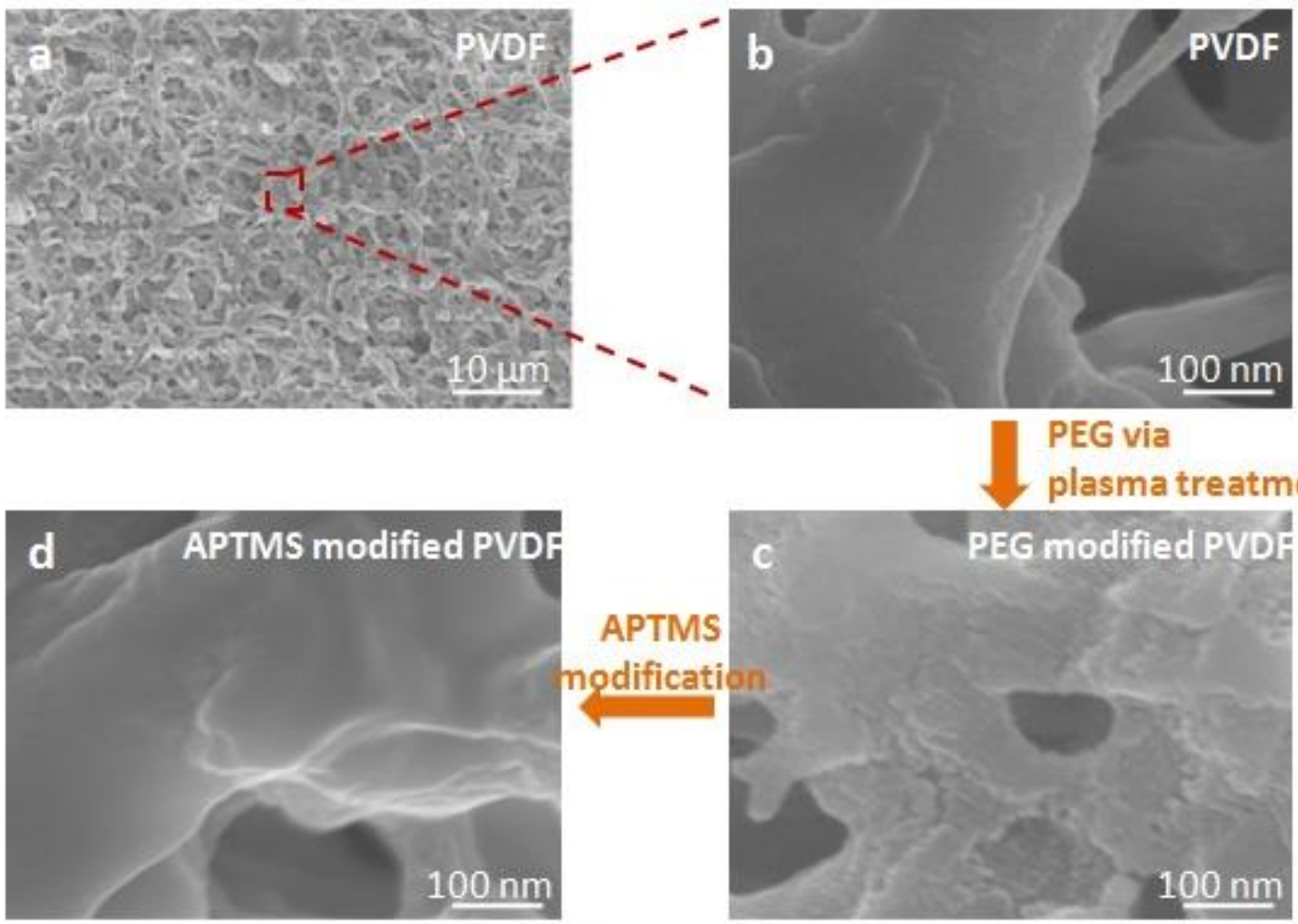

GOQDs, EDC/NHS
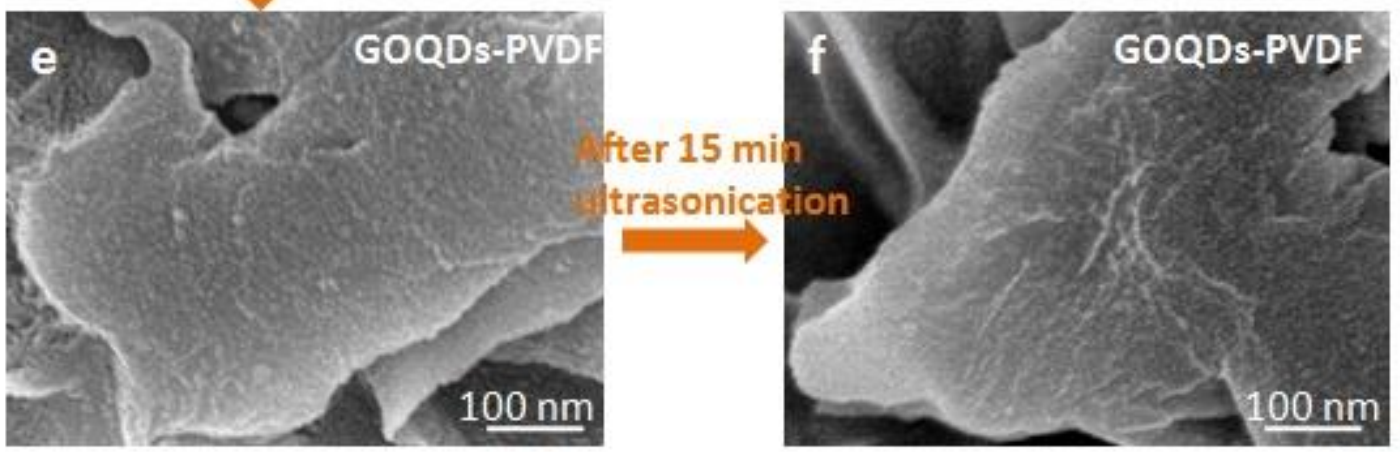

Figure 5-6. SEM images of (a, b) pristine PVDF, (c) PEG modified PVDF, (d) APTMS modified PVDF, (e) GOQDs-PVDF and (f) GOQDs-PVDF hybrid membrane after 15 min ultrasonication treatment.

The covalent immobilization of GOQDs on the PVDF membrane surface is further supported by ATR-FTIR spectra (Figure 5-7). As can be seen, the original PVDF membrane shows a strong band at $1402 \mathrm{~cm}^{-1}$ corresponding to the stretching vibration of $\mathrm{C}-\mathrm{H}$ bonds, while the bands located at $1275 \mathrm{~cm}^{-1}$ and $1178 \mathrm{~cm}^{-1}$ are attributed to the vibration of C-F bonds. In comparison with the spectrum of the pristine PVDF membrane, the symmetrical and asymmetrical stretching of C-F bonds 
$\left(1275 \mathrm{~cm}^{-1}\right.$ and $1178 \mathrm{~cm}^{-1}$, respectively) and the vibration of $\mathrm{C}-\mathrm{H}$ bond $\left(1042 \mathrm{~cm}^{-1}\right)$ became weaken remarkably after the PEG grafting treatment, attributed to the dehydrofluorination. The adsorption band (around $3400 \mathrm{~cm}^{-1}$ ) is ascribed to the hydroxyl groups stretching, which appears after the treatment. It is proposed that the dehydrogenation of PEG and dehydrofluorination of PVDF under $\mathrm{He}$ plasma environment generated radicals, which then underwent in the reaction with the facilitating the formation of hydroxyl groups on the PVDF membrane surface.(210,238) This result confirms a successful fabrication of PEG-modified PVDF membrane. Compared with the pristine PVDF membrane, a new band appears at $1659 \mathrm{~cm}^{-1}$ in the FTIR spectrum of the APTMS modified PVDF membrane, attributed to the stretching vibration of $-\mathrm{NH}_{2}$. This result confirms the effective grafting of APTMS on the PVDF surface. After the GOQD immobilization, the signal of $-\mathrm{NH}_{2}$ band at $1659 \mathrm{~cm}^{-1}$ is attenuated weak and a new band is presented at 1387 $\mathrm{cm}^{-1}$ in the FTIR spectrum of GOQDs-PVDF, which is attributed to the stretching vibration of amide bonds (-NH-CO-). This suggests the presence of covalent linkage of GOQDs with the PVDF membrane. Furthermore, the strong band at $3400 \mathrm{~cm}^{-1}$ should arise from the -OH groups of GOQDs.

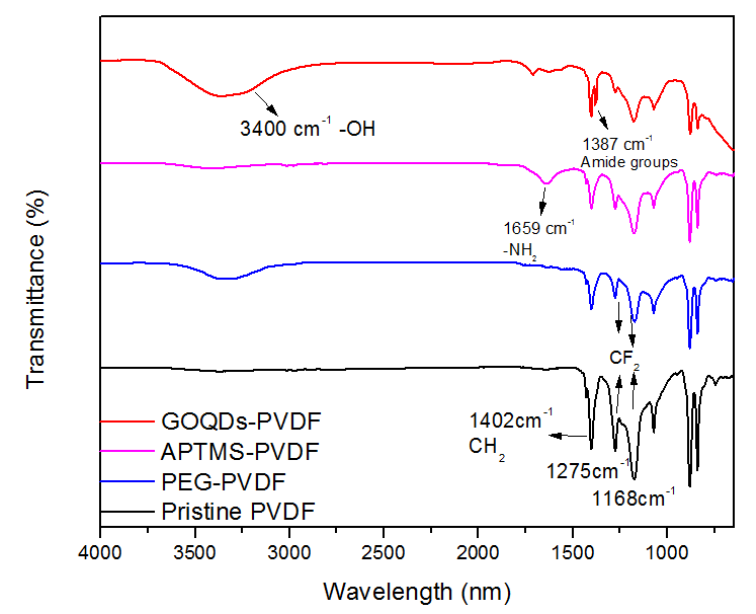

Figure 5-7. ATR-FTIR spectra of pristine PVDF, PEG modified PVDF, APTMS modified PVDF and GOQDs-PVDF. 
It can also be found that the Raman spectra of GOQDs-PVDF and GO-PVDF membranes exhibit the obvious $\mathrm{G}$ band $\left(1596 \mathrm{~cm}^{-1}\right)$ and $\mathrm{D}$ band $\left(1347 \mathrm{~cm}^{-1}\right)$ of graphene structure (Figure 5-8). Therefore, the FTIR and Raman results, in conjunction with the above SEM observation, confirm the covalent decoration of GOQDs on the PVDF membrane. Through a zeta potential test, APTMS-PVDF membrane was found to have an isoelectric point of 9.8 and was positively charged due to the protonation of the amine group. However, the deprotonation of carboxyl group results in the GOQDs-PVDF or GO-PVDF membrane to be negatively charged in a wide $\mathrm{pH}$ range from 3.8 to 10.2 (Figure 5-9).

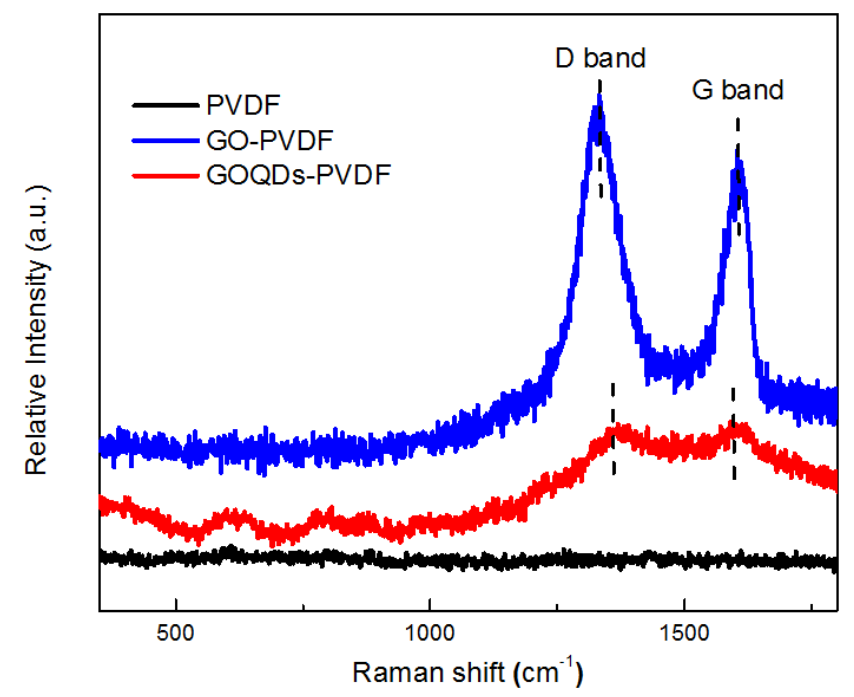

Figure 5-8. Raman spectra of pristine PVDF, GO-PVDF, GOQDs-PVDF membranes. 


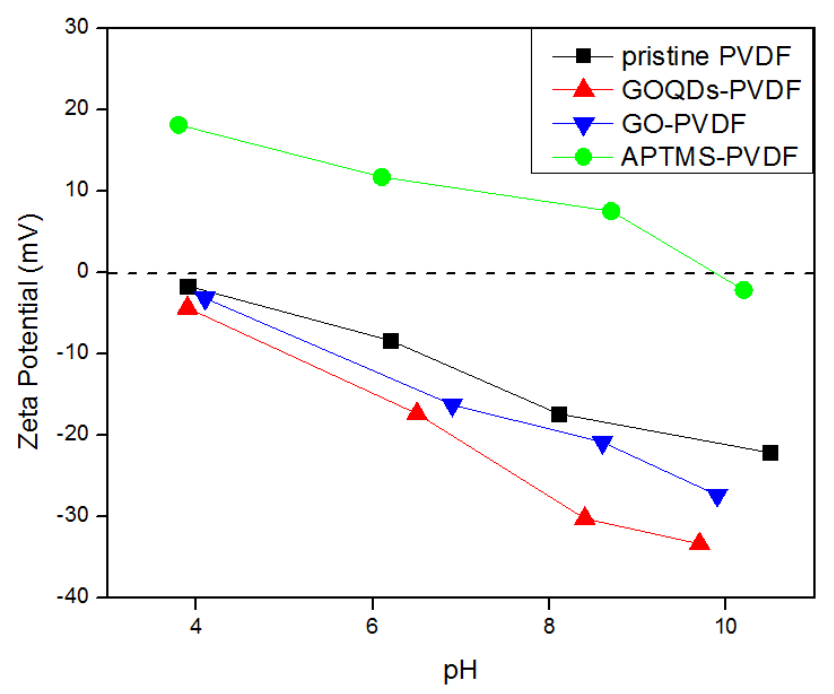

Figure 5-9. Zeta-potential of pristine PVDF, APTMS modified PVDF, GOQDsPVDF and GO-PVDF membranes.

\subsubsection{Membrane properties of pristine PVDF, GO-PVDF and GOQDs-PVDF} membranes
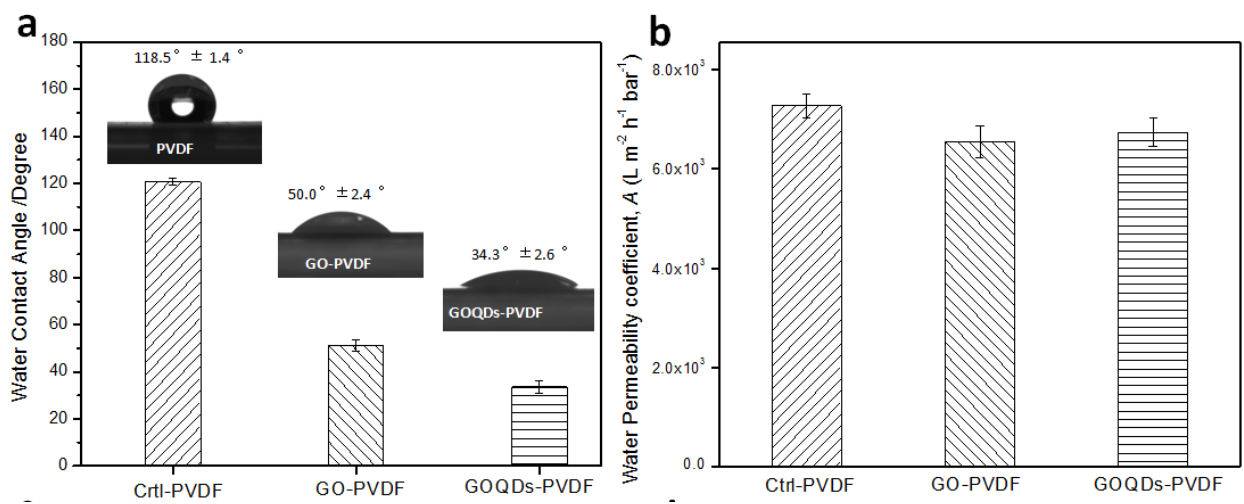

Figure 5-10 Membrane properties of pristine PVDF, GO-PVDF and GOQDsPVDF membranes. (a) Water contact angle. (b) Water permeability coefficient, A.

Membrane propensity for antibiofouling depends on the physicochemical properties of the membrane surface, and a key property is the hydrophilicity of the surface. The hydrophilicity of the membrane surface can be evaluated by measuring the water contact angle (CA). The natural wettability of the membrane surface can be reflected by the CA parameter measured immediately through water droplets on the material surface.(239) The pristine PVDF membrane showed a relatively 
hydrophobic surface with a CA of $118.5^{\circ}$. After modified with 0 D GOQDs, the CA values decreased dramatically to $34.3^{\circ}$, which is also smaller than that of $2 \mathrm{D}$ GO nanosheet (Figure 5-3) modified PVDF (GO-PVDF) membrane $\left(50.0^{\circ}\right)$ produced by the similar procedure (Figure 5-10a). The significantly-improved hydrophilicity for the GOQDs modified PVDF membrane should be due to the presence of abundant oxygen-containing functional groups on GOQDs which are evenly distributed on the membrane surface. Figure 5-10b presents the flux test results of the pristine PVDF membrane, the GO-PVDF membrane, and the GOQDs-PVDF membrane. It is observed that after the modification of $0 \mathrm{D}$ GOQDs sheets, the pure water permeability coefficient decreases slightly to $(6.83 \pm 0.28) \times 10^{3} \mathrm{~L} \mathrm{~m}^{-2} \mathrm{~h}^{-1} \mathrm{bar}^{-1}$, relative to that of the pristine PVDF membrane, $(7.07 \pm 0.24) \times 10^{3} \mathrm{~L} \mathrm{~m}^{-2} \mathrm{~h}^{-1} \mathrm{bar}^{-1}$, while the decrease in flux is lower than that of 2 D GO nanosheets modified PVDF membrane $\left((6.55 \pm 0.32) \times 10^{3} \mathrm{~L} \mathrm{~m}^{-2} \mathrm{~h}^{-1} \mathrm{bar}^{-1}\right)$. This is attributed to the uniform surface coverage of the small-sized GOQDs on the membrane surface in comparison with large-sized 2 D GO nanosheets (Figure 5-11).

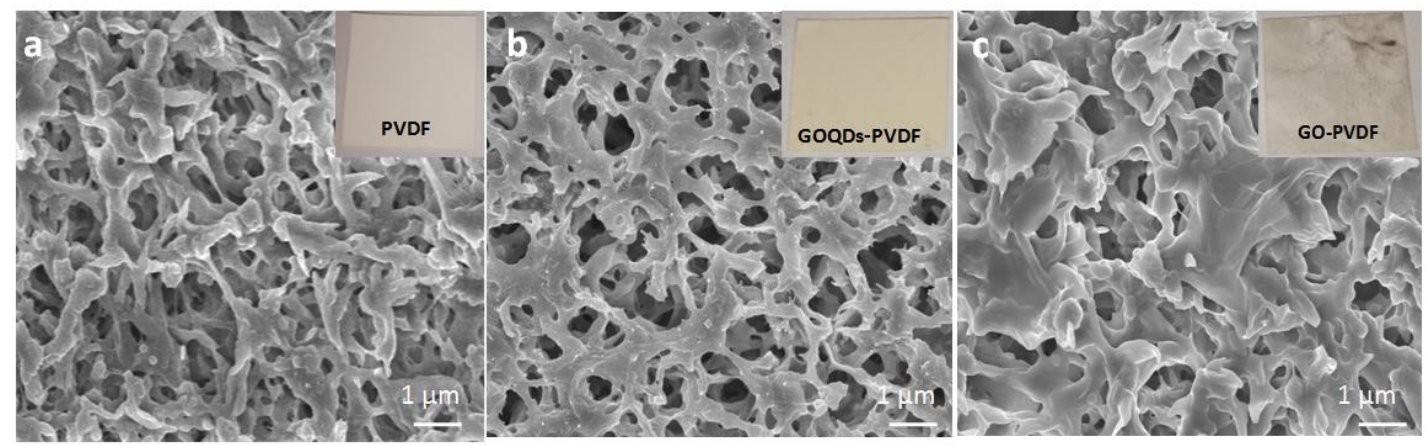

Figure 5-11. SEM images of PVDF (a), GO-PVDF (b), and GOQDs-PVDF (c). The upper inset are the optical images of the corresponding membranes. 


\subsubsection{Antibacterial and antibiofouling performance of GOQDs-PVDF hybrid membrane}

Bactericidal and antibiofouling properties of the GOQDs and GO modified PVDF membrane was investigated by using Escherichia coli (E. coli).(91,226) E. coli-containing feedwater was made from a neutral mixture solution of HEPES/glucose $(\mathrm{pH} 7.0)$, which can afford a nutritious environment to keep the bacteria survived during long-time filtration. Taking into account the effect of the medium on permeation, a control flux measurement was carried out for a blank PVDF membrane using the HEPES/glucose solution as a feedwater. Figure 5-12a shows that the flux drop of about $15.6 \%$ during the initial $4 \mathrm{~h}$ and a subsequent $3.2 \%$ drop after the next $16 \mathrm{~h}$. The initial decrease might be caused by the compaction of medium and the permeation of ionic species into the pores of PVDF. This implies that the medium does not affect the hydrodynamics of the membrane. In contrast, after adding E. coli to the feedwater $\left(10^{6} \mathrm{CFU}\right)$, the resulting solution can significantly affect the permeation of the PVDF membrane. As observed from Figure 5-12a, 5-12c, the flux of pristine PVDF decreased $88.4 \%$ after $12 \mathrm{~h}$ filtration, suggesting that the pure PVDF membrane can be easily biofouled. In contrast, the permeation decrease of the PEG-modified PVDF, APTMS-modified PVDF and GO-modified PVDF membranes were $79.0 \%, 76.2 \%$ and $65.7 \%$, respectively, while the flux drop of the GOQDs-PVDF membrane was only $24.3 \%$ over the same duration of filtration. These results strongly suggest that the GOQDs-modified PVDF membrane possesses a much better biofouling resistance compared to the pristine PVDF, PEG-modified PVDF, APTMS-modified PVDF and GO-PVDF membranes. More importantly, the permeation value of GOQDs-PVDF remains stable even after 10 hours. During a multi-cycle operation of the filtration with E. coli solution (Figure 5-12b), the final 
flux of GOQDs-PVDF membrane is two times larger than that of the GO-PVDF membrane, although the performances of both composite membranes can be largelymaintained after three filtration cycles. It is also observed that GOQDs-PVDF membrane can be easily cleaned and the flux can be recovered after forward flushing using DI water. However, the permeability of the pristine PVDF membrane showed a significant decrease, and after washed by DI water, it cannot be recovered to the initial value. This result indicates that the fouling resistance of the membrane can be significantly improved by the introduction of a uniform GOQDs layer which consists of abundant oxygen-containing functional groups on the membrane surface.(240)
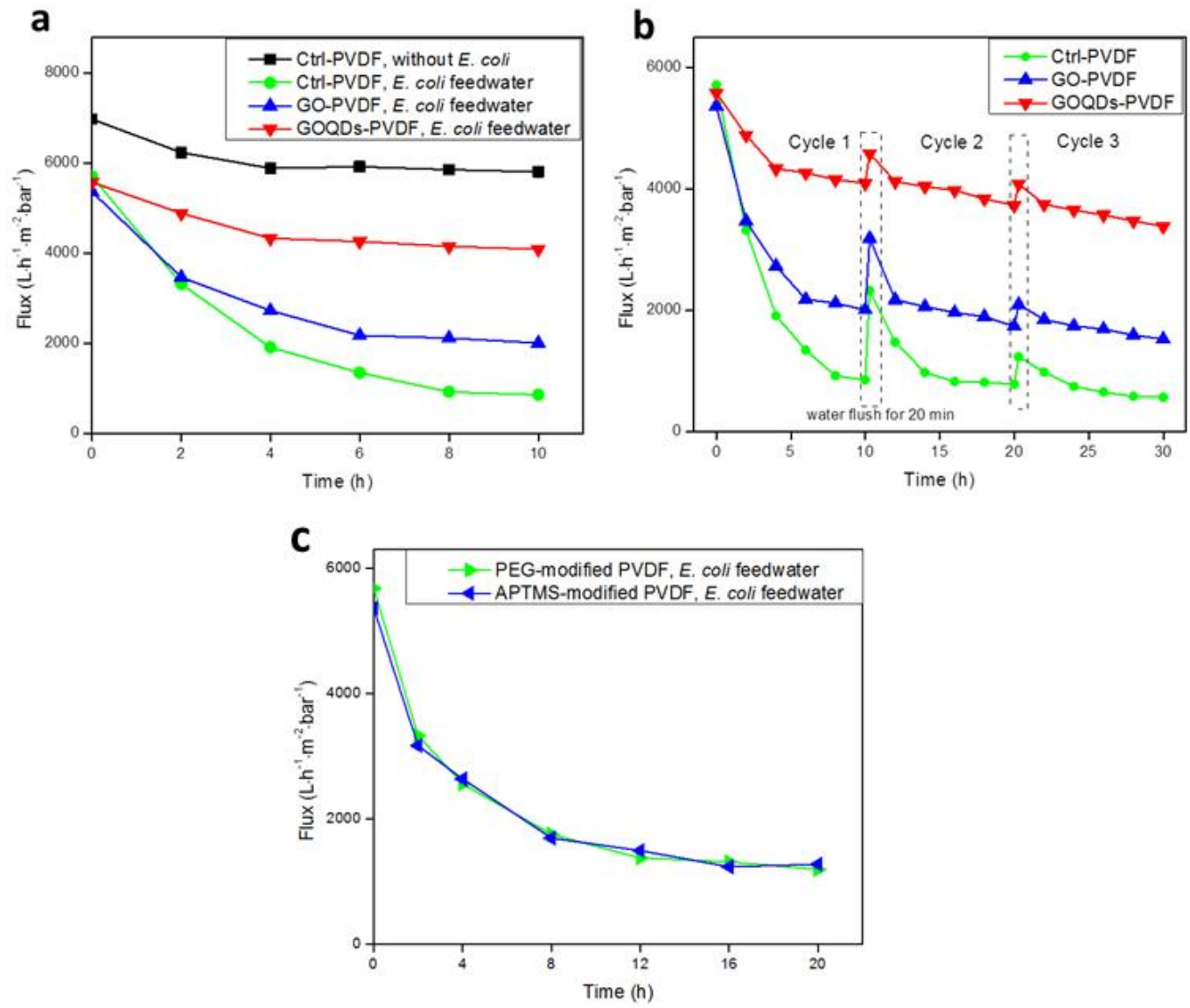

Figure 5-12 (a) The water permission by using $E$. coli-containing feedwater during a $20 \mathrm{hr}$ continuous filtration test. (b) The flux of PVDF membrane, GOPVDF membrane and GOQDs-PVDF membrane with three cycles of $E$. colicontaining solution filtration. Before multi-cycle filtration operations, the membranes were forward flushed using pure water for $20 \mathrm{~min}$, and then the feedwater was changed to use E. coli solution. (c) The flux change of PEG- 
modified PVDF, APTMS-modified PVDF membranes using E. coli-containing feedwater during a $20 \mathrm{hr}$ continuous filtration test.

To provide deeper insight into the antibiofouling behavior of the functionalized membrane, the interaction between bacteria and the membrane was further investigated. The proliferation activities of E. coli and Staphylococcus aureus ( $S$. aureus) on the pristine and modified PVDF membranes were measured via a plate counting method. After attached to the GOQDs-PVDF membrane for $1 \mathrm{~h}$, the metabolic activity of $E$. coli cells was reduced by $88.9 \%$ (Figure 5-13), while the decrease of metabolic activity of E. coli on the 2 D GO modified PVDF membrane was $69.2 \%$ over the same period of time $(1 \mathrm{~h})$, similar with a previously reported GO functionalized polyamide membrane.(226)
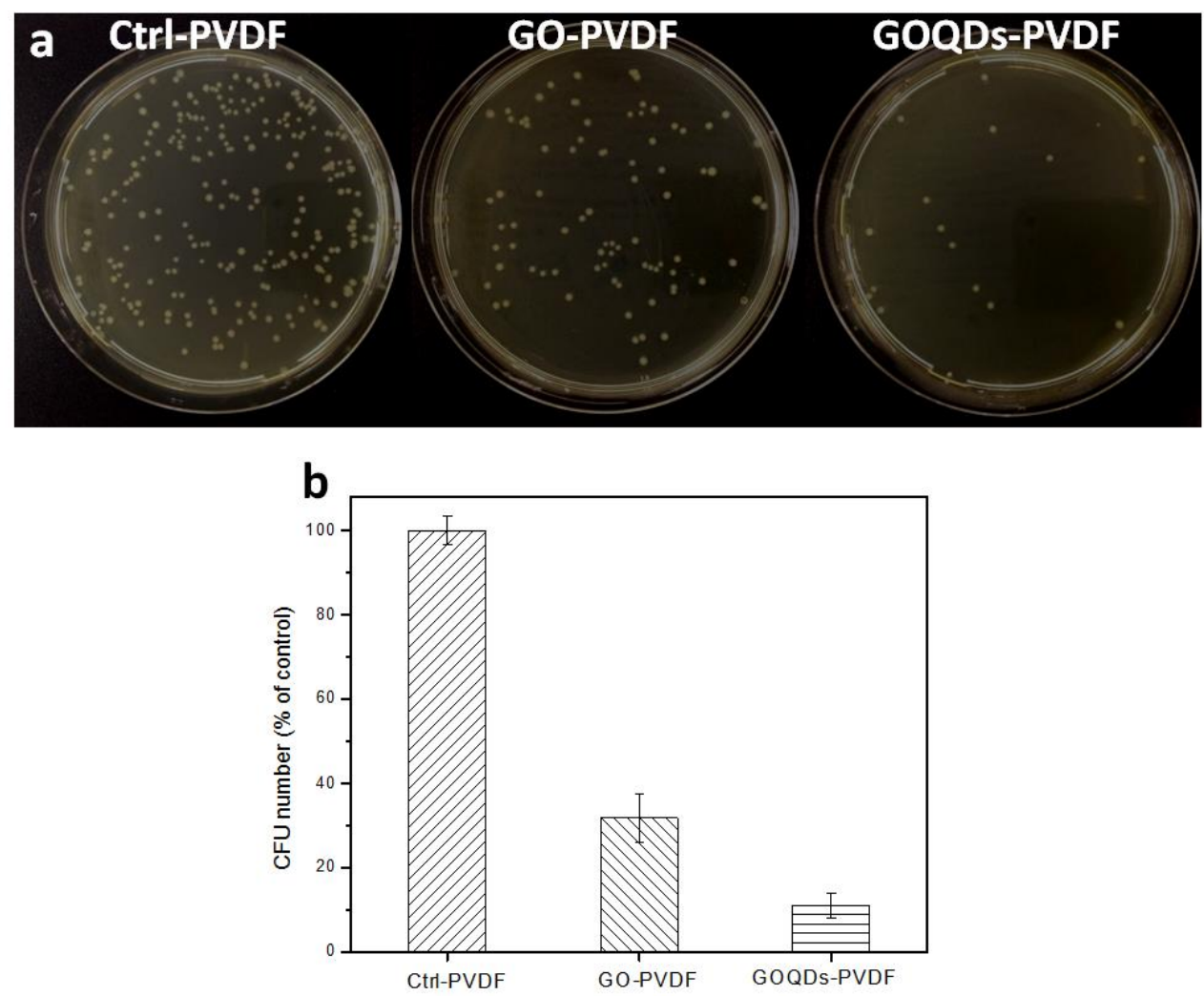

Figure 5-13 Graphene functionalized membranes reduce the number of $E$. coli cells colonies. (a) $E$. coli suspensions were exposed simultaneously to pristine PVDF, GO-PVDF and GOQDs-PVDF for $1 \mathrm{~h}$. (b) The difference of CFU for the different membranes; CFU was determined by standard plate count method. 
S. aureus cells colonies were exposed simultaneously to pristine PVDF, GOPVDF and GOQDs-PVDF for $1 \mathrm{~h}$, which showed a $77.9 \%$ and $53.7 \%$ decrease of metabolic activity of $S$. aureus cells on the GOQDs-PVDF membrane and GO modified PVDF membrane, respectively (Figure 5-14). In addition, the bacterial inactivation of the current 0 D GOQDs modified PVDF membrane is observed to be more effective than that of an $1 \mathrm{D}$ SWNTs anchored polyamide membrane, which shows $60 \%$ inactivation of bacteria in 1 h.(224) Thus, the superior antimicrobial activity of GOQDs-PVDF relative to GO-PVDF should be attributed to the uniform dispersion of GOQDs with a larger fraction of active edges on the membrane surface, enabling the direct contact with and/or physical piercing to the bacterial cells.
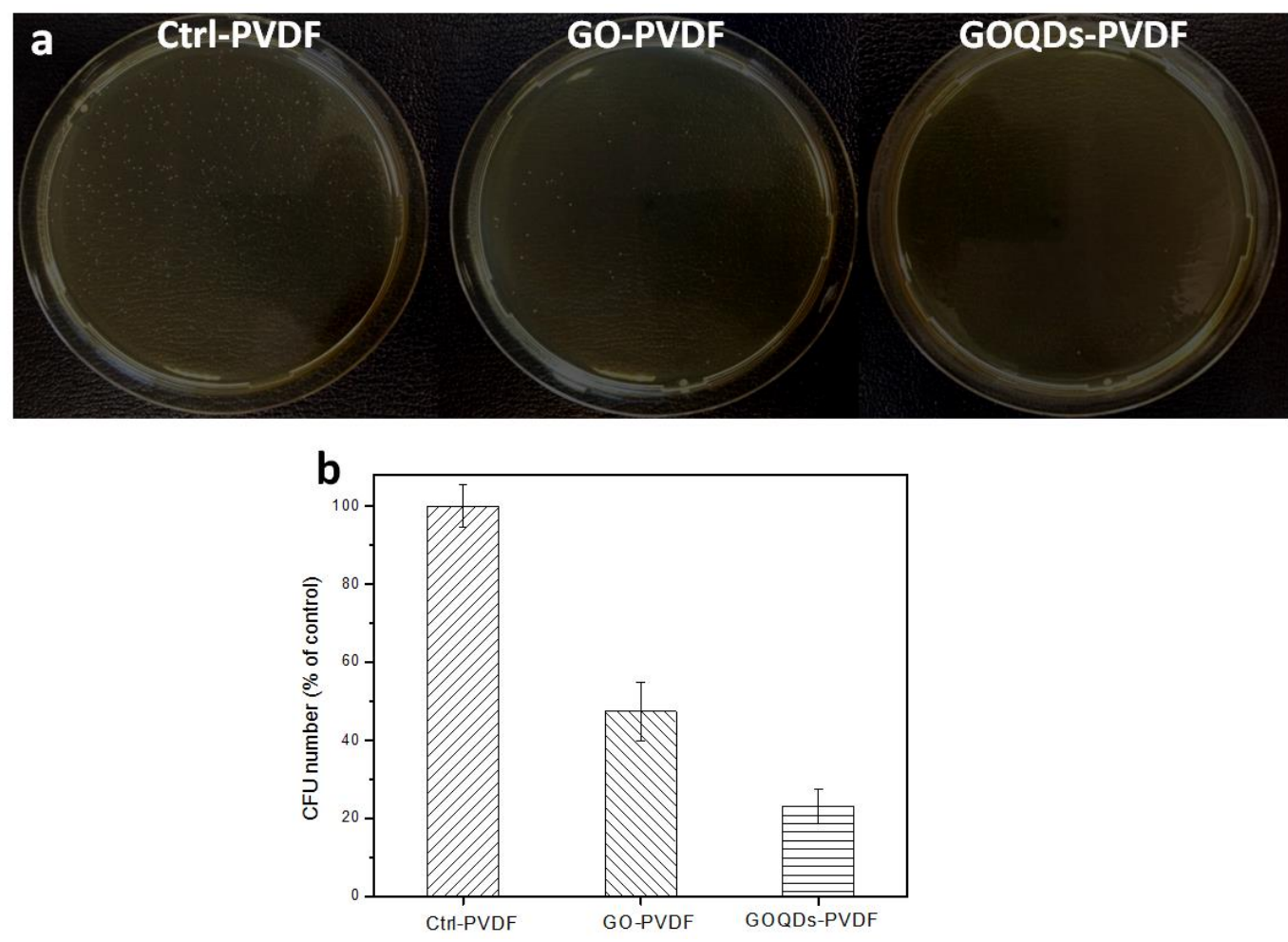

Figure 5-14. Graphene functionalized membrane reduce the number of $S$. aureus cells colonies. (a) $S$. aureus suspensions were exposed simultaneously to pristine PVDF, GO-PVDF and GOQDs-PVDF for $1 \mathrm{~h}$. (b) The difference of CFU for the different membranes; CFU was determined by standard plate count method. 
By tuning of GOQDs concentration in the precursor solution, the effect of the GOQDs loading on the PVDF membranes on the antibacterial and the permeation were also studied. As shown in Figure 5-15, the loading amount of GOQDs on the surface of membrane increased gradually with the increase of the concentration of aqueous GOQDs solution, and a full coverage of GOQDs on the surface was achieved at a specific concentration $(\sim 1.0 \mathrm{mg} / \mathrm{ml})$. Using the same plate counting method, the proliferation activity of $E$. coli on the composite membranes with different densities of GOQDs was measured. The CFU numbers of bacterial cells were decreasing with increasing the concentration of GOQDs. Simultaneously, the final flux of the GOQDs functionalized membranes after $10 \mathrm{~h}$ permeation test with bacterium-containing feedwater were significantly increased when increasing the concentration of GOQDs. It is noted that the antibacterial activity and biofouling resistance of the GOQDs-PVDF membrane was enhanced with increasing the concentration of GOQDs from 0 to $1 \mathrm{mg} / \mathrm{ml}$. When the GOQDs concentration was further increased, the membrane surface exhibited the formation of a multilayer of particles due to the aggregation of excess GOQDs, and the antimicrobial activity and biofouling resistance showed no significant enhancement (Figure 5-15f), which can be attributed to the complete coverage of GOQDs on the membrane surface. The results indicate that the loading amount of GOQDs on the membrane surface can be well controlled to achieve the optimal water permeation, antimicrobial and antibiofouling activity of composite membrane. 

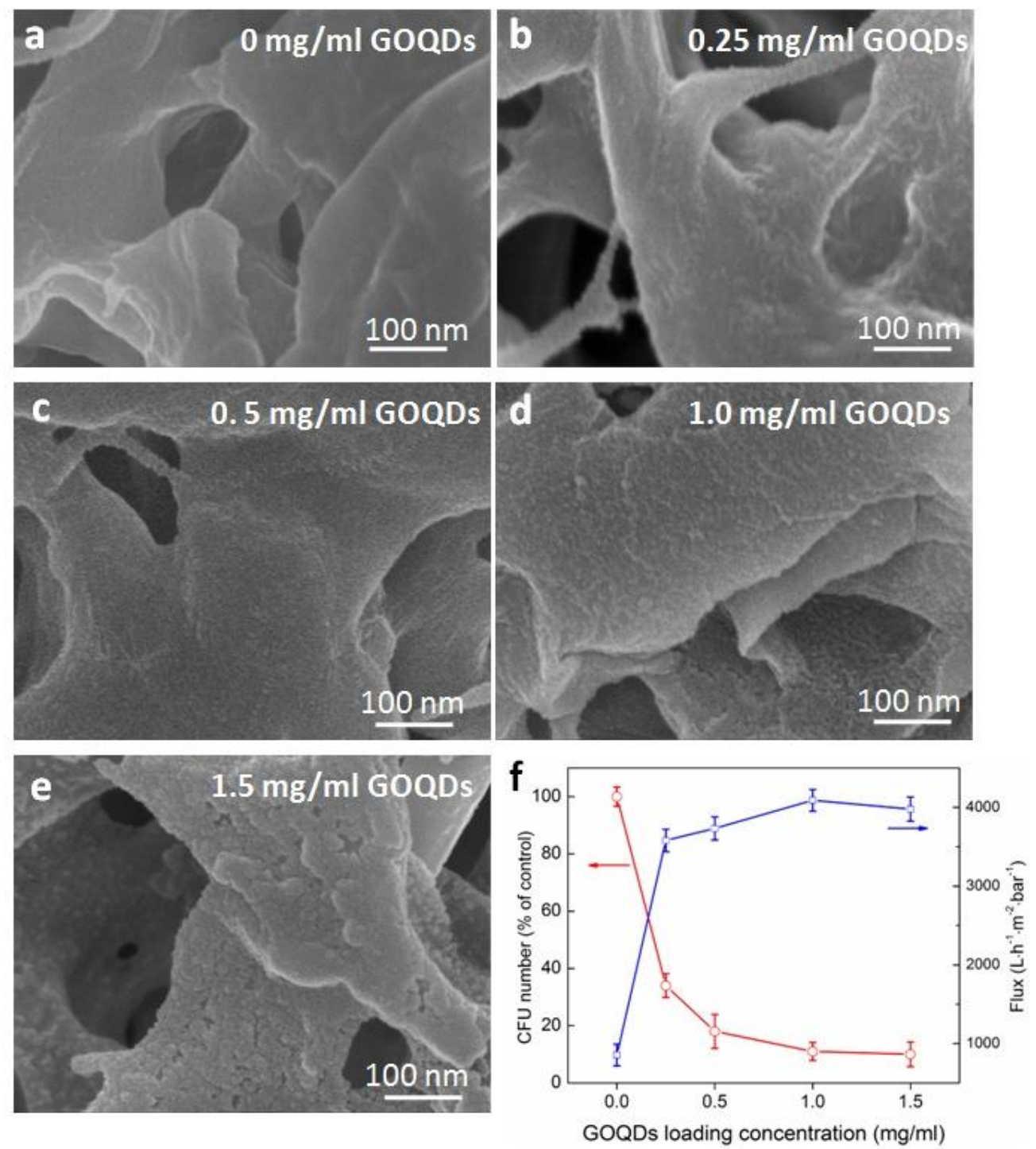

Figure 5-15 (a-e) SEM images of GOQDs-PVDF with different loadings amount of GOQDs, which are prepared by changing the concentration of GOQDs in the aqueous solution. (f) The loss number of CFU (after $1 \mathrm{~h}$ contact) and the flux change of GOQDs-PVDF (after $10 \mathrm{~h}$ filtration) with different loading amount of GOQDs.

\subsubsection{Bactericidal mechanism of GOQDs-PVDF hybrid membrane}

In order to elucidate the antibacterial mechanism of the GOQDs-PVDF membrane, SEM observation was used to provide visual information of the bacterial content on the membrane surface. Most of E. coli cells on the GOQDs-PVDF membrane surface have compromised structure (Figure 5-16c, d), while the cells attached to the pristine PVDF membrane maintain the original structure (Figure 5- 
16a, b). Some E. coli cells in contact with antibacterial GOQDs become flattened or shrunken, while other bacteria in a binary fission process grow elongated.(221) In previous studies, the proposed graphene-based cytotoxicity mechanism mainly includes membrane stress induced by direct physical interaction and oxidative stress. $(224,225,237)$ In our case, we suggest that the highly-dispersed GOQDs with the ultra-small size and large specific surface area, similar to those of the graphene nanosheets reported by Akhavan et al(238) and Tu et al,(240) can perform a contactinhibition mode, which is direct insertion/cutting of lipid membranes and vigorous extraction of phospholipid molecules due to the strong dragging forces from graphene's unique structure of $\mathrm{sp}^{2}$ carbons, thus resulting in severe destruction of cell membrane and eventual loss of cytoplasm (Figure 5-16c,d, and 5-17).
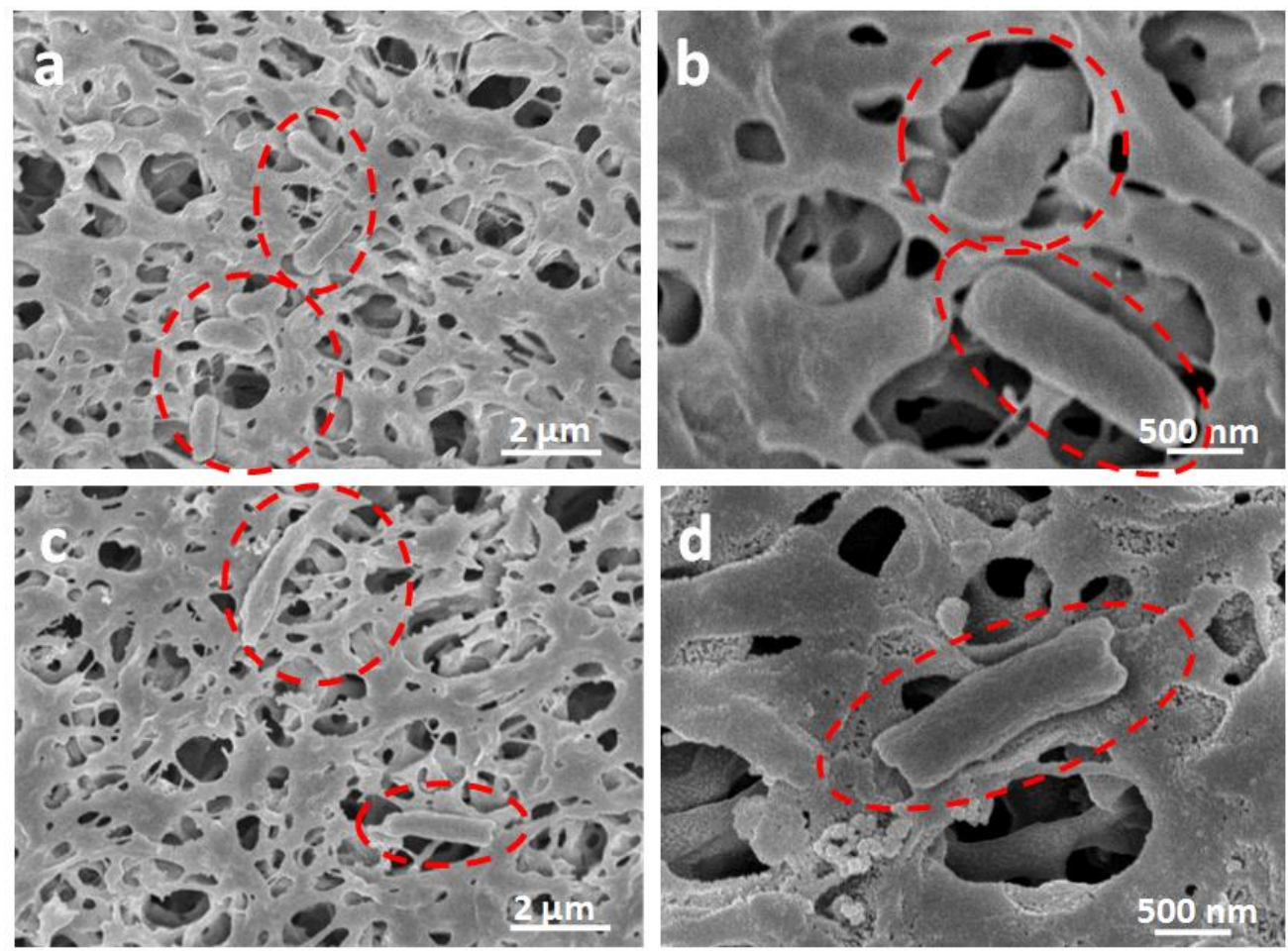

Figure 5-16 SEM images for normal E.coli cells at the surface of the pristine PVDF membrane (a, b) and GOQDs-PVDF membrane (c, d). 

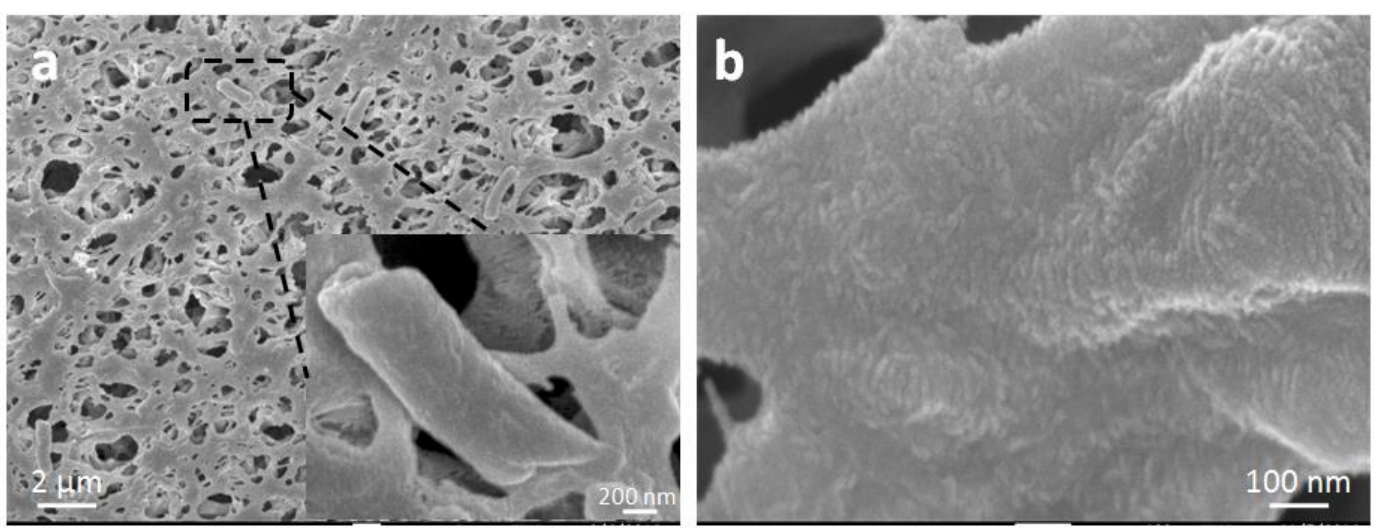

Figure 5-17. (a) SEM images of GOQDs-PVDF after $10 \mathrm{~h}$ filtration test with $E$. coli-containing feedwater; inset the $E$. coli cell on the membrane surface; (b) High-resolution SEM image of GOQDs-PVDF after $10 \mathrm{~h}$ water permision test with $E$. coli-containing feedwater.

The lipid extraction in the outer and inner membrane of bacterial cells (E. coli and $S$. aureus) can be induced by the strong dispersion interactions between GOQDs and lipid molecules. $(238,240)$ Even for bacterial cells in motion, as reported in the works involving polyacrylonitrile (PAN) hollow fiber decorated with $\mathrm{Ag}$ NPs/multiwalled carbon nanotube (Ag/MWNTs)(91) and the grafting of polyethyleneimine-coated Ag NPs on the surface of a plasma-treated polysulfone membrane, $(240,241)$ the immobilization of nanomaterial into appropriate matrixes can provider a promising prospect to improve a disinfection system with enhanced reliability and practical operation. Similarly, the GO nanosheets decorated membrane can also have bactericidal activity with a similar mechanism as described above on $E$. coli cells. However, due to their relatively tumid lateral size and wider size distribution, they could not fully cover the membrane surface with high dispersion (Figure 5-11). Some area without GO were exposed, enabling bacterial colonization and subsequently leading to the formation of cohesive biofilm structures. Therefore, the GOQDs-PVDF membrane is more effective in inhibiting the colonization of 
bacteria and the biofilm formation in comparison with pristine PVDF and GO-PVDF as depicted in Figure 5-18.

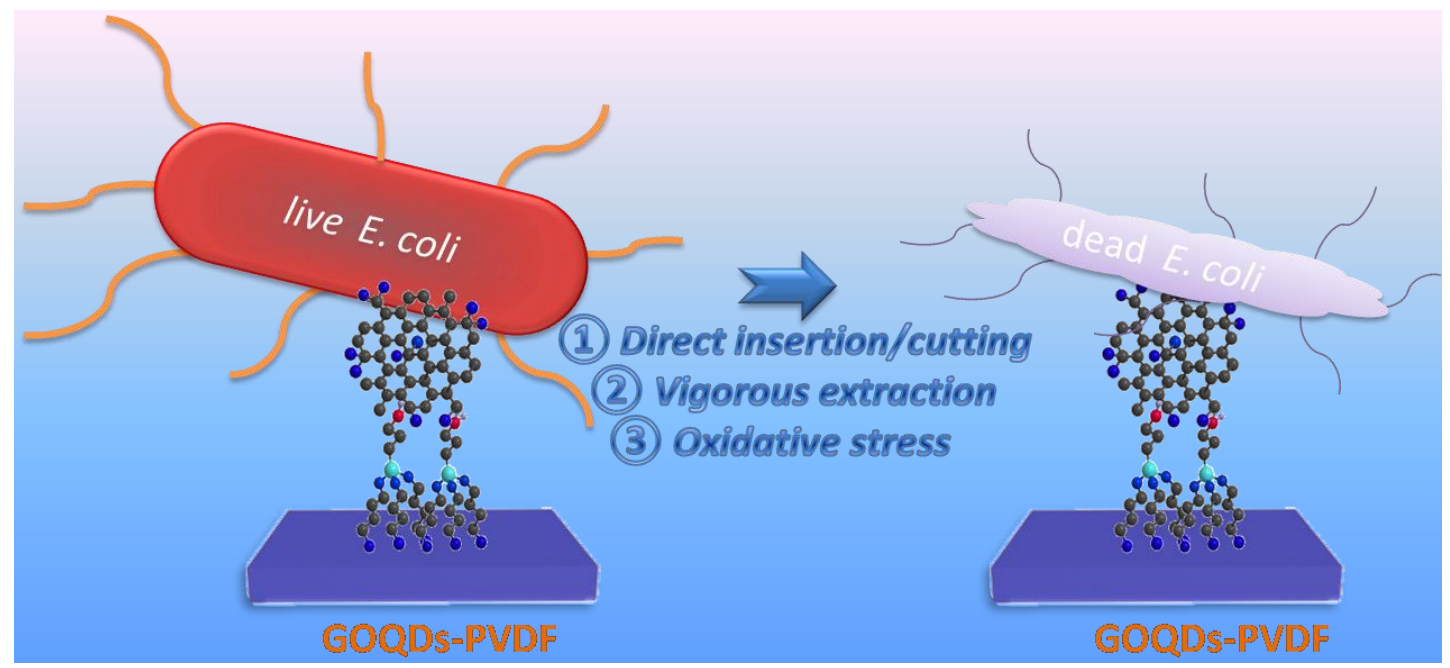

Figure 5-18. Schematic illustration for the bactericidal mechanism of GOQDsPVDF.

Oxidative stress is another way of inducing cell death, in which a specific vital cellular component or structure was oxidized or disrupted by graphene-based materials. $(221,225) \mathrm{GSH}$ oxidation in vitro can be used to evaluate oxidative stress induced by GO or GOQDs. GSH, a tripeptide molecule with thiol groups, can protect cellular components from the oxidative stress, is converted to glutathione disulfide when the thiol groups are oxidized to disulfide bond. The concentration of thiol groups of GHS can be quantified by the Ellman's assay, and the oxidative stress can be evaluated by observing the ratio of GSH loss. The oxidation of GSH was evaluated during the incubation with pristine PVDF, GO-PVDF, and GOQDs-PVDF. As shown in Figure 5-19, a noticeable fraction of GSH is oxidized after its exposure to GOQDs-PVDF $(63.3 \pm 2.42 \%)$ and GO-PVDF $(22.1 \pm 2.67 \%)$. Several studies have shown that GSH oxidation for single walled carbon nanotubes (SWNTs) and graphene-based materials is attributed to their excellent electronic conductivity.(224,225) When carbon nanomaterials are highly dispersed on the PVDF 
membrane surface, the facilitation of carbon nanomaterial networks are indicated as a unique combination of the electrons transport.(242) In our cases, we suggest that the difference of GSH oxidation extent between GOQDs-PVDF and GO-PVDF arises from the structure difference between 0 D GOQDs and 2 D GO. The homogeneous dispersion of smaller GOQDs on the same membrane surface, relative to GO sheets with lateral dimension, could lead to the exposure of a much larger fraction of active edges, $(241,243)$ facilitating the formation of a higher oxidative stress. Therefore, the bactericidal mechanism of GOQDs modified PVDF membrane evaluated with $E$. coli and $S$. aureus can be attributed to the direct contact interaction and oxidative stress introduced by GOQDs.

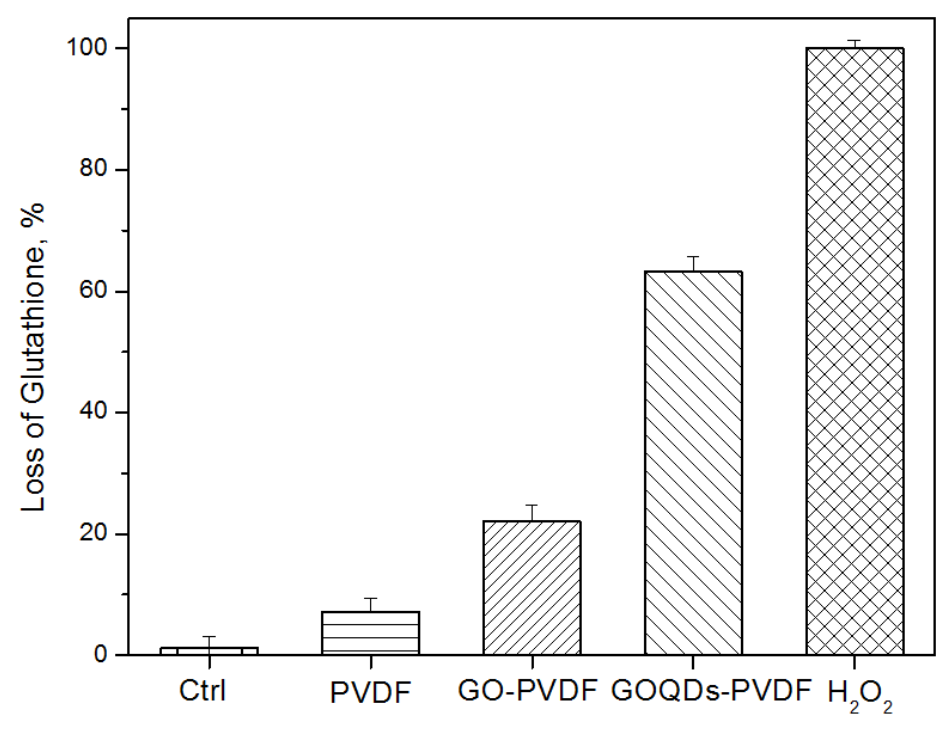

Figure 5-19 Loss of GSH (0.4 mM) after in vitro incubation with pristine PVDF and GO-PVDF for $1 \mathrm{~h}$. $\mathrm{H}_{2} \mathrm{O}_{2}(1 \mathrm{mM})$ is a positive control. The bicarbonate buffer ( $50 \mathrm{mM}$ at $\mathrm{pH} 8.6$ ) without membrane was used as a negative control.

\subsection{Summary}

The present study reported a first GOQDs covalent-functionalized PVDF membrane, and explored its use as an antimicrobial agent and preliminary as an antibiofouling membrane. The GOQDs-PVDF membrane shows much improved hydrophilic, antibacterial and antibiofouling properties without sacrificing the 
permeation property of the pristine PVDF membrane. A continuous water flux test using E. coli-containing solution indicated that the flux decrease over the GOQD modified PVDF was $24.3 \%$, which is remarkably lower than those over the bare PVDF $(88.4 \%)$ and over the GO-sheet modified PVDF $(65.7 \%)$ at 12 hours of filtration. Moreover, the GOQD coating layer effectively inhibits the growth of the bacterial cells and prevents the generation of the biofilm on the membrane surface, yielding a higher bacterial inactivation efficiency than those over 1 D SWNTs and 2 D GO modified membrane. The loading of GOQDs on the membrane could be easily tuned to achieve an optimal functionalized membrane combining excellent water permeation property, antimicrobial activity and biofouling resistance. The attractive features of hydrophilicity, stability, antibiofouling, and antibacterial properties of the GOQDs-PVDF membrane are promising in further enhancing membrane performance for the desalination/purification of water, and potentially separation of biomolecules. 


\section{Chapter 6 General conclusions and recommendations}

\section{for future research directions}

\subsection{General conclusions}

In this dissertation, multifarious catalysis activities and environmental applications of three different GQDs-based diverse-dimensional materials have been discussed in detail. GQDs combine the special optical and structural properties of quantum dots with the extraordinary electrical properties of $\mathrm{sp}^{2}$-carbon nanomaterials, large surface area and abundant functional groups on the edges, making them a highly unique nanomaterial as compared with conventional semiconductor QDs or other carbon-based nanomaterials such as carbon nanotubes and graphene. Throughout this $\mathrm{PhD}$ dissertation, nanostructured hybrid materials, with multifunctional properties have been successfully designed, synthesized and applied in various applications, including photoelectrochemical water splitting, thermal and electrochemical catalysis, and antibacterial performances.

The important contributions of this research work on the development of GQDsbased diverse-dimensional nanoarchitectures can be summarized as follows:

1. A facile, green, reproducible, and easily scalable and accessible LbL assembly strategy has been developed to fabricate spatially-ordered zero-dimensional $(\mathrm{M} / \mathrm{GQDs})_{\mathrm{n}}$ multilayer thin films. Their assembly is simply based on pronounced electrostatic interaction stemming from the intrinsically oppositely charged surfaces of metal NCs and GQDs. In addition, unique multilayered structural design enabled by LbL assembly offers tunable charge transfer pathways for (M/GQDs) $)_{\mathrm{n}}$ multilayer heterostructures. 
2. Multiple cooperative synergies of the nanodots building blocks manifested through versatile and superior catalytic performances of these well-defined $(\mathrm{M} / \mathrm{GQDs})_{\mathrm{n}}$ multilayer assemblies. The multifarious catalysis performances are including selective organic transformation of aromatic nitro compounds, electrocatalytic oxidation of methanol oxidation and light-irradiated PEC water splitting. These reactions were systematically studied and the corresponding catalytic mechanism in conjunction with the cooperative synergy of metal NCs and GQDs were elucidated. Stacked modes and interfacial structures of GQDs and metal NCs building blocks and, more significantly, catalytic performances of $(\mathrm{M} / \mathrm{GQDs})_{\mathrm{n}}$ multilayer can be finely tuned by simply altering the assembly cycle and sequence.

3. A simple synthetic approach has been developed in our work to prepare NGQDs under ambient conditions without involving any tedious synthetic procedures and harsh experiment conditions. This novel synthetic method would raise significant concern in the research communities who engage in the GQDs on the facile synthesis of non-metal elements doped GQDs.

4. Solar hydrogen production performances of well-defined N-GQDs/ZnO NWs heterostructures constructed via LbL assembly under both visible light and simulated solar light illumination were systematically probed and PEC mechanism of the N-GQDs/ZnO NWs heterostructures, especially the contributing role of N-GQDs during the PEC process, were specifically explored. This unique structural design afforded by LbL assembly offers tunable control over the decoration of N-GQDs on the 1D semiconductors and efficient charge transfer transport. 
5. GOQDs covalent-functionalized 2D membrane shows significantly improved hydrophilic, antibacterial and antibiofouling properties without sacrificing the permeation property of the pristine PVDF membrane. The loading of GOQDs on the membrane could be easily tuned to achieve an optimal functionalized membrane combining excellent water permeation property, antimicrobial activity and biofouling resistance.

6. The enhanced bactericidal activities and antifouling properties of GOQDsimmobilized PVDF membrane, superior to existing nano-carbon (such as 2D graphene oxide, 1D carbon nanotube) modified polymer membranes, has been demonstrated. A continuous flux test using bacterial-containing feed water indicated that the flux drop over the GOQD modified PVDF was $23 \%$, which is remarkably lower than those over the bare PVDF ( $86 \%$ ) and over the GO-sheet modified PVDF (62\%) at a 10-hour filtration period. Moreover, the GOQD coating layer effectively inhibits the growth of the bacterial cells and prevents the biofilm formation on the membrane surface, yielding a higher bacterial inactivation efficiency than those over $1 \mathrm{D}$ single-wall carbon nanotubes (SWNTs) and 2 D graphene oxide (GO) modified membrane.

7. Mechanistic investigation of the bactericidal activity of GOQDs-PVDF membrane reveals that the highly-dispersed GOQDs on the 2D membrane surface, with their ultra-small size and large specific surface area, can perform direct insertion/cutting of lipid membranes and vigorous extraction of phospholipid molecules of bacterial cells. The exposure of a larger fraction of active edges of GOQDs to facilitate the formation of a large 
oxidation stress, has also been explored to evaluate the distinctive bactericidal and antibiofouling performance of the current novel membrane.

\subsection{Recommendations for future research directions}

The main objective of this $\mathrm{PhD}$ program is to design a novel platform for rational construction of a large variety of GQDs-based 0D/0D, 0D/1D, 0D/2D functional materials at nanoscale as well as to investigate these materials for multifarious catalysis for advanced sustainable energy and environmental applications. Much progress on the development of GQDs-based materials including our GQDsbased multidimensional materials, has been made in the past several years. However, further endeavors on these hybrid functional materials are expected to be integrated widely into industry and to achieve breakthrough performance for our real-life applications in the future. Many challenges are waiting to be overcome, such as expensive synthesis cost, extreme operating environment, poor system stability, and low efficiency.

From our side, the recommendations for future research directions are suggested as following:

1. The morphologies, sizes, structures, and the properties of GQDs cannot be precisely controlled during the variety of fabrication methods reported in the literature in Chapter 2. Bottom-up approaches, including dehydration or assembly of small molecules, produce GQDs with large size distribution, which particularly are not favorable in photocatalytic research. Top-down routes, such as electrochemical etching, can be applied to fabricate sizecontrolled GQDs from graphene or CNTs. However, low yield and the harsh reaction conditions of this top-down route hinder the promotion of the applications of the synthesized well-defined GQDs. Hence, synthesis 
strategies with large scale and low cost towards GQDs with narrow size distribution and highly-enhanced performance are greatly expected.

2. The hybridization between GQDs and other $0 \mathrm{D} / 1 \mathrm{D} / 2 \mathrm{D} / 3 \mathrm{D}$ materials plays an important role for the interfacial charge transfer. Generally, GQDs are physically connected with the other materials by mixing routes in the most papers. Effective chemical bonding between the two components can be more stable, improving the electron-transfer process. It is worthwhile to develop simple chemical linkage strategies involving functionalized linking groups on GQDs-based nanostructures and the subsequent effect of the chemical bond can be investigated widely in catalysis and environmental performance. Moreover, given the unique properties of GQDs, I believe more up and coming GQDs-based nanocomposite will be exploited in solar-energy conversion and environmental issues (such as, $\mathrm{CO}_{2}$ reduction catalysis, water purification et al.).

3. It is advisable for scientist communities to further develop LbL assembly technologies involving various unique components to fabricate diverse multifunctional thin films and nanobuilding block with excellent physicochemical properties. The graphene-generated "gold-rush" period unleashes the extraordinary properties of graphene in recent several years, which is capable to be further utilized as a unique building block for selfassembly of $2 \mathrm{D}$ or $3 \mathrm{D}$ architectures. Alternatively, apart from graphene and graphene quantum dots, various inorganic nanosheets and quantum dots such as transition metal dichalcogenide (TMD) nanosheets and nanocrystals have attracted tremendous interest in the past few years due to their excellent electronic structures and physicochemical properties. These nanosheets and 
nanocrystals can be utilized as constructing components for self-assembly of a large variety of multi-functional and multi-dimensional nanomaterials. 


\section{Reference}

[1] Kroto, H. W., Heath, J. R., O'Brien, S. C., Curl, R. F., \& Smalley, R. E. (1985). C60: buckminsterfullerene. Nature, 318(318), 162.

[2] Iijima, S. (1991). Helical Microtubules of Graphitic Carbon. Nature, 354(6348), 56-58.

[3] Novoselov, K. S., Geim, A. K., Morozov, S. V., Jiang, D., Zhang, Y., Dubonos, S. V., Grigorieva, I. V., \& Firsov, A. A. (2004). Electric field effect in atomically thin carbon films. Science, 306(5696), 666-669.

[4] Brody, H. (2012). Graphene. Nature, 483(7389), S29-S29.

[5] Geim, A. K., \& MacDonald, A. H. (2007). Graphene: Exploring carbon flatland. Physics Today, 60(8), 35-41.

[6] Long, R., Casanova, D., Fang, W. H., \& Prezhdo, O. V. (2017). DonorAcceptor Interaction Determines the Mechanism of Photoinduced Electron Injection from Graphene Quantum Dots into TiO2: pi-Stacking Supersedes Covalent Bonding. Journal of the American Chemical Society, 139(7), 2619-2629.

[7] Tetsuka, H., Nagoya, A., Fukusumi, T., \& Matsui, T. (2016). Molecularly Designed, Nitrogen-Functionalized Graphene Quantum Dots for Optoelectronic Devices. Advanced Materials, 28(23), 4632-4638.

[8] Suzuki, N., Wang, Y., Elvati, P., Qu, Z. B., Kim, K., Jiang, S., Baumeister, E., Lee, J., Yeom, B., Bahng, J. H., Lee, J., Violi, A., \& Kotov, N. A. (2016). Chiral Graphene Quantum Dots. ACS Nano, 10(2), 1744-1755.

[9] Li, N., Than, A., Sun, C., Tian, J., Chen, J., Pu, K., Dong, X., \& Chen, P. (2016). Monitoring Dynamic Cellular Redox Homeostasis Using FluorescenceSwitchable Graphene Quantum Dots. ACS Nano, 10(12), 11475-11482.

[10] Lee, J., Wong, D., Velasco Jr, J., Rodriguez-Nieva, J. F., Kahn, S., Tsai, H.-Z., Taniguchi, T., Watanabe, K., Zettl, A., Wang, F., Levitov, L. S., \& Crommie, M. F. (2016). Imaging electrostatically confined Dirac fermions in graphene quantum dots. Nature Physics, 12(11), 1032-1036.

[11] Gutiérrez, C., Brown, L., Kim, C.-J., Park, J., \& Pasupathy, A. N. (2016). Klein tunnelling and electron trapping in nanometre-scale graphene quantum dots. Nature Physics, 12(11), 1069-1075.

[12] Freitag, N. M., Chizhova, L. A., Nemes-Incze, P., Woods, C. R., Gorbachev, R. V., Cao, Y., Geim, A. K., Novoselov, K. S., Burgdorfer, J., Libisch, F., \& Morgenstern, M. (2016). Electrostatically Confined Monolayer Graphene Quantum Dots with Orbital and Valley Splittings. Nano Lettersers, 16(9), 5798-5805.

[13] El Fatimy, A., Myers-Ward, R. L., Boyd, A. K., Daniels, K. M., Gaskill, D. K., \& Barbara, P. (2016). Epitaxial graphene quantum dots for high-performance terahertz bolometers. Nature Nanotechnology, 11(4), 335-338.

[14] Ponomarenko, L. A., Schedin, F., Katsnelson, M. I., Yang, R., Hill, E. W., Novoselov, K. S., \& Geim, A. K. (2008). Chaotic dirac billiard in graphene quantum dots. Science, 320(5874), 356-358.

[15] Lu, J., Yeo, P. S. E., Gan, C. K., Wu, P., \& Loh, K. P. (2011). Transforming C-60 molecules into graphene quantum dots. Nature Nanotechnology, 6(4), 247-252.

[16] Yeh, T. F., Teng, C. Y., Chen, S. J., \& Teng, H. S. (2014). Nitrogen-Doped Graphene Oxide Quantum Dots as Photocatalysts for Overall Water-Splitting under Visible Light Illumination. Advanced Materials, 26(20), 3297-3303. 
[17] Cheng, H. H., Zhao, Y., Fan, Y. Q., Xie, X. J., Qu, L. T., \& Shi, G. Q. (2012). Graphene-Quantum-Dot Assembled Nanotubes: A New Platform for Efficient Raman Enhancement. ACS Nano, 6(3), 2237-2244.

[18] Zhang, Z. P., Zhang, J., Chen, N., \& Qu, L. T. (2012). Graphene quantum dots: an emerging material for energy-related applications and beyond. Energy \& Environmental Science, 5(10), 8869-8890.

[19] Peng, J., Gao, W., Gupta, B. K., Liu, Z., Romero-Aburto, R., Ge, L. H., Song, L., Alemany, L. B., Zhan, X. B., Gao, G. H., Vithayathil, S. A., Kaipparettu, B. A., Marti, A. A., Hayashi, T., Zhu, J. J., \& Ajayan, P. M. (2012). Graphene Quantum Dots Derived from Carbon Fibers. Nano Lettersers, 12(2), 844-849.

[20] Liu, R. L., Wu, D. Q., Feng, X. L., \& Mullen, K. (2011). Bottom-Up Fabrication of Photoluminescent Graphene Quantum Dots with Uniform Morphology. Journal of the American Chemical Society, 133(39), 15221-15223.

[21] Yan, X., Cui, X., Li, B. S., \& Li, L. S. (2010). Large, Solution-Processable Graphene Quantum Dots as Light Absorbers for Photovoltaics. Nano Letters, 10(5), 1869-1873.

[22] Zhang, N., Yang, M. Q., Liu, S. Q., Sun, Y. G., \& Xu, Y. J. (2015). Waltzing with the Versatile Platform of Graphene to Synthesize Composite Photocatalysts. Chemical Reviews, 115(18), 10307-10377.

[23] Pan, D., Zhang, J., Li, Z., \& Wu, M. (2010). Hydrothermal route for cutting graphene sheets into blue-luminescent graphene quantum dots. Advanced Materials, 22(6), 734-738.

[24] Zhu, S. J., Zhang, J. H., Tang, S. J., Qiao, C. Y., Wang, L., Wang, H. Y., Liu, X., Li, B., Li, Y. F., Yu, W. L., Wang, X. F., Sun, H. C., \& Yang, B. (2012). Surface Chemistry Routes to Modulate the Photoluminescence of Graphene Quantum Dots: From Fluorescence Mechanism to Up-Conversion Bioimaging Applications. Advanced Functional Materials, 22(22), 4732-4740.

[25] Zhu, S. J., Zhang, J. H., Qiao, C. Y., Tang, S. J., Li, Y. F., Yuan, W. J., Li, B., Tian, L., Liu, F., Hu, R., Gao, H. N., Wei, H. T., Zhang, H., Sun, H. C., \& Yang, B. (2011). Strongly green-photoluminescent graphene quantum dots for bioimaging applications. Chemical Communications, 47(24), 6858-6860.

[26] Dong, Y., Chen, C., Zheng, X., Gao, L., Cui, Z., Yang, H., Guo, C., Chi, Y., \& Li, C. M. (2012). One-step and high yield simultaneous preparation of single- and multi-layer graphene quantum dots from CX-72 carbon black. Journal of Materials Chemistry, 22(18), 8764.

[27] Razmi, H., \& Mohammad-Rezaei, R. (2013). Graphene quantum dots as a new substrate for immobilization and direct electrochemistry of glucose oxidase: Application to sensitive glucose determination. Biosensors \& Bioelectronics, 41, 498504.

[28] Zhao, H. M., Chang, Y. Y., Liu, M., Gao, S., Yu, H. T., \& Quan, X. (2013). A universal immunosensing strategy based on regulation of the interaction between graphene and graphene quantum dots. Chemical Communications, 49(3), 234-236.

[29] Gupta, V., Chaudhary, N., Srivastava, R., Sharma, G. D., Bhardwaj, R., \& Chand, S. (2011). Luminscent Graphene Quantum Dots for Organic Photovoltaic Devices. Journal of the American Chemical Society, 133(26), 9960-9963.

[30] Zhuo, S. J., Shao, M. W., \& Lee, S. T. (2012). Upconversion and Downconversion Fluorescent Graphene Quantum Dots: Ultrasonic Preparation and Photocatalysis. ACS Nano, 6(2), 1059-1064.

[31] Tang, L. B., Ji, R. B., Cao, X. K., Lin, J. Y., Jiang, H. X., Li, X. M., Teng, K. S., Luk, C. M., Zeng, S. J., Hao, J. H., \& Lau, S. P. (2012). Deep Ultraviolet 
Photoluminescence of Water-Soluble Self-Passivated Graphene Quantum Dots. ACS Nano, 6(6), 5102-5110.

[32] Zhou, X. J., Zhang, Y., Wang, C., Wu, X. C., Yang, Y. Q., Zheng, B., Wu, H. X., Guo, S. W., \& Zhang, J. Y. (2012). Photo-Fenton Reaction of Graphene Oxide: A New Strategy to Prepare Graphene Quantum Dots for DNA Cleavage. ACS Nano, 6(8), 6592-6599.

[33] Zhang, Z., Zhang, J., Chen, N., \& Qu, L. (2012). Graphene quantum dots: an emerging material for energy-related applications and beyond. Energy \& Environmental Science, 5(10), 8869.

[34] Kim, J. K., Park, M. J., Kim, S. J., Wang, D. H., Cho, S. P., Bae, S., Park, J. H., \& Hong, B. H. (2013). Balancing Light Absorptivity and Carrier Conductivity of Graphene Quantum Dots for High-Efficiency Bulk Heterojunction Solar Cells. ACS Nano, 7(8), 7207-7212.

[35] Li, Y., Hu, Y., Zhao, Y., Shi, G. Q., Deng, L. E., Hou, Y. B., \& Qu, L. T. (2011). An Electrochemical Avenue to Green-Luminescent Graphene Quantum Dots as Potential Electron-Acceptors for Photovoltaics. Advanced Materials, 23(6), 776781.

[36] Zhu, Z. L., Ma, J. A., Wang, Z. L., Mu, C., Fan, Z. T., Du, L. L., Bai, Y., Fan, L. Z., Yan, H., Phillips, D. L., \& Yang, S. H. (2014). Efficiency Enhancement of Perovskite Solar Cells through Fast Electron Extraction: The Role of Graphene Quantum Dots. Journal of the American Chemical Society, 136(10), 3760-3763.

[37] Chao, D. L., Zhu, C. R., Xia, X. H., Liu, J. L., Zhang, X., Wang, J., Liang, P., Lin, J. Y., Zhang, H., Shen, Z. X., \& Fan, H. J. (2015). Graphene Quantum Dots Coated $\mathrm{VO}_{2}$ Arrays for Highly Durable Electrodes for Li and $\mathrm{Na}$ Ion Batteries. Nano Letters, 15(1), 565-573.

[38] Zhu, C. R., Chao, D. L., Sun, J., Bacho, I. M., Fan, Z. X., Ng, C. F., Xia, X. H., Huang, H., Zhang, H., Shen, Z. X., Ding, G. Q., \& Fan, H. J. (2015). Enhanced Lithium Storage Performance of $\mathrm{CuO}$ Nanowires by Coating of Graphene Quantum Dots. Advanced Materials Interfaces, 2(2).

[39] Liu, W. W., Feng, Y. Q., Yan, X. B., Chen, J. T., \& Xue, Q. J. (2013). Superior Micro-Supercapacitors Based on Graphene Quantum Dots. Advanced Functional Materials, 23(33), 4111-4122.

[40] Mondal, S., Rana, U., \& Malik, S. (2015). Graphene quantum dot-doped polyaniline nanofiber as high performance supercapacitor electrode materials. Chemical Communications, 51(62), 12365-12368.

[41] Wang, Z. F., Zeng, H. D., \& Sun, L. Y. (2015). Graphene quantum dots: versatile photoluminescence for energy, biomedical, and environmental applications. Journal of Materials Chemistry C, 3(6), 1157-1165.

[42] Lu, Q., Zhang, Y. J., \& Liu, S. Q. (2015). Graphene quantum dots enhanced photocatalytic activity of zinc porphyrin toward the degradation of methylene blue under visible-light irradiation. Journal of Materials Chemistry A, 3(16), 8552-8558.

[43] Pan, D. Y., Jiao, J. K., Li, Z., Guo, Y. T., Feng, C. Q., Liu, Y., Wang, L., \& Wu, M. H. (2015). Efficient Separation of Electron-Hole Pairs in Graphene Quantum Dots by TiO2 Heterojunctions for Dye Degradation. ACS Sustainable Chemistry \& Engineering, 3(10), 2405-2413.

[44] Ristic, B. Z., Milenkovic, M. M., Dakic, I. R., Todorovic-Markovic, B. M., Milosavljevic, M. S., Budimir, M. D., Paunovic, V. G., Dramicanin, M. D., Markovic, Z. M., \& Trajkovic, V. S. (2014). Photodynamic antibacterial effect of graphene quantum dots. Biomaterials, 35(15), 4428-4435. 
[45] Habiba, K., Encarnacion-Rosado, J., Garcia-Pabon, K., Villalobos-Santos, J. C., Makarov, V. I., Avalos, J. A., Weiner, B. R., \& Morell, G. (2016). Improving cytotoxicity against cancer cells by chemo-photodynamic combined modalities using silver-graphene quantum dots nanocomposites. International Journal of Nanomedicine, 11, 107-119

[46] Dong, Y. Q., Li, G. L., Zhou, N. N., Wang, R. X., Chi, Y. W., \& Chen, G. N. (2012). Graphene Quantum Dot as a Green and Facile Sensor for Free Chlorine in Drinking Water. Analytical Chemistry, 84(19), 8378-8382.

[47] Zhang, C., Wei, K., Zhang, W., Bai, Y., Sun, Y., \& Gu, J. (2017). Graphene Oxide Quantum Dots Incorporated into a Thin Film Nanocomposite Membrane with High Flux and Antifouling Properties for Low-Pressure Nanofiltration. ACS Applied Materials \& Interfaces, 9(12), 11082-11094.

[48] Zeng, Z., Yu, D., He, Z., Liu, J., Xiao, F. X., Zhang, Y., Wang, R., Bhattacharyya, D., \& Tan, T. T. (2016). Graphene Oxide Quantum Dots Covalently Functionalized PVDF Membrane with Significantly-Enhanced Bactericidal and Antibiofouling Performances. Scientific Reports, 6, 20142.

[49] Choi, Y., Gu, M., Park, J., Song, H. K., \& Kim, B. S. (2012). Graphene Multilayer Supported Gold Nanoparticles for Efficient Electrocatalysts Toward Methanol Oxidation. Advanced Energy Materials, 2(12), 1510-1518.

[50] Gui, J. H., Pan, C. M., Jin, Y., Qin, T., Lo, J. C., Lee, B. J., Spergel, S. H., Mertzman, M. E., Pitts, W. J., La Cruz, T. E., Schmidt, M. A., Darvatkar, N., Natarajan, S. R., \& Baran, P. S. (2015). Practical olefin hydroamination with nitroarenes. Science, 348(6237), 886-891.

[51] Jagadeesh, R. V., Surkus, A. E., Junge, H., Pohl, M. M., Radnik, J., Rabeah, J., Huan, H. M., Schunemann, V., Bruckner, A., \& Beller, M. (2013). Nanoscale Fe2O3Based Catalysts for Selective Hydrogenation of Nitroarenes to Anilines. Science, 342(6162), 1073-1076.

[52] Jagadeesh, R. V., Natte, K., Junge, H., \& Beller, M. (2015). Nitrogen-Doped Graphene-Activated Iron-Oxide-Based Nanocatalysts for Selective Transfer Hydrogenation of Nitroarenes. ACS Catalysis, 5(3), 1526-1529.

[53] Guancai Xie, K. Z., Beidou Guo, Qian Liu, Liang Fang, Jian Ru Gong. (2013). Graphene-Based Materials for Hydrogen Generation from Light-Driven Water Splitting. Advanced Materials, 25(28), 3820-3839.

[54] Choi, Y., Bae, H. S., Seo, E., Jang, S., Park, K. H., \& Kim, B. S. (2011). Hybrid gold nanoparticle-reduced graphene oxide nanosheets as active catalysts for highly efficient reduction of nitroarenes. Journal of Materials Chemistry, 21(39), 15431-15436.

[55] Adhikari, B., Biswas, A., \& Banerjee, A. (2012). Graphene Oxide-Based Hydrogels to Make Metal Nanoparticle-Containing Reduced Graphene Oxide-Based Functional Hybrid Hydrogels. ACS Applied Materials \& Interfaces, 4(10), 5472-5482. [56] Yu, H. J., Shi, R., Zhao, Y. F., Waterhouse, G. I. N., Wu, L. Z., Tung, C. H., \& Zhang, T. R. (2016). Smart Utilization of Carbon Dots in Semiconductor Photocatalysis. Advanced Materials, 28(43), 9454-9477.

[57] Zhou, M., Zeng, C. J., Chen, Y. X., Zhao, S., Sfeir, M. Y., Zhu, M. Z., \& Jin, R. C. (2016). Evolution from the plasmon to exciton state in ligand-protected atomically precise gold nanoparticles. Nature Communications, 7, 13240.

[58] Crudden, C. M., Horton, J. H., Narouz, M. R., Li, Z. J., Smith, C. A., Munro, K., Baddeley, C. J., Larrea, C. R., Drevniok, B., Thanabalasingam, B., McLean, A. B., Zenkina, O. V., Ebralidze, I. I., She, Z., Kraatz, H. B., Mosey, N. J., Saunders, L. N., $\&$ Yagi, A. (2016). Simple direct formation of self-assembled N-heterocyclic carbene 
monolayers on gold and their application in biosensing. Nature Communications, 7 , 12654.

[59] Salorinne, K., Man, R. W. Y., Li, C.-H., Taki, M., Nambo, M., \& Crudden, C. M. (2017). Water-Soluble N-Heterocyclic Carbene-Protected Gold Nanoparticles: Size-Controlled Synthesis, Stability, and Optical Properties. Angewandte Chemie International Edition, 56(22), 6198-6202.

[60] Jin, R. C., Zeng, C. J., Zhou, M., \& Chen, Y. X. (2016). Atomically Precise Colloidal Metal Nanoclusters and Nanoparticles: Fundamentals and Opportunities. Chemical Reviews, 116(18), 10346-10413.

[61] Wu, X. C., Guo, S. W., \& Zhang, J. Y. (2015). Selective oxidation of veratryl alcohol with composites of $\mathrm{Au}$ nanoparticles and graphene quantum dots as catalysts. Chemical Communications, 51(29), 6318-6321.

[62] Yin, H. J., Tang, H. J., Wang, D., Gao, Y., \& Tang, Z. Y. (2012). Facile Synthesis of Surfactant-Free Au Cluster/Graphene Hybrids for High-Performance Oxygen Reduction Reaction. ACS Nano, 6(9), 8288-8297.

[63] Luo, P. H., Jiang, L. Q., Zhang, W. L., \& Guan, X. F. (2015). Graphene quantum dots/Au hybrid nanoparticles as electrocatalyst for hydrogen evolution reaction. Chemical Physics Letters, 641, 29-32.

[64] Zhang, Y., Wu, C. Y., Zhou, X. J., Wu, X. C., Yang, Y. Q., Wu, H. X., Guo, S. W., \& Zhang, J. Y. (2013). Graphene quantum dots/gold electrode and its application in living cell $\mathrm{H} 2 \mathrm{O} 2$ detection. Nanoscale, 5(5), 1816-1819.

[65] Ran, X., Sun, H. J., Pu, F., Ren, J. S., \& Qu, X. G. (2013). Ag Nanoparticledecorated graphene quantum dots for label-free, rapid and sensitive detection of $\mathrm{Ag}^{+}$ and biothiols. Chemical Communications, 49(11), 1079-1081.

[66] Wang, S., Zhao, Q. F., Wei, H. M., Wang, J. Q., Cho, M. Y., Cho, H. S., Terasaki, O., \& Wan, Y. (2013). Aggregation-Free Gold Nanoparticles in Ordered Mesoporous Carbons: Toward Highly Active and Stable Heterogeneous Catalysts. Journal of the American Chemical Society, 135(32), 11849-11860.

[67] Yan, H., Cheng, H., Yi, H., Lin, Y., Yao, T., Wang, C. L., Li, J. J., Wei, S. Q., \& Lu, J. L. (2015). Single-Atom Pd-1/Graphene Catalyst Achieved by Atomic Layer Deposition: Remarkable Performance in Selective Hydrogenation of 1,3-Butadiene. Journal of the American Chemical Society, 137(33), 10484-10487.

[68] Albanese, A., \& Chan, W. C. W. (2011). Effect of Gold Nanoparticle Aggregation on Cell Uptake and Toxicity. ACS Nano, 5(7), 5478-5489.

[69] Yang, H. B., Miao, J. W., Hung, S. F., Huo, F. W., Chen, H. M., \& Liu, B. (2014). Stable Quantum Dot Photoelectrolysis Cell for Unassisted Visible Light Solar Water Splitting. ACS Nano, 8(10), 10403-10413.

[70] Li, Z. S., Luo, W. J., Zhang, M. L., Feng, J. Y., \& Zou, Z. G. (2013). Photoelectrochemical cells for solar hydrogen production: current state of promising photoelectrodes, methods to improve their properties, and outlook. Energy \& Environmental Science, 6(2), 347-370.

[71] Walter, M. G., Warren, E. L., McKone, J. R., Boettcher, S. W., Mi, Q. X., Santori, E. A., \& Lewis, N. S. (2010). Solar Water Splitting Cells. Chemical Reviews, $110(11), 6446-6473$.

[72] Lewis, N. S., \& Nocera, D. G. (2006). Powering the planet: Chemical challenges in solar energy utilization. Proceedings of the National Academy of Sciences, 103(43), 15729-15735.

[73] Ng, Y. H., Ikeda, S., Matsumura, M., \& Amal, R. (2012). A perspective on fabricating carbon-based nanomaterials by photocatalysis and their applications. Energy \& Environmental Science, 5(11), 9307-9318. 
[74] Iwashina, K., Iwase, A., Ng, Y. H., Amal, R., \& Kudo, A. (2015). ZSchematic Water Splitting into H-2 and O-2 Using Metal Sulfide as a HydrogenEvolving Photocatalyst and Reduced Graphene Oxide as a Solid-State Electron Mediator. Journal of the American Chemical Society, 137(2), 604-607.

[75] Fujishima, A., \& Honda, K. (1972). Electrochemical Photolysis of Water at a Semiconductor Electrode. Nature, 238(5358), 37.

[76] Qiu, Y. C., Yan, K. Y., Deng, H., \& Yang, S. H. (2012). Secondary Branching and Nitrogen Doping of $\mathrm{ZnO}$ Nanotetrapods: Building a Highly Active Network for Photoelectrochemical Water Splitting. Nano Lettersers, 12(1), 407-413.

[77] Lin, Y. J., Xu, Y., Mayer, M. T., Simpson, Z. I., McMahon, G., Zhou, S., \& Wang, D. W. (2012). Growth of p-Type Hematite by Atomic Layer Deposition and Its Utilization for Improved Solar Water Splitting. Journal of the American Chemical Society, 134(12), 5508-5511.

[78] Liu, S. Q., Tang, Z. R., Sun, Y. G., Colmenares, J. C., \& Xu, Y. J. (2015). One-dimension-based spatially ordered architectures for solar energy conversion. Chemical Society Reviews, 44(15), 5053-5075.

[79] Xiao, F. X., Miao, J. W., Tao, H. B., Hung, S. F., Wang, H. Y., Yang, H. B., Chen, J. Z., Chen, R., \& Liu, B. (2015). One-Dimensional Hybrid Nanostructures for Heterogeneous Photocatalysis and Photoelectrocatalysis. Small, 11(18), 2115-2131.

[80] Chen, H. M., Chen, C. K., Chang, Y. C., Tsai, C. W., Liu, R. S., Hu, S. F., Chang, W. S., \& Chen, K. H. (2010). Quantum Dot Monolayer Sensitized ZnO Nanowire-Array Photoelectrodes: True Efficiency for Water Splitting. Angewandte Chemie-International Edition, 49(34), 5966-5969.

[81] Xiao, F. X., Hung, S. F., Tao, H. B., Miao, J. W., Yang, H. B., \& Liu, B. (2014). Spatially branched hierarchical $\mathrm{ZnO}$ nanorod-TiO2 nanotube array heterostructures for versatile photocatalytic and photoelectrocatalytic applications: towards intimate integration of 1D-1D hybrid nanostructures. Nanoscale, 6(24), 14950-14961.

[82] Wang, G. M., Wang, H. Y., Ling, Y. C., Tang, Y. C., Yang, X. Y., Fitzmorris, R. C., Wang, C. C., Zhang, J. Z., \& Li, Y. (2011). Hydrogen-Treated TiO2 Nanowire Arrays for Photoelectrochemical Water Splitting. Nano Lettersers, 11(7), 3026-3033.

[83] Decher, G. (1997). Fuzzy nanoassemblies: Toward layered polymeric multicomposites. Science, 277(5330), 1232-1237.

[84] Xiao, F. X., Zeng, Z. P., \& Liu, B. (2015). Bridging the Gap: Electron Relay and Plasmonic Sensitization of Metal Nanocrystals for Metal Clusters. Journal of the American Chemical Society, 137(33), 10735-10744.

[85] Xiao, F. X., Miao, J. W., \& Liu, B. (2014). Layer-by-Layer Self-Assembly of CdS Quantum Dots/Graphene Nanosheets Hybrid Films for Photoelectrochemical and Photocatalytic Applications. Journal of the American Chemical Society, 136(4), 1559-1569.

[86] Tang, Q. W., Wu, J. H., Li, Q. H., \& Lin, J. M. (2008). High conducting multilayer films from poly(sodium styrenesulfonate) and graphite nanoplatelets by layer-by-layer self-assembly. Polymer, 49(24), 5329-5335.

[87] Shannon, M. A., Bohn, P. W., Elimelech, M., Georgiadis, J. G., Marinas, B. J., \& Mayes, A. M. (2008). Science and technology for water purification in the coming decades. Nature, 452(7185), 301-310.

[88] Garcia-Garcia, G., Jha, V., \& Comm, W. K. D. S. (2015). NEPHROLOGY IN THE DEVELOPING WORLD Chronic kidney disease in disadvantaged populations. Nature Reviews Nephrology, 11(3), 127-128. 
[89] Sima, L. C., \& Elimelech, M. (2013). More than a Drop in the Bucket: Decentralized Membrane-Based Drinking Water Refill Stations in Southeast Asia. Environmental Science \& Technology, 47(14), 7580-7588.

[90] Pendergast, M. M., \& Hoek, E. M. V. (2011). A review of water treatment membrane nanotechnologies. Energy \& Environmental Science, 4(6), 1946-1971.

[91] Gunawan, P., Guan, C., Song, X. H., Zhang, Q. Y., Leong, S. S. J., Tang, C. Y., Chen, Y., Chan-Park, M. B., Chang, M. W., Wang, K. A., \& Xu, R. (2011). Hollow Fiber Membrane Decorated with Ag/MWNTs: Toward Effective Water Disinfection and Biofouling Control. ACS Nano, 5(12), 10033-10040.

[92] Sun, H. J., Gao, N., Dong, K., Ren, J. S., \& Qu, X. G. (2014). Graphene Quantum Dots-Band-Aids Used for Wound Disinfection. ACS Nano, 8(6), 6202-6210. [93] Kang, G. D., \& Cao, Y. M. (2014). Application and modification of poly(vinylidene fluoride) (PVDF) membranes - A review. Journal of Membrane Science, 463, 145-165.

[94] Shi, H. Y., Liu, F., \& Xue, L. X. (2013). Fabrication and characterization of antibacterial PVDF hollow fibre membrane by doping Ag-loaded zeolites. Journal of Membrane Science, 437, 205-215.

[95] Liu, R., Wu, D., Feng, X., \& Mullen, K. (2011). Bottom-up fabrication of photoluminescent graphene quantum dots with uniform morphology. Journal of the American Chemical Society, 133(39), 15221-15223.

[96] Yan, X., Cui, X., \& Li, L. S. (2010). Synthesis of Large, Stable Colloidal Graphene Quantum Dots with Tunable Size. Journal of the American Chemical Society, 132(17), 5944-5945.

[97] Yan, X., Cui, X., Li, B., \& Li, L. S. (2010). Large, solution-processable graphene quantum dots as light absorbers for photovoltaics. Nano Letters, 10(5), 1869-1873.

[98] Li, Q., Zhang, S., Dai, L., \& Li, L. S. (2012). Nitrogen-doped colloidal graphene quantum dots and their size-dependent electrocatalytic activity for the oxygen reduction reaction. Journal of the American Chemical Society, 134(46), 18932-18935.

[99] Lu, J., Yeo, P. S., Gan, C. K., Wu, P., \& Loh, K. P. (2011). Transforming C60 molecules into graphene quantum dots. Nature Nanotechnology, 6(4), 247-252.

[100] Wang, L., Wang, Y., Xu, T., Liao, H., Yao, C., Liu, Y., Li, Z., Chen, Z., Pan, D., Sun, L., \& Wu, M. (2014). Gram-scale synthesis of single-crystalline graphene quantum dots with superior optical properties. Nature Communications, 5, 5357.

[101] Li, X., Lau, S. P., Tang, L., Ji, R., \& Yang, P. (2014). Sulphur doping: a facile approach to tune the electronic structure and optical properties of graphene quantum dots. Nanoscale, 6(10), 5323-5328.

[102] Qu, D., Sun, Z., Zheng, M., Li, J., Zhang, Y., Zhang, G., Zhao, H., Liu, X., \& Xie, Z. (2015). Three Colors Emission from S,N Co-doped Graphene Quantum Dots for Visible Light H2Production and Bioimaging. Advanced Optical Materials, 3(3), 360-367.

[103] Kosynkin, D. V., Higginbotham, A. L., Sinitskii, A., Lomeda, J. R., Dimiev, A., Price, B. K., \& Tour, J. M. (2009). Longitudinal unzipping of carbon nanotubes to form graphene nanoribbons. Nature, 458(7240), 872-876.

[104] Pan, D., Guo, L., Zhang, J., Xi, C., Xue, Q., Huang, H., Li, J., Zhang, Z., Yu, W., Chen, Z., Li, Z., \& Wu, M. (2012). Cutting sp2 clusters in graphene sheets into colloidal graphene quantum dots with strong green fluorescence. Journal of Materials Chemistry, 22(8), 3314. 
[105] Shen, J. H., Zhu, Y. H., Chen, C., Yang, X. L., \& Li, C. Z. (2011). Facile preparation and upconversion luminescence of graphene quantum dots. Chemical Communications, 47(9), 2580-2582.

[106] Shen, J. H., Zhu, Y. H., Yang, X. L., Zong, J., Zhang, J. M., \& Li, C. Z. (2012). One-pot hydrothermal synthesis of graphene quantum dots surface-passivated by polyethylene glycol and their photoelectric conversion under near-infrared light. New Journal of Chemistry, 36(1), 97-101.

[107] Jin, S. H., Kim, D. H., Jun, G. H., Hong, S. H., \& Jeon, S. (2013). Tuning the Photoluminescence of Graphene Quantum Dots through the Charge Transfer Effect of Functional Groups. ACS Nano, 7(2), 1239-1245.

[108] Peng, J., Gao, W., Gupta, B. K., Liu, Z., Romero-Aburto, R., Ge, L., Song, L., Alemany, L. B., Zhan, X., Gao, G., Vithayathil, S. A., Kaipparettu, B. A., Marti, A. A., Hayashi, T., Zhu, J. J., \& Ajayan, P. M. (2012). Graphene quantum dots derived from carbon fibers. Nano Letters, 12(2), 844-849.

[109] Liu, F., Jang, M. H., Ha, H. D., Kim, J. H., Cho, Y. H., \& Seo, T. S. (2013). Facile synthetic method for pristine graphene quantum dots and graphene oxide quantum dots: origin of blue and green luminescence. Advanced Materials, 25(27), 3657-3662.

[110] Zhu, S. J., Zhang, J. H., Liu, X., Li, B., Wang, X. F., Tang, S. J., Meng, Q. N., Li, Y. F., Shi, C., Hu, R., \& Yang, B. (2012). Graphene quantum dots with controllable surface oxidation, tunable fluorescence and up-conversion emission. RSC Advances, 2(7), 2717-2720.

[111] Li, Y., Hu, Y., Zhao, Y., Shi, G., Deng, L., Hou, Y., \& Qu, L. (2011). An electrochemical avenue to green-luminescent graphene quantum dots as potential electron-acceptors for photovoltaics. Advanced Materials, 23(6), 776-780.

[112] Zhang, M., Bai, L., Shang, W., Xie, W., Ma, H., Fu, Y., Fang, D., Sun, H., Fan, L., Han, M., Liu, C., \& Yang, S. (2012). Facile synthesis of water-soluble, highly fluorescent graphene quantum dots as a robust biological label for stem cells. Journal of Materials Chemistry, 22(15), 7461.

[113] Lin, L. X., \& Zhang, S. W. (2012). Creating high yield water soluble luminescent graphene quantum dots via exfoliating and disintegrating carbon nanotubes and graphite flakes. Chemical Communications, 48(82), 10177-10179.

[114] Lee, J., Kim, K., Park, W. I., Kim, B. H., Park, J. H., Kim, T. H., Bong, S., Kim, C. H., Chae, G., Jun, M., Hwang, Y., Jung, Y. S., \& Jeon, S. (2012). Uniform graphene quantum dots patterned from self-assembled silica nanodots. Nano Letters, 12(12), 6078-6083.

[115] Mohanty, N., Moore, D., Xu, Z., Sreeprasad, T. S., Nagaraja, A., Rodriguez, A. A., \& Berry, V. (2012). Nanotomy-based production of transferable and dispersible graphene nanostructures of controlled shape and size. Nature Communications, 3, 844.

[116] Shomali, A., Valizadeh, H., Banan, A., \& Mohammad-Rezaei, R. (2015). Efficient synthesis of xanthene derivatives using carboxyl functionalized graphene quantum dots as an acidic nano-catalyst under microwave irradiation. RSC Advanced, 5(107), 88202-88208.

[117] Valizadeh, H., Shomali, A., Nourshargh, S., \& Mohammad-Rezaei, R. (2015). Carboxyl and nitrite functionalized graphene quantum dots as a highly active reagent and catalyst for rapid diazotization reaction and synthesis of azo-dyes under solventfree conditions. Dyes and Pigments, 113, 522-528. 
[118] Yeh, T. F., Teng, C. Y., Chen, S. J., \& Teng, H. (2014). Nitrogen-doped graphene oxide quantum dots as photocatalysts for overall water-splitting under visible light illumination. Advanced Materials, 26(20), 3297-3303.

[119] Yeh, T.-F., Chen, S.-J., \& Teng, H. (2015). Synergistic effect of oxygen and nitrogen functionalities for graphene-based quantum dots used in photocatalytic $\mathrm{H} 2$ production from water decomposition. Nano Energy, 12, 476-485.

[120] Li, Y., Zhao, Y., Cheng, H., Hu, Y., Shi, G., Dai, L., \& Qu, L. (2012). Nitrogen-doped graphene quantum dots with oxygen-rich functional groups. Journal of the American Chemical Society, 134(1), 15-18.

[121] Saidi, W. A. (2013). Oxygen Reduction Electrocatalysis Using N-Doped Graphene Quantum-Dots. The Journal of Physical Chemistry Letters, 4(23), 41604165.

[122] Favaro, M., Ferrighi, L., Fazio, G., Colazzo, L., Di Valentin, C., Durante, C., Sedona, F., Gennaro, A., Agnoli, S., \& Granozzi, G. (2015). Single and Multiple Doping in Graphene Quantum Dots: Unraveling the Origin of Selectivity in the Oxygen Reduction Reaction. ACS Catalysis, 5(1), 129-144.

[123] Hui, L., Huang, J., Chen, G., Zhu, Y., \& Yang, L. (2016). Antibacterial Property of Graphene Quantum Dots (Both Source Material and Bacterial Shape Matter). ACS Applied Materials \& Interfaces, 8(1), 20-25.

[124] Cai, A., Wang, Q., Chang, Y., \& Wang, X. (2017). Graphitic carbon nitride decorated with S,N co-doped graphene quantum dots for enhanced visible-lightdriven photocatalysis. Journal of Alloys and Compounds, 692, 183-189.

[125] Cai, Z., Li, F., Wu, P., Ji, L., Zhang, H., Cai, C., \& Gervasio, D. F. (2015). Synthesis of Nitrogen-Doped Graphene Quantum Dots at Low Temperature for Electrochemical Sensing Trinitrotoluene. Analytical Chemistry, 87(23), 11803-11811. [126] Zhang, L., Peng, D., Liang, R. P., \& Qiu, J. D. (2015). Nitrogen-Doped Graphene Quantum Dots as a New Catalyst Accelerating the Coordination Reaction between Cadmium(II) and 5,10,15,20-Tetrakis(1-methyl-4-pyridinio)porphyrin for Cadmium(II) Sensing. Analytical Chemistry, 87(21), 10894-10901.

[127] Chen, S., Chen, X., Xia, T., \& Ma, Q. (2016). A novel electrochemiluminescence sensor for the detection of nitroaniline based on the nitrogen-doped graphene quantum dots. Biosensors \& Bioelectronics, 85, 903-908.

[128] Yang, T., Cai, F., Zhang, X., \& Huang, Y. (2015). Nitrogen and sulfur codoped graphene quantum dots as a new fluorescent probe for Au3+ions in aqueous media. RSC Adv., 5(130), 107340-107347.

[129] He, Y., Sun, J., Feng, D., Chen, H., Gao, F., \& Wang, L. (2015). Graphene quantum dots: Highly active bifunctional nanoprobes for nonenzymatic photoluminescence detection of hydroquinone. Biosensors \& Bioelectronics, 74, 418422.

[130] Wu, X., Zhang, Y., Han, T., Wu, H., Guo, S., \& Zhang, J. (2014). Composite of graphene quantum dots and $\mathrm{Fe}_{3} \mathrm{O}_{4}$ nanoparticles: peroxidase activity and application in phenolic compound removal. RSC Advances, 4(7), 3299-3305.

[131] Wu, X., Guo, S., \& Zhang, J. (2015). Selective oxidation of veratryl alcohol with composites of $\mathrm{Au}$ nanoparticles and graphene quantum dots as catalysts. Chemical Communications, 51(29), 6318-6321.

[132] Wu, C., Yuan, Y., He, Q., \& Song, R. (2016). Two-step synthesis of Ag@GQD hybrid with enhanced photothermal effect and catalytic performance. Nanotechnology, 27(48), 48LT02. 
[133] Keshipour, S., \& Adak, K. (2016). Pd(0) supported on N-doped graphene quantum dot modified cellulose as an efficient catalyst for the green reduction of nitroaromatics. RSC Advanced, 6(92), 89407-89412.

[134] Zou, J.-P., Wang, L.-C., Luo, J., Nie, Y.-C., Xing, Q.-J., Luo, X.-B., Du, H.M., Luo, S.-L., \& Suib, S. L. (2016). Synthesis and efficient visible light photocatalytic $\mathrm{H} 2$ evolution of a metal-free g-C3N4/graphene quantum dots hybrid photocatalyst. Applied Catalysis B: Environmental, 193, 103-109.

[135] Yan, M., Hua, Y., Zhu, F., Sun, L., Gu, W., \& Shi, W. (2017). Constructing nitrogen doped graphene quantum dots- $\mathrm{ZnNb} 2 \mathrm{O} 6 / \mathrm{g}-\mathrm{C} 3 \mathrm{~N} 4$ catalysts for hydrogen production under visible light. Applied Catalysis B: Environmental, 206, 531-537.

[136] Yu, S., Zhong, Y. Q., Yu, B. Q., Cai, S. Y., Wu, L. Z., \& Zhou, Y. (2016). Graphene quantum dots to enhance the photocatalytic hydrogen evolution efficiency of anatase $\mathrm{TiO} 2$ with exposed $\{001\}$ facet. Physical Chemistry Chemical Physics, 18(30), 20338-20344.

[137] Min, S., Hou, J., Lei, Y., Ma, X., \& Lu, G. (2017). Facile one-step hydrothermal synthesis toward strongly coupled $\mathrm{TiO}_{2}$ /graphene quantum dots photocatalysts for efficient hydrogen evolution. Applied Surface Science, 396, 13751382.

[138] He, G., Song, Y., Liu, K., Walter, A., Chen, S., \& Chen, S. (2013). Oxygen Reduction Catalyzed by Platinum Nanoparticles Supported on Graphene Quantum Dots. ACS Catalysis, 3(5), 831-838.

[139] Wang, L., Hu, C., Zhao, Y., Hu, Y., Zhao, F., Chen, N., \& Qu, L. (2014). A dually spontaneous reduction and assembly strategy for hybrid capsules of graphene quantum dots with platinum-copper nanoparticles for enhanced oxygen reduction reaction. Carbon, 74, 170-179.

[140] Deming, C. P., Mercado, R., Gadiraju, V., Sweeney, S. W., Khan, M., \& Chen, S. (2015). Graphene Quantum Dots-Supported Palladium Nanoparticles for Efficient Electrocatalytic Reduction of Oxygen in Alkaline Media. ACS Sustainable Chemistry \& Engineering, 3(12), 3315-3323.

[141] Deming, C. P., Mercado, R., Lu, J. E., Gadiraju, V., Khan, M., \& Chen, S. (2016). Oxygen Electroreduction Catalyzed by Palladium Nanoparticles Supported on Nitrogen-Doped Graphene Quantum Dots: Impacts of Nitrogen Dopants. ACS Sustainable Chemistry \& Engineering, 4(12), 6580-6589.

[142] Liu, K., Song, Y., \& Chen, S. (2016). Oxygen reduction catalyzed by nanocomposites based on graphene quantum dots-supported copper nanoparticles. International Journal of Hydrogen Energy, 41(3), 1559-1567.

[143] Xu, C., Han, Q., Zhao, Y., Wang, L., Li, Y., \& Qu, L. (2015). Sulfur-doped graphitic carbon nitride decorated with graphene quantum dots for an efficient metalfree electrocatalyst. Journal of Materials Chemistry A, 3(5), 1841-1846.

[144] Wang, S., Cole, I. S., \& Li, Q. (2016). Quantum-confined bandgap narrowing of $\mathrm{TiO} 2$ nanoparticles by graphene quantum dots for visible-light-driven applications. Chemical Communications, 52(59), 9208-9211.

[145] Qu, D., Zheng, M., Du, P., Zhou, Y., Zhang, L., Li, D., Tan, H., Zhao, Z., Xie, Z., \& Sun, Z. (2013). Highly luminescent S, N co-doped graphene quantum dots with broad visible absorption bands for visible light photocatalysts. Nanoscale, 5(24), 12272-12277.

[146] Li, Y., Wang, L., Ge, J., Wang, J., Li, Q., Wan, W., Zhang, B., Liu, X., \& Xue, $\mathrm{W}$. (2016). Graphene quantum dots modified $\mathrm{ZnO}+\mathrm{Cu}$ heterostructure photocatalysts with enhanced photocatalytic performance. RSC Adv., 6(108), 106508106515. 
[147] Cai, A., Wang, X., Qi, Y., \& Ma, Z. (2017). Hierarchical ZnO/S,N:GQD composites: Biotemplated synthesis and enhanced visible-light-driven photocatalytic activity. Applied Surface Science, 391, 484-490.

[148] Wang, J., Li, Y., Ge, J., Zhang, B. P., \& Wan, W. (2015). Improving photocatalytic performance of $\mathrm{ZnO}$ via synergistic effects of $\mathrm{Ag}$ nanoparticles and graphene quantum dots. Physical Chemistry Chemical Physics, 17(28), 18645-18652. [149] Zhao, X., He, D., Wang, Y., Hu, Y., \& Fu, C. (2016). Au nanoparticles and graphene quantum dots co-modified glassy carbon electrode for catechol sensing. Chemical Physics Letters, 647, 165-169.

[150] Ju, J., \& Chen, W. (2015). In situ growth of surfactant-free gold nanoparticles on nitrogen-doped graphene quantum dots for electrochemical detection of hydrogen peroxide in biological environments. Analytical Chemistry, 87(3), 1903-1910.

[151] Ran, X., Sun, H., Pu, F., Ren, J., \& Qu, X. (2013). Ag nanoparticle-decorated graphene quantum dots for label-free, rapid and sensitive detection of $\mathrm{Ag}+$ and biothiols. Chemical Communications, 49(11), 1079-1081.

[152] Guo, C. X., Dong, Y., Yang, H. B., \& Li, C. M. (2013). Graphene Quantum Dots as a Green Sensitizer to Functionalize ZnO Nanowire Arrays on F-Doped SnO2Glass for Enhanced Photoelectrochemical Water Splitting. Advanced Energy Materials, 3(8), 997-1003.

[153] Yu, Y., Ren, J., \& Meng, M. (2013). Photocatalytic hydrogen evolution on graphene quantum dots anchored $\mathrm{TiO} 2$ nanotubes-array. International Journal of Hydrogen Energy, 38(28), 12266-12272.

[154] Xu, Z., Yin, M., Sun, J., Ding, G., Lu, L., Chang, P., Chen, X., \& Li, D. (2016). 3D periodic multiscale $\mathrm{TiO}(2)$ architecture: a platform decorated with graphene quantum dots for enhanced photoelectrochemical water splitting. Nanotechnology, 27(11), 115401.

[155] Sudhagar, P., Herraiz-Cardona, I., Park, H., Song, T., Noh, S. H., Gimenez, S., Sero, I. M., Fabregat-Santiago, F., Bisquert, J., Terashima, C., Paik, U., Kang, Y. S., Fujishima, A., \& Han, T. H. (2016). Exploring Graphene Quantum Dots/TiO2 interface in photoelectrochemical reactions: Solar to fuel conversion. Electrochimica Acta, 187, 249-255.

[156] Pan, D., Xi, C., Li, Z., Wang, L., Chen, Z., Lu, B., \& Wu, M. (2013). Electrophoretic fabrication of highly robust, efficient, and benign heterojunction photoelectrocatalysts based on graphene-quantum-dot sensitized $\mathrm{TiO}_{2}$ nanotube arrays. Journal of Materials Chemistry A, 1(11), 3551.

[157] Gupta, B. K., Kedawat, G., Agrawal, Y., Kumar, P., Dwivedi, J., \& Dhawan, S. K. (2015). A novel strategy to enhance ultraviolet light driven photocatalysis from graphene quantum dots infilled $\mathrm{TiO}_{2}$ nanotube arrays. RSC Advanced, 5(14), 1062310631.

[158] Ebrahimi, M., Samadi, M., Yousefzadeh, S., Soltani, M., Rahimi, A., Chou, T.-c., Chen, L.-C., Chen, K.-H., \& Moshfegh, A. Z. (2017). Improved Solar-Driven Photocatalytic Activity of Hybrid Graphene Quantum Dots/ZnO Nanowires: A Direct Z-Scheme Mechanism. ACS Sustainable Chemistry \& Engineering, 5(1), 367-375.

[159] Quan, B., Liu, W., Liu, Y., Zheng, Y., Yang, G., \& Ji, G. (2016). Quasinoble-metal graphene quantum dots deposited stannic oxide with oxygen vacancies: Synthesis and enhanced photocatalytic properties. Journal of Colloid Interface Science, 481, 13-19.

[160] Lei, Z. D., Wang, J. J., Wang, L., Yang, X. Y., Xu, G., \& Tang, L. (2016). Efficient photocatalytic degradation of ibuprofen in aqueous solution using novel 
visible-light responsive graphene quantum dot/AgVO3 nanoribbons. Journal of Hazardous Materials, 312, 298-306.

[161] Tang, L., Wang, J, Jia, C., Lv, G., Xu, G., Li, W., Wang, L., Zhang, J., \& Wu, M. (2017). Simulated solar driven catalytic degradation of psychiatric drug carbamazepine with binary BiVO4 heterostructures sensitized by graphene quantum dots. Applied Catalysis B: Environmental, 205, 587-596.

[162] Guo, J., Zhu, H., Sun, Y., Tang, L., \& Zhang, X. (2016). Doping MoS2 with Graphene Quantum Dots: Structural and Electrical Engineering towards Enhanced Electrochemical Hydrogen Evolution. Electrochimica Acta, 211, 603-610.

[163] Liu, X., Jian, X., Yang, H., Song, X., \& Liang, Z. (2016). A photocatalytic graphene quantum dots- $\mathrm{Cu} 2 \mathrm{O} /$ bipolar membrane as a separator for water splitting. New Journal of Chemistry, 40(4), 3075-3079.

[164] Wang, Q., Zhu, N., Liu, E., Zhang, C., Crittenden, J. C., Zhang, Y., \& Cong, Y. (2017). Fabrication of visible-light active $\mathrm{Fe}_{2} \mathrm{O}_{3}$-GQDs/NF-TiO ${ }_{2}$ composite film with highly enhanced photoelectrocatalytic performance. Applied Catalysis B: Environmental, 205, 347-356.

[165] Fan, M., Zhu, C., Yang, J., \& Sun, D. (2016). Facile self-assembly N-doped graphene quantum dots/graphene for oxygen reduction reaction. Electrochimica Acta, 216, 102-109.

[166] Zhang, B., Xiao, C., Xiang, Y., Dong, B., Ding, S., \& Tang, Y. (2016). Nitrogen-Doped Graphene Quantum Dots Anchored on Thermally Reduced Graphene Oxide as an Electrocatalyst for the Oxygen Reduction Reaction. ChemElectroChem, 3(6), 864-870.

[167] Fei, H. L., Ye, R. Q., Ye, G. L., Gong, Y. J., Peng, Z. W., Fan, X. J., Samuel, E. L. G., Ajayan, P. M., \& Tour, J. M. (2014). Boron- and Nitrogen-Doped Graphene Quantum Dots/Graphene Hybrid Nanoplatelets as Efficient Electrocatalysts for Oxygen Reduction. ACS Nano, 8(10), 10837-10843.

[168] Luo, Z., Yang, D., Qi, G., Shang, J., Yang, H., Wang, Y., Yuwen, L., Yu, T., Huang, W., \& Wang, L. (2014). Microwave-assisted solvothermal preparation of nitrogen and sulfur co-doped reduced graphene oxide and graphene quantum dots hybrids for highly efficient oxygen reduction. Journal of Materials Chemistry A, 2(48), 20605-20611.

[169] Jin, H., Huang, H., He, Y., Feng, X., Wang, S., Dai, L., \& Wang, J. (2015). Graphene Quantum Dots Supported by Graphene Nanoribbons with Ultrahigh Electrocatalytic Performance for Oxygen Reduction. Journal of the American Chemical Society, 137(24), 7588-7591.

[170] Roy, P., Ravindranath, R., Periasamy, A. P., Lien, C.-W., Liang, C.-T., \& Chang, H.-T. (2016). Green synthesis of Si-GQD nanocomposites as cost-effective catalysts for oxygen reduction reaction. RSC Advanced, 6(110), 108941-108947.

[171] Song, X., Zhou, Q., Zhang, T., Xu, H., \& Wang, Z. (2016). Pressure-assisted preparation of graphene oxide quantum dot-incorporated reverse osmosis membranes: antifouling and chlorine resistance potentials. Journal of Materials Chemistry A, 4(43), 16896-16905.

[172] Fardindoost, S., Iraji zad, A., Hosseini, Z. S., \& Hatamie, S. (2016). Detecting hydrogen using graphene quantum dots/WO3 thin films. Materials Research Express, 3(11), 116407.

[173] Li, Z., Ye, R., Feng, R., Kang, Y., Zhu, X., Tour, J. M., \& Fang, Z. (2015). Graphene Quantum Dots Doping of MoS2 Monolayers. Advanced Materials, 27(35), 5235-5240. 
[174] Tian, H., Shen, K., Hu, X., Qiao, L., \& Zheng, W. (2017). N, S co-doped graphene quantum dots-graphene-TiO 2 nanotubes composite with enhanced photocatalytic activity. Journal of Alloys and Compounds, 691, 369-377.

[175] Wang, C. C., \& Lu, S. Y. (2015). Carbon black-derived graphene quantum dots composited with carbon aerogel as a highly efficient and stable reduction catalyst for the iodide/tri-iodide couple. Nanoscale, 7(3), 1209-1215.

[176] Hao, Y., Dong, X., Wang, X., Zhai, S., Ma, H., \& Zhang, X. (2016). Controllable electrostatic self-assembly of sub-3 nm graphene quantum dots incorporated into mesoporous Bi2MoO6frameworks: efficient physical and chemical simultaneous co-catalysis for photocatalytic oxidation. Journal of Materials Chemistry A, 4(21), 8298-8307.

[177] Liu, J., Xu, H., Xu, Y., Song, Y., Lian, J., Zhao, Y., Wang, L., Huang, L., Ji, H., \& Li, H. (2017). Graphene quantum dots modified mesoporous graphite carbon nitride with significant enhancement of photocatalytic activity. Applied Catalysis B: Environmental, 207, 429-437.

[178] Guo, B., Yu, K., Li, H., Qi, R., Zhang, Y., Song, H., Tang, Z., Zhu, Z., \& Chen, M. (2017). Coral-Shaped MoS2 Decorated with Graphene Quantum Dots Performing as a Highly Active Electrocatalyst for Hydrogen Evolution Reaction. ACS Applied Materials \& Interfaces, 9(4), 3653-3660.

[179] Vinoth, R., Patil, I. M., Pandikumar, A., Kakade, B. A., Huang, N. M., Dionysios, D. D., \& Neppolian, B. (2016). Synergistically Enhanced Electrocatalytic Performance of an N-Doped Graphene Quantum Dot-Decorated 3D MoS2-Graphene Nanohybrid for Oxygen Reduction Reaction. ACS Omega, 1(5), 971-980.

[180] Tolga Çolak, A., Eren, T., Yola, M. L., Beşli, E., Şahin, O., \& Atar, N. (2016). 3D Polyoxometalate-Functionalized Graphene Quantum Dots with Mono-Metallic and Bi-Metallic Nanoparticles for Application in Direct Methanol Fuel Cells. Journal of The Electrochemical Society, 163(10), F1237-F1244.

[181] Pei, H. M., Zhu, S. Y., Yang, M. H., Kong, R. M., Zheng, Y. Q., \& Qu, F. L. (2015). Graphene oxide quantum dots@silver core-shell nanocrystals as turn-on fluorescent nanoprobe for ultrasensitive detection of prostate specific antigen. Biosensors \& Bioelectronics, 74, 909-914.

[182] Xu, X. H., \& Wang, Y. H. (2015). A Novel Sensor for Sensitive and Selective Detection of Iodide Using Turn-on Fluorescence Graphene Quantum Dots/Ag Nanocomposite. Analytical Sciences, 31(8), 787-791.

[183] Xiao, F. X., Pagliaro, M., Xu, Y. J., \& Liu, B. (2016). Layer-by-layer assembly of versatile nanoarchitectures with diverse dimensionality: a new perspective for rational construction of multilayer assemblies. Chemical Society Reviews, 45(11), 3088-3121.

[184] Ahn, E., Lee, T., Gu, M., Park, M., Min, S. H., \& Kim, B. S. (2017). Layerby-Layer Assembly for Graphene-Based Multilayer Nanocomposites: The Field Manual. Chemistry of Materials, 29(1), 69-79.

[185] Zeng, Z. P., Yu, D. S., He, Z. M., Liu, J., Xiao, F. X., Zhang, Y., Wang, R., Bhattacharyya, D., \& Tan, T. T. Y. (2016). Graphene Oxide Quantum Dots Covalently Functionalized PVDF Membrane with Significantly-Enhanced Bactericidal and Antibiofouling Performances. Scientific Reports, 6.

[186] Hong, J., Shah, N. J., Drake, A. C., DeMuth, P. C., Lee, J. B., Chen, J. Z., \& Hammond, P. T. (2012). Graphene Multilayers as Gates for Multi-Week Sequential Release of Proteins from Surfaces. ACS Nano, 6(1), 81-88. 
[187] Dotzauer, D. M., Dai, J. H., Sun, L., \& Bruening, M. L. (2006). Catalytic membranes prepared using layer-by-layer adsorption of polyelectrolyte/metal nanoparticle films in porous supports. Nano Lettersers, 6(10), 2268-2272.

[188] Yang, J., Lee, J. Y., \& Too, H. P. (2005). Core-shell Ag-Au nanoparticles from replacement reaction in organic medium. Journal of Physical Chemistry B, 109(41), 19208-19212.

[189] Lin, S. Y., Wu, S. H., \& Chen, C. H. (2006). A simple strategy for prompt visual sensing by gold nanoparticles: General applications of interparticle hydrogen bonds. Angewandte Chemie-International Edition, 45(30), 4948-4951.

[190] Li, D., Muller, M. B., Gilje, S., Kaner, R. B., \& Wallace, G. G. (2008). Processable aqueous dispersions of graphene nanosheets. Nature Nanotechnology, 3(2), 101-105.

[191] Yuan, W. Y., \& Li, C. M. (2009). Direct Modulation of Localized Surface Plasmon Coupling of Au Nanoparticles on Solid Substrates via Weak PolyelectrolyteMediated Layer-by-Layer Self Assembly. Langmuir, 25(13), 7578-7585.

[192] Xiao, F. X. (2012). Layer-by-Layer Self-Assembly Construction of Highly Ordered Metal-TiO2 Nanotube Arrays Heterostructures (M/TNTs, M = Au, Ag, Pt) with Tunable Catalytic Activities. Journal of Physical Chemistry C, 116(31), 1648716498.

[193] Luo, J., Njoki, P. N., Lin, Y., Mott, D., Wang, L. Y., \& Zhong, C. J. (2006). Characterization of carbon-supported AuPt nanoparticles for electrocatalytic methanol oxidation reaction. Langmuir, 22(6), 2892-2898.

[194] Li, M., Cullen, D. A., Sasaki, K., Marinkovic, N. S., More, K., \& Adzic, R. R. (2013). Ternary Electrocatalysts for Oxidizing Ethanol to Carbon Dioxide: Making Ir Capable of Splitting C-C Bond. Journal of the American Chemical Society, 135(1), 132-141.

[195] Kowal, A., Li, M., Shao, M., Sasaki, K., Vukmirovic, M. B., Zhang, J., Marinkovic, N. S., Liu, P., Frenkel, A. I., \& Adzic, R. R. (2009). Ternary Pt/Rh/SnO2 electrocatalysts for oxidizing ethanol to CO2. Nature Materials, 8(4), 325-330.

[196] Yan, S. H., Zhang, S. C., Lin, Y., \& Liu, G. R. (2011). Electrocatalytic Performance of Gold Nanoparticles Supported on Activated Carbon for Methanol Oxidation in Alkaline Solution. Journal of Physical Chemistry C, 115(14), 6986-6993. [197] Lee, E. P., Peng, Z. M., Chen, W., Chen, S. W., Yang, H., \& Xia, Y. N. (2008). Electrocatalytic Properties of Pt Nanowires Supported on Pt and W Gauzes. ACS Nano, 2(10), 2167-2173.

[198] Chang, B. Y., \& Park, S. M. (2010). Electrochemical Impedance Spectroscopy. Annual Review of Analytical Chemistry, Vol 3, 3, 207-229.

[199] Natan, M. J., Thackeray, J. W., \& Wrighton, M. S. (1986). Interaction of thiols with n-type cadmium sulfide and n-type cadmium selenide in aqueous solutions: adsorption of thiolate anion and efficient photoelectrochemical oxidation to disulfides. The Journal of Physical Chemistry, 90(17), 4089-4098.

[200] Lin, Y. G., Hsu, Y. K., Chen, Y. C., Wang, S. B., Miller, J. T., Chen, L. C., \& Chen, K. H. (2012). Plasmonic Ag@Ag-3(PO4)(1-x) nanoparticle photosensitized $\mathrm{ZnO}$ nanorod-array photoanodes for water oxidation. Energy \& Environmental Science, 5(10), 8917-8922.

[201] Chen, H. M., Chen, C. K., Tseng, M. L., Wu, P. C., Chang, C. M., Cheng, L. C., Huang, H. W., Chan, T. S., Huang, D. W., Liu, R. S., \& Tsai, D. P. (2013). Plasmonic $\mathrm{ZnO} / \mathrm{Ag}$ Embedded Structures as Collecting Layers for Photogenerating Electrons in Solar Hydrogen Generation Photoelectrodes. Small, 9(17), 2926-2936. 
[202] Thiyagarajan, P., Ahn, H. J., Lee, J. S., Yoon, J. C., \& Jang, J. H. (2013). Hierarchical Metal/Semiconductor Nanostructure for Efficient Water Splitting. Small, 9(13), 2341-2347.

[203] Subramanian, V., Wolf, E. E., \& Kamat, P. V. (2003). Green emission to probe photoinduced charging events in $\mathrm{ZnO}-\mathrm{Au}$ nanoparticles. Charge distribution and fermi-level equilibration. Journal of Physical Chemistry B, 107(30), 7479-7485. [204] Lin, Y. G., Hsu, Y. K., Chen, Y. C., Chen, L. C., Chen, S. Y., \& Chen, K. H. (2012). Visible-light-driven photocatalytic carbon-doped porous $\mathrm{ZnO}$ nanoarchitectures for solar water-splitting. Nanoscale, 4(20), 6515-6519.

[205] Xie, S. L., Lu, X. H., Zhai, T., Li, W., Yu, M. H., Liang, C. L., \& Tong, Y. X. (2012). Enhanced photoactivity and stability of carbon and nitrogen co-treated $\mathrm{ZnO}$ nanorod arrays for photoelectrochemical water splitting. Journal of Materials Chemistry, 22(28), 14272-14275.

[206] Yang, X. Y., Wolcott, A., Wang, G. M., Sobo, A., Fitzmorris, R. C., Qian, F., Zhang, J. Z., \& Li, Y. (2009). Nitrogen-Doped ZnO Nanowire Arrays for Photoelectrochemical Water Splitting. Nano Lettersers, 9(6), 2331-2336.

[207] Li, H. X., Cheng, C. W., Li, X. L., Liu, J. P., Guan, C., Tay, Y. Y., \& Fan, H. J. (2012). Composition-Graded ZnxCd1-xSe@ZnO Core-Shell Nanowire Array Electrodes for Photoelectrochemical Hydrogen Generation. Journal of Physical Chemistry C, 116(5), 3802-3807.

[208] Wang, G. M., Yang, X. Y., Qian, F., Zhang, J. Z., \& Li, Y. (2010). DoubleSided CdS and CdSe Quantum Dot Co-Sensitized ZnO Nanowire Arrays for Photoelectrochemical Hydrogen Generation. Nano Lettersers, 10(3), 1088-1092.

[209] Yin, Z. Y., Wang, Z., Du, Y. P., Qi, X. Y., Huang, Y. Z., Xue, C., \& Zhang, H. (2012). Full Solution-Processed Synthesis of All Metal Oxide-Based Tree-like Heterostructures on Fluorine-Doped Tin Oxide for Water Splitting. Advanced Materials, 24(39), 5374-5378.

[210] Luna, S. M., Silva, S. S., Gomes, M. E., Mano, J. F., \& Reis, R. L. (2011). Cell Adhesion and Proliferation onto Chitosan-based Membranes Treated by Plasma Surface Modification. Journal of Biomaterials Applications, 26(1), 101-116.

[211] Guo, C. X., Dong, Y. Q., Yang, H. B., \& Li, C. M. (2013). Graphene Quantum Dots as a Green Sensitizer to Functionalize ZnO Nanowire Arrays on FDoped SnO2 Glass for Enhanced Photoelectrochemical Water Splitting. Advanced Energy Materials, 3(8), 997-1003.

[212] Xue, Y. H., Liu, J., Chen, H., Wang, R. G., Li, D. Q., Qu, J., \& Dai, L. M. (2012). Nitrogen-Doped Graphene Foams as Metal-Free Counter Electrodes in HighPerformance Dye-Sensitized Solar Cells. Angewandte Chemie-International Edition, 51(48), 12124-12127.

[213] Susi, T., Kotakoski, J., Arenal, R., Kurasch, S., Jiang, H., Skakalova, V., Stephan, O., Krasheninnikov, A. V., Kauppinen, E. I., Kaiser, U., \& Meyer, J. C. (2012). Atomistic Description of Electron Beam Damage in Nitrogen-Doped Graphene and Single-Walled Carbon Nanotubes. ACS Nano, 6(10), 8837-8846.

[214] Mattevi, C., Eda, G., Agnoli, S., Miller, S., Mkhoyan, K. A., Celik, O., Mastrogiovanni, D., Granozzi, G., Garfunkel, E., \& Chhowalla, M. (2009). Evolution of Electrical, Chemical, and Structural Properties of Transparent and Conducting Chemically Derived Graphene Thin Films. Advanced Functional Materials, 19(16), 2577-2583.

[215] Zhou, X. S., Wan, L. J., \& Guo, Y. G. (2013). Binding SnO2 Nanocrystals in Nitrogen-Doped Graphene Sheets as Anode Materials for Lithium-Ion Batteries. Advanced Materials, 25(15), 2152-2157. 
[216] Yu, D. S., Nagelli, E., Du, F., \& Dai, L. M. (2010). Metal-Free Carbon Nanomaterials Become More Active than Metal Catalysts and Last Longer. Journal of Physical Chemistry Letters, 1(14), 2165-2173.

[217] Qu, D., Zheng, M., Zhang, L. G., Zhao, H. F., Xie, Z. G., Jing, X. B., Haddad, R. E., Fan, H. Y., \& Sun, Z. C. (2014). Formation mechanism and optimization of highly luminescent N-doped graphene quantum dots. Scientific Reports, 4.

[218] Sun, H. Q., Wang, Y. X., Liu, S. Z., Ge, L., Wang, L., Zhu, Z. H., \& Wang, S. B. (2013). Facile synthesis of nitrogen doped reduced graphene oxide as a superior metal-free catalyst for oxidation. Chemical Communications, 49(85), 9914-9916.

[219] Huang, M. H., Mao, S., Feick, H., Yan, H. Q., Wu, Y. Y., Kind, H., Weber, E., Russo, R., \& Yang, P. D. (2001). Room-temperature ultraviolet nanowire nanolasers. Science, 292(5523), 1897-1899.

[220] Fu, F. Y., Li, L. Y., Liu, L. J., Cai, J., Zhang, Y. P., Zhou, J. P., \& Zhang, L. N. (2015). Construction of Cellulose Based ZnO Nanocomposite Films with Antibacterial Properties through One-Step Coagulation. ACS Applied Materials \& Interfaces, 7(4), 2597-2606.

[221] Mauter, M. S., Wang, Y., Okemgbo, K. C., Osuji, C. O., Giannelis, E. P., \& Elimelech, M. (2011). Antifouling Ultrafiltration Membranes via Post-Fabrication Grafting of Biocidal Nanomaterials. ACS Applied Materials \& Interfaces, 3(8), 28612868 .

[222] Ben-Sasson, M., Zodrow, K. R., Qi, G. G., Kang, Y., Giannelis, E. P., \& Elimelech, M. (2014). Surface Functionalization of Thin-Film Composite Membranes with Copper Nanoparticles for Antimicrobial Surface Properties. Environmental Science \& Technology, 48(1), 384-393.

[223] Kang, S., Pinault, M., Pfefferle, L. D., \& Elimelech, M. (2007). Single-walled carbon nanotubes exhibit strong antimicrobial activity. Langmuir, 23(17), 8670-8673. [224] Tiraferri, A., Vecitis, C. D., \& Elimelech, M. (2011). Covalent Binding of Single-Walled Carbon Nanotubes to Polyamide Membranes for Antimicrobial Surface Properties. ACS Applied Materials \& Interfaces, 3(8), 2869-2877.

[225] Liu, S. B., Zeng, T. H., Hofmann, M., Burcombe, E., Wei, J., Jiang, R. R., Kong, J., \& Chen, Y. (2011). Antibacterial Activity of Graphite, Graphite Oxide, Graphene Oxide, and Reduced Graphene Oxide: Membrane and Oxidative Stress. ACS Nano, 5(9), 6971-6980.

[226] Perreault, F., Tousley, M. E., \& Elimelech, M. (2014). Thin-Film Composite Polyamide Membranes Functionalized with Biocidal Graphene Oxide Nanosheets. Environmental Science \& Technology Letters, 1(1), 71-76.

[227] Shen, J. H., Zhu, Y. H., Yang, X. L., \& Li, C. Z. (2012). Graphene quantum dots: emergent nanolights for bioimaging, sensors, catalysis and photovoltaic devices. Chemical Communications, 48(31), 3686-3699.

[228] Wu, X., Tian, F., Wang, W. X., Chen, J., Wu, M., \& Zhao, J. X. (2013). Fabrication of highly fluorescent graphene quantum dots using L-glutamic acid for in vitro/in vivo imaging and sensing. Journal of Materials Chemistry C, 1(31), 46764684.

[229] Zheng, A. X., Cong, Z. X., Wang, J. R., Li, J., Yang, H. H., \& Chen, G. N. (2013). Highly-efficient peroxidase-like catalytic activity of graphene dots for biosensing. Biosensors \& Bioelectronics, 49, 519-524.

[230] Nurunnabi, M., Khatun, Z., Huh, K. M., Park, S. Y., Lee, D. Y., Cho, K. J., \& Lee, Y. K. (2013). In Vivo Biodistribution and Toxicology of Carboxylated Graphene Quantum Dots. ACS Nano, 7(8), 6858-6867. 
[231] Wu, C. Y., Wang, C., Han, T., Zhou, X. J., Guo, S. W., \& Zhang, J. Y. (2013). Insight into the Cellular Internalization and Cytotoxicity of Graphene Quantum Dots. Advanced Healthcare Materials, 2(12), 1613-1619.

[232] Cong, H. P., Chen, J. F., \& Yu, S. H. (2014). Graphene-based macroscopic assemblies and architectures: an emerging material system. Chemical Society Reviews, 43(21), 7295-7325.

[233] Yu, L., Zhang, Y. T., Zhang, B., Liu, J. D., Zhang, H. Q., \& Song, C. H. (2013). Preparation and characterization of HPEI-GO/PES ultrafiltration membrane with antifouling and antibacterial properties. Journal of Membrane Science, 447, 452462.

[234] Wick, P., Louw-Gaume, A. E., Kucki, M., Krug, H. F., Kostarelos, K., Fadeel, B., Dawson, K. A., Salvati, A., Vazquez, E., Ballerini, L., Tretiach, M., Benfenati, F., Flahaut, E., Gauthier, L., Prato, M., \& Bianco, A. (2014). Classification Framework for Graphene-Based Materials. Angewandte Chemie-International Edition, 53(30), 7714-7718.

[235] Zuo, G. Z., \& Wang, R. (2013). Novel membrane surface modification to enhance anti-oil fouling property for membrane distillation application. Journal of Membrane Science, 447, 26-35.

[236] Wang, P., Tan, K. L., Kang, E. T., \& Neoh, K. G. (2002). Plasma-induced immobilization of poly(ethylene glycol) onto poly(vinylidene fluoride) microporous membrane. Journal of Membrane Science, 195(1), 103-114.

[237] Ruiz, O. N., Fernando, K. A. S., Wang, B. J., Brown, N. A., Luo, P. G., McNamara, N. D., Vangsness, M., Sun, Y. P., \& Bunker, C. E. (2011). Graphene Oxide: A Nonspecific Enhancer of Cellular Growth. ACS Nano, 5(10), 8100-8107.

[238] Akhavan, O., \& Ghaderi, E. (2010). Toxicity of Graphene and Graphene Oxide Nanowalls Against Bacteria. ACS Nano, 4(10), 5731-5736.

[239] Angert, E. R. (2005). Alternatives to binary fission in bacteria. Nature Reviews Microbiology, 3(3), 214-224.

[240] Tu, Y. S., Lv, M., Xiu, P., Huynh, T., Zhang, M., Castelli, M., Liu, Z. R., Huang, Q., Fan, C. H., Fang, H. P., \& Zhou, R. H. (2013). Destructive extraction of phospholipids from Escherichia coli membranes by graphene nanosheets. Nature Nanotechnology, 8(8), 594-601.

[241] Li, Y., \& Shimizu, H. (2008). Conductive PVDF/PA6/CNTs nanocomposites fabricated by dual formation of cocontinuous and nanodispersion structures. Macromolecules, 41(14), 5339-5344.

[242] Musico, Y. L. F., Santos, C. M., Dalida, M. L. P., \& Rodrigues, D. F. (2014). Surface Modification of Membrane Filters Using Graphene and Graphene OxideBased Nanomaterials for Bacterial Inactivation and Removal. ACS Sustainable Chemistry \& Engineering, 2(7), 1559-1565.

[243] Liu, F., Jang, M. H., Ha, H. D., Kim, J. H., Cho, Y. H., \& Seo, T. S. (2013). Facile Synthetic Method for Pristine Graphene Quantum Dots and Graphene Oxide Quantum Dots: Origin of Blue and Green Luminescence. Advanced Materials, 25(27), 3657-3662. 